
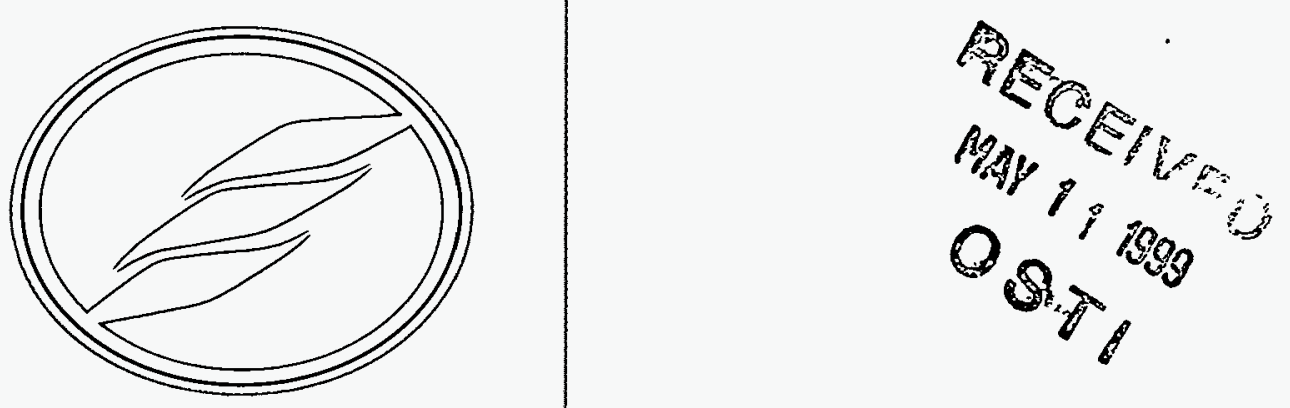

INEEL/EXT-98-01166

November 1998

\title{
WERF MACT Feasibility Study Report
}

\section{WERF MACT Feasibility Study Team}

LOCKHEED MARTIN 


3
3
3
3
3
3
3
3
3
3
3
3
3
3
3
3
3
3
3
3
3
3
3
3
3
3
3
3
3
3
3
3
3
3
3
3
3
3




\title{
WERF MACT Feasibility Study Report
}

\author{
WERF MACT Feasibility Study Team
}

Published November 1998

\section{Idaho National Engineering and Environmental Laboratory \\ Lockheed Martin Idaho Technologies Company Idaho Falls, Idaho 83415}

Prepared for the

U.S. Department of Energy

Assistant Secretary for Environmental Management

Under DOE Idaho Operations Office

Contract DE-AC07-94ID13223 


\section{ABSTRACT}

This study was undertaken to determine the technical feasibility of upgrading the Waste Experimental Reduction Facility (WERF) to meet the offgas emission limits proposed in the Maximum Achievable Control Technologies rule. Four practicable offgas treatment processes were identified, which, if installed, would enable the WERF to meet the anticipated MACT emission limits for dioxins and furans (D/F), hydrochloric acid $(\mathrm{HCl})$, and mercury $(\mathrm{Hg})$. Due to the three-year time restraint for MACT compliance, any technology chosen for the upgrade must be performed within the general plant project funding limit of $\$ 5 \mathrm{M}$.

The option selected consists of a partial-quench evaporative cooler with dry sorbent injection for $\mathrm{HCl}$ removal followed by a sulfur-impregnated activated carbon bed for $\mathrm{Hg}$ control. The planning cost estimate for implementing the option is $\$ 4.17 \mathrm{M}$ (with $24 \%$ contingency). The total estimated cost includes capital costs, design and construction costs, and project management costs. Capital costs include the purchase of a new offgas evaporative cooler, a dry sorbent injection system with reagent storage, a new fabric filter baghouse, a fixed carbon bed adsorber, and two offgas induced draft exhaust fans. It is estimated that 21 months will be required to complete the recommended modification to the WERF.

The partial-quench cooler is designed to rapidly cool the offgas exiting the secondary combustion chamber to minimize $\mathrm{D} / \mathrm{F}$ formation. Dry sorbent injection of an alkali reagent into the offgas is recommended. The alkali reacts with the $\mathrm{HCl}$ to form a salt, which is captured with the fly ash in the baghouse. A design $\mathrm{HCl}$ removal efficiency of $97.2 \%$ allows for the feeding $20 \mathrm{lbs} / \mathrm{hr}$ of chlorine to the WERF incinerator. The sorbent feed rate can be adjusted to achieve the desired $\mathrm{HCl}$ removal efficiency. A fixed bed of sulfur-impregnated carbon was conservatively sized for a total $\mathrm{Hg}$ removal capacity when feeding $10 \mathrm{~g} / \mathrm{hr} \mathrm{Hg}$ to the WERF incinerator. An added benefit for using carbon adsorption is that the activated carbon will also capture a large fraction of any residual D/F present in the offgas. 
$\therefore$ 


\section{EXECUTIVE SUMMARY}

The Waste Experimental Reduction Facility (WERF) currently incinerates low-level waste (LLW) and mixed low-level waste (MLLW). New emission standards reflecting the performance of maximum achievable control technologies (MACT) have been proposed to afford increased protection to human health and the environment. This study was undertaken to determine the technical feasibility of upgrading the WERF to meet the offgas emission limits proposed in the MACT rule. Additional objectives of this study include: (1) ensuring that a market exists for MLLW treatment at the WERF, and (2) the possibility for broadening the WERF waste acceptance criteria.

Four practical offgas treatment options were identified, which, if installed, would enable the WERF to meet the anticipated MACT emission limits for dioxins and furans (D/F), hydrochloric acid ( $\mathrm{HCl})$, and mercury $(\mathrm{Hg})$. Each option was assessed in terms of technical feasibility, relative cost, physical requirements, and the impact on the WERF waste acceptance criteria. A computer software program was used to organize the air pollution control system selection process and document the results.

If the WERF incinerator is to continue operation, the facility must be MACT compliant within three years of the date the final ruling is issued. The MACT final rule is expected to be published in April 1999. This three-year period includes the time necessary for performance testing and certification of compliance. Upgrade options costing more than $\$ 5 \mathrm{M}$ were not considered further because their funding would require congressional approval and would probably take longer than the allowable three-year time frame. The preferred air pollution control system alternative can be implemented for a total estimated cost of $\$ 4.17 \mathrm{M}$ while meeting the technical objectives for the project. The total estimated cost includes capital costs, design and construction costs, and project management costs. The cost estimate includes a $24 \%$ contingency because it is based upon preliminary information.

A WERF facility analysis revealed no significant structural or mechanical problems which would limit the continued operation of the WERF incinerator or preclude the selected MACT upgrade.

The chosen option consists of a partial-quench evaporative cooler with dry sorbent injection for $\mathrm{HCl}$ removal followed by sulfur-impregnated activated carbon bed for $\mathrm{Hg}$ control. The partial-quench cooler is designed to rapidly cool the gas exiting the secondary combustion chamber offgas to minimize D/F formation. Dry sorbent injection of an alkali reagent into the offgas is recommended for $\mathrm{HCl}$ control. The alkali reacts with the $\mathrm{HCl}$ to form a salt which is captured with the fly ash in the baghouse. A design $\mathrm{HCl}$ removal efficiency of $97.2 \%$ allows for the feeding $20 \mathrm{lbs} / \mathrm{hr}$ of chlorine to the WERF incinerator. The sorbent feed rate can be adjusted to achieve the desired removal efficiency. A fixed bed of sulfur-impregnated carbon was conservatively sized for a total $\mathrm{Hg}$ removal capacity when feeding $10 \mathrm{~g} / \mathrm{hr} \mathrm{Hg}$ to the WERF incinerator. An added benefit for using carbon adsorption is that the activated carbon will also capture a large fraction of any residual D/F present in the offgas. 
A review of a number of mixed waste databases shows that approximately $5,000 \mathrm{~m}^{3}$ of waste is available for WERF treatment in the next 5 years. Using a conservative extrapolation to the year 2010, it appears that an additional $5,000 \mathrm{~m}^{3}$ would be available for WERF. This figure does not include the significant waste volume from the other DOE incinerator sites if they are not upgraded to meet the proposed MACT rule. Depending upon the mixed waste feed, the upgraded WERF capacity ranges from $700 \mathrm{~m}^{3} / \mathrm{yr}$ to $1,300 \mathrm{~m}^{3} / \mathrm{yr}$. Based upon these aforementioned values, there is enough mixed waste within the U.S. Department of Energy (DOE) system that has been approved for WERF incineration to operate WERF at capacity for at least 10 years. This waste inventory is independent of the MACT compliance status for the other DOE mixed waste incinerators.

Since the proposed enhancement to the WERF air pollution control system removes offgas pollutants more efficiently than the current process, the WERF waste acceptance criteria can be expanded to allow more waste with higher contaminant concentrations to be treated. Additionally, if a minimum gas residence time of 2 seconds in the secondary combustion chamber of the incinerator can be maintained, polychlorinated biphenyls (PCBs) can be treated in the WERF incinerator. Since PCBs are regulated under the Toxic Substance Control Act, a Toxic Substances Control Act permit would be required before PCB incineration at WERF could occur. Preliminary calculations indicate that the gas volumetric flowrate can be operated in a range to achieve the minimum gas residence time in the secondary combustion chamber required by the Toxic Substances Control Act. 


\section{ACKNOWLEDGMENTS}

The authors of this report include Bruce Bonnema, Kevin Kooda, Kevin Liekhus, Dave Moser, and Jeff Riedesel of Lockheed Martin Idaho Technologies Company and Steve Poling and Kelly Rebish of Science Applications International Corporation. The authors wish to thank all of the people who contributed to this report: Curtis Branter, Andrea Chambers, Dennis Conley, Shannon Corrigan, Bob Shilkett, and Nick Soelberg for their technical input; Robert Bloom of Waste Management Federal Services of Hanford, Inc. and Mitchell Hahn of Waste Management, Inc. for their technical review; Susan Marushia for coordinating the report; Brian Searle for providing the drawings; Mitzi Silversmith for text processing; and Gwenna Hill for editing and preparing the report. 


\section{CONTENTS}

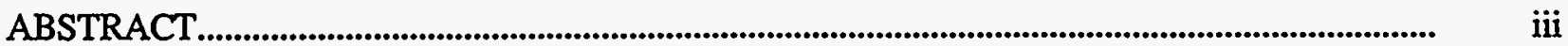

EXECUTIVE SUMMARY ................................................................................................. v

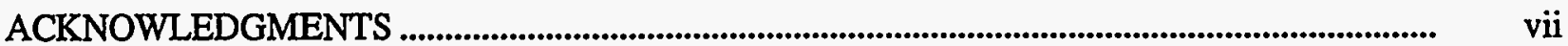

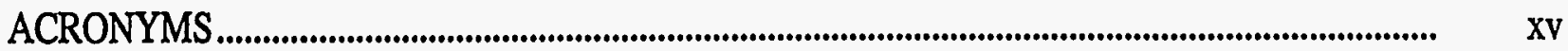

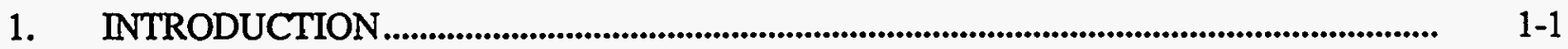

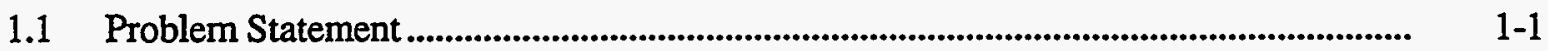

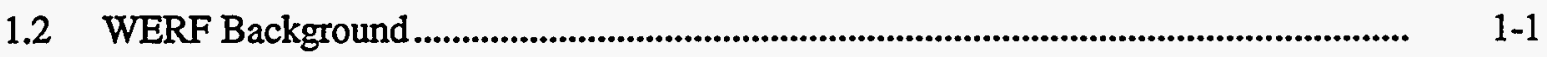

1.2.1 Incineration System Description................................................................. 1-2

1.2.2 Incineration Campaign Schedule ......................................................................... 1-4

1.3 Regulatory Background and Requirements ...................................................... 1-4

1.3.1 Proposed Rule ................................................................................................... $1-4$

1.3.2 Notice of Data Availability ..................................................................................... 1-5

1.3.3 MACT Proposed Emission Limits (May 2, 1997) .......................................... 1-6

1.3.4 MACT Emission Controls ........................................................................... 1-6

1.3.5 Continuous Emissions Monitoring.............................................................. 1-7

1.3.6 Final Rule.........................................................................................

2. MARKET ANALYSIS FOR MIXED LOW-LEVEL WASTE INCINERATION AT

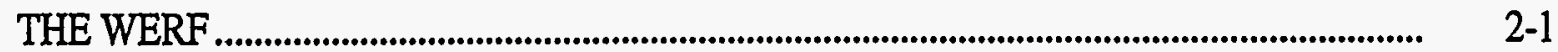

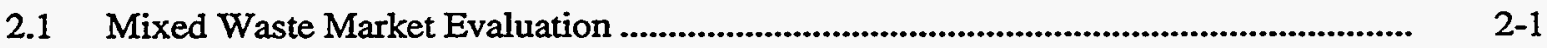

2.1.1 Mixed Waste Inventory Report-1995 ............................................................ 2-1

2.1.2 INEEL Site Treatment Plan ......................................................................... 2-1

2.1.3 Environmental Management Integration ............................................................ 2-2

2.1.4 Mixed Waste Focus Area............................................................................ 2-2

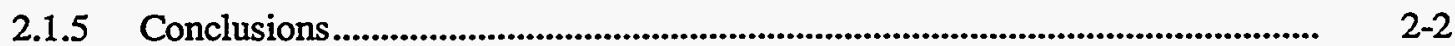

2.2 DOE Incinerator Features and MACT Compliance Status ......................................... $\quad$ 2-3

2.2.1 Consolidated Incinerator Facility ..................................................................... 2-6

2.2.2 Toxic Substances Control Act Incinerator ........................................................... 2-7

2.2.3 Waste Experimental Reduction Facility ......................................................... 2-7

2.3 Current WERF Waste Acceptance Criteria......................................................... 2-8

2.3.1 MACT Emission Limits Versus WERF's Current Emission Levels ............... . 2-10

2.4 Feasibility of Upgrading WERF to Treat PCBs......................................................... 2-12

2.4.1 Environmental Compliance Issues.................................................................. 2-.. 2-12 


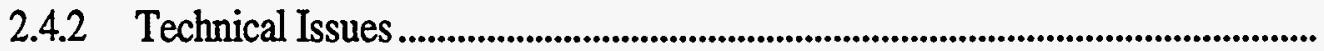

2.4.3 Other Considerations ....................................................................................

2.4.4 Recommendations.

3. EQUIPMENT STATUS FOR THE WERF INCINERATION PROCESS................................

3.1 Process Description............................................................................................................ 3-1

3.1.1 Incinerator .......................................................................................................

3.1.2 Air Pollution Control System............................................................................... 3-.

3.1.3 Continuous Emissions Monitors .......................................................................... $3-5$

3.2 Equipment Assessment ................................................................................................

3.2.1 Incinerator (Primary and Secondary Chambers) ............................................... 3-5

3.2.2 Bottom Ash Handling System............................................................................

3.2.3 Heat Exchanger .............................................................................................. $3-7$

3.2.4 Baghouse Dust Collectors.............................................................................. 3-7

3.2.5 Dust Transfer System................................................................................ 3-8

3.2.6 Main Off gas HEPA Filter Housing................................................................ 3-. 3

3.2.7 Air Pollution Control System Duct-Work, Fans, and Stack .............................. 3 3-9

3.2.8 Oxygen, Carbon Monoxide, and Hydrogenchloride Analyzers......................... 3 3-9

3.3 Conclusions and Recommendations ........................................................................ $\quad 3-10$

4. SCREENING COMMERCIALLY AVAILABLE AIR POLLUTION CONTROL TECHNOLOGIES

4.1 Hydrochloride Control Technologies.......................................................................... 4-

4.1.1 Wet Scrubber Technology .......................................................................... 4- 41

4.1.2 Semi-dry Scrubber Technology or Spray Dry Absorber..................................... $\quad 4-2$

4.1.3 Dry Scrubber Technology ....................................................................................... 4

4.2 Mercury Control Technologies ......................................................................................... 4-3

4.2.1 Particulate Matter Control Device ....................................................................... 4 4

4.2.2 Wet Scrubbing ..............................................................................................

4.2.3 Carbon Adsorption....................................................................................... 4...

4.2.4 Gold Amalgamation Filter.................................................................................

4.3 Dioxin/Furan Control Strategies .................................................................................

4.3.1 Minimize Formation ................................................................................

4.3.2 Removal .............................................................................................. 4

4.4 Other Technologies....................................................................................................... 4-.

4.5 Continuous Emission Monitor System Technologies ......................................................... 4-8

4.5.1 Particulate Matter ........................................................................................... 48 
4.5.2 Total Hydrocarbons ........................................................................................... 4.

4.5.3 Carbon Monoxide .......................................................................................... 49

5. Air Pollution Control Systems Considered for Upgrading WERF to Meet MACT................. 5-1

5.1 Dry Sorbent Injection Process Description ........................................................................ 5-1

5.1.1 Evaporative Cooler ........................................................................................... 5-3

5.1.2 Dry Sorbent Injection............................................................................................ $5-1$

5.1.3 Baghouse/Dust Transfer..................................................................................... 5-6

5.1.4 HEPA Filtration .....................................................................................................

5.1.5 Carbon Adsorption............................................................................................ 5-. 5

5.1.6 Induced Draft Fans, Emergency Power, and Stack............................................ 5-8

5.1.7 Continuous Emissions Monitors ............................................................................ 5-8

5.2 Spray Dry Absorber (Option 2) .......................................................................................

5.2.1 Spray Dryer Absorber ............................................................................................... 5-9

5.3 Wet Scrubber Downstream of Particulate Filtration (Option 3) ...................................... 5-10

5.3.1 Packed Bed Scrubber ...................................................................................... 5-10

5.3.2 Gas Reheater ........................................................................................ 5

5.4 Wet Scrubber Upstream of Particulate Removal (Option 4)......................................... $\quad 5-11$

5.4.1 Full-Quench Evaporative Cooler ........................................................................ 5-11

5.4.2 Venturi Wet Scrubber .................................................................................. $5-11$

5.4.3 Wet Electrostatic Precipitator ......................................................................... $5-11$

5.4.4 Evaporator....................................................................................................... 5

5.4.5 Condenser ...................................................................................................... 5

5.5 Selection of Recommended Air Pollution Control System............................................. 5-12

5.5.1 Technical Feasibility ................................................................................. $5-13$

5.5.2 Physical Requirements.......................................................................... 5-13

5.5.3 Impact on the WERF Waste Acceptance Criteria............................................. 5-13

5.5.4 Relative Cost.............................................................................................. $5-13$

5.5.5 Decision Plus Results................................................................................... $\quad 5-15$

5.5.6 Technical Background for Option 1.................................................................. 5-.

6. PLANNING COST ESTIMATE FOR RECOMMENDED AIR POLLUTION CONTROL SYSTEM OPTION ................................................................................................................

6.1 Capital Cost................................................................................................................... 6.

6.2 Design and Construction Costs ..................................................................................... 6-1

6.3 Management Costs.......................................................................................................... 6-7

6.4 WERF Operational Cost Evaluation for MACT Upgrades............................................ 6-7 
6.4.1 Incineration Costs Before Upgrades ................................................................. 6-7

6.4.2 Incineration Costs After Upgrades.................................................................. 6-8

6.5 Return on Investment ..........................................................................................

6.5.1 Case One-_Upgrading WERF .................................................................... 6-8

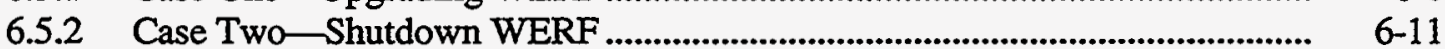

6.5.3 Conclusions.............................................................................................. $6-11$

7. PROJECT SCHEDULE .............................................................................................

7.1 Regulatory Considerations ....................................................................................... 7-1

7.1.1 Notification of Intent to Comply....................................................................... 7-1

7.1.2 Notification of Intent to Comply Public Meeting and Notice............................. 7-1

7.1.3 RCRA Class 1 Modification Request ................................................................ 7-1

7.1.4 Progress Report.........................................................................................

7.1.5 Permit to Construct/National Emission Standards for Hazardous Air Pollutants ........................................................................................

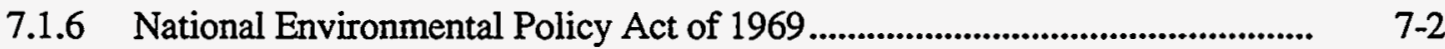

7.2 General Plant Project Construction Project........................................................................... 7-3

7.2.1 Project Definition Phase ......................................................................................... 7-3

7.2.2 Conceptual Design Phase..................................................................................

7.2.3 Title (Detail) Design .............................................................................................

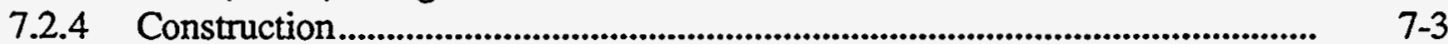

7.2.5 Inspection/Project Transfer ........................................................................

7.3 Recommended Schedule ...........................................................................................

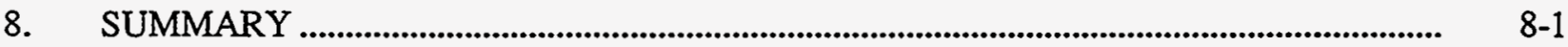

8.1 Technical Risks and Risk Mitigation ................................................................................. 8-1

8.1.1 Adequacy of $\mathrm{HCl}$ Control ................................................................................. 8-1

8.1.2 Adequacy of $\mathrm{Hg}$ Control .................................................................................... 8- 8-1

8.1.3 Adequacy of Dioxin/Furan Control ..............................................................

8.1.4 Safety ............................................................................................................. 8

8.1.5 Facility Assessment ........................................................................................... 8-2

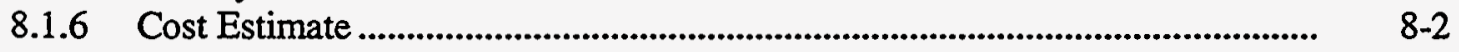

9. REFERENCES .......................................................................................................... 9-1

Appendix A-Mixed Waste Market Evaluation

Appendix B-Material and Energy Balance Calculations

Appendix C-Detailed Cost Estimate

-Appendix D-Engineering Calculations 
Appendix E-Supporting Literature

\section{FIGURES}

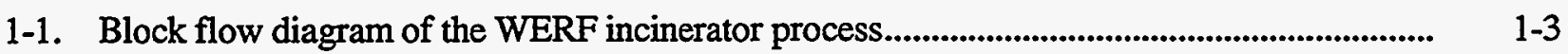

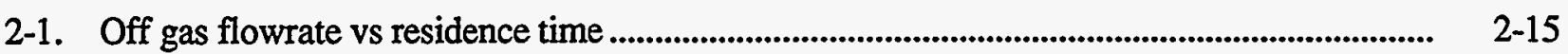

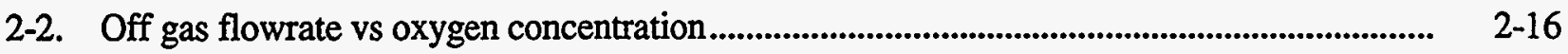

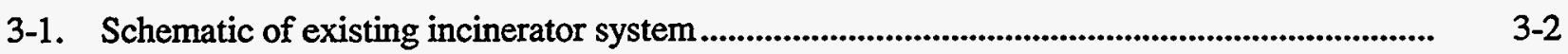

3-2. Existing incinerator air pollution control system .................................................................. $3-4$

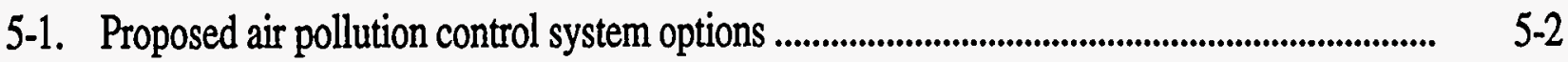

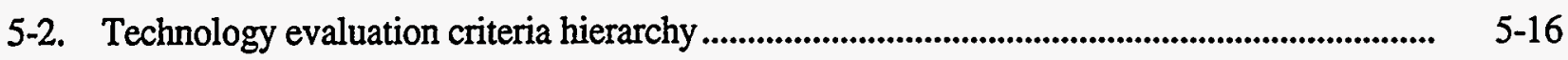

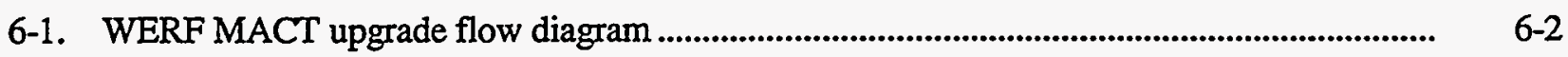

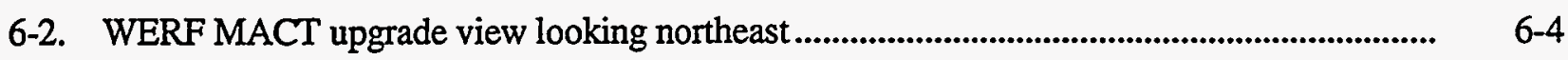

6-3. WERF MACT upgrade view looking southwest.....................................................................

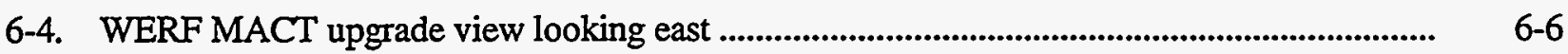

7-1. Recommended WERF MACT compliance schedule .....................................................

\section{TABLES}

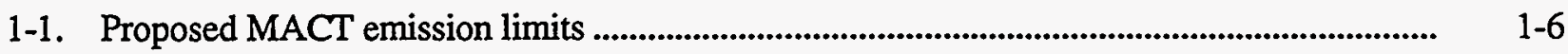

1-2. Baseline control technology strategies to meet MACT emission limits.................................. 1-7

1-3. Proposed continuous air monitoring requirements ................................................................ 1-8

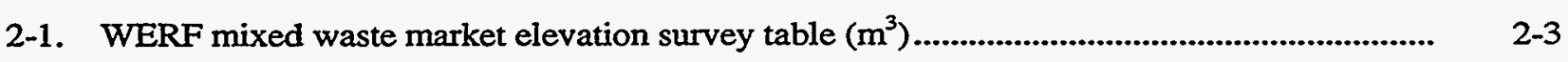

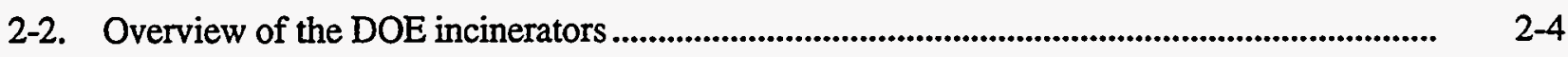

2-3. Current and possible future waste acceptance criteria for MLLW to be incinerated at the WERF..

2-4. Comparison of WERF emissions data to MACT limits

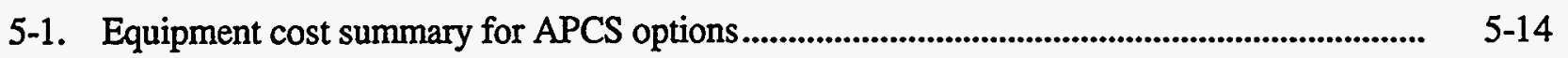

5-2. Criterium decision plus scores............................................................................................ 5 5-17

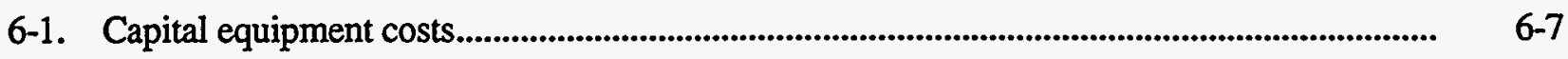


6-2. WERF Operating Schedule Before MACT ................................................................................ 6-9

6-3. Operational Costs before MACT Upgrades ......................................................................... 6-9

6-4. WERF Operating Schedule After MACT .............................................................................. 6-10

6-5. Operational costs after MACT upgrades .................................................................................... 6-10 


\section{ACRONYMS}

acfm

APC

APCS

$\mathrm{CaCl}_{2}$

CEM

CFR

CIF

$\mathrm{D} / \mathrm{F}$

DOE

DRE

dscg

dscm

DSI

EDF

EMI

EPA

GAC

HAP

HEPA

$\mathrm{Hg}$

$\mathrm{HgCl}_{2}$

INEEL

LLW

MACT

MLLW

NEPA

NESHAP

NIC

NODA

$\mathrm{O}_{2}$

PCB

PCC

PIC

PM

'ppvm actual cubic feet per minute

air pollution control

air pollution control systems

calcium chloride

Continuous Emission Monitor

Code of Federal Regulations

Consolidated Incinerator Facility

dioxin/furan

U.S. Department of Energy

destruction and removal efficiency

dry standard cubic feet

dry standard cubic meters

dry sorbent injection

engineering design file

Environmental Management Integration

Environmental Protection Agency

granular activated carbon

Hazardous Air Pollutant

high efficiency particulate air

mercury

mercury dichloride

Idaho National Engineering and Environmental Laboratory

low-level waste

low-volatility metals

maximum achievable control technologies

mixed low-level waste

National Environmental Policy Act

National Emission Standards for Hazardous Air Pollutants

Notice of Intent to Comply

Notice of Data Availability

\section{Oxygen}

polychlorinated biphenyl

primary combustion chamber

product of incomplete combustion

particulate matter

parts per million by weight 


$\begin{array}{ll}\text { PVC } & \text { polyvinyl chloride } \\ \text { RCRA } & \text { Resource Conservation Recovery Act } \\ \text { SCC } & \begin{array}{l}\text { secondary combustion chamber } \\ \text { spray dry adsorber }\end{array} \\ \text { SDA } & \text { Site Treatment Plan } \\ \text { STP } & \text { semi-volatile metals } \\ \text { SVM } & \text { Toxicity Characteristic Leaching Procedure } \\ \text { TCLP } & \text { toxic equivalent } \\ \text { TEQ } & \text { total hydrocarbons } \\ \text { THC } & \text { Toxic Substances Control Act } \\ \text { TSCA } & \\ \text { WAC } & \text { waste acceptance criteria } \\ \text { WERF } & \text { Waste Experimental Reduction Facility }\end{array}$




\section{WERF MACT Feasibility Study}

\section{INTRODUCTION}

The Waste Experimental Reduction Facility (WERF) at the Idaho National Environmental and Engineering Laboratory (INEEL) currently incinerates low-level waste (LLW) and mixed low-level waste (MLLW). New emission standards reflecting the performance of maximum achievable control technologies (MACT) have been proposed to afford increased protection to human health and the environment over existing Resource Conservation and Recovery Act (RCRA) standards. These standards include more strict emission limits for a variety of pollutants as well as the use of continuous emissions monitoring systems to confirm compliance. The following sections provide a general overview of the incineration process at the WERF, explain the proposed MACT emission limits, and identify areas where the WERF would fail to comply with the proposed limits, unless changes were made in its air pollution control system (APCS).

\subsection{Problem Statement}

This technical feasibility study was undertaken to determine answers to the following questions:

- What technologies are available and necessary to upgrade the WERF Incinerator Air Pollution Control System to meet the intent of the proposed MACT rule?

- Due to the anticipated MACT implementation schedule, can this upgrade be accomplished within the restraints of the General Plant Project funding limit (\$5 M)?

- What can be done to expand waste acceptance criteria at WERF?

- What is the recommended strategy?

\subsection{WERF Background}

The WERF was constructed in the converted Special Power Excursion Reactor No. 3 building and commenced operations in 1982. Initially, the purpose of the project was to demonstrate that commercially available technologies could be used for managing the LLW generated by the nuclear facilities located at the INEEL. The pilot-scale facility data obtained from this experiment was to be used in developing future production-scale waste processing facilities.

In the late 1980 s, additional experiments were performed to evaluate the ability of WERF to treat MLLW. Treatment involved reducing the waste toxicity and volume via incineration. Due to the success of these experiments, WERF has developed into a facility capable of treating complex-wide MLLW. This includes waste currently being generated and legacy waste from prior U.S. Department of Energy (DOE) operations. From October 1984 to February 1991, the WERF incinerator completed nearly 10,000 hours of operation without significant incidences or failures. During that time, more than $325,000 \mathrm{ft}^{3}$ (greater than 1.4 million lbs) of waste was incinerated with an average volume reduction ratio of 200:1. Most of the waste incinerated was LLW.

In February 1991, the WERF underwent a planned shutdown to perform major modifications to upgrade facility operating documentation and re-baseline the facility configuration and administrative management programs. The resumption of operations project, known as the WERF Restart Project, was classified as a DOE Type 3 restart: a restart following a planned shutdown. This shutdown involved a 
significant period of time, and substantial plant modifications were required for future programmatic work and safety enhancements.

During the WERF Restart Project, major construction upgrades were completed that provided operational enhancements and extended the operating life of the facility. These included relocation of the compaction and sizing processes into a larger building, completion of construction on the WERF Waste Storage Building, installation of incinerator offgas and sizing/compaction facility isokinetic sampling systems, and incinerator primary chamber refractory replacement. Since the resumption of WERF operation in July 1995 , the incinerator has treated $572,000 \mathrm{lbs}$ of LLW; $44,000 \mathrm{lbs}$ of characteristic MLLW; and 295,000 lbs of listed MLLW.

\subsubsection{Incineration System Description}

The WERF incinerator is a dual-chambered (primary and secondary chamber), controlled air incinerator, manufactured by Environmental Control Products of Charlotte, North Carolina, with a thermal capacity of $6.5 \mathrm{MBtu} / \mathrm{hr}$. Solid waste is gravity fed to the primary chamber where it is oxidized in an oxygen-deficient environment. This sub-stoichiometric environment is maintained by controlling the air introduced into the primary chamber so that volatile materials can be driven off in a controlled manner, and particulate emissions are minimized.

The WERF APCS currently uses a three-step process for cooling the combustion gas exiting the secondary chamber prior to entering downstream air pollution control (APC) components. In the first step, outside air is mixed with the offgas just downstream of the secondary chamber to reduce the offgas temperature. The offgas then passes through the air-to-air heat exchanger for additional cooling, and after exiting the heat exchanger, more ambient air is added before it enters the baghouse system. The WERF baghouse system consists of two pulse-jet type baghouses. They are installed in parallel with a common outlet plenum. The offgas exits the baghouse and flows into a high efficiency particulate air (HEPA) filter designed to remove submicron particles that the baghouse filters cannot capture. Figure 1-1 is a block flow diagram for the existing incinerator APCS.

The offgas flows from the HEPA filters through a duct system equipped with continuous emission monitors (CEMs). The CEMs are used to measure the offgas oxygen $\left(\mathrm{O}_{2}\right)$, carbon monoxide (CO), and hydrochloric acid $(\mathrm{HCl})$ concentrations. The CEMs are used for automatic waste feed shut-offs if offgas emission concentrations are greater than permitted values. The offgas then flows into two induced draft fans. The fans maintain a negative pressure within the incinerator and APCS and transports the offgas from the incinerator through the APCS and into the north stack, which is the emission point for the system. 


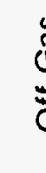

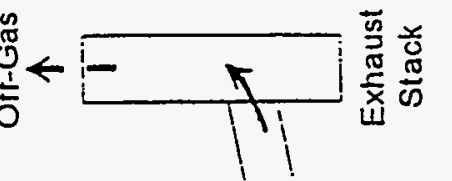

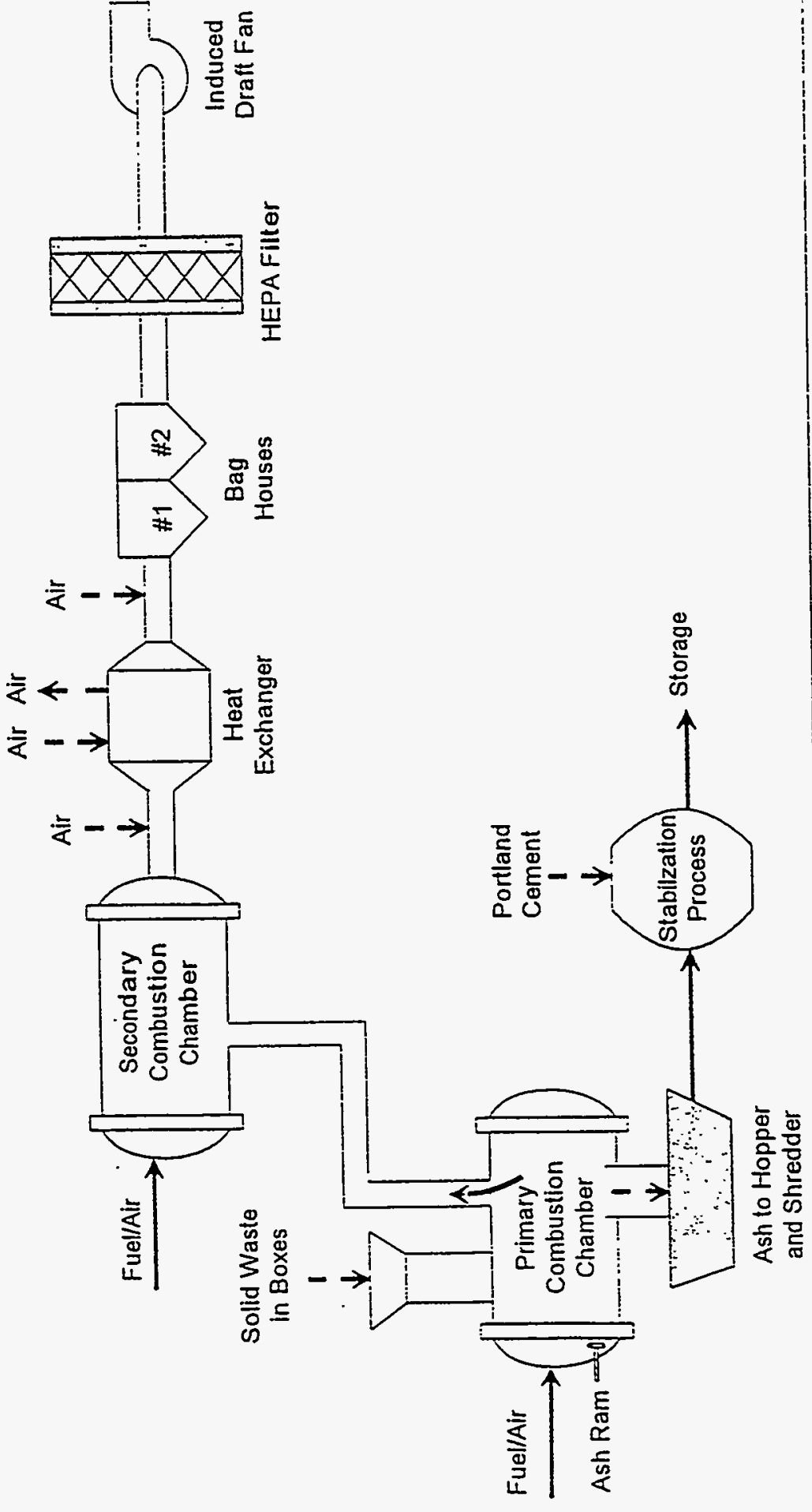

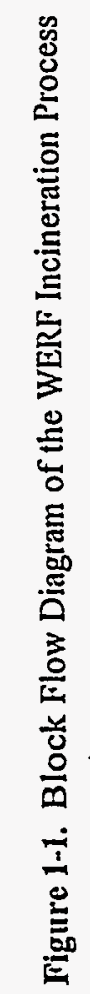




\subsubsection{Incineration Campaign Schedule}

The incinerator is normally operated once per month in a 10 to 20 day campaign. While processing, characteristic MLLW and LLW can be burned. However, listed MLLW must be treated exclusively in dedicated campaigns. Since the cost of disposing of listed waste, including incinerator ash, is very expensive, exclusive campaigns avoid generating more listed ash than necessary.

Presently, the WERF is incinerating both INEEL-generated MLLW and other DOE facility-generated MLLW. Off-site MLLW is subject to the State of Idaho Governor's Agreement that "Primary Treatment" (incineration) will be accomplished within 6 months of receiving the waste, and the waste residues are required to leave the State of Idaho no later than 6 months after completion of the primary treatment.

\subsection{Regulatory Background and Requirements}

Currently, the WERF is classified as a RCRA interim status facility. The RCRA Part A permit application was submitted for the WERF in September 1990, and the State of Idaho recognized interim status in March 1991. A RCRA Part B Permit application was submitted to the State of Idaho in October 1992. As required in the RCRA regulations, an incinerator must demonstrate environmental compliance in a controlled series of tests called a trial burn. Trial burn tests are designed to operate the incinerator at the expected upper and lower operating ranges and are specifically set up to evaluate an incinerator's waste treatment capabilities under the worst possible operating conditions.

A trial burn plan, documenting the test protocol and proposed test conditions, was submitted to the State of Idaho in November 1992, with a goal of completing the trial burn in 1994. ${ }^{1}$ However, the trial burn was rescheduled until 1997 when two series of tests were performed to establish the WERF operating envelope. These tests were called the Low Temperature Trial Bum and the High Temperature Trial Burn.

The results for the Low Temperature Trial Burn showed that the WERF successfully met the applicable RCRA requirements. However, during the High Temperature Trial Burn, the incinerator attained an average destruction and removal efficiency (DRE) for principle organic hazardous constituents of $99.99 \%$, but failed to achieve this required value on one of three tests. Additionally, the High Temperature Trial Burn data indicated a dioxin/furan (D/F) emissions rate that would have failed the screening level risk assessment for air toxics. The WERF incinerator has continued to operate under interim status and operating conditions have been modified to eliminate excessive $\mathrm{D} / \mathrm{F}$ formation. The High Temperature Trial Burn was repeated in August 1998, and preliminary test results indicate that the WERF incinerator successfully met the test objectives.

\subsubsection{Proposed Rule}

On April 19, 1996, the Environmental Protection Agency (EPA) published "Hazardous Waste Combustors; Revised Standards; Proposed Rule"2 under the joint authority of the RCRA and the Clean Air Act. These standards reflect the performance of MACT, as specified by the Clean Air Act. The MACT standards were developed to increase protection to human health and the environment. This proposed rule addresses the following:

- Integrating the monitoring, compliance testing, recordkeeping, and permitting requirements of RCRA and the Clean Air Act. 
- Setting emissions standards for D/F, Hg, two semi-volatile metals (SVM) (cadmium and lead), four low-volatility metals (LVM) (antimony, arsenic, beryllium, and chromium), combined hydrochloric acid and chlorine gas, particulate matter (PM), and two surrogates for non-D/F organic hazardous air pollutants (HAPs) (carbon monoxide and hydrocarbons).

- Using CEMs and operating parameter limits based upon comprehensive performance tests to comply with the proposed MACT standards.

Comments on this proposed rule were sought from the public and affected industry. The DOE Headquarters submitted a consolidated response that addresses impacts to current and future DOE waste management activities and strategies, and in particular, management of mixed waste involving thermal treatment. Key issues discussed include the following:

- Appropriateness of creating a subcategory for mixed-waste incinerators and waste feed variability.

- Applicability of the new MACT standards to hazardous waste combustors used for research, development, demonstration, or treatability studies.

- Difficulties with CEMs for mercury and PM in mixed waste incinerators.

- Impact on Federal Facility Compliance Act requirements and schedules.

\subsubsection{Notice of Data Availability}

The "Revised Technical Standard for Hazardous Waste Combustion Facilities; Proposed Rule"3 was published on May 2, 1997. This Notice of Data Availability was issued to inform the public of significant changes that the EPA is considering on aspects of the proposed regulation based on public comments and new information. It provides data from various EPA tests as supporting information and provides demonstrated evidence that the proposed limits can be met with existing technologies. The most significant departure from current regulations is that the proposed standards regulate the HAPs based upon the concentration emitted instead of the previous philosophy and practice of air dispersion modeling and risk analysis.

The second publication also addresses DOE's comments. The EPA is still investigating whether DOE incinerators pose unique implementation and compliance problems. The EPA is still considering several options for the final rule, including no subcategorization, subcategorization, or deferral of MACT standards for mixed waste incinerators (with the applicable RCRA rules continuing to apply).

Under the no subcategorization option, the EPA would rule that the MACT controls and emissions standards applicable to other incinerators are appropriate for DOE mixed waste incinerators. With subcategorization for mixed waste incinerators, the EPA would rule that because of unique design and operating features, the MACT controls or emissions standards identified for other incinerators are not appropriate for mixed-waste incinerators. Under this option, MACT standards unique to mixed-waste incinerators would be developed, and special compliance requirements would be defined.

Under the deferral option, the EPA would rule that it does not have enough data and resources to make an appropriate MACT determination on the mixed-waste incinerators in time to meet the schedule for the Hazardous Waste Combustor rulemaking. If this option were chosen, regulation of mixed waste incinerators would be deferred to the Phase II rulemaking. 
The EPA deferred a final decision on these options until the final rule, stating that it will continue investigation on the appropriate regulatory options. However, speculations and technical experts within the industry believe it is likely that the EPA will most likely choose the no subcategorization option because the current political atmosphere supports this speculation, which is not in favor of incineration and also because technology has evolved to provide for emissions and monitoring controls capable of meeting the proposed regulatory limits.

\subsubsection{MACT Proposed Emission Limits (May 2, 1997)}

The Notice of Data Availability proposes concentration-based standards for HAPs or easily detected HAP surrogates (Table 1-1). EPA re-evaluated the LVM HAP category and determined that antimony may not warrant direct control; the MACT PM standard may serve as an adequate surrogate.

\subsubsection{MACT Emission Controls}

Also, within the proposed regulations, the EPA identifies currently installed emissions control technologies upon which the EPA MACT emissions limits were based. This does not mean that these technologies must be used, but the technology chosen must be able to meet the MACT emission limits (Table 1-2).

Table 1-1. Proposed MACT emission limits.

\begin{tabular}{|c|c|}
\hline HAP or HAP Surrogate & $\begin{array}{l}\text { Proposed Standard } \\
\text { (corrected to } 7 \% \mathrm{O}_{2} \text { ) }\end{array}$ \\
\hline Dioxin/furans & $\begin{array}{l}0.20 \mathrm{ng} \text { toxic equivalent }(\mathrm{TEQ}) / \mathrm{dscm} \text { or } \\
0.40 \mathrm{ng} \mathrm{TEQ} / \mathrm{dscm} \text { and } \leq 400^{\circ} \mathrm{F} @ \mathrm{PM} \text { control } \\
\text { device }\end{array}$ \\
\hline Particulate matter & $0.015 \mathrm{gr} / \mathrm{dscf}$ \\
\hline Mercury & $40 \mu \mathrm{g} / \mathrm{dscm}$ \\
\hline Semi-volatile metals $(\mathrm{Cd}, \mathrm{Pb})$ & $100 \mu \mathrm{g} / \mathrm{dscm}$ \\
\hline Low-volatile metals (As, $\mathrm{Be}, \mathrm{Cr}$ ) & $55 \mu \mathrm{g} / \mathrm{dscm}$ \\
\hline Hydrochloric acid/chlorine & 75 ppmv \\
\hline Carbon monoxide & $100 \mathrm{ppmv}$ \\
\hline Hydrocarbon & 10 ppmv \\
\hline
\end{tabular}


Table 1-2. Baseline control technology strategies to meet MACT emission limits.

HAP or HAP

Surrogate

MACT Basis

Dioxin/furans

Rapid quench by wet scrubber

Particulate matter

1. Fabric filter $\mathrm{w} / \mathrm{air} / \mathrm{cloth}$ ratio of $10 \mathrm{acfm} / \mathrm{ft}^{2}$

2. Venturi scrubber and ionizing wet scrubber in series

Mercury

Wet scrubber w/ MTEC ${ }^{\mathrm{a}} \leq 40 \mu \mathrm{g} / \mathrm{dscm}$

Semi-volatile metals

1. Venturi scrubber w/ MTEC $\leq 170 \mu \mathrm{g} / \mathrm{dscm}$

2. Electrostatic precipitator and wet scrubber in series w/MTEC $\leq$ $5,800 \mu \mathrm{g} / \mathrm{dscm}$

3. Venturi scrubber and ionizing wet scrubber in series $w / M T E C \leq$ $49,000 \mu \mathrm{g} / \mathrm{dscm}$

Low-volatility metals

1. Venturi scrubber $w / M T E C \leq 1,000 \mu g / d s c m$

2. Ionizing wet scrubber $w / M T E C \leq 6,200 \mu \mathrm{g} / \mathrm{dscm}$

Hydrochloric

Wet scrubber w/ feed rate controls

acid/chlorine

Carbon monoxide

Good combustion control

Hydrocarbon

Good combustion control

a. MTEC-maximum theoretical emissions concentration.

\subsubsection{Continuous Emissions Monitoring}

The proposed regulation also requires continuous indication of the emissions rates for several of the regulated HAPs or HAP surrogates. If a philosophy similar to the existing RCRA regulations is adopted, these CEMs will be installed with feedback loop control circuitry, which will provide automatic waste feed cutoffs to the process when monitored parameters exceed regulated limits. Although uncertain at this time, it is reasonable to believe that the final rule will require CEMs feedback for process control.

Table 1-3 summarizes the proposed CEMs requirements. Note that only three of the HAP/HAP surrogate CEMs are required by the proposed standards; the others are listed to show their proposed required averaging periods, if used. Manual testing averaging periods are given for those analytes that do not require CEMs. 
Table 1-3. Proposed continuous air monitoring requirements.

Continuous Air Monitoring

System Averaging Period

Hazardous Air Pollutant or Surrogate

Particulate material (required)

Carbon monoxide (required)

Total hydrocarbons (required)

Mercury

Semi-Volatile metals

Low-Volatile metals

Hydrochloric acid/chlorine (hrs)

Manual Testing Averaging Period (hrs)

$\begin{array}{cc}6^{2} & - \\ 1 & - \\ 1 & - \\ 10 & 1-2 \\ 10 & 2 \\ 10 & 2 \\ 1 & 1\end{array}$

a. The proposed averaging for particulate material continuous air monitoring system was revised from $2 \mathrm{hrs}$ to $6 \mathrm{hrs}$ at the Chemical Manufacturer's Association meeting for stakeholders in Washington, D.C., on June 4, 1998.

\subsubsection{Final Rule}

On June 19, 1998, the Federal Register published a final rule, ${ }^{4}$ which resolves some elements of the MACT standards for air emissions originally proposed on April 19, 1996. In the final rule, a new provision is finalized that streamlines the existing RCRA permit modification process to make it easier for facilities to make changes to their existing permits when modifying their equipment or operation in order to comply with the MACT standards. However, this portion of the rule must be adopted by a RCRA-authorized state, such as Idaho, before it can be applied in that state. The notification requirements for sources that intend to comply with the MACT standards are also finalized. These requirements are discussed in Section 7 of this report. 


\section{MARKET ANALYSIS FOR MIXED LOW-LEVEL WASTE INCINERATION AT THE WERF}

\subsection{Mixed Waste Market Evaluation}

The mixed waste treatment market is highly volatile. Individual DOE sites are driven to treat and dispose of their mixed waste cheaper and faster. The Toxic Substances Control Act incinerator in Oak Ridge, Tennessee, has treated over 18 million lbs of primarily liquid mixed waste since 1991. The WERF incinerator has treated over 340,000 lbs of mostly solid mixed waste since starting mixed waste processing in 1995. The Consolidated Incinerator Facility (CIF) at the Savannah River Site is on-line and treating Savannah River Site-generated liquid mixed waste. Implementation of the DOE "Paths to Closure Plan" has accelerated the generation of mixed waste available for treatment, as has the closure of DOE sites and storage facilities. In light of this dynamic market, the question, "Is there enough mixed waste to operate the upgraded WERF incinerator efficiently and at capacity?" must be answered. Based on the analysis outlined below the answer is YES; there is enough mixed waste in the system to operate the WERF incinerator at capacity for 10 years.

\subsubsection{Mixed Waste Inventory Report-1995}

The Mixed Waste Inventory Report- $1995^{6}$ identified the current inventories and future generation rates of mixed waste across the DOE complex. The report was used as the basis for the individual sites Site Treatment Plans. These data were screened only to identify waste streams that were non-transuranic, non-asbestos, non-soil, contact-handled, and low-ash content, i.e., incinerable waste. The waste database reviewed by a WERF bum engineer and categorized as either a definite or possible candidate for WERF incineration. The results are summarized in Table A-1.

The review concluded that the WERF facility had the capability to potentially treat $25 \%\left(3,772 \mathrm{~m}^{3}\right)$ of the incinerable mixed waste in inventory and over $90 \%\left(7,310 \mathrm{~m}^{3}\right)$ of the mixed waste to be generated in the next five years. Based on this preliminary review of the mixed waste market, the INEEL sought to supplement the excess mixed waste treatment capacity with off-site waste.

\subsubsection{INEEL Site Treatment Plan}

In the fall of 1997, the INEEL revised the Site Treatment Plan to include 91 additional mixed waste streams totaling $741 \mathrm{~m}^{3}$ of off-site inventory and $1,570 \mathrm{~m}^{3}$ of five-year generation. These additions were made at the request of the generating sites during the 1997 DOE Incinerator System conference. The offsite waste currently approved for incineration is summarized in Table A-2. There is $813 \mathrm{~m}^{3}$ in inventory and $1,648 \mathrm{~m}^{3}$ to be generated in five years from $27 \mathrm{DOE}$ sites approved by the Sate of Idaho for incineration at WERF.

The current STP shows $411 \mathrm{~m}^{3}$ of on-site mixed waste inventory for incineration and a five-year generation of $160 \mathrm{~m}^{3}$ (Table A-3). The current inventory does not reflect $38 \mathrm{~m}^{3}$ that will be treated within one year of generation nor the incinerable portion of TEC-698 $\left(704 \mathrm{~m}^{3}\right)$, or the ER-TAN-624 polychlorinated biphenyl (PCB) waste $\left(880 \mathrm{~m}^{3}\right)$. The total on-site incinerable waste is $2,193 \mathrm{~m}^{3}$, with an additional $2,461 \mathrm{~m}^{3}$ from off-site, brings the total backlog of mixed waste to incinerate at WERF prior to MACT upgrade to $4,654 \mathrm{~m}^{3}$.

The current STP covers 1998 and the following five years generation of mixed waste. It is reasonable to assume that generation of mixed waste will continue at the same rate or increase as DOE facilities are closed. The conservative assumption would predict that the current generators would 
generate an additional $5000 \mathrm{~m}^{3}$ between 2004 and 2010 . Therefore, the market for mixed waste treatment at WERF between 1999 and 2010 would be approximately $10,000 \mathrm{~m}^{3}$.

\subsubsection{Environmental Management Integration}

The Environmental Management Integration (EMI) project met with various DOE sites representatives to prepare mixed waste disposition maps in 1998. The incinerable waste destined for each DOE mixed waste incinerator (TSCA, CIF, and WERF) are summarized in Table A-4. The EMI disposition maps show approximately $847 \mathrm{~m}^{3}$ of waste planned for treatment at WERF. This is approximately one-third of the volume identified by the INEEL site treatment plan. The differences can be traced to the uncertainty surrounding four waste streams. Three from Hanford, RL-601-01 Mixed Waste Debris $\left(866 \mathrm{~m}^{3}\right)$, RL-ALO Organic Absorbed Liquids $\left(309 \mathrm{~m}^{3}\right)$, RL-LPO Organic Lab Packs $\left(222 \mathrm{~m}^{3}\right)$, and one from Rocky Flats, RF-W071-Granulated Activated Carbon $\left(150 \mathrm{~m}^{3}\right)$. Both sites have requested that the original inventories remain in the INEEL STP since treatment at WERF is still the probable destination for this material. Considering these exceptions, the EMI mixed waste disposition maps and the INEEL STP are in acceptable agreement.

\subsubsection{Mixed Waste Focus Area}

The Mixed Waste Focus Area published a MACT System Integration Study Draft Status Report dated October 23,1998. ${ }^{7}$ This report examined the impacts that the proposed MACT rule would have on the three DOE mixed waste incinerators. The report identifies the compliance options for each incinerator, the effects on the mixed waste planned for treatment, and capital cost estimates. The mixed waste identified for treatment is summarized by the EMT program waste disposition maps. Waste streams destined for the three incinerators are shown in Tables A-5, A-6, and A-7. Table A-7 contains some but not all the waste in the INEEL STP destined for WERF but not in the most recent EMI disposition maps, and is subject to the same limitations as the original EMI data, discussed above.

The Mixed Waste Focus Area also evaluated the waste streams that could be treated if WERF was upgraded as proposed TSCA while leaving the incinerator and CIF unmodified. This evaluation is summarized in Table A-8. The waste planned for the CIF incinerator contains approximately $7,567 \mathrm{~m}^{3}$ of mixed waste that CIF would not treat if not upgraded for MACT and $103 \mathrm{~K} \mathrm{~m}^{3}$ of low-level waste that was planned to be incinerated but will exceed the Hg limits. Thus, if a MACT upgrade is not completed for $\mathrm{CIF}$ or if that upgrade required a line item construction project, nearly $110 \mathrm{~K} \mathrm{~m}^{3}$ of waste would be available for treatment in the WERF incinerator. A similar situation exists for the TSCA incinerator. Without the MACT upgrade, the TSCA incinerator would be unable to treat approximately $60 \mathrm{~K} \mathrm{~m}^{3}$ of mixed waste, primarily waste with $\mathrm{Hg}$ above the incinerator limits of MACT.

\subsubsection{Conclusions}

When the various mixed waste databases are reconciled (as summarized in Table 2-1), it shows that approximately $5,000 \mathrm{~m}^{3}$ of waste is available for WERF treatment in the next five years. By a conservative extrapolation to 2010 , it appears that an additional $5,000 \mathrm{~m}^{3}$ would be available for WERF, not considering significant waste volume from the other incinerator sites if they are not upgraded. This totals approximately $10,000 \mathrm{~m}^{3}$. The upgraded capacity of WERF will range from $700 \mathrm{~m}^{3} / \mathrm{yr}$ to $1300 \mathrm{~m}^{3} / \mathrm{yr}$. Considering these assumptions, there is enough suitable mixed waste in the DOE system to operate WERF at capacity for at least 10 years, regardless of the MACT compliance status for CIF and the TSCA incinerator. 
Table 2-1. WERF mixed waste market elevation survey table $\left(\mathrm{m}^{3}\right)$.

\begin{tabular}{lccc}
\hline \multicolumn{1}{c}{ Source } & $\begin{array}{c}\text { Incinerable Mixed } \\
\text { Waste Inventory }\end{array}$ & $\begin{array}{c}\text { 5yr Generator } \\
\text { Volume }\end{array}$ & Total \\
\hline Mixed Waste Inventory & & & \\
Report 1995 & 3772 & 7314 & 1,1086 \\
INEEL Site Treatment Plan & & & \\
Onsite (with addendums) & 2033 & 160 & 2,193 \\
Off-site & 813 & 1648 & 2,461 \\
STP TDTM & 2846 & 1808 & 4,654 \\
EMI Disposition Maps & & & 847 \\
Mixed Waste Focus & & & 1,630 \\
\hline
\end{tabular}

\subsection{DOE Incinerator Features and MACT Compliance Status}

The following section has been excerpted from MACT System Integration Study Draft Status Report. ${ }^{7}$ in its entirety. The major features are summarized in Table 2-2 for each of the three DOE incinerators (CIF, TSCA, and WERF). This table also indicates current MACT compliance status for the three incinerators. Although the revised proposed limits from the May 2, 1997, Notice of Data Availability (NODA) ${ }^{3}$ (62 FR 24212) are assumed to be valid, there is still some uncertainty in the pollutant concentration limits that will be promulgated. The D/F limit may increase from the revised proposed limit of 0.2 to $0.4 \mathrm{ng} / \mathrm{dscm}$ TEQ at $7 \% \mathrm{O}_{2}$ ). The $\mathrm{Hg}$ limit may increase to $100 \mu \mathrm{g} / \mathrm{dscm}$ (at $7 \%$ $\mathrm{O}_{2}$ ) or higher from the revised proposed limit of $40 \mu \mathrm{g} / \mathrm{dscm}\left(\right.$ at $7 \% \mathrm{O}_{2}$ ). Administrative limits of approximately one-half of the revised proposed limits were assumed for this study for calculating target offgas concentrations, control efficiencies, and feed limits. This approach was described in the May 2,1997, NODA as necessary to account for normal operational variability (not including feed uncertainty).

Feed concentration uncertainties would further reduce the actual operating limits. All three facilities would require some combination of feed limit, operational, or equipment modifications to comply with the MACT rule. These facilities may be made to comply with the proposed regulation through some combination of the following:

- Lower feed limits (requiring some combination of improved feed characterization, segregation, pretreatment, blending, staging, lower feed-rates, and elimination of some feeds) for constituents such as $\mathrm{Hg}, \mathrm{Cl}$, solids, sludges, and metals that potentially cause emissions to exceed MACT limits.

- Make operational modifications, including use of feed or fuel additives, as applicable, to optimize performance without capital equipment modifications

- Make capital equipment modifications

Some equipment and operating modifications would be necessary for all three facilities if they intend to continue operation with existing feed limits and waste acceptance criteria (WAC). The 
Table 2-2. Overview of the DOE incinerators., ${ }^{2, b, c, d, e, f, g, h}$

\begin{tabular}{|c|c|c|c|}
\hline & CIF, Savannah River Site & $\begin{array}{l}\text { TSCA incinerator, East Tennessee } \\
\text { Technical Park }\end{array}$ & WERF, INEEL \\
\hline Treatment system & Dual chamber rotary kiln & Dual chamber rotary kiln & Dual chamber controlled air \\
\hline $\begin{array}{l}\text { Designed and } \\
\text { permitted feed } \\
\text { materials }\end{array}$ & $\begin{array}{l}\text { Incinerable solid and liquid } \\
\text { hazardous, LLW, and MLLW from } \\
\text { SRS. Solid waste is repackaged } \\
\text { into boxes to feed to kiln. Liquids } \\
\text { are fired through a bumer in the } \\
\text { kiln, except for Defense Waste } \\
\text { Processing Facility benzene waste, } \\
\text { which is fired through a bumer in } \\
\text { the SCC. }\end{array}$ & $\begin{array}{l}\text { Incinerable solid, sludge, and liquid } \\
\text { hazardous, } L \text {, mixed, and PCB } \\
\text { wastes. Solid and sludge wastes are } \\
\text { box-fed to the kiln, and liquid } \\
\text { wastes are injected through bumers. } \\
\text { Bulk-feeding of solids is not } \\
\text { presently done. }\end{array}$ & $\begin{array}{l}\text { Box-fed hazardous, LL, and mixed } \\
\text { incinerable solid, sludge, and liquid } \\
\text { waste from the INEEL and other } \\
\text { DOE sites. Waste is typically } \\
\text { repackaged into boxes to feed. } \\
\text { Liquids are packaged in limited } \\
\text { quantities in closed plastic bottles or } \\
\text { absorbed onto corncob sorbent, and } \\
\text { placed in boxes. Liquid waste feed } \\
\text { system was designed and partially } \\
\text { installed in early } 1990 \text { 's, but is not } \\
\text { operational. }\end{array}$ \\
\hline Capacity (MMBtw/hr) & 39.26 (permitted total) & $30 \max$ & 5 (solid waste), 6.5 (total) \\
\hline Waste Feedrate, lbs $/ \mathrm{hr}$ & $\begin{array}{l}2,426 \text { total for all waste and } \\
\text { auxiliary fuel }\end{array}$ & $\begin{array}{l}2,300 \text { (TSCA permit limit) } 2,400 \\
\text { (RCRA permit limit) }\end{array}$ & 400 (permit limit) \\
\hline $\begin{array}{l}\text { Treatment } \\
\text { temperatures, } C(F)\end{array}$ & $\begin{array}{l}\text { PCC: } 1,400-1,832^{\circ} \mathrm{F}(760- \\
1,000 \mathrm{C}) \\
\text { SCC: } 1,600-2,012^{\circ} \mathrm{F}(870- \\
1,100 \mathrm{C})\end{array}$ & $\begin{array}{l}\text { PCC: }>1,565^{\circ} \mathrm{F}\left(>850^{\circ} \mathrm{C}\right) \\
\text { SCC: } 1,875-2,190^{\circ} \mathrm{F}(1,020- \\
\left.1,200^{\circ} \mathrm{C}\right)\end{array}$ & $\begin{array}{l}\text { PCC: } 1,600-2,200^{\circ} \mathrm{F}(870- \\
1,200 \mathrm{C}) \\
\text { SCC: } 1,700-2,200^{\circ} \mathrm{F}(930- \\
1,200 \mathrm{C})\end{array}$ \\
\hline $\begin{array}{l}\text { SCC design residence } \\
\text { time, seconds }\end{array}$ & $>2$ & 4 & 2 \\
\hline $\begin{array}{l}\text { Off gas flowrates, } \\
\text { scfm }\end{array}$ & $\begin{array}{l}\text { SCC outlet: }-6,400-9,000 \\
\text { Stack: } \sim 18,000 \text { (higher due to added } \\
\text { water vapor used in evaporative } \\
\text { cooling and scrub solution atomizing } \\
\text { steam) }\end{array}$ & $\begin{array}{l}\text { SCC outlet: } \sim 10,000 \\
\text { Stack: } \sim 20,000 \text { (higher due to added } \\
\text { water vapor used in evaporative } \\
\text { cooling) }\end{array}$ & $\begin{array}{l}\text { SCC outlet: } \sim 1,800 \\
\text { Stack: }-6,600 \text { (higher due to added } \\
\text { cooling air) }\end{array}$ \\
\hline \multicolumn{4}{|l|}{ APCS: } \\
\hline Off gas cooling & $\begin{array}{l}\text { Scrub solution evaporative quench } \\
\text { to a saturation temperature of } \\
\sim 180^{\circ} \mathrm{F}\end{array}$ & $\begin{array}{l}\text { Water spray evaporative quench to a } \\
\text { saturation temperature of } \sim 180^{\circ} \mathrm{F}\end{array}$ & $\begin{array}{l}\text { Air dilution to } \sim 1,250^{\circ} \mathrm{F} \text {; gas/air heat } \\
\text { exchanger to }>625^{\circ} \mathrm{F} \text {; air dilution to } \\
<395^{\circ} \mathrm{F}\end{array}$ \\
\hline $\begin{array}{l}\text { PM and radiological } \\
\text { control }\end{array}$ & $\begin{array}{l}\text { Steam atomized scrubber removes } \\
\text { PM and acid gases; cyclone } \\
\text { separator provides liquid-gas } \\
\text { separation of scrubbing mixture; }\end{array}$ & $\begin{array}{l}\text { Venturi scrubber removes PM } 1 \mu \mathrm{m} \\
\text { or greater and a portion of the } \mathrm{HCl} \\
\text { followed by a demister to remove } \\
\text { entrained water droplets. }\end{array}$ & $\begin{array}{l}\text { Baghouse with } 27,000 \text { dscm/h } \\
\text { ( } 16,000 \text { scfm) capacity; removes } \\
99 \% \text { of the dust; preceded by spark } \\
\text { arrestors. }\end{array}$ \\
\hline Acid gas control & $\begin{array}{l}\text { mist eliminator removes entrained } \\
\text { water droplets. }\end{array}$ & $\begin{array}{l}\text { Cross-flow packed bed scrubber } \\
\text { removes } \mathrm{HCL}, \mathrm{HF} \text {, and } \mathrm{SO}_{2}\end{array}$ & $\begin{array}{l}\text { None by design, although the } \\
\text { baghouse achieves some inherent } \\
\text { dry srubbing (19-34\%). A spray } \\
\text { dryer was designed and partially } \\
\text { installed in early 1990's, but not } \\
\text { operational. }\end{array}$ \\
\hline NOx control & None & None & None \\
\hline Final filtration & Prefilters and double HEPAs & $\begin{array}{l}\text { Ionizing wet scrubber removes fine } \\
\text { particulate less than } 1 \mu \mathrm{m}\end{array}$ & $\begin{array}{l}\text { Single-stage prefilters and single- } \\
\text { stage HEPAs }\end{array}$ \\
\hline Off gas CEM & $\begin{array}{l}\text { Flowrate, } \mathrm{O}_{2}, \mathrm{CO} \text {, radioactivity, } \\
\text { opacity }\end{array}$ & Flowrate, $\mathrm{O}_{2}, \mathrm{CO}, \mathrm{CO}_{2}$, opacity & $\begin{array}{l}\mathrm{O}_{2}, \mathrm{CO}, \mathrm{HCl} \text {, and combustible gas, } \\
\text { radioactivity }\end{array}$ \\
\hline $\begin{array}{l}\text { Dioxins/Furans, } \\
0.2 \text { ng/dscm TEQ }\end{array}$ & $\begin{array}{l}\text { While D/F levels exceeded MACT } \\
\text { limits in the trial bum }(0.5-3.2 \\
\text { ng/dscm TEQ), this system, when } \\
\text { optimized, should result in } \\
\text { sufficiently low D/F levels. CIF } \\
\text { staff is working to resolve this. }\end{array}$ & .. & $\begin{array}{l}\text { For estimated D/F levels ranging } \\
\text { from } 1-10 \text { ng/dscm, need } 90-99 \% \\
\text { reduction below current levels. } \\
\text { APCS modifications are the only } \\
\text { option for compliance. }\end{array}$ \\
\hline
\end{tabular}


Table 2-2. (continued).

\begin{tabular}{|c|c|c|c|}
\hline & CIF, Savannah River Site & $\begin{array}{l}\text { TSCA incinerator, East Tennessee } \\
\text { Technical Park }\end{array}$ & WERF, INEEL \\
\hline $\mathrm{Hg}, 40 \mu \mathrm{g} / \mathrm{dscm}$ & $\begin{array}{l}\text { No. Trial bum Hg measurements } \\
\text { showed poor mass balance closure } \\
\text { and indicated inefficient Hg removal } \\
\text { in the APCS. While some Hg } \\
\text { removal is possible in the wet } \\
\text { scrubber APCS, additional Hg } \\
\text { removal, feed limits, or both will } \\
\text { likely be necessary. }\end{array}$ & $\begin{array}{l}\text { No. Hg emissions have not been } \\
\text { measured, and no mercury control is } \\
\text { assumed in current operation. While } \\
\text { some Hg removal is possible in the } \\
\text { wet scrubber APCS, additional } \mathrm{Hg} \\
\text { removal, feed limits, or both will } \\
\text { likely be necessary. }\end{array}$ & $\begin{array}{l}\text { No. Need } 99.6 \% \text { reduction for a Hg } \\
\text { feedrate of } 10 \mathrm{gh} \text { (which } \\
\text { corresponds to } 55 \text { ppmw for a } \\
400 \mathrm{lbs} / \mathrm{hr} \text { waste feedrate), } 1 / 2 \text { of the } \\
\text { maximum historical Hg feed limit of } \\
1,200 \mathrm{~g} / \mathrm{h} \text {. Would need offgas } \\
\text { control in addition to feed limits } \\
\text { because of heterogeneous nature of } \\
\text { waste feed. }\end{array}$ \\
\hline $\mathrm{PM}, 0.015 \mathrm{gr} / \mathrm{dscf}$ & Yes & $\begin{array}{l}\text { Yes, if no sludge fed. } \\
\text { No, when sludge is fed. }\end{array}$ & Yes \\
\hline $\mathrm{HCl} / \mathrm{Cl}_{2}, 75 \mathrm{ppmv}$ & Yes & Yes & $\begin{array}{l}\text { No. Compliance without } \\
\text { modification for } \mathrm{HCl} \text { control would } \\
\text { require a } \mathrm{Cl} \text { feedrate limit of } \\
0.2 \mathrm{lbs} / \mathrm{hr}(0.05 \mathrm{wt} \% \text { for max } \\
\text { feedrate of } 400 \mathrm{lbs} / \mathrm{hr} \text { ), which is } \\
\text { considered unfeasible for most waste } \\
\text { streams. Need }-95 \% \mathrm{HCl} / \mathrm{Cl}_{2} \\
\text { reduction to maintain the current Cl} \\
\text { feedrate limit of } 4 \mathrm{lbs} / \mathrm{hr}(1 \text { wt } \% \mathrm{Cl} \\
\text { in feed), or } 98 \% \mathrm{HCl} / \mathrm{Cl}_{2} \text { reduction } \\
\text { for a } \mathrm{Cl} \text { feedrate of } 10 \mathrm{lbs} / \mathrm{hr} \\
\text { ( } 2.5 \mathrm{wt} \% \mathrm{Cl} \text { in feed). }\end{array}$ \\
\hline $\mathrm{CO}, 100 \mathrm{ppmv}$ & Yes & Yes & Yes \\
\hline $\mathrm{HC} 10 \mathrm{ppmv}$ & $\begin{array}{l}\text { Assume yes based on efficiency } \\
\text { indicated by DRE and CO } \\
\text { measurements, but requires } \\
\text { confirmational testing. }\end{array}$ & & \\
\hline SVM, $100 \mu g / d s c m$ & Yes & No. Need to eliminate sludge waste & Yes \\
\hline $\mathrm{LVM}, 55 \mu \mathrm{g} . / \mathrm{dscm}$ & Yes & $\begin{array}{l}\text { feed and blend wastes to decrease } \\
\text { SVM and LVM feedrates to } 5-10 \% \\
\text { of existing permitted feed limits. }\end{array}$ & Yes \\
\hline CEMs compliance & Need PM and HC monitors & Need PM and $\mathrm{HC}$ monitors & Need PM and HC monitors \\
\hline \multicolumn{4}{|c|}{$\begin{array}{l}\text { a. Soelberg, N. R., P. Knecht, and B. Hass, "Air Pollution Control Status and Needs for Thermal Treatment Systems," presentation at the } \\
1997 \text { PADOE National Technical Workgroup Annual Meeting, Newport Beach, CA, November 5-7, } 1997 \text {. }\end{array}$} \\
\hline \multicolumn{4}{|c|}{ b. DOE, “Summary of DOE Incineration Capabilities,” DOE/D-10651, July 1998, Revision 1.} \\
\hline \multicolumn{4}{|c|}{$\begin{array}{l}\text { c. Soelberg, N. and A. Chambers, "Screening Matrix of Modifications to Enable the WERF Incinerator to Meet the Proposed MACT Rule," } \\
\text { intermal draft INEEL report, January } 1998 .\end{array}$} \\
\hline \multicolumn{4}{|c|}{ d. Idaho National Engineering and Environmental Laboratory, "WERF High Temperature Trial Bum Plan," DOEMD010131(-98), May 1998.} \\
\hline \multicolumn{4}{|c|}{$\begin{array}{l}\text { e. Knecht, M. A. and S. J. Priebe, "Proposed "MACT" Rule for Hazardous Waste Incinerators and Potential Impacts on DOE MLLW } \\
\text { Incinerators," presentation at INEEL on July 16,1997. }\end{array}$} \\
\hline \multicolumn{4}{|c|}{ f. Radian International, “Trial Bum Report, Consolidated Incinerator Facility, Savannah River Site,” July 16, 1997.} \\
\hline \multicolumn{4}{|c|}{$\begin{array}{l}\text { g. IT Corporation and Focus Environmental, "RCRA Trial Bum Report for a Dual Purpose/TSCA Incinerator at the U.S. Department of } \\
\text { Energy's K-25 Facility in Oak Ridge, Tennessee," August 31, } 1989 .\end{array}$} \\
\hline \multicolumn{4}{|c|}{ h. Stack gas concentration limits are adjusted to $7 \% \mathrm{O}_{2}$. } \\
\hline
\end{tabular}

implementation of some upgrades for specific pollutants may allow expansion of some existing WAC limits, resulting in higher throughputs and possible acceptance of wastes not currently accepted.

The MACT rule may require THC and PM monitoring. None of the three facilities have these monitors, although the TSCA incinerator has been a host test facility for $\mathrm{Hg}$ and PM monitors and will continue to test PM monitors. The MACT rule will also require some upgrades of the automatic waste feed cutoff to be interactive with the required CEMs and possibly with added continuous monitors of 
process units (such as temperature, pressure, and flow indicators) used to show that the process is operating within acceptable limits.

In addition to the CEM and automatic waste feed cutoff upgrades summarized above, compliance with the proposed hydrocarbon limit should be confirmed for all three facilities. While none of these facilities presently have online total hydrocarbons (THC) monitors, the combustion efficiency demonstrated during trial burns and continuous $\mathrm{CO}$ monitoring indicates that each facility would likely meet the THC limit. Therefore, no upgrades are expected to be neessary to comply with this limit.

\subsubsection{Consolidated Incinerator Facility}

The CIF is available for treating characteristic organic liquid (benzene) wastes $(2,100 \mathrm{~m} 3)$ from the Defense Waste Processing Facility in addition to large amounts of SRS mixed waste and LLW. Changing plans for the Defense Waste Processing Facility may eliminate the need to treat this waste stream. Besides the benzene waste stream, $5,500 \mathrm{~m}^{3}$ of other mixed waste and $103,000 \mathrm{~m}^{3}$ of LLW is destined for CIF incineration.

The CIF offgas system consists of a quick quench, Hydrosonic single-stage scrubber, cyclone separator, demister, reheater, and HEPA filters. The scrubber liquid is recirculated, and a portion is also discharged to the quench system to make up quench water evaporated in the quench unit. Clean makeup water is added to the scrubber to replace scrub solution discharged to the quench system. Thus, the "dirtiest" water is contacted with the "dirtiest" gas, and the "cleanest" water is contacted with the "cleanest" gas. The quench water is recirculated until total dissolved solids levels approach $10 \%$ and total suspended solids levels approach 3\% (both permitted limits). As these solids levels approach the permitted limits, the spent quench water is sent to one of two blowdown tanks for subsequent stabilization for disposal. The scrubber liquid is subject to the same permitted total dissolved solids and total suspended solids limits, but is maintained below these limits by maintaining the necessary ratio of recycle and discharge to the quench system. The CIF APCS contains redundant $\mathrm{CO}$ and $\mathrm{O}_{2} \mathrm{CEMs}$. Total hydrocarbons CEM measurements have not been made, but the CIF will likely meet the THC limit.

Based on the 1997 RCRA trial burn, CIF can meet most, but not all proposed MACT standards. The CIF can meet $\mathrm{HCl} / \mathrm{Cl}_{2}, \mathrm{CO}, \mathrm{SVM}$, and LVM standards. The CIF design, which rapidly cools the offgas to well below $400^{\circ} \mathrm{F}$, is considered MACT for producing low D/F emissions, but the $1997 \mathrm{RCRA}$ trial burn emissions ranged from $0.65-4.2 \mathrm{ng} / \mathrm{dscm}$ TEQ at $7 \%$ oxygen. Thus, the proposed MACT D/F emissions limits were not met.

The majority of $\mathrm{D} / \mathrm{Fs}$ may be formed in the offgas in the quench chamber, where $\mathrm{D} / \mathrm{F}$ precursors and PM from both the secondary combustion chamber (SCC) offgas and the recycled quench solution enter the quench chamber. While D/F precursors and PM in the offgas pass rapidly through the D/F forming window only once (as the gas is cooled from the hot SCC outlet temperature to the gas saturation temperature), D/F precursors and PM concentrated in the quench solution can pass through the D/F forming temperature window many times as the quench solution laden with these materials is recycled to the quench chamber. The overall residence time of $\mathrm{PM}$ and $\mathrm{D} / \mathrm{F}$ precursors in the $\mathrm{D} / \mathrm{F}$ forming temperature window can then be many times longer than the residence time calculated when considering only PM and D/F precursors in the SCC offgas alone.

The CIF trial burn results also indicate that the MACT Hg emissions limits would be exceeded, although mass balance closure was poor and the measurement data quality is suspect. No Hg removal from the offgas is assumed in current operations. The CIF is currently being researched to better understand how $\mathrm{Hg}$ behaves in the CIF system. 


\subsubsection{Toxic Substances Control Act Incinerator}

The TSCA Incinerator offgas system includes a quick wet quench, venturi scrubber, demister, packed bed scrubbers, and ionizing wet scrubbers. The quick wet quench and wet scrubber system are considered MACT for achieving D/F emissions low enough to meet the proposed rule. Prior trial burn results show that $\mathrm{D} / \mathrm{F}$ emissions are approximately one-tenth of the proposed limit. A trial burn scheduled in 1999 is expected to confirm that D/F levels can meet the MACT limit.

The $\mathrm{CO}$ is continuously monitored and measured $\mathrm{CO}$ levels will also meet MACT standards. While data are not available for $\mathrm{THC}$ and $\mathrm{Cl}_{2}$ emissions, compliance to $\mathrm{HCl} / \mathrm{Cl}_{2}$ and hydrocarbon limits is also expected. This assumption should be confirmed with testing.

Particulate matter control efficiency for the TSCA incinerator to meet the MACT PM emissions standard is less certain than for the WERF and CIF, which are HEPA-filtered. Compliance with the MACT PM limit will require either the elimination of sludge treatment or an upgrade, such as installation of an offgas reheater and HEPA filters. At present, the facility plans to eliminate sludge feeding rather than make capital equipment modifications.

Volatile metal control are directly related to PM control; so, TSCA incinerator compliance with the proposed MACT standards for LVM and SVM is also tenuous. Without equipment upgrades, LVM and SVM feedrate limits would need to be decreased in the range of 5-10\% of the currently permitted feed limit to achieve the MACT-required emissions levels for these constituents. The TSCA incinerator currently operates at only about $20 \%$ of the permitted feed limit for these constituents; so, a reduction to $5-10 \%$ of the permitted limit would mean a reduction to one-fourth to one-half of current feed levels. At present, the facility plans to comply with the SVM and LVM limits by controlling the feedrate of these metals.

Wet scrubbing for $\mathrm{Hg}$ control is highly variable. The $\mathrm{Hg}$ scrubbing efficiency of the TSCA incinerator wet scrubber system has not been accurately measured. The EPA test data used to develop the proposed MACT rule indicate that wet scrubber $\mathrm{Hg}$ control efficiency varies from around $10 \%$ to over $90 \%$. Because of the rapid quench that could tend to "freeze" $\mathrm{Hg}$ in the difficult-to-scrub elemental $\mathrm{Hg}$ form rather than the easy-to-scrub $\mathrm{HgCl}_{2}$ form, $\mathrm{Hg}$ control efficiency in the TSCA incinerator wet scrubber may be low. The TSCA incinerator will require some combination of stringent $\mathrm{Hg}$ feed control, operating modifications, and installation of $\mathrm{Hg}$ emissions control equipment to meet the MACT $\mathrm{Hg}$ limit. Compliance by feed control alone would require an average $\mathrm{Hg}$ feed concentration limit as low as 0.2-2 ppmw, depending on the achievable wet scrubber $\mathrm{Hg}$ control efficiency. This may be achieved by eliminating heterogeneous solid wastes (which may be impossible to accurately characterize at such low $\mathrm{Hg}$ levels using currently available technologies). The remaining homogeneous waste may be able to be characterized to this level, allowing compliance to be achieved by feed limits and wet scrubbing.

\subsubsection{Waste Experimental Reduction Facility}

The WERF offgas system consists of an air-to-air heat exchanger, controlled introduction of dilution air for further cooling, and a baghouse followed by $\mathrm{HEPA}$ filters. WERF has $\mathrm{HCl}, \mathrm{CO}, \mathrm{O}_{2}$, and total combustibles CEMs. The HEPA filtered offgas currently meets the MACT PM, LVM, SVM, CO, and emissions standards. Compliance with the hydrocarbon standard is assumed until testing can confirm this. The WERF offgas system does not include control equipment specifically for $\mathrm{D} / \mathrm{F}, \mathrm{Hg}$, or $\mathrm{HCl}^{-\mathrm{Cl}_{2}}$ control, although tests have shown limited $\mathrm{HCl}$ scrubbing in the baghouse.

Dioxin/furan levels are expected to meet existing risk-based permit limit, but the not the MACT rule limit. The SCC offgas is cooled from the SCC temperature of around $1,900^{\circ} \mathrm{F}$ to the baghouse inlet 
temperature of around $395^{\circ} \mathrm{F}$ relatively slowly. Ambient-temperature dilution air is blended with the hot offgas to cool the mixture to around $1,250^{\circ} \mathrm{F}$. A gas to air shell and tube heat exchanger further cools the gas to around $625^{\circ} \mathrm{F}$, and additional ambient temperature air is added to further cool the gas to the baghouse inlet temperature. This configuration allows some formation of $D / F$ s from the trace amounts of precursors in the offgas.

In recent years, various modifications have been conducted to improve overall incinerator performance, improve organic destruction and removal efficiency, and lower $\mathrm{D} / \mathrm{F}$ emissions. While these modifications have not resulted in D/F levels as low as the proposed MACT rule limit, they have improved DRE and lowered D/F levels enough to meet existing permit requirements. WERF plans to install new burners and improve combustion air control, which should further improve system combustion performance. These already-planned improvements are incorporated in all of the WERF MACT compliance options. The costs for these improvements are not included in the MACT upgrade costs.

The WERF does not presently meet the MACT rule $\mathrm{HCl} / \mathrm{Cl}_{2}$ limit at the current permitted $\mathrm{Cl}$ feed limit of $4 \mathrm{lbs} / \mathrm{hr}$, which was established to ensure that no more than $4 \mathrm{lbs} / \mathrm{hr} \mathrm{HCl}$ exited the incinerator in the offgas, assuming zero $\mathrm{HCl}$ system removal efficiency. The $4 \mathrm{lbs} / \mathrm{hr} \mathrm{HCl}$ emission limit equates to about 1,000 ppmv $\mathrm{HCl}$ in the offgas $\left(\right.$ at $\left.7 \% \mathrm{O}_{2}\right)$. The feedrate of $\mathrm{Cl}$ is administratively controlled at an even lower feedrate of $2 \mathrm{lbs} / \mathrm{hr}$ to ensure compliance with the $4 \mathrm{lbs} / \mathrm{hr} \mathrm{HCl}$ emission limit. Even at the administrative $2 \mathrm{lbs} / \mathrm{hr} \mathrm{Cl}$ feed limit, the $4 \mathrm{lbs} / \mathrm{hr} \mathrm{HCl}$ emission limit has proven difficult to always meet because of periodic releases of $\mathrm{HCl}$ as chlorinated organics in the ash pile combust. The even lower concentration-based MACT limit for $\mathrm{HCl} / \mathrm{Cl}_{2}$ is not achievable using feed control alone. Equipment upgrades or operating modifications, used alone or in combination with feed limits, will be needed to lower total $\mathrm{HCl} / \mathrm{Cl}_{2}$ emission levels by at least $95 \%$.

Without any $\mathrm{Hg}$ control in the offgas system, the Hg concentration in the WERF feed would need to be reliably controlled to less than $\sim 0.2 \mathrm{ppmw}$. Such low $\mathrm{Hg}$ concentrations are difficult to reliably characterize, especially for heterogeneous solid wastes. Compliance with the MACT Hg standard would be very difficult to meet with confidence for many waste streams.

For a more complete description of CIF and TSCA Incinerator and the potential MACT upgrade options, see MACT System Integration Study Draft Status Report. ${ }^{7}$

\subsection{Current WERF Waste Acceptance Criteria}

The WERF WAC is used to identify waste that is acceptable or unacceptable for treatment. The WAC specifies acceptable limits for mass, radionuclides, liquid viscosity, water content, heating value, and specific chemical content. The WERF WAC is based upon applicable regulations, DOE orders, and the receiving facility specific requirements. The WERF accepts LLW and MLLW for treatment by incineration. Most limits for specific chemical compounds or elements pertain to MLLW. Because of the current lack of chlorine-removal technology in the WERF APCS, the administrative limit for chlorine is generally one-half the WERF WAC for chlorine. The current WAC limits that administratively control the amount of chlorine or mercury in the waste are listed in Table 2-3. 


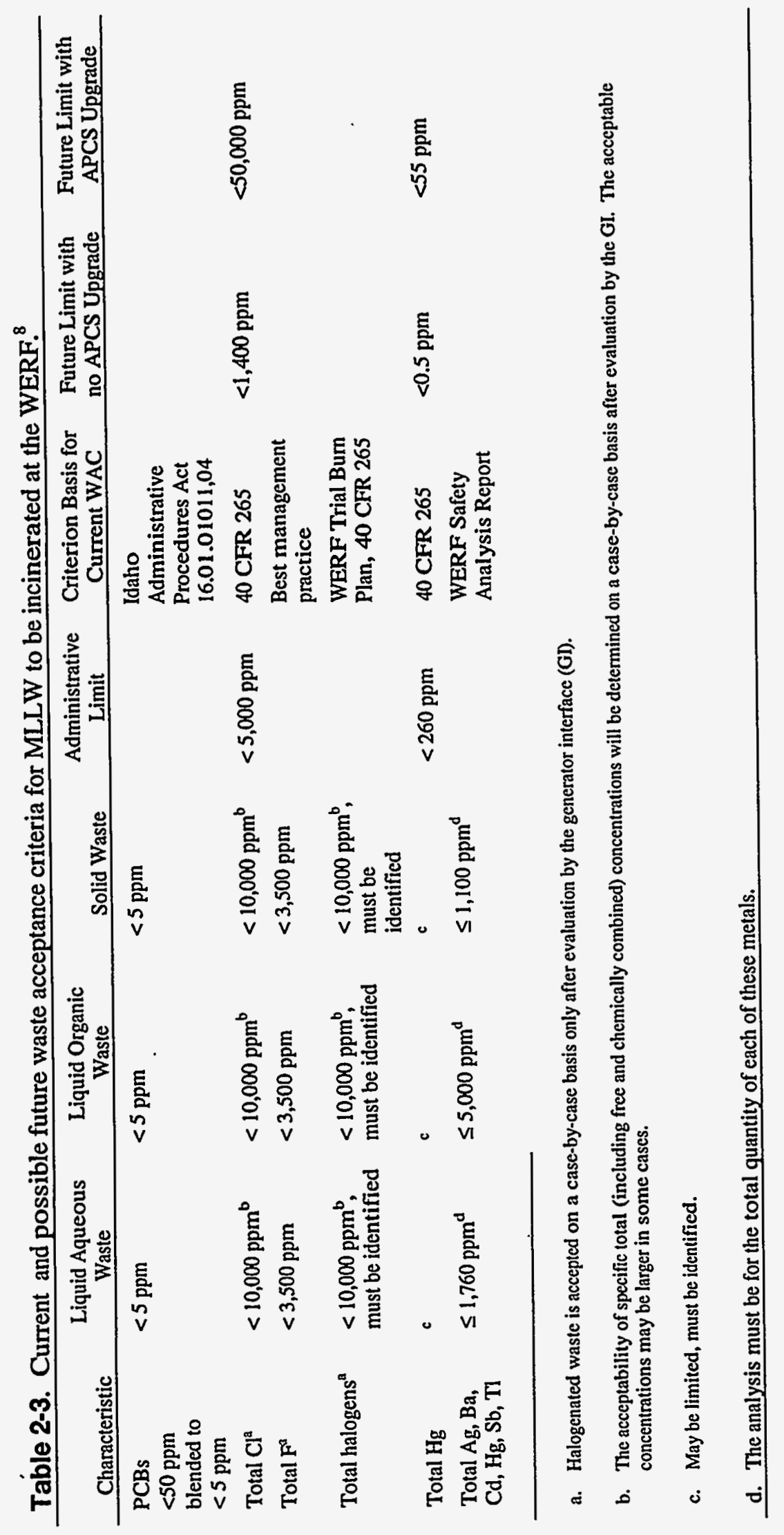




\subsubsection{MACT Emission Limits Versus WERF's Current Emission Levels}

The proposed limits and WERF emissions data for comparison are summarized in Table 2-4. In order to meet the proposed MACT ruling the data clearly indicates that the emission of HAPs of concern for the WERF include $\mathrm{HCl} / \mathrm{Cl}_{2}, \mathrm{Hg}, \mathrm{D} / \mathrm{F}$, and possibly, total hydrocarbons (THC).

2.3.1.1 Hydrogen Chloride/Chlorine Gas Emissions Control. Much of the MLLW within the DOE complex contains polyvinyl chloride (PVC) or chlorinated chemical compounds. PVC is a common plastic used in personal protective equipment such as shoe covers, gloves, anti-contamination clothing, and bubble hoods. In addition, there are chlorinated chemical compounds that were commonly used as solvents and degreasers in the DOE complex.

Using the proposed MACT limit of $75 \mathrm{ppmv}$ in the offgas, without $\mathrm{HCl}_{2} \mathrm{Cl}_{2}$ emission control, the WERF chlorine feed limit concentration is $1,400 \mathrm{ppmw}$ in the feed when treating $400 \mathrm{lbs} / \mathrm{hr}$ of waste. This represents $0.6 \mathrm{lbs} / \mathrm{hr}$ of chlorine to the incinerator. The current chlorine waste feed limit is adminstratively controlled at $4 \mathrm{lbs} / \mathrm{hr}$ to ensure that $\mathrm{HCl}$ release is maintained at or below $4 \mathrm{lbs} / \mathrm{hr}$. Even at this chlorine feedrate, much effort is required to segregate the chlorine-containing waste in the MLLW to avoid violating the permitted value.

Since chlorinated compounds represent a significant portion of the DOE MLLW inventory, waste segregation for chlorine feed control is not considered practical. Therefore, this study evaluated $\mathrm{HCl}$ control technologies that could be retrofitted into the WERF incinerator/offgas treatment system. At incinerator conditions, the formation of $\mathrm{HCl}$ is thermodynamically favored over that of chlorine gas. ${ }^{9}$ Chlorine gas formation is thermodynamically favored at lower temperatures, but kinetic limitations prevent a significant shift in concentrations generated in the incinerator. As a result, almost all chlorine in the APCS is in the form of $\mathrm{HCl}$. For this reason, $\mathrm{HCl}$ control in the APCS is considered equivalent to total chlorine control in this report.

2.3.1.2 Mercury Emissions Control. The current Hg emission limit at WERF, as proposed in the RCRA permit application and listed above, is approximately 160 times the MACT proposed emission level. This limit is based upon air dispersion modeling and health risk assessments.

Assuming all the $\mathrm{Hg}$ in the waste feed volatilizes, without $\mathrm{Hg}$ emission control, the MACT proposed limit translates to a waste feed $\mathrm{Hg}$ concentration of $0.5 \mathrm{ppmw}$ when feeding $400 \mathrm{lbs} / \mathrm{hr}$ of waste to the WERF incinerator. Blending mercury-contaminated waste to this concentration or reducing the waste feed rate to meet the proposed MACT emission limit is not considered to be a reasonable solution. Therefore, technologies to remove Hg from the WERF offgas were investigated.

\subsubsection{Dioxin/Furan Emissions Control. Dioxins/furans are products of incomplete} combustion (PICs) common within incineration processes. Baseline emissions data from WERF indicate that the D/F emissions rate is 20 to 200 times greater than the proposed limit. Unfortunately, the science associated with the formation of various $\mathrm{D} / \mathrm{Fs}$ is highly complicated and partially dependent on the incinerator system design. The technologies and incinerator operating conditions for controlling their formation are primarily based upon what has provided relatively low D/F concentrations in similar applications. Basically, there are incinerator offgas temperature ranges and gas residence times that are favorable for $\mathrm{D} / \mathrm{F}$ formation. 
Table 2-4. Comparison of WERF emissions data to MACT limits.

\begin{tabular}{|c|c|c|c|}
\hline & HAPs or Surrogates & $\mathrm{MACT}^{\mathrm{a}, \mathrm{b}}$ & WERF $^{a}$ \\
\hline \multicolumn{2}{|c|}{ Dioxin/Furan, ng TEQ/dscm } & 0.20 or 0.40 and $\leq 400^{\circ} \mathrm{F}$ & $\begin{array}{l}5.88^{c} \\
4.7^{d} \\
42.7^{e}\end{array}$ \\
\hline \multicolumn{2}{|c|}{ Mercury, $\mu \mathrm{g} / \mathrm{dscm}$} & 40 & $11,834^{\mathrm{f}}$ \\
\hline \multicolumn{2}{|c|}{ Particular matter (gr/dscf) } & 0.015 & $\begin{array}{l}0.007^{d} \\
0.003^{e}\end{array}$ \\
\hline \multicolumn{2}{|c|}{ Hydrochloric acid/chlorine, ppmv } & $75^{\mathrm{e}}$ & $\begin{array}{l}804^{d} \\
522^{e}\end{array}$ \\
\hline \multicolumn{2}{|c|}{ Carbon monoxide, ppmv } & 100 & $0.6-3.6$ \\
\hline \multicolumn{2}{|c|}{ Total hydrocarbons , ppmv } & 10 & $6^{8}$ \\
\hline \multicolumn{2}{|c|}{ Semi-volatile metals (Cd and $\mathrm{Pb}), \mu \mathrm{g} / \mathrm{dscm}$} & 100 & $<3.5^{\mathrm{e}}$ \\
\hline \multicolumn{2}{|c|}{$\begin{array}{l}\text { Low-volatility metals (As, Be, and } \mathrm{Cr} \text { ), } \\
\mu \mathrm{g} / \mathrm{dscm}\end{array}$} & 55 & $10.8^{\mathrm{e}}$ \\
\hline a. & \multicolumn{3}{|c|}{ All values are corrected to $7 \% \mathrm{O}_{2}$} \\
\hline b. & \multicolumn{3}{|c|}{ The limit for existing incinerators may be higher in the final rule. } \\
\hline c. & \multicolumn{3}{|c|}{ February 1998 mini-burn (numerical average of $1.9,5.2,4.5,1.8,16$ ). } \\
\hline d. & \multicolumn{3}{|c|}{ Low Temperature Trial Burn May 1997.} \\
\hline e. & \multicolumn{3}{|l|}{ High Temperature Trial Bum July 1997.} \\
\hline f. & \multicolumn{3}{|l|}{ Proposed RCRA Part B Permit Application. } \\
\hline g. & \multicolumn{3}{|l|}{ Estimated from WERF trial bum data. ${ }^{1}$} \\
\hline
\end{tabular}

The hazardous waste incinerator industry approach to $\mathrm{D} / \mathrm{F}$ control includes increasing the efficiency of the combustion process and minimizing the time a given offgas stream resides in a temperature range of $480^{\circ} \mathrm{F}-930^{\circ} \mathrm{F}$. Although improved waste feed control may also reduce $\mathrm{D} / \mathrm{F}$ formation, it is not a satisfactory option until definitive $\mathrm{D} / \mathrm{F}$ formation mechanisms and chemical compounds that contribute to the formation of these PICs are identified. This investigation identified offgas treatment processes that are used to minimize $\mathrm{D} / \mathrm{F}$ formation, and those that are designed to remove them from the offgas should they be generated. 


\subsection{Feasibility of Upgrading WERF to Treat PCBs}

Much of the waste targeted for incineration (including $60,000 \mathrm{~m}^{3}$ from the TSCA incinerator) contains polychlorinated biphenyls (PCBs). The question has been raised concerning the feasibility of modifying WERF equipment or operational controls to make the system suitable to treat waste containing PCBs regulated under the TSCA. Storing and treating PCB-contaminated waste requires approval of the EPA. WERF can and has treated non-TSCA regulated waste with PCB concentrations less than $50 \mathrm{ppm}$.

\subsubsection{Environmental Compliance Issues}

When the PCBs to be treated are mixed with hazardous waste, a TSCA permit application can frequently be combined and submitted with a RCRA Part B permit application. The additional information required by TSCA is not extensive. The most significant difference is that the TSCA approval process would require a third test be added (in addition to the low- and high-temperature tests) to the performance test (trial burn) to establish PCB waste burning limits. Personnel from the appropriate state or federal regulatory agencies review the permit application as it applies to both RCRA and TSCA requirements (TSCA is regulated by the EPA directly, not by the State of Idaho).

If a TSCA permit is desired, considerable communication with the permitting agencies will be necessary to negotiate a timely approval that coincides with the MACT compliance schedule for RCRA permit modification and construction start. The permitting agency is only obligated to comply with the streamlined RCRA permit modification requirements to accommodate timely MACT compliance upgrades; modifications associated with TSCA requirements are outside the intent of the streamlining process. If necessary, with regulator approval, a TSCA permit approval may be delayed beyond construction start, because TSCA regulations allow this.

Modifications to accommodate PCB-contaminated waste may also include upgrades to waste storage facilities in addition to the incinerator system. TSCA regulations have unique spill containment and labeling provisions for storage areas. Since PCB incineration was not analyzed in the Site-wide Environmental Impact Statement, more extensive National Environmental Policy Act documentation will likely be required. This documentation may be a supplement to the Environmental Impact Statement (determined by a supplement analysis) or a new Environmental Impact Statement or Environmental Assessment.

\subsubsection{Technical Issues}

The necessary PCB DRE of at least $99.9999 \%$ (6-9s) may not be achievable with the current incinerator design and operation. The following technical issues are relevant when determining the feasibility of PCB incineration at WERF.

2.4.2.1 Vortex Burners in PCC and SCC. Replacing both primary combustion chamber (PCC) and SCC burners with vortex burners could reduce the need for design changes to the SCC to meet 6-9s destruction and removal efficiency (DRE). A properly operated vortex burner should achieve 6-9s DRE on its own merits. The Los Alamos incinerator, which is very similar in design to WERF except that it uses vortex burners, has demonstrated 6-9s DRE for PCBs.

2.4.2.2 SCC Residence Time and Excess Oxygen. Incinerators burning TSCA-regulated waste are required to meet one of the following sets of conditions:

1. A residence time in the $\mathrm{SCC}$ of 2 seconds at a temperature of $\geq 1200^{\circ} \mathrm{C}\left(2192^{\circ} \mathrm{F}\right)$ and $\geq 3 \% \mathrm{O}_{2}$ in the exhaust 
2. A residence time in the $\mathrm{SCC}$ of 1.5 seconds at a temperature of $\geq 1600^{\circ} \mathrm{C}\left(2912^{\circ} \mathrm{F}\right)$ and $\geq 2 \% \mathrm{O}_{2}$ in the exhaust.

Attaining a SCC temperature of about $2200^{\circ} \mathrm{F}$ in the WERF incinerator, as required by condition set no. 1, appears feasible if the existing PCC and SCC auxiliary burners are replaced with vortex burners. In addition, establishment of a higher PCC operating temperature limit than is currently allowed by the existing RCRA Part B pernit application may be required. Operating the $S C C$ at about $2200^{\circ} \mathrm{F}$ will also increase maintenance costs by a limited amount.

Meeting the requirements of condition set no. 2 is not feasible for the WERF incinerator. Obtaining an SCC temperature of over $2900^{\circ} \mathrm{F}$ cannot be done without costly modifications (e.g., replacing SCC structural components), significantly increased maintenance costs (e.g., frequent refractory replacement), and potential safety concerns (e.g., possible use of oxygen-enriched combustion air).

Given the preceding requirements and limitations, it is appropriate to consider condition set no. 1 in association with a potential TSCA permit application for the WERF incinerator. As previously described, achieving $2200^{\circ} \mathrm{F}$ in the SCC appears feasible. However, evaluation of the incinerator potential to meet the 2-second residence time requirement requires familiarity with how process conditions can change in the incinerator during a waste box feed cycle.

The SCC has a fixed volume of $310 \mathrm{ft}^{3}$. The flowrate of process gas through the SCC that corresponds to a 2-second residence time can be calculated as follows:

$$
\text { Flowrate }(a c f m)=\left(\frac{310 c f}{2 s}\right) *\left(\frac{60 s}{m}\right)=9,300 a c f m .
$$

This equation indicates that an SCC gas flowrate less than 9,300 acfm provides a gas residence time greater than 2 seconds. If the gas flow is higher, then the gas residence time is less than $\mathbf{2}$ seconds.

The contributors to the value of the SCC gas flowrate are nitrogen, oxygen, and combustion gases. Air enters the system as combustion air and as in-leakage (since the incinerator is operated under a slight vacuum). All of the nitrogen and the excess oxygen that is not consumed during combustion become SCC gas flow. Combustion gases are produced by combustion of both waste and auxiliary fuel. During normal waste feeding operations, combustion air is added only through the SCC auxiliary burner and at a location between the PCC and SCC. This allows the system to operate, as designed, with the PCC having a low gas flowrate so that particle entrainment is minimized while maintaining a sub-stoichiometric amount of oxygen. The flameport air introduced between the PCC and SCC is fed at a rate that ensures that the SCC operates with an excess of oxygen. An excess of oxygen in the SCC is needed for ensurance of complete destruction of hazardous matter.

A box feed cycle consists of the following steps:

1. A box is automatically conveyed to the vertical box charging system hatch (which is closed).

2. The operator verifies that process conditions, including the oxygen content of the SCC, are satisfactory for feeding waste.

3. When initiated by the operator, the hatch opens, a box is conveyed into the box charging system and falls onto the top charging door (which is closed), and the hatch closes. 
4. Immediately after the hatch is closed, the flameport air flow increases from minimum to maximum flow.

5. The ash ram is stroked, or moved from its home position outside the PCC hearth area across the bottom of the hearth to a pre-set location along the hearth (thus, cleaning the hearth prior to feeding a box and pushing into the ash pile the unburned material from the last box fed), and then, is returned home to complete the stroke.

6. The two charging doors open, and the waste box falls onto the PCC hearth and begins to burn.

7. The cycle is repeated at the appropriate time.

The gas flowrate and associated gas residence time and oxygen content of gas coming out of the SCC are monitored and as shown in Figures 2-1 and 2-2, vary significantly over the course of a box feed cycle. Significant feed cycle events and process responses to the events are described in the following discussion.

When the flameport air flowrate is at its minimum value, the SCC gas residence time is about 3.5 seconds. Concurrently, the SCC outlet gas oxygen concentration is increasing as it recovers from the effects of the last waste box feed. When the hatch is opened, the flameport air flowrate begins to increase to its maximum rate. The gas flowrate increases by over $4,000 \mathrm{acfm}$ when the hatch, which is equipped with seals, is opened, decreases by about 3,000 acfm when the hatch is closed, and then increases again about 4,000 acfm due to the increasing flameport air flowrate. With the flameport air flowrate at its maximum, the residence time drops to just less than 2 seconds, and the oxygen content increases.

Just after 0700 , the oxygen concentration begins to decrease rapidly due to prompt release of combustible matter from the ash pile as a result of an ash ram stroke. The gas flowrate increases rapidly while the rate of oxygen content decrease slows. The gas residence time also noticeably decreases below 2 seconds. These responses are due to the waste box bursting into flames in the PCC. After a short time, the rate of burning of the remaining contents of the box decreases, and in turn, the gas flowrate decreases and oxygen content increases.

After a pre-set time, the flameport air flowrate automatically returns to its minimum value. Prior to that time, the oxygen concentration increases at a moderate rate. Once the flameport air flowrate is decreased, the oxygen recovers at a slower rate because oxygen is still being consumed by combustible material released from the ash pile.

Higher temperatures are desirable and are achieved with proper heat content of the waste, feed rate of the waste boxes, and operation of the SCC auxiliary burner as needed for supplementary heat. Operation of the incinerator within an envelope of conditions that ensures proper destruction of hazardous waste requires proper control of several interdependent process variables. If one desired to increase the residence time by decreasing secondary gas flowrate, one might decrease the size (and thus combustible content) of the waste box. However, in doing so, the heat content would be decreased and the temperature would fall unless additional heat was added, possibly by the SCC auxiliary burner. But turning up the auxiliary burner would add to the gas flowrate, thus negatively impacting residence time. Another way of increasing the heat input to maintain temperatures would be to add smaller boxes at a faster rate. However, there would need to be enough air introduced to maintain oxygen content. Again, adding additional air would increase the gas flowrate and decrease residence time. 


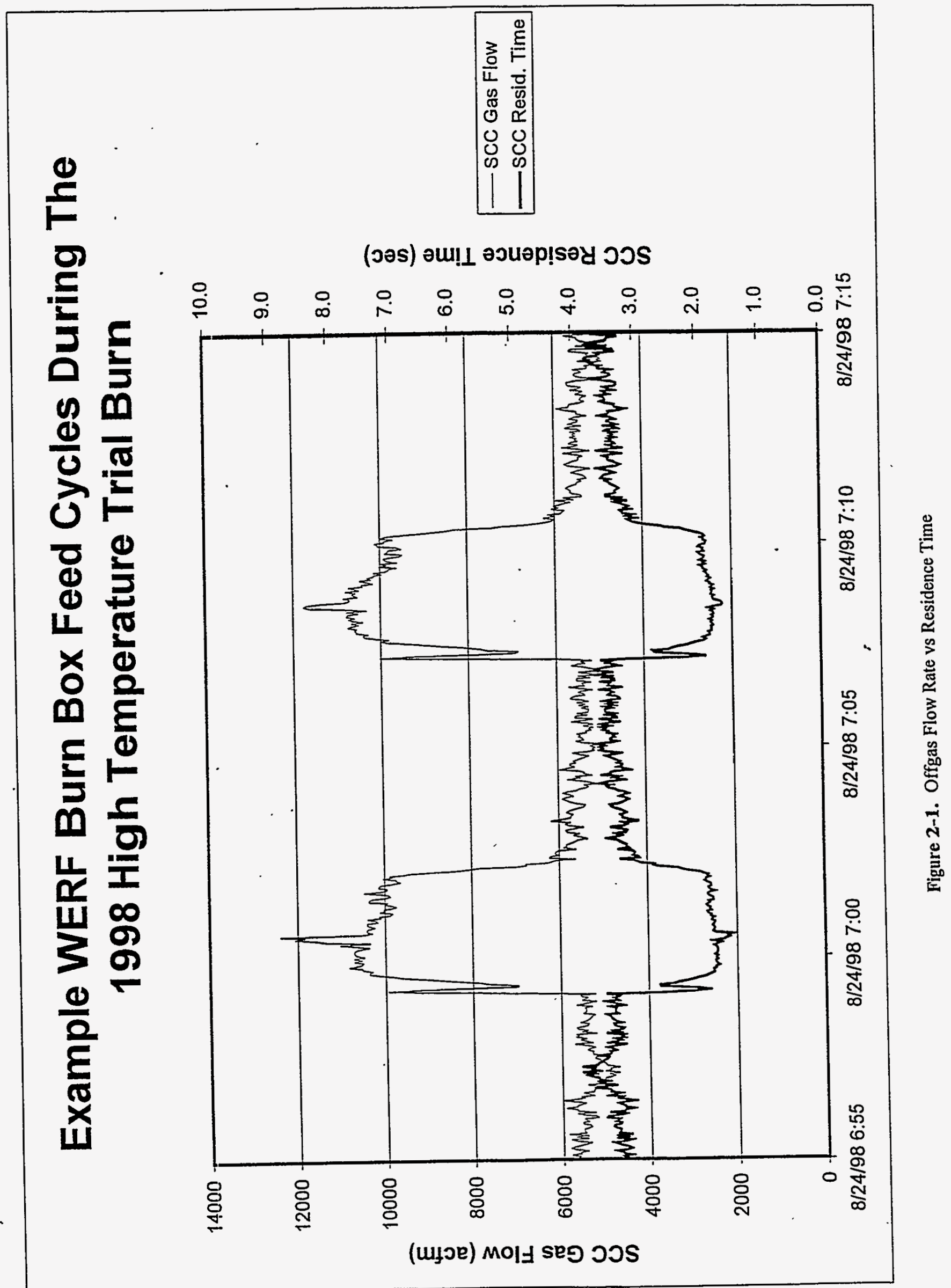




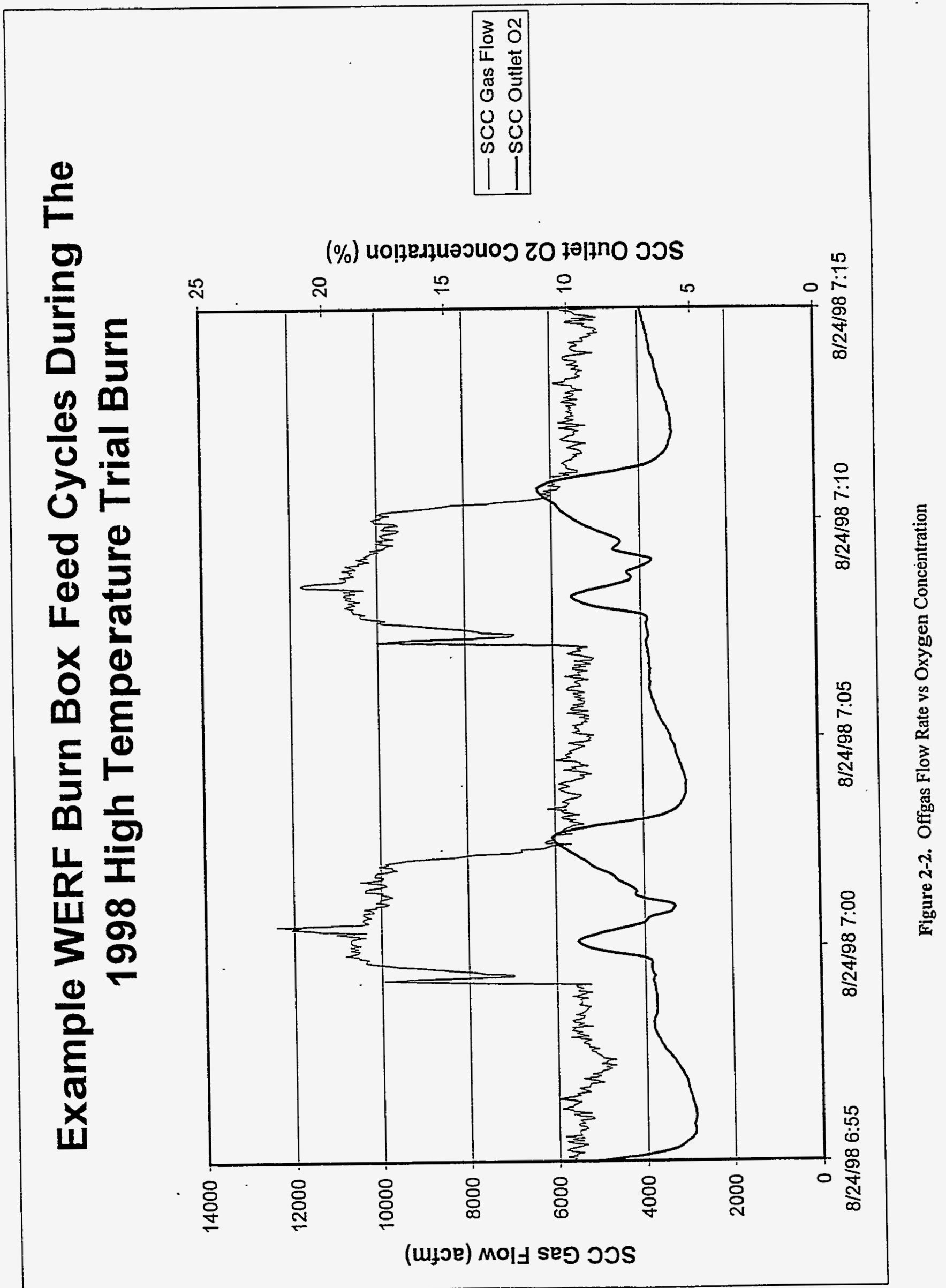


The preceding discussion should not be interpreted to conclude that processing waste while maintaining a residence tirne of at least 2 seconds is not feasible. However, the current typical waste box configuration would change and incinerator process control would require optimization based on the new configuration. Therefore, a separate trial burn test at different conditions would be proposed for TSCA waste. Decreasing the overall waste feed rate would likely be required, as well. An average residence time of over 2 seconds is easily achievable and as can be concluded from the figures which correspond to a nearly $400 \mathrm{lbs} / \mathrm{hr}$ waste feed rate, is achieved during current operations.

Permitting agencies have allowed residence time to be calculated from a combustion gas velocity indicator that was recorded on an hourly rolling average basis. However, this determination is unique to the facility, and regulators may not allow this method for a batch waste feed process, such as WERF.

\subsubsection{Other Considerations}

An appropriately designed carbon bed system, similar to that currently included in MACT upgrade options, can be expected to efficiently control trace organics including PCBs. TSCA regulations require a water scrubber for $\mathrm{HCl}$ removal in a PCB incinerator. However, the regulations allow for an alternative method of $\mathrm{HCl}$ control with approval from the regulator; equivalent gas cleaning equipment with similar $\mathrm{HCl}$ removal capabilities should be acceptable. Continuous monitoring of $\mathrm{CO}$ and $\mathrm{O}_{2}$ is required whenever incinerating PCBs. Periodic monitoring of $\mathrm{CO}_{2}$ is required, as well, at a frequency set by the regulator.

A. 2 ppm PCB residue limit is normally required in TSCA permits as an indicator of incinerator performance. This means that ash residues must contain less than 2 ppm PCBs; this may be difficult for the WERF incinerator to achieve when burning solid waste with high PCB levels.

\subsubsection{Recommendations}

After a careful review of the technical and regulatory issues surrounding the treatment of TSCA waste, it is recommended that the WERF MACT upgrade project make an application to include the treatment of TSCA waste. This activity can be conducted concurrently with the WERF RCRA application at very little additional expense. The scope of the required changes can be developed further, and a separate cost estimate can be prepared. The technical material for the permit application will already be prepared as part of the RCRA application modification so the major costs will be in meeting the National Environmental Policy Act requirements and the additional trial burn for PCBs. A rough order of magnitude cost for these activities is $\$ 400 \mathrm{~K}$. 


\section{EQUIPMENT STATUS FOR THE WERF INCINERATION PROCESS}

This section of the report evaluates the life expectancy of the existing incinerator and APCS at WERF to ensure that upgrading the facility to comply with MACT requirements is economically justifiable compared to the option of entirely replacing the incineration process. Additionally, there are a number of corrective maintenance activities and process improvements that are being or should be considered in conjunction with a MACT upgrade that would optimize the incineration process. The following sections describe the process and discuss these issues in greater detail.

\subsection{Process Description}

The WERF incineration process consists primarily of two systems. They are the incinerator and APCS. Contained within the incinerator system is the PCC, SCC, burner systems, combustion air subsystems, and the bottom ash removal system. The APCS includes a number of components including the heat exchanger, baghouse, HEPA filtration unit, fans, and stack. The WERF incineration process is described in detail in the following sections.

\subsubsection{Incinerator}

The WERF incinerator is a dual-chambered (primary and secondary chamber), controlled-air incinerator, manufactured by Environmental Control Products of Charlotte, North Carolina, with a thermal capacity of $6.5 \mathrm{MBtu} / \mathrm{hr}$. Each cylindrical chamber is horizontally oriented with a length of $14 \mathrm{ft}$ and a diameter of $5 \mathrm{ft}-4$ in. Both chamber shells are constructed using 0.25 in. carbon steel and are refractory lined (Figure 3-1).

Each chamber is equipped with a \#2 fuel oil-fired burner that is used to preheat the system and maintain operating temperatures necessary for waste processing. Typical operating temperature is in the range of $1600^{\circ} \mathrm{F}$ to $2000^{\circ} \mathrm{F}$ in the $\mathrm{PCC}$ and $1800^{\circ} \mathrm{F}$ to $2100^{\circ} \mathrm{F}$ in the SCC. Each of the burners are equipped with dedicated blowers to supply combustion air. Additional combustion air can be added to the PCC through a series of underfire air ports that are located at the bottom of the incinerator near the burning feed material and bottom ash.

Solid waste is gravity fed to the PCC where it is oxidized in an oxygen-deficient environment. This substoichiometric erıvironment is maintained by controlling the air introduced into the primary chamber so that volatile materials can be driven off in a controlled manner, and particulate emissions are minimized. This feature is effective for controlling radioactive contamination since most of the radionuclides are associated with the PM.

As the volatile materials pass into the SCC, they pass a flow restriction, called the flame port region, where excess air is introduced. This restricted area is designed to provide turbulent mixing of the air and volatiles and contribute to destruction efficiency of the volatile material in the SCC.

An ash ram, located in the hearth area of the PCC, is used to move the bottom ash from the feed chute area prior to feeding another package of waste. The ash ram is also used to incrementally move the ash pile towards a discharge chute at the end of the chamber. Moving the ash this way ensures complete burn-out of the volatiles. Bottom ash is normally removed from the PCC after cooling via a discharge chute at end of the incinerator. The ash passes through a shredder to size reduce large clinkers and then falls into a drum. 


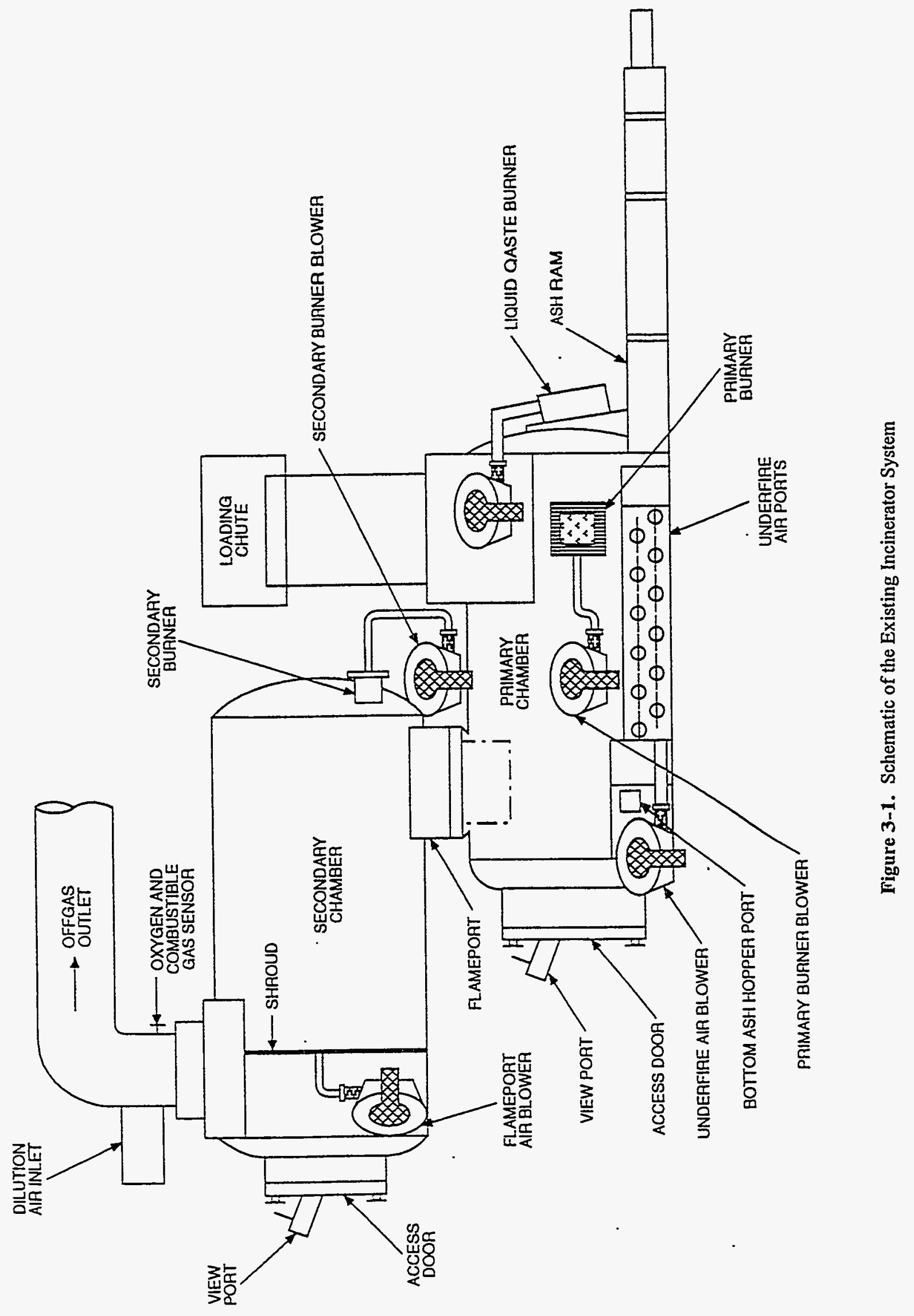




\subsubsection{Air Pollution Control System}

The incinerator APCS is a dry mechanical air-filtering system. The APCS was designed to maximize the control of particulate and metal emissions, but does not provide for the control of acid gas. As a result, $\mathrm{HCl}$ emissions are controlled by limiting the amount of chlorine in the waste and the feed rate to the incinerator (Figure 3-2).

The incinerator APCS components are also an integral part of the building (PER-609) ventilation system. Ventilation air from the building is extracted continuously into the incinerator APCS. If the incinerator is operating, the ventilation air is mixed with the incinerator offgas. If the incinerator is not operating, ventilation air is still extracted. The ventilation system is designed to prevent fugitive emissions by filtering all of the air that leaves the building and by maintaining the building pressure lower than atmospheric conditions.

A three-step process is used for cooling the combustion gas exiting the SCC prior to entering downstream APC components. In the first step, approximately 1,500 acfm of outside air at ambient temperature is mixed with the offgas just downstream of the SCC to reduce the offgas temperature to less than $1,350^{\circ} \mathrm{F}$. The offgas then passes through the air-to-air heat exchanger where its temperature is further reduced to approximately less than $650^{\circ} \mathrm{F}$. Downstream of the heat exchanger is a spark arrestor. The spark arrestor consists of a woven mesh screen constructed of stainless steel and is designed to prevent burning particulate that may have carried over into the offgas from the PCC from reaching the baghouse. After exiting the heat exchanger, approximately 3,000 acfm of building air (at $70^{\circ} \mathrm{F}$ ) is added to the offgas to further cool it to less than $400^{\circ} \mathrm{F}$ before entry into the baghouse.

The WERF baghouse system consists of two pulse-jet type baghouses. They are installed in parallel with a common outlet plenum. Each baghouse is rated to filter $8,000 \mathrm{acfm}$ (at $400^{\circ} \mathrm{F}$ ) of offgas continuously. Each is a dust-tight compartment containing 144 filter bags that provides an air-to-cloth ratio of 6:1. Fly ash is normally removed from the system via the pneumatic transfer system that is installed at the bottom of the baghouse hoppers. The transfer line carries the fly ash to a smaller collector, and the ash is dumped into a drum.

The offgas exits the baghouse and flows into a HEPA filtration unit designed to remove submicron particles that the baghouse filters cannot capture. The HEPA filtration system consists of a stainless-steel housing, a prefilter rack holding 16 prefilters, and a HEPA filter rack holding an array of 16 HEPA filters. The HEPA filters are nuclear-grade constructed using acid-resistant media and stainless steel frames. The HEPA filters have a rated minimum particulate removal efficiency of $99.97 \%$ for $0.3 \mu \mathrm{m}$ particles. Removal efficiency for smaller and larger particles will be higher.

The offgas then flows through a duct into two induced draft fans. The fans are used to maintain a negative pressure within the APCS and to transport the offgas from the incinerator through the cooling process, the baghouse, the HEPA filtration unit, and finally, through a 49-ft high stack. Operation of both fans is required when the incinerator is operating. 


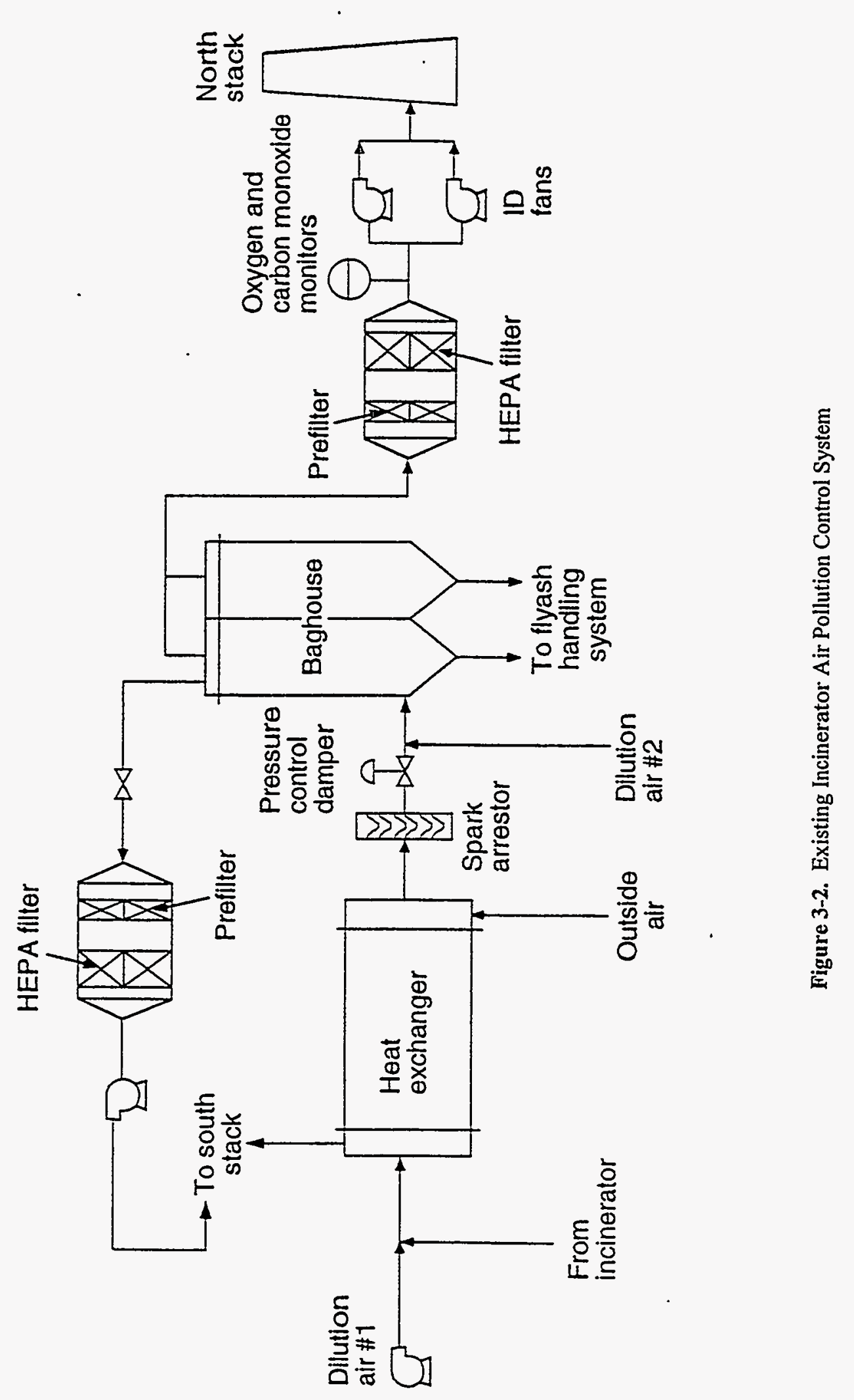




\subsubsection{Continuous Emissions Monitors}

The WERF CEMs are designed to measure the content of $\mathrm{CO}, \mathrm{HCl}$, and oxygen in the incinerator offgas after it has passed through the HEPA filtration system. Monitoring of $\mathrm{CO}$ is required per $40 \mathrm{CFR}$ $266^{10}$ The oxygen concentration in the offgas is also measured at the same location and is used to correct the measured $\mathrm{CO}$ value to an equivalent value at $7 \%$ oxygen. Because the WERF APCS does not have the ability to remove $\mathrm{HCl}$ present in the offgas, $\mathrm{HCl}$ concentrations in the offgas are also monitored to demonstrate that the WERF does not exceed EPA waste feed limit of $4 \mathrm{lbs}$ of $\mathrm{HCl}$ per hour (for incinerators not controlling $\mathrm{HCl}$ emissions).

A sample pump continuously extracts an offgas sample from downstream of the HEPA filtration system. The sample is passed through a sample-conditioning unit to prevent condensation from forming in the sample line before reaching the analyzers. The $\mathrm{CO}$ and $\mathrm{HCl}$ monitors rely on attenuation of a light beam passed through a sample to indicate the quantities of gas present in the sample. The oxygen monitor uses a zirconium oxide cell to determine the oxygen concentration of the offgas, as compared to the oxygen concentration of the ambient air.

Additionally, a radiological isokinetic sampling system continuously draws a small quantity of air from the north stack and passes it through one of two parallel filters. These filters are periodically removed and sent off-site for radiological analysis.

\subsection{Equipment Assessment}

The WERF has completed nearly 20,000 hours of incinerator operations since the facility began processing low-level radioactive waste in 1984. Since then, the WERF has undergone only one extended shutdown. The shutdown, which began in February 1991 and concluded in July 1995, was used to perform major modifications and repairs to system hardware, re-establish system configuration control, and revise operating procedures. During this time, an in-depth assessment of major incinerator system components was also conducted. The assessment included the performance of a number of engineering studies and nondestructive tests that evaluated system components for degradation caused by thermal cycling, erosion, corrosion, and routine wear. The objective of the assessment was to identify both existing and future equipment degradation problems. The equipment assessment, combined with the established maintenance history of the facility, provides a solid basis for evaluating the existing incinerator components for maintainability and future availability.

\subsubsection{Incinerator (F)rimary and Secondary Chambers)}

A detailed fatigue analysis of both the lower and upper incinerator chambers was performed in 1992 to evaluate the effects of thermal cycling on the chambers. One thermal cycle is defined as heatup, steady state, and cooldown operations of the incinerator. The fatigue analysis results indicate that, at a thermal cycling rate of 20 times per year, the WERF incinerator could theoretically operate for more than

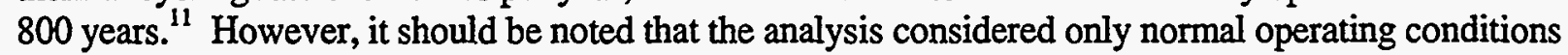
and did not consider localized high heating effects.

In 1992, a crack in the PCC shell was discovered directly across from the PCC burner.

Consequently, the interiors of both the primary and secondary chambers were inspected by a field engineer supplied by the incinerator manufacturer (Environmental Control Products). Additionally, an ultrasonic test was performed on the PCC to verify that erosion/corrosion behind the refractory had not reduced the wall thickness of the incinerator shell to below design specifications. ${ }^{12}$ 
The upper chamber and supporting equipment were found to be in relatively good condition. The refractory in the chamber had sustained considerable wear, but was not in need of replacement. Results of the ultrasonic test analysis on the lower chamber indicated that little, if any, corrosion/erosion of the shell had occurred. The lower chamber internals were found in poor condition. The chamber refractory had deteriorated to the extent that it required complete replacement. All of the underfire air tubes were also in need of replacement. Repairs were made to the chamber as recommended by the field engineer. ${ }^{12}$

Currently, the PCC refractory is in excellent condition. It was replaced in 1992 and has sustained nearly 10,000 hours of operations since installation, with little detected wear. It is likely that the refractory will withstand a minimum of 10,000 additional hours of operations and possibly, 20,000 additional hours. This is a significant improvement when compared to the original refractory, which required replacement after only 10,000 hours of operation. The improvement can be attributed to better materials of construction and a more controlled heatup/cooldown process. In addition, WERF personnel inspect the inside of the chambers after the conclusion of each incineration campaign. If damaged, the refractory is repaired prior to the next incineration campaign, preventing accelerated refractory wear in the damaged area.

The SCC refractory is currently in need of replacement. It has never been replaced and has sustained nearly 20,000 hours of incinerator operations. With the installation of an improved refractory material (e.g., rammable, cured brick), it is reasonable to assume that the life expectancy of the new refractory will double.

The thermal heating and cyclic effects on the reinforced concrete floor supporting the incinerator were evaluated in 1992 when cracks in the concrete floor were detected. Off-normal events, such as seismic activity, were not considered in the evaluation. It was found that temperatures approaching $190^{\circ} \mathrm{F}$ at the surface of the concrete caused by incinerator operations do not pose a significant risk to the concrete floor. ${ }^{13}$ However, it is difficult to design reinforced concrete to withstand elevated temperatures due to the effect temperature has on concrete quality, the nonlinear behavior of concrete at elevated temperatures, and the lack of adequate code provisions for concrete design at elevated service temperatures. Cracking of the concrete can result; therefore, it was recommended that the concrete floor beneath the incinerator be inspected periodically for additional cracks. To date, no additional cracking has been discovered.

\subsubsection{Bottom Ash Handling System}

The residue that remains in the incinerator PCC from burning of waste is referred to as bottom ash. The bottom ash handling system removes ash from the PCC and into containers for subsequent stabilization by solidification. The system is located in the PER-609 basement and is sealed and situated directly under the PCC. Bottom ash in the PCC is pushed into the ash hopper by the action of the ash ram. The hopper is equipped with a cooling air system to cool the ash while it is in the hopper. Clinkers or fused ash (slag) are routinely formed as a result of operating the incinerator. As a result, the bottom ash handling system was designed to handle clinkers. The clinkers are pushed into the bottom hopper by the ash ram.

Currently, the bottom ash handling system could be a potential limiting factor in the duration of an incinerator campaign. The bottom ash handling system accepts bottom ash from the incinerator at temperatures of $150^{\circ} \mathrm{F}$ or less. Incinerator operations are then suspended to allow the ash to cool prior to its transfer to the ash handling system. Because bottom ash currently is not transferred from the incinerator to the ash handling system during incinerator operations, an incineration campaign could be limited by the bottom ash capacity of the PCC. This, of course, is dependent on the ash content of the waste being processed and the scheduled length of the campaign. 


\subsubsection{Heat Exchanger}

The heat exchanger is a single-pass, shell-and-tube design. Hot incinerator gas passes through the tube side of the heat exchanger and is cooled by outside air that is passed through the shell side. The shell side cooling air is discharged through the south stack. The tube portion of the heat exchanger is constructed of stainless steel. The shell side is constructed of carbon steel.

The heat exchanger has been a maintenance problem since first installation and on occasion, has been a limiting factor in the duration of an incineration campaign. While incinerating, particulate carried over from the incinerator is deposited in the heat exchanger tubes. The buildup decreases the crosssectional area of the tubes causing a flow restriction for the offgas exiting the incinerator. This results in a higher than designed pressure drop across the heat exchanger. If the pressure drop across the heat exchanger gets too high, an adequate vacuum in the incinerator cannot be maintained and incinerator operations must be suspended. After the heat exchanger has cooled, WERF personnel can enter the offgas duct and clean the interior of the heat exchanger tubes. The cleaning method reduces the wall thickness of the tubes over time and weakens the heat exchanger tube sheet welds. Eventually, the tubes fail and must be capped. As tubes are capped, the effective heat transfer area of the heat exchanger is further reduced. The pressure drop across the heat exchanger (when clean) increases with the capping of each tube until the pressure drop becomes unacceptable. The heat exchanger is then replaced.

A number of corrective actions have been taken recently to minimize heat exchanger tube fouling. These include new tube cleaning techniques and the reduction of air in-leakage into the incinerator, which promoted particulate carryover to the heat exchanger. The corrective actions have worked to a limited degree.

WERF installed its third heat exchanger in October 1996. The average life expectancy for the heat exchanger is 8,000 hours based on past maintenance history. If the WERF continues to operate for the next 10 years, it is likely that the heat exchanger would require four-six replacements at a total equipment and installation cost of over $\$ 1,000,000$.

The cooling process provides the ideal temperature and associated gas residence times for $\mathrm{D} / \mathrm{F}$ formation. Municipal waste incinerators equipped with heat recovery units similar in design to the WERF heat exchanger experience higher D/F emissions than those which have a rapid offgas cooling mechanism. ${ }^{1}$

\subsubsection{Baghouse Dust Collectors}

The baghouse dust collector consists of two baghouse units installed in parallel. Each collector is divided into two dust-tight compartments. The lower compartment is called the housing and contains the filter bags and cages. The upper compartment is called the plenum and houses the blowtubes, supports the solenoid valves, diaphragm valves, compressed air manifold, and an exhaust outlet for the filtered gases. Each baghouse contains 144 filter bags approximately 100-in. long in 12 rows of 12 bags. The bags are supported from the tube sheet and held rigidly open by carbon steel wire cages inserted into each bag. Integral with each cage is a venturi nozzle that sits on the tube sheet and directs the compressed air jet into the center of the bag. The filter bags are fabricated from a highly texturized fiberglass fabric. A pyramidal shaped hopper is connected to the bottom of each collector. The hopper provides an inlet for the dust-laden gases and an outlet for the collected dust. At the inlet, a diffuser absorbs the impact of the high velocity dust particles and disperses the flow in an even distribution to the filter bags. At the hopper outlet is a rotary airlock that provides a valve between the filtration system and the dust transfer system. 
Ultrasonic testing of the baghouse was performed in 1992 to verify that erosion/corrosion had not reduced the housing wall thickness to below design specifications. Results of the test showed that no significant material degradation has occurred. ${ }^{14}$

The baghouse has no serious maintenance issues. Baghouse bags and cages are considered consumables and are replaced routinely when the bags become loaded with particulate. Differential pressure across the baghouse dust collectors is measured continuously and is used as an indicator for bag replacement.

\subsubsection{Dust Transfer System}

The flyash handling system consists of the pneumatic transfer equipment, a baghouse collector, gate valve, and a table lift. Flyash contained in the hoppers of the main baghouse dust collectors is transferred by rotary valves to the pneumatic transfer line. An electric heater heats the transfer air to reduce the relative humidity of the air and prevent the ash from absorbing moisture and agglomerating. Heated air and flyash are pulled through the transfer line by a fan in the ash handling room. The transfer air is pulled into the transfer baghouse in the ash handling room. After the flyash is collected in the transfer baghouse, it drops into a steel container staged below the baghouse. A transition piece at the bottom of the transfer baghouse provides a pressure seal and bagout fitting to seal the container from the room and personnel during transfer operations.

Currently, the flyash collected in the baghouse dust collectors is removed in one of two ways: (1) the flyash can be pneumatically transferred via the dust transfer system as described above, or (2) the flyash can be manually removed from the baghouse hoppers. In either case, the flyash is placed in a 55-gallon or 71-gallon steel container and stored in the temporary accumulation area while analysis of the flyash is accomplished. The preferred method of flyash removal from the system is using the dust transfer system for two reasons. First, personnel exposure to radioactive and hazardous constituents is minimized. Second, flyash can be removed from the system continuously while incinerating, which eliminates the baghouse hopper capacity from becoming a limiting condition in the duration of an incineration campaign.

Unfortunately, the dust transfer system has proven itself unreliable due to miscellaneous mechanical failures of equipment. On numerous occasions, WERF personnel have had to manually remove flyash from the main baghouse dust collectors after the completion of an incineration campaign. Fortunately, no incineration campaign to date has been cut short due to the inoperability of the dust transfer system. However, manned entry into the baghouse is becoming a growing concern due to the high concentration of heavy metals present in the flyash that become airborne during ash removal.

\subsubsection{Main Off gas HEPA Filter Housing}

The main HEPA filtration unit is installed downstream of the baghouse. The unit consists of a stainless-steel housing ( 4 filters wide $\times 4$ filters high), prefilter racks each holding 16 prefilters, HEPA filter racks holding a single-stage array of $16 \mathrm{HEPA}$ filters and bagout features. The prefilters are installed to protect the HEPA filters and are the industrial-grade, separator-type filters with stainless steel frames. The HEPA filters are nuclear grade, separatorless-type, and constructed using acid-resistant media with stainless steel frames.

Rust colored hairline cracks were discovered on the bottom of the HEPA filter housing in 1992. Using a dye penetrate test, it was determined that the cracks penetrated through the base material of the housing. An ultrasonic test was then performed to determine the filter housing base metal thickness and indicated that there was no reduction in the base metal thickness. After discussions with the 
manufacturer, it was determined that the cracks correlated directly with a stitch weld that secures the housing stiffener to the housing. It was concluded that the cracks were caused from residual thermal stresses created during manufacturing. The external bottom surface of the housing was coated with rustinhibiting high temperature paint. The housing internals are inspected periodically during filter change out. No additional cracking has been detected. ${ }^{15}$

There are no maintenance problems associated with the HEPA filtration unit. Prefilters and HEPA filters are considered consumables and are replaced periodically when the filters become loaded with particulate. Differential pressure across the filtration unit is measured continuously and is used as an indicator for filter replacement.

\subsubsection{Air Pollution Control System Duct-Work, Fans, and Stack}

The APCS duct-work is constructed primarily of stainless steel, and it provides a flow path for the offgas exiting the incinerator to downstream APCS components. The duct-work is heavily insulated to prevent both heat loss out of the ducting and the formation of condensate inside the ducting during operation of the incineration process. The APCS uses two induced draft fans configured in parallel as the primary method of maintaining the incinerator and APCS at a negative pressure. The fans have a combined capacity of 16,000 acfm at $350^{\circ} \mathrm{F}$ and $20 \mathrm{in}$. water column. Incinerator offgas is discharged from the process out of the north stack, which is $49-\mathrm{ft}$ tall. The stack is constructed of 0.25 -in. carbon steel.

Ultrasonic testing of the ductwork was performed in 1992 to verify that erosion/corrosion had not reduced the wall thickness to below design specifications. Results of the test showed that no significant material degradation has occurred. ${ }^{14}$

There are no maintenance problems associated with the system main induced draft fans. The fans are inspected and lubricated routinely. Periodically, the fan belts and bearings are replaced. Other components such as the fan shaft and impeller are also inspected at this time and replaced, as necessary.

Corrosion was first identified on the north stack in localized areas near the baffle plate in 1989. The baffle plate is a 0.25 -in. thick piece of carbon steel welded at a $45^{\circ}$ angle inside the stack. At the low point of the baffle is a drain. The baffle plate and drain provide a water collection trap above the stack foundation, allowing offgas vapor condensation, rain, and snow to temporarily collect prior to draining. Corrosion in the baffle plate area of the stack was monitored periodically, and by 1991, it was determined that the condition of the baffle area of the stack was deteriorating. Ultrasonic testing of the stack was performed to determine the remaining wall thickness. The areas selected for testing were based on the pattern of the previously identified corrosion. The test results indicated that extensive stack corrosion was taking place that was not visible from outside the stack. A sample of the badly corroded area above the baffle plate was then removed for detailed corrosion analysis. The cause of the corrosion was determined to be concentration of chlorides in accumulated water on the baffle plate. It was also determined that the problem could have been mitigated by continuously draining the stack. The corroded area of the stack was weld-repaired, and the drain system reconfigured so that it would drain continuously. An ultrasonic test is now performed anrually on the stack. Since the stack was repaired and modified, no further corrosion has been detected. ${ }^{16}$

\subsubsection{Oxygen, Carbon Monoxide, and Hydrogenchloride Analyzers}

The $\mathrm{O}_{2}, \mathrm{CO}$, and $\mathrm{HCl}$ analyzers are relatively reliable and accurate instruments. However, the sample gas-conditioning unit associated with the $\mathrm{HCl}$ analyzer requires a high degree of maintenance and engineering support. The poor reliability of the sample gas-conditioning unit periodically hinders 
incinerator waste processing operations, which has a direct impact on production schedules. The sample gas-conditioning unit is currently the weak link in the capability to continuously monitor $\mathrm{HCl}$ at WERF.

\subsection{Conclusions and Recommendations}

The assessment of the existing WERF incinerator and associated APCS identified no equipment-related issues that would act as a deterrent to upgrading the WERF to comply with MACT standards. The WERF incinerator and APCS have an established maintenance history resulting from years of operation. The existing incinerator and APCS are well maintained and can likely be expected to operate another 10 years. Consumable items such as refractory, baghouse bags, and HEPA filters will require replacement periodically as part of the maintenance program. The proposed WERF upgrade to meet the MACT rule will eliminate the problems associated with the offgas heat exchanger and the flyash transfer system. This will increase the incinerator throughput and availability.

The following equipment repairs and modifications are recommended and should be considered regardless of proposed facility upgrades:

- Replace the refractory in the SCC. The chamber refractory is in need of replacement, regardless of proposed facility upgrades to comply with MACT requirements. It may be possible to coordinate a plant outage to replace the refractory with a temporary shutdown of the WERF incineration process to comply with MACT standards.

- Replace the incinerator lower and upper chamber burner systems. New burners have been procured and are readily available for installation. Advantages of replacing the existing burners include better combustion efficiency, less fuel oil carryover to downstream APCS components, longer offgas residence times, and reduced offgas volume flowrates.

- Attempt to transfer during incinerator operations using the current system configuration, as required, so that ash can be removed from the incinerator while the incinerator is operating. The primary chamber would no longer be required to contain the bottom ash generated from an entire incineration campaign thus eliminating a potential limiting condition of operation.

- Eliminate the heat exchanger from the APCS and cool the process offgas using a different method. Although heat exchanger replacement can be anticipated, this maintenance activity is expensive and time consuming. It can be a limiting factor in the duration of an incineration campaign. An additional problem is related to $\mathrm{D} / \mathrm{F}$ formation.

- Repair or replace the existing baghouse flyash handling system if a larger waste throughput or campaign is desired. The existing system is unreliable and has limited capacity.

- $\quad$ Provide redundant CEMs. 


\section{SCREENING COMMERCIALLY AVAILABLE AIR POLLUTION CONTROL TECHNOLOGIES}

The study team reviewed the various APC technologies for their ability to meet the MACT proposed rule regarding $\mathrm{HCl}, \mathrm{Hg}$, and $\mathrm{D} / \mathrm{F}$ control, and the technology descriptions are listed below according to their ability to control $\mathrm{HCl}, \mathrm{Hg}$, and $\mathrm{D} / \mathrm{F}$ emissions. Some technologies are described in more than one section because of their ability to treat more than one contaminant.

\subsection{Hydrochloride Control Technologies}

Many types of devices are used in industry to remove acid gases. All rely upon the same principle: the conversion of acid to a salt by reacting with an alkali reagent. Once converted, the salt can be collected as a dry particulate or dissolved in a liquid solution. Several alkali reagents are used for acidgas removal; however, the most commonly used reagents are either sodium- or calcium-based. The choice of which reagent to use depends upon whether the process is wet, dry, or semidry, and other considerations, such as solubility and secondary waste disposal. The following technologies were identified and evaluated as acid-gas treatment options.

\subsubsection{Wet Scrubber Technology}

Wet scrubber technology is based on capturing particulate matter and absorbing acid gases from the gas phase into the liquid phase. Therefore, the wet scrubber design is optimized for liquid droplet creation and providing intimate contact between the droplet and the gas stream. The type of wet scrubbing technologies considered, include the following.

4.1.1.1 Packed Bed Scrubbers. Packed bed scrubbers are vertical vessels filled with random packing, structural packing, or mesh pads. The gas stream enters the bottom of the packed bed through a flow distributor and flows upstream from the aqueous stream, which cascades down through the packing material or mesh. The offgas should be saturated prior to entry into the packed bed scrubber.

4.1.1.2 Tray Column Scrubbers. Tray column scrubbers are vertical columns with one or more trays installed transversely to the counter-current gas and liquid flows. The trays are the components used to accomplish liquid/gas contact. The liquid phase enters near the top of the tower and flows across the first tray, then cascades down to the next tray, etc. Each tray has an overflow wire that is used to control the depth of the liquid on the tray. Meanwhile the gas phase is introduced to the bottom of the column and flows upward through tray openings, which are designed to promote gas and liquid contact.

4.1.1.3 Fluidized-Eled Scrubbers. Fluidized-bed scrubbers look like packed bed scrubbers but are operated with high gas and scrub solution flowrates. The bed packing, usually spherical in shape, is flooded with liquid and fluidized by the turbulent motion of the passing gas and liquid streams.

4.1.1.4 Spray Scrubbers. Spray scrubbers are relatively simple processes that use vertical towers with countercurrent gas-liquid flows. The gas inlet is located in the side of the tower near the bottom. The offgas typically passes through a gas distribution plate to evenly spread the gas flow up through the tower, and the liquid is sprayed through nozzles located at the top of the column downward into the gas stream. The nozzles can be replaced by mechanical rotary atomizers, which produce small sized liquid droplets necessary for efficient heat and mass transfer between the phases.

4.1.1.5 Venturi Scrubbers. Venturi scrubbers, a form of high-energy scrubbers, mix the scrubbing liquid and offgas together as they accelerate through a converging section of ductwork, which 
transforms into a narrow throat. The liquid and gas then flow together out through a diverging section. The high gas velocity in the throat section shears the scrubbing liquid into a cloud of very fine droplets that capture particulate matter. A disadvantage for using a venturi scrubber includes a relatively large, gas-side pressure drop, which is necessary for fine droplet formation and particulate collection. These pressure drops may exceed 30-in. of water column and are expensive to operate due to larger power consumption.

4.1.1.6 Free-Jet Scrubbers. Free-jet scrubbers include steam or air-driven ejectors followed by free-jet nozzles. The ejector nozzle is located inside the offgas duct, and the offgas flows around the outside of the nozzle while steam, air, or some other compressible fluid is discharged from the ejector nozzle at speeds of $3200 \mathrm{ft} / \mathrm{sec}$. Near the ejector nozzle exit, water is injected into the high velocity flow. The collision of the water with the high velocity offgas/compressible fluid mixture produces shock waves, which in turn create very small droplets. The offgas, water droplets, and steam mixture then pass through a free-jet nozzle that is mounted inside an expanded chamber. The fine droplets agglomerate to form larger droplets that contain the particulate. These are readily removed via a moisture separator.

4.1.1.7 Ionizing Wet Scrubbers. Ionizing wet scrubbers can effectively remove acid gases, particulate, and condensable organics. The collection of particulate involves a combination of technologies using electrostatic particle charging, inertial impaction, and a phenomenon referred to as image force attraction. Flue gases are first cooled and saturated in a quencher. The gas stream is then passed through a high voltage ionizing chamber to electrostatically charge the particulate, much like a conventional electrostatic precipitator.

4.1.1.8 Froth Scrubbers. Froth scrubbers simply cause the offgas to flow upward through a liquid bath. They are used for gas cooling and can remove both large and submicron particles. These scrubbers can also neutralize acid gases when alkali is added to the liquid bath. The froth scrubbers are designed to generate a froth zone by balancing liquid and gas momentums as the gas is bubbled through the liquid. The froth zone results in a large interfacial area, that provides the intense mixing and turbulence necessary for good mass and heat transfer.

\subsubsection{Semi-dry Scrubber Technology or Spray Dry Absorber}

The spray dry absorber (SDA) is the only technology that is categorized as a semidry scrubber. This technology is referred to as semidry because a wet caustic reagent is used as the scrub solution but the residue produced is dry. The dry residue has the advantage of being easier to dispose in comparison to a wet residue, which is a major reason for the development and use of this technology. The following section describes this technology in more detail.

An SDA is designed to partially quench the offgas while removing the acid gases by neutralizing them with an alkali reagent. Unlike the wet scrubber technology, the stream exiting the SDA process does not contain liquid. Water and alkali reagents are mixed together and injected into the top of the absorber as a finely atomized spray using either a two-fluid nozzle or a rotary atomizer. As the liquid droplets and gas flow downward together through the vessel, heat from the offgas evaporates the water, which in turn cools the gas. Simultaneously, the acid gases present in the flue gas absorb into the small liquid droplets and react with the alkali reagent to form a salt. The vessels required for this simultaneous offgas cooling and acid gas neutralization are relatively large since gas residence times on the order of 4 20 seconds are required. 


\subsubsection{Dry Scrubber Technology}

Dry acid-gas removal technology has been developed in an effort to lower the cost of operations. Less costly alkali reagents, such as hydrated lime, can be used without the need for alkali preparations. Because a dry secondary waste is generated, there is no need for wastewater treatment facilities or drying equipment. The following sections discuss two dry acid-gas removal systems.

4.1.3.1 Dry Sorbent Injection. Dry sorbent injection (DSI) systems introduce a dry alkali reagent directly into the offgas stream. For DSI, the offgas from the incinerator is typically cooled from around $2,000^{\circ} \mathrm{F}$ to less than $450^{\circ} \mathrm{F}$ to make it suitable for handling in the downstream equipment. A dry powder sorbent such as hydrated lime or sodium bicarbonate, is either gravity fed or pneumatically injected into the offgas duct at a point where the sorbent reacts with the acid gases and converts them to solid particulate. Although a part of the acid-gas neutralization takes place in the ductwork, most of the reaction occurs in a baghouse located downstream of the point where the dry sorbent was fed. The unused reagent builds up in the filtercake on the baghouse filters. This mechanism provides the intimate contact between the solid and gas phases, which is necessary for the acid gas reaction to occur. The reaction products are then removed with the fly ash when the bagfilters are cleaned.

4.1.3.2 Dry Fluidized-Bed Scrubbing. Dry fluidized-bed scrubbing consists of vertical vessels filled with solid alkali reagent such as hydrated lime or sodium bicarbonate. The offgas flows upward through the reagent bed with sufficient velocity to fluidize the particles. The random action of the fluidized particles provides the solid and gas phase contact necessary for acid-gas neutralization. Unfortunately, this feature also promotes inefficient reagent usage since it is impossible to remove the solid reactant products without removing significant unreacted reagent.

\subsection{Mercury Control Technologies}

An understanding of the chemistry and thermodynamics of $\mathrm{Hg}$ and its compounds is necessary to identify the best technology to effectively remove $\mathrm{Hg}$ from an incinerator offgas. Although the $\mathrm{Hg}$ thermodynamic and chemical properties, as well as its behavior in thermal treatment systems, have been reviewed, ${ }^{17}$ actual incineration and offgas treatment processes may not actually achieve chemical equilibrium conditions. However, equilibrium calculations can still provide qualitative information regarding mercury speciation. ${ }^{18,19}$

At gas temperatures above $1,020-1,300^{\circ} \mathrm{F}$, gaseous elemental $\mathrm{Hg}$ is thermodynamically and kinetically favored over all oxidized species, even in the presence of high $\mathrm{HCl}$ levels. ${ }^{18,20,21}$ At temperatures below this range, mercury dichloride $\left(\mathrm{HgCl}_{2}\right)$ is thermodynamically, but not kinetically, favored. ${ }^{9,22}$ While gaseous elemental $\mathrm{Hg}$ may not be thermodynamically favored at the lower temperatures, a rapid temperature quench, which is common in many incinerator systems, may prevent elemental $\mathrm{Hg}$ from being converted to $\mathrm{HgCl}_{2}$ on a short-term basis. ${ }^{23}$

Equilibrium models have shown that other $\mathrm{Hg}$ species, such as $\mathrm{HgO}$, are not thermodynamically favored at either high or low offgas temperatures, and the degree of mercury oxidation is influenced by the presence of other pollutants. The formation of oxidized $\mathrm{Hg}$ species increases with increasing concentration of sulfur dioxide, $\mathrm{HCl}, \mathrm{Cl}_{2}$, and nitrogen oxides. ${ }^{9}{ }^{24}$ The gas-phase reaction of mercury with chlorine gas is relatively quick even at temperatures as low as $50^{\circ} \mathrm{F}$.

Mercury and mercury compounds exhibit relatively high vola tility for metal compounds. ${ }^{17}$ The vapor pressures of both $\mathrm{Hg}$ and $\mathrm{HgCl}_{2}$ are high enough that condensation at low temperatures is insufficient to reduce their vapor phase compositions below the proposed emission standards. Mercury "dichloride is highly water soluble while elemental mercury is very insoluble in typical scrub solutions. ${ }^{25}$ 
Other pertinent information concerning $\mathrm{Hg}$ chemistry include the following:

- Oxidation of elemental $\mathrm{Hg}$ vapor is catalyzed by activated carbon. ${ }^{26}$

- Mercury amalgams may occur with some metals such as gold, silver, and platinum. ${ }^{27}$

A list of current and proposed technologies that exploit the physical and chemical properties of $\mathrm{Hg}$ in order to remove it from an incinerator offgas are reviewed below.

\subsubsection{Particulate Matter Control Device}

The purpose of PM control devices, such as a fabric filter baghouse, is to capture the entrained particulate and condensed volatile metals that result during the cooling of the offgas. As a result, the PM devices will probably remove some $\mathrm{Hg}$ from the incineration offgas when it captures entrained PM from the incinerator offgas. Mercury and its compounds can be removed from an incinerator offgas by absorption onto fly ash material, that contains magnesium or carbon. Particulates with high surface areas can be generated from the incomplete combustion of organic material. ${ }^{28}$ Since it is desirable to operate incinerators under conditions that minimize ash entrainment, and the amount of absorbent in the ash is dependent on the composition of the waste and incinerator operating conditions, it is not prudent to assume significant $\mathrm{Hg}$ removal can occur by capturing PM.

It has been observed that calcium chloride $\left(\mathrm{CaCl}_{2}\right)$-impregnated carbon possesses significant $\mathrm{Hg}$ adsorptive capacity. ${ }^{29}$ Calcium-based solids added upstream of a $\mathrm{PM}$ control device to remove $\mathrm{HCl}$ in the offgas will form a thin cake on the baghouse filters. As the $\mathrm{HCl}$ is removed, $\mathrm{CaCl}_{2}$ is formed. It is unclear if any significant $\mathrm{Hg}$ removal would be achieved in a baghouse containing $\mathrm{CaCl}_{2}$ without the presence of activated carbon.

\subsubsection{Wet Scrubbing}

Wet scrubbing is commonly used in thermal process offgas systems for controlling particulates and acid gases. Mercury removal efficiencies for traditional wet scrubbing can vary from less than $10 \%$ to over $99 \% .^{30-38}$ Such a wide variation in mercury removal performance is the result of many different factors that affect mercury removal thermodynamics, mass transfer, or kinetics. These factors can include $\mathrm{Hg}$ speciation and species concentration in the scrubber inlet gas, scrubber design, and scrubber operating conditions.

Mercury dichloride, which is thermodynamically favored at wet scrubber operating temperatures, is also highly water soluble, while elemental mercury is very insoluble in typical scrub solutions with pHs in the range of 6-9. Some additives, such as sodium sulfide or sodium hypochlorite, may improve the removal of elemental $\mathrm{Hg}$ by oxidizing it to a water-soluble species. Activated carbon in the scrubber solution may absorb $\mathrm{Hg}$ directly from the offgas. In these cases, the wet scrubber $\mathrm{Hg}$ removal efficiency is a function of the contact efficiency between the offgas and scrubber solution. Scrubber discharge to scrubber recycle ratios are typically very low to minimize the amounts of secondary waste production. To prevent the accumulation of $\mathrm{Hg}$, the $\mathrm{Hg}$ species must be removed from the scrubber solution by a separations process (i.e., filtration) or a purge stream.

\subsubsection{Carbon Adsorption}

Contacting $\mathrm{Hg}$ contaminated offgas with activated carbon or a chemically impregnated activated carbon to remove the $\mathrm{Hg}$ compounds offers the advantage of not having to handle and treat a liquid waste stream. Carbon adsorption of mercury can be accomplished by (a) injecting dry carbon into the offgas 
upstream of a particulate matter control device (typically a baghouse), or (b) using a fixed bed of granular carbon through which the offgas flows. Activated carbon readily captures oxidized forms of mercury, such as $\mathrm{HgCl}_{2}$ and $\mathrm{HgO}$, while removing elemental mercury more slowly by first converting it to the oxidized state. ${ }^{39}$

4.2.3.1 Activated Carbon. The effectiveness of carbon adsorption depends on many different factors including mercury concentration and its speciation, chemical pre-treatment of carbon, operating temperature, adsorbent particle size, pore size and active site distributions, gas contact time with the activated carbon, and the total $\mathrm{Hg}$ capacity of the carbon. The amount of mercury loading on activated carbon is on the order of $1 \%$ by weight; therefore, this technology is limited to offgas streams with low Hg concentrations.

4.2.3.2 Sulfur Impregnated Activated Carbon. Although activated carbon only has been used to remove low concentrations of mercury from large incineration offgas streams, it has a very limited mercury retention capacity and the mercury adsorption is readily reversible (i.e., the mercury is easily desorbed from activated carbon). ${ }^{29}$ Activated carbon impregnated with sulfur or chemicals that can react with $\mathrm{Hg}$ significantly increases the $\mathrm{Hg}$ loading capacity of the carbon. This form of adsorption is referred to as chemsorption where the mercury reacts with the chemicals impregnated in the carbon to form mercury compounds that have very low volatility and can be retained in solid form on the carbon surface.

Sulfur-impregnated activated carbon significantly increases the mercury retention capacity because the sulfur reacts with the mercury to form mercury sulfide, which is a more thermally stable compound than elemental or mercury oxide. Sulfur-impregnated carbon has loading capacities up to $20 \mathrm{wt} \%$. The method used for impregnating granular activated carbon with sulfur has a pronounced effect on mercury removal capacity. ${ }^{40}$ Chemical bonding of sulfur at $1,115^{\circ} \mathrm{F}$ provides a more uniform distribution of sulfur throughout the granular activated carbon pore structure than is achieved by conventional condensation techniques, yielding improved performance. ${ }^{29}$

The extent of $\mathrm{Hg}$ removal from the offgas with sulfur-impregnated carbon is also a function of gas contact time with the carbon, Hg concentration in the offgas, carbon particle size, and gas turbulence. The theoretical equilibrium adsorption capacity of one type of sulfur-impregnated carbon is $85 \mathrm{~g}$ of mercury per $100 \mathrm{~g}$ of adsorbent. However, it is impractical to achieve that level in commercial applications because an extremely long contact time would be required to obtain diffusion of the mercury into the pellets and for the chemical conversion to take place. Dynamic adsorption capacity data obtained by carbon vendors suggests that a typical design capacity of carbon for mercury is $20 \mathrm{wt} \%$ of the carbon. 40

One study determined that the carbon capacity for $\mathrm{Hg}$ increased with the concentration of the $\mathrm{Hg}$ in the gas, indicating that there was better utilization of the carbon with the greater adsorption driving force provided by the higher concentration. ${ }^{41}$ The adsorptive capacity of the carbon for mercury at low temperatures and higher inlet concentrations of mercury was nearly double its adsorption capacity at the same temperature but about half the inlet mercury concentration. At low concentrations of mercury in the offgas, the adsorption process could become mass transfer limited and reduce mercury removal efficiency. When the system is mass transfer limited, using smaller carbon particles can improve mercury removal.

Although the extent of $\mathrm{Hg}$ removal with activated carbon with no chemical impregnation generally decreases with increasing temperature, the rate of $\mathrm{Hg}$ removal by chemi-sorption on sulfur-impregnated carbon increases with temperature to a maximum value beyond which sulfur or mercury sulfide begin to volatilize. ${ }^{29}$ The maximum operating temperature for sulfur-impregnated carbon depends upon the how it is made. The product marketed by Calgon Carbon has a maximum operating temperature of $95^{\circ} \mathrm{F}^{41}$ 
Nucon International offers a sulfur-impregnated activated carbon, which it claims will perform without volatilization of material from its carbon at temperatures as high as $390^{\circ} \mathrm{F} .{ }^{40}$ Nucon International also noted a case study in which good $\mathrm{Hg}$ removal had been achieved using a sulfur-impregnated carbon bed with operating temperatures as high as $250^{\circ} \mathrm{F}$.

4.2.3.3 Carbon Injection. Carbon injection is becoming more widely used in European waste incineration plants for controlling $\mathrm{Hg}$ emissions. The method of injection and gas flow characteristics must be carefully controlled to allow proper mixing, temperature, and contact time. Baghouse collection of the spent injected carbon affords increased contact time with mercury in the flue gas (thus enhancing removal) because the injected carbon particles that are captured on the bags can continue to absorb mercury from the gas that flows through the bag filters. Carbon injection systems can be added relatively easily to offgas systems that are already equipped with baghouses for PM removal and the carbon injection adds little to overall pressure drop of a baghouse. These features make carbon injection more attractive compared to fixed carbon beds to industries such as industrial and utility power generation where baghouse PM control is common, offgas flowrates are large, and $\mathrm{Hg}$ concentrations are low. Carbon injection systems typically operate at temperatures between $150-200^{\circ} \mathrm{C}\left(300-400^{\circ} \mathrm{F}\right)$. Few systems operate at temperatures less than $150^{\circ} \mathrm{C}$ to avoid moisture condensation in downstream PM removal equipment, and the mercury adsorption capability of the carbon is significantly decreased above $200^{\circ} \mathrm{C}$.

4.2.3.4 Fixed Bed Carbon Adsorption. In a typical, fixed-bed carbon adsorption system, the flue gas flows through a vessel packed with a specified depth of the carbon granules. The bed and packing are designed to limit the linear velocity of the offgas in the bed to increase the contact time with the carbon. Due to the increased contact times and typically lower operating temperatures, better removal efficiencies can be achieved than for carbon injection. ${ }^{40}$

Fixed-bed carbon adsorption is most applicable for processes that generate small offgas flowrates with high offgas $\mathrm{Hg}$ concentrations. Since the WERF incinerator system has a relatively low offgas flowrate and potentially high mercury concentrations, a fixed-bed carbon adsorber is an applicable technology for $\mathrm{Hg}$ control. Carbon beds can be designed so that they are replaced or sized to last for the lifetime of the facility.

\subsubsection{Gold Amalgamation Filter.}

Mercury can be removed from offgas streams by the reversible sorption of Hg on noble metal sorbents such as gold, selenium, or gallium dispersed on a fixed-bed of support media such as alumina. The most common metal used is gold because of its affinity for $\mathrm{Hg}$ regardless of the $\mathrm{Hg}$ speciation. Mercury removal efficiencies as high as $99.99 \%$ and effluent $\mathrm{Hg}$ concentrations in the treated offgas as low as 3 to $8 \mu \mathrm{g} / \mathrm{m}^{3}$ have been measured..$^{27,42,43}$ Operating temperatures up to $180^{\circ} \mathrm{C}\left(350^{\circ} \mathrm{F}\right)$ are possible, and there can be relatively low offgas pressure drops. Tests indicate that mercury removal is independent of mercury speciation, water, and $\mathrm{HCl}$ in the flue gas. The gold can be regenerated by taking the filter bed off-line and thermally desorbing the mercury into a small flowrate of purge gas. The purged mercury, at very high concentrations in the purge gas, can be largely recovered as liquid mercury upon cooling. The remaining mercury in the purge gas can be vented through a second gold amalgamation filter that is on-line while the first filter is being regenerated. 


\subsection{Dioxin/Furan Control Strategies}

Polychlorinated dibenzodioxins and polychlorinated dibenzofurans (D/Fs) congeners are subject to strict emission limits. In order to meet these limits, the formation of $\mathrm{D} / \mathrm{Fs}$ must be inhibited or a significant quantity of these compounds must be captured before the offgas is released to the environment. Dioxins and furans are generally formed downstream of combustion units. The mechanism for their formation is not well understood, but one example is called the de novo synthesis reaction. Key features of this synthesis reaction include the following: ${ }^{44}$

- The effective reaction temperature range is $250-500^{\circ} \mathrm{C}\left(480-930^{\circ} \mathrm{F}\right)$. Within this range, the kinetics of the synthesis reactions are significant, and the compounds formed will remain stable as long as the offgas temperature is not reheated to combustion temperatures $\left(\sim 1,700-2,000^{\circ} \mathrm{F}\right)$.

- Precursors must be available for $\mathrm{D} / \mathrm{F}$ formation. These include elemental chlorine and organic compounds resulting from incomplete combustion (preferably ring compounds). Chlorine is formed by the deacon reaction of $\mathrm{HCl}$ and oxygen. The organic compounds are then partially oxidized and chlorinated to form D/Fs. Although only a small fraction of $\mathrm{HCl}$ is converted to elemental chlorine, the amount of chlorine generated can be orders of magnitude greater than necessary to generate detectable quantities of $\mathrm{D} / \mathrm{F}$.

- The presence of sulfur dioxide suppresses the formation of D/Fs. This has been observed in coal combustion processes where conditions are ideal for $\mathrm{D} / \mathrm{F}$ formation.

- $\quad$ Catalytic surfaces appear to be necessary to maximize D/F formation. The more highly chlorinated congeners are typically found on PM and solid surfaces in the offgas scrubbing system rather than the offgas itself. Particulate matter in general has an effect with certain compounds being more active than others.

\subsubsection{Minimize Formation}

Given the observations described above, it is possible to develop strategies to minimize D/F formation. One strategy is to minimize the presence of organic compounds by ensuring that good combustion practices are employed, such as proper control of waste and air flowrates, high temperatures in the incinerator combustion zone, minimal ash carryover, good gas mixing, and sufficient oxygen concentrations. After the incinerator, a quick quench process that immediately cools the offgas to adiabatic saturation significantly reduces the time the gas is in the optimum formation temperature range. Typically, these include wet offgas scrubbing or evaporator coolers that use water to quickly cool the offgas to below $400^{\circ} \mathrm{F}$. This fast cooling minimizes the gas residence time required for the $\mathrm{D} / \mathrm{F}$ production to occur. This strategy is commonly used by at least three facilities with low dioxin emissions are referenced here. ${ }^{45}$

\subsubsection{Removal}

A second strategy is to remove the D/Fs present in the offgas. Efficient particulate removal removes catalytic flyash, as well as large particles containing highly chlorinated D/F congeners. Short of re-incinerating the offgas to destroy the small quantity of D/Fs, removal from the offgas is generally accomplished by adsorption onto activated carbon. This, in turn, can be achieved by injecting carbon into the offgas stream upstream of a particulate collection device or by passing the gas over a fixed carbon bed. Several incineration processes have used a carbon injection system. ${ }^{45,46,47}$ Removal efficiency is a 
function of the contact between the gas and carbon, temperature, and contaminant concentration. Typically, gas temperatures less than $200^{\circ} \mathrm{C}\left(390^{\circ} \mathrm{F}\right)$ are required for efficient $\mathrm{D} / \mathrm{F}$ removal.

\subsection{Other Technologies}

There are numerous technologies including waste feed additives and cryogenic systems that have been used for offgas emissions control. These systems have not been included in this discussion because they are considered too experimental or expensive to be viable for the WERF MACT solution.

\subsection{Continuous Emission Monitor System Technologies}

Compliance with MACT will require that an incinerator facility have monitors to continuously measure PM, THC, and CO. A review of the state-of-the-art technology available is summarized below.

\subsubsection{Particulate Matter}

Current measurements of PM are made using EPA Method $5 .^{49}$ Method 5 involves isokinetic sampling of the stack flow and collection of particles on a filter. The filter is weighed to determine the PM mass emission rate. This is a manual method and involves time-consuming analysis that provides a direct measurement of the mass of emitted PM. A need exists for PM measurement technology that is continuous, automatic, and provides real-time analysis. The output of such a PM CEMs should be indicative of the PM mass emission rate.

Vendors offer PM CEMs employing a variety of measurement principles. Commercially available PM CEMs can be categorized as optical, extractive, and probe. Optical systems measure the behavior of light in the presence of PM. Extractive systems collect a sample of PM, which is removed from the gas stream for analysis. The probe systems insert a probe in the gas stream, which comes in contact with the PM. None of these devices have received EPA approval for stack mass emissions monitoring. The reason is that most of these devices measure secondary properties of particulate in the gas stream that are related to the mass of PM. The resultant measurement must then be related to mass by using a site-specific calibration against Method 5.

The response of light-scattering based systems is typically a function of particle size and material properties although less so than for opacity-based systems. ${ }^{50}$ Specific devices are designed to have as flat a response as possible over a large portion of the size range of interest. However, this does not eliminate the potential dependence of the device response on particle size distribution. In addition, the responses of some optical techniques depend to varying extents on particulate composition. Particulate composition also affects the accuracy of all optical approaches through its effect on particle density.

Two measurement methods that insert probes into the gas flow are available. One method measures the triboelectric effect resulting from the transfer of electric charge when particles impact on a dissimilar object. The measurement consists of inserting a metal rod into the gas flow and measuring the electric current induced by the particles that strike the rod. The method is sensitive to flow velocity, particle size distribution, particle characteristics, and changes in humidity. An accoustic energy monitor measures the shock waves created by the impact of particles with a metal probe inserted into the gas flow. The device measures the number of impacts and the energy of each impact. This measurement method is not sensitive to particles less than 10 microns, but is sensitive to changes in flow velocity and particle size distribution, and must be calibrated against EPA Method 5 results.

Two extractive measurement methods are commercially available. The beta gauge uses a heated sampling probe to obtain an isokinetic sample onto a filter. At the end of the sampling period, the filter is 
removed and placed between a carbon- 14 beta particle source and a detector. The change in beta particulate transmissivity across the filter is a function of the PM mass concentration on the filter. The instrument achieves excellent sensitivity $\left(10^{-6} \mathrm{gr} / \mathrm{dscf}\right.$ for one-hour sample) and is sensitive to all particles. Potential problems concern PM fallout within the sampling probe and the intermittent, noncontinuous nature of sample collection and analysis. An ambient air monitor based on a tapered oscillating element microbalance measures the change in the natural frequency of oscillation of a microbalance beam as particles accumulate on a filter through which the sampled gas passes. This is a direct measurement of particle mass. However, the instrument is sensitive to changes in humidity and temperature. It is questionable whether this device is suitable for use in gas stack monitoring.

The tampered oscillating element microbalance air monitor is not suitable for use for stack gas analysis, and the opacity monitoring device lacks the required sensitivity. Of the remaining approaches, none makes a direct measurement of particle mass concentration, although the beta gauge calibration depends so weakly on particle properties that the calibration is considered universal and absolute. ${ }^{51}$ The other devices require a site-specific calibration against manual gravimetric measurements. The accuracy that can be obtained in this manner is not known as it depends on the stability of the calibration. This depends in turn on a stable relationship between the measured particle properties and particle mass. The best way to determine the feasibility of applying these devices as CEMs for compliance purposes is to conduct field trials at the various types of sources to be regulated. A demonstration of these devices at facilities representative of the various source categories should determine how actual variations in PM size and composition affect the accuracy of mass concentrations inferred from the measured properties.

The cost of a beta gauge instrument is on the order of $\$ 35,000$. The light-scattering instruments range between $\$ 10,000$ to $\$ 30,000$. The measurement technologies that insert probes into the gas flow range in price from $\$ 10,000$ to $\$ 15,000$.

\subsubsection{Total Hydrocarbons}

The primary principle used in THC CEM instrumentation is the flame ionization detection method. Burning hydrocarbon-free hydrogen in hydrocarbon-free air produces a negligible number of ions. A hydrocarbon sample introduced into this flame creates a large number of ions. A polarizing high voltage is applied between two electrodes around the burner jet. The generated ionization current between the two electrodes is directly proportional to the hydrocarbon concentration in the flame and is measured by an electrometer amplifier. For processes operated at temperatures above ambient conditions, a heated CEM is required to prevent the condensation of THCs in the sample line. The cost of THC CEMs is appropriately $\$ 10,000$.

\subsubsection{Carbon Monoxide}

Standard equipment used to measure $\mathrm{CO}$ employs nondispersive infrared analyzers with gas filter correlation technology in which an infrared beam reacts with the gas. The gases absorb radiation at specific wave lengths, and this absorption is measured by a detector. This technology is currently used at the WERF to measure $\mathrm{CO}$ concentrations in the offgas. 


\section{AIR POLLUTION CONTROL SYSTEMS CONSIDERED FOR UPGRADING WERF TO MEET MACT}

The APC technologies listed above were evaluated for applicability to meet the MACT criteria for $\mathrm{HCl}, \mathrm{Hg}$, and $\mathrm{D} / \mathrm{F}$ control. After analyzing the technology and researching the chemical and physical properties for the contaminants of concern, it was concluded that no single APC technology could meet the proposed MACT limits for all three species. Rather, an APCS consisting of different APC technologies is required.

At this point in the evaluation process there were several technologies that were discontinued from further consideration:

- Most of the wet scrubbers because they require too much water or power-the packed bed, venturi, and wet electrostatic precipitator scrubbing technologies were retained as possible alternatives

- Dry fluidized bed scrubber because it has limited commercial application

- Gold amalgamation for $\mathrm{Hg}$ control because it also has limited commercial experience

- Carbon injection for $\mathrm{Hg}$ control because of potentially causing a baghouse fire and was considered too inefficient

- Activated carbon because of low Hg loading when compared to the sulfur-impregnated carbon.

The WERF Feasibility Study Project Team has developed four practicable offgas treatment processes, which, if installed, would enable the WERF to meet the anticipated emission limits of the pending MACT rule. The offgas treatment processes under consideration are combinations of some of the various offgas treatment technologies described in Section 3.

Figure 5-1 exhibits four block flow diagrams for the offgas treatment options under consideration, which include the following:

- Option 1-Dry Sorbent Injection

- Option 2-Spray Dry Absorption

- Option 3-Wet Scrubber Located Downstream of Filtration

- Option 4-Wet Scrubber Located Upstream of Particulate Removal.

\subsection{Dry Sorbent Injection Process Description}

A dry acid-gas removal system that could be implemented at the WERF consists of the components listed below. These components would be installed downstream of the existing WERF SCC:

- Evaporative cooler (partial-quench)

- Dry sorbent injection 


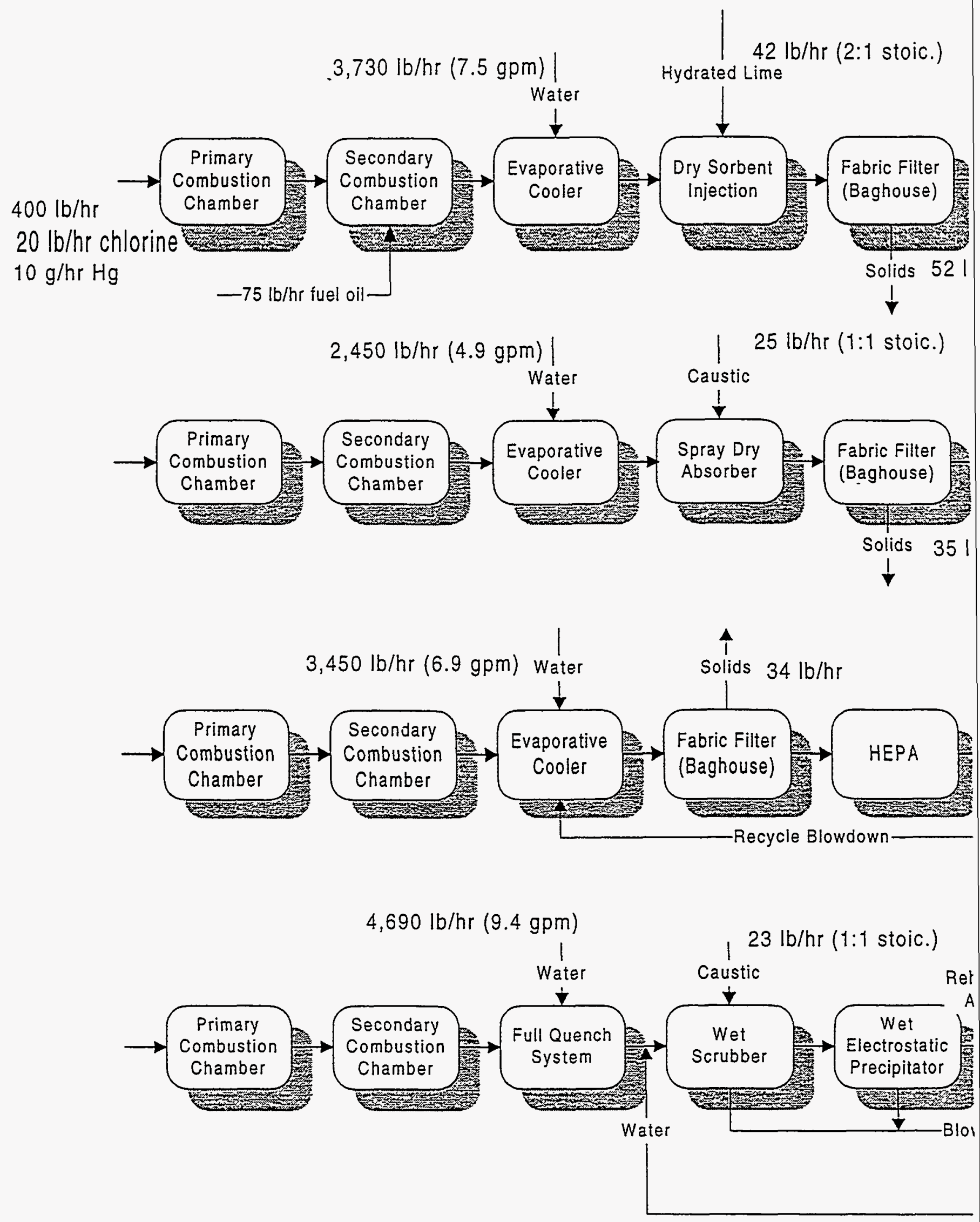

Figure 5-1. Proposed Air Pollution ( 
$2,730 \mathrm{acfm}$

Dilution Air

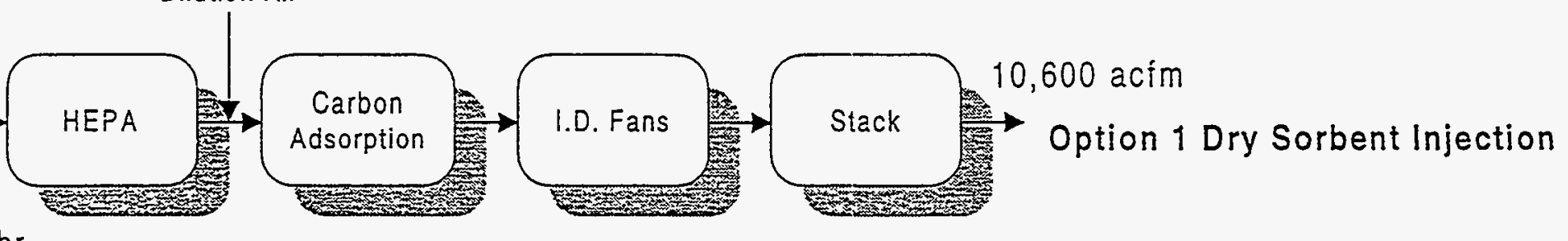

$3,580 \mathrm{acfm}$

Dilution Air

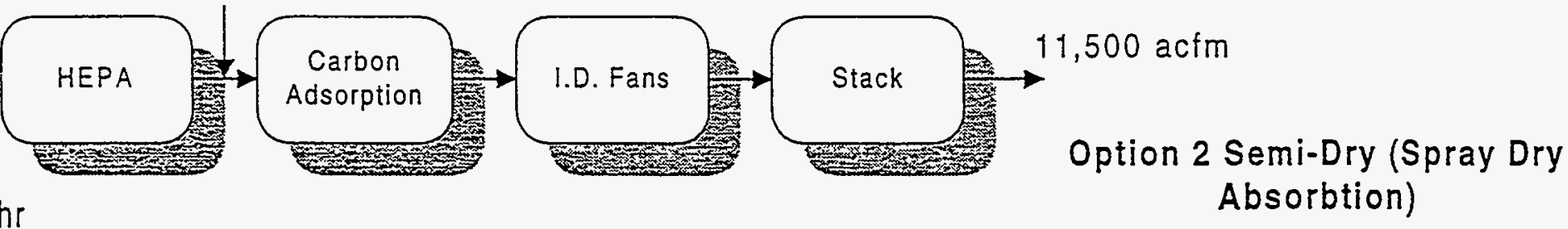

Caustic $24 \mathrm{lb} / \mathrm{hr}$ (1:1 stoic.)
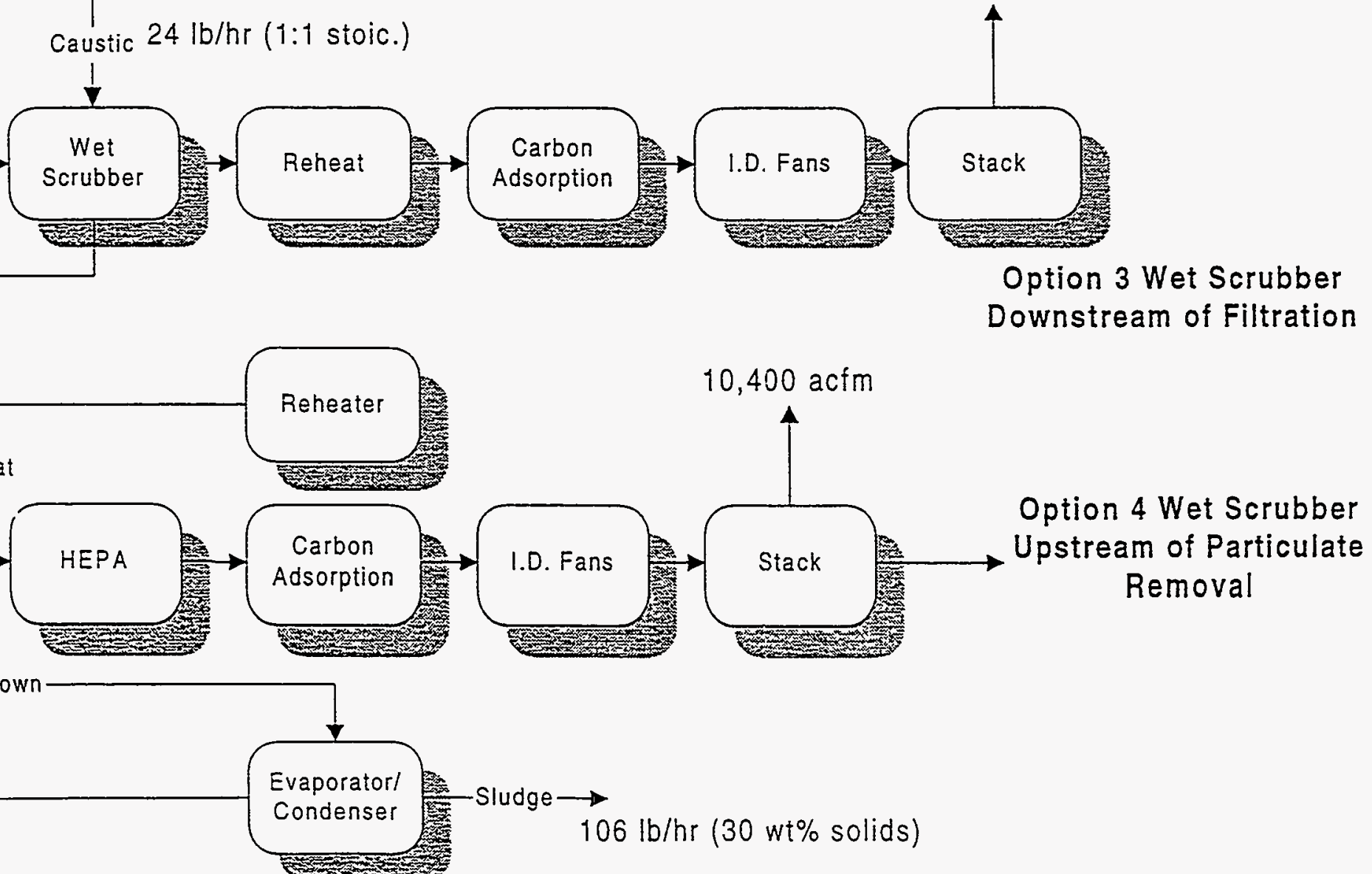

atrol System Options 
- Fabric filter baghouse

- HEPA filtration

- Carbon adsorber

- Induced draft fans and stack.

The following is a more detailed component and operation description for the dry acid-gas removal system that could be implemented at the WERF.

\subsubsection{Evaporative Cooler}

Off gas cooling can be accomplished in an evaporative cooler, which is a vertical cylindrical, refractorylined carbon steel vessel. Based upon the calculated offgas volumetric flowrates, an evaporator with an approximate outside diameter of $5 \mathrm{ft}$ and a height ranging from $20-25 \mathrm{ft}$ would be required. The hot offgas from the secondary chamber flows into the top of the quench vessel equipped with a gas flow distribution system that is approximately $6-7 \mathrm{ft}$ long. The distributor causes the offgas to flow downward with minimal swirling action. Water is added to the hot gas through dual fluid, air-atomizing nozzles that are located near the bottom of the gas distributor. The dual fluid nozzles use atomizing air to create finely dispersed water droplets (less than $800 \mu \mathrm{m}$ ), which create the surface area necessary for efficient heat transfer from the gas to the liquid water droplet. As the hot gas and water droplets flow downward through the quench vessel, the offgas is cooled and the water droplet disappears due to evaporation. This offgas cooling is effectively achieved within $0.5-1$ second after the gas enters the vessel.

Under maximum offgas temperature and flow conditions, preliminary design calculations indicate that $7.5 \mathrm{gpm}$ of water is required to control the offgas temperature exiting the quench vessel cooler to $350^{\circ} \mathrm{F}$. Adding water to the offgas increases its moisture content from 6 volume percent (vol\%) to $37 \mathrm{vol} \%$. It is important to maintain the offgas temperature above the dew point temperature to avoid condensation in the baghouse located downstream. The cooler is usually equipped with a bottom sump to collect any condensate that may form during an upset of operating conditions. This condensate would reevaporate once proper operating conditions are restored, or it may be possible to recycle the condensate back to the top of the evaporative cooler.

\subsubsection{Dry Sorbent Injection}

In a DSI process, a dry powder sorbent (i.e., an alkali reagent) is injected into the offgas at a point where the sorbent can react with the acid gases that are present. A common method of sorbent injection is via pneumatic transport through a nozzle, but gravity fed mechanical feeders, such as augers, are also used. In Option 1 the dry sorbent is added upstream of the baghouse allowing the sorbent to build-up a porous layer on the fabric filters in the baghouse. Under these conditions, the baghouse is a dry reactor chamber that effectively causes the offgas to flow through the reagent. This feature provides the direct contact between the acid gas and solid reagent material, which is necessary to neutralize the acid gas.

There are several alkali materials that could be used in DSI. The two most common reagents are hydrated lime and sodium bicarbonate. 
5.1.2.1 Calcium Hydroxide (Hydrated Lime). The chemistry for calcium hydroxide reacting with hydrochloric acid is shown in the following equation:

$\mathrm{Ca}(\mathrm{OH})_{2(\mathrm{~s})}+2 \mathrm{HCl}_{(\mathrm{g})} \rightarrow \mathrm{CaCl}_{2(\mathrm{~s})}+2 \mathrm{H}_{2} \mathrm{O}_{(\mathrm{g})}$

where:

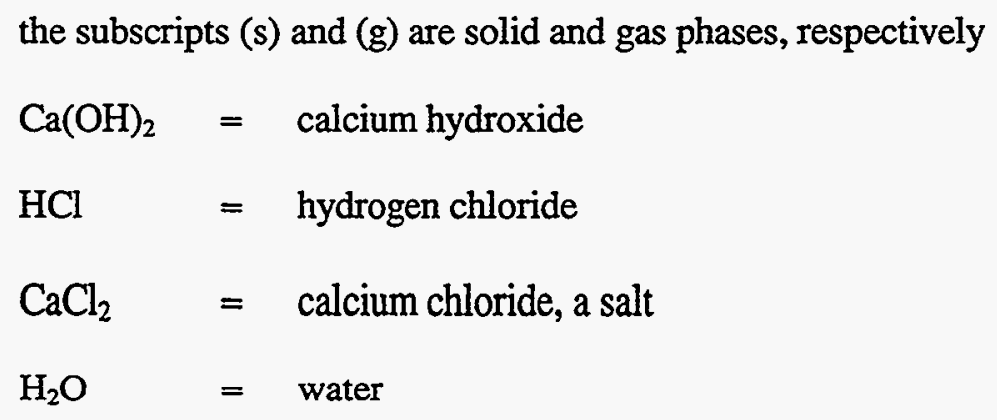

The extent of reaction is a function of the stoichiometric ratio of lime-to-HCl, the lime particle size, the acid gas concentration, and the offgas relative humidity. ${ }^{52}$ The above reaction is dependent upon the $\mathrm{HCl}$ in the gas phase contacting the solid hydrated lime. Some contact will occur where the hydrated lime is introduced into the offgas. However, most of the $\mathrm{HCl}$ will come in contact with the accumulated solid sorbent as the offgas passes across the filter bags where the solid cake has accumulated on the outer surface of the filter. ${ }^{52,53}$ The reaction occurs initially at the surface of the solid particles. Subsequent reaction on the particle cannot occur until the gas diffuses across the reaction product on the particle surface to unreacted material beneath it. Because not all of the lime can easily come in contact with the offgas, it is necessary to feed in excess of the stoichiometric feed rate (from 100 to 200\%) to achieve a desired acid gas removal efficiency. More complete alkali consumption is ensured when using the smaller hydrated lime particles, which provide greater surface area per sorbent mass for the gas-solid reaction.

In general, the process is effective when the gas stream is cooled in a range of $300-350^{\circ} \mathrm{F}$ and is partially saturated. It is important to keep the offgas temperature above the dew point and not let the gases become saturated because wet surfaces from condensation will cause problems, such as filtercake buildup and baghouse corrosion.

A potential operational problem is caused by the deliquescent nature of calcium chloride that results in the removal of moisture from the ambient gas surrounding it. This phenomenon occurs when the gas and solids temperature is less than the estimated dew point temperature of $266^{\circ} \mathrm{F}^{52}$ Liquids can accumulate in the baghouse and hopper presenting a material handling problem and increasing the potential for localized corrosion. If the moist material is present on the baghouse filter bags, blinding of the bags may occur when dried by the hot offgas. The WERF shut down procedure calls for ambient air to be drawn through the APCS so that the building air can be vented through the HEPA filter located downstream of the baghouse before it is sent to the exhaust stack. Building air will contain moisture that can be absorbed by calcium chloride. Since it is impossible to remove all the calcium chloride from the baghouse prior to shutdown, a by-pass duct with valving and blinds will be used during WERF shutdowns. The baghouse will be kept under negative pressure with minimal in-leakage to prevent liquid accumulation in the unit. 
5.1.2.2 Sodium bicarbonate. A second reagent under consideration is sodium bicarbonate, which decomposes to sodium carbonate upon heating.

$$
2 \mathrm{NaHCO}_{3(s)} \rightarrow \mathrm{Na}_{2} \mathrm{CO}_{3(\mathrm{~s})}+\mathrm{CO}_{2(\mathrm{~g})}+\mathrm{H}_{2} \mathrm{O}_{(\mathrm{g})}
$$

where:

$$
\begin{aligned}
& \mathrm{NaHCO}_{3}=\text { sodium bicarbonate } \\
& \mathrm{Na}_{2} \mathrm{CO}_{3}=\text { sodium carbonate } \\
& \mathrm{CO}_{2}=\text { carbon dioxide }
\end{aligned}
$$

As the decomposition products of water and carbon dioxide exit the sorbent particles, they expose fresh material surface of activated sodium carbonate to react with acid gases.

$\mathrm{Na}_{2} \mathrm{CO}_{3(\mathrm{~s})}+2 \mathrm{HCl}_{(\mathrm{g})} \rightarrow 2 \mathrm{NaCl}_{(\mathrm{s})}+\mathrm{CO}_{2(\mathrm{~g})}+\mathrm{H}_{2} \mathrm{O}_{(\mathrm{g})}$

where:

$\mathrm{NaCl} \quad=$ sodium chloride

The reaction of sodium carbonate and acid gas, as well as release of water and carbon dioxide during heating, ensures high surface area for the rapid reaction. In this way, virtually all of the sorbent used is consumed. The technology is similar, in principal, to that of dry lime injection technology in that acid gas removal is directly related to the contact time between the offgas and the dry particles.

Factors influencing acid gas removal efficiency include cake thickness on baghouse filter bags, reagent particle size, gas velocity, and the baghouse air-to-cloth ratio. $\mathrm{HCl}$ removal efficiencies ranging from $95-99 \%$ have been achieved. ${ }^{54,55}$ Higher efficiencies are associated with greater particulate feed rates. Stoichiometric ratios on the order of $150 \%$ may be required.

5.1.2.3 Comparison of Dry Injection Reagents. Advantages of the different sorbents are as follows: ${ }^{54}$

- For a given filter cake thickness, the porous nature of sodium bicarbonate particles during reaction results in lower pressure drop across fabric filter (for same cake thickness).

- Sodium bicarbonate particulates are inherently benign, unlike lime, which can react with moisture, such as an operator's perspiration, to produce a strong alkali.

- Sodium bicarbonate sorbent is less erosive than lime, resulting in lower maintenance costs associated with gas flow (eductor, filter bags).

- Sodium chloride produced from sodium bicarbonate will not become wet like the calcium chloride does during shutdown conditions, thereby reducing the potential to blind the baghouse filter cloths.

- In both cases, hydrated lime injection and sodium bicarbonate injection, the mixture of flyash, absorbent product, and excess sorbent have been stabilized to meet metal Toxicity 
Characteristic Leaching Procedure leaching requirements. However, lime is a more common waste stabilization reagent than sodium bicarbonate.

- Sodium bicarbonate costs are on the order of $\$ 300$ per ton compared to $\$ 75-\$ 100$ per ton of hydrated lime.

In general, dry reagents are not as reactive as wet alkali material and vendor information indicates that it is necessary to use excess alkali material. If hydrated lime is used, it is proposed that $100 \%$ excess would be required for this application. Assuming a $97.2 \% \mathrm{HCl}$ removal efficiency, a hydrated lime addition rate of $42 \mathrm{lbs} / \mathrm{hr}$ would allow for a continuous chlorine waste feed rate of $20 \mathrm{lbs} / \mathrm{hr}$. In the case of sodium bicarbonate, assuming a 70\% excess to achieve the same degree of $\mathrm{HCl}$ removal, $81 \mathrm{lbs} / \mathrm{hr}$ would be required for a chlorine waste feed rate of $20 \mathrm{lbs} / \mathrm{hr}$.

\subsubsection{Baghouse/Dust Transfer}

The existing baghouse system installed at the WERF consists of two baghouse compartments that are installed in parallel with a common outlet plenum. Each baghouse is designed to continuously filter $8,000 \mathrm{acfm}$ (at $400^{\circ} \mathrm{F}$ ) of offgas. The units are dust-tight compartments, each containing 144 filter bags. Each bag is $45 / 8$ in. in diameter and has a length of $100 \mathrm{in}$. (effective filtering length $=96 \mathrm{in}$.). Based upon the current operating conditions at WERF, the effective filtering area provides a 6:1 air-to-cloth ratio. When using a DSI system, an air to cloth ratio in the range of $2: 1$ or $3: 1$ is necessary. Eliminating the dilution air upstream of the baghouse in favor of an evaporative cooler reduces the offgas volumetric flow such that an air to cloth ratio of 3:1 can be obtained with the existing baghouse system.

However, the existing baghouse filtration system will require the following modifications to support the dry sorbent process:

- Insulation is required in both the hopper and tube sheet areas to minimize condensation

- Addition of hopper heaters or heat tracing

- Addition of a hopper vibrator

- Reconfiguration of the baghouse hoppers to make the walls steeper

- Redesign the flow path of the incoming offgas

- Bag replacement using new fabric material more compatible with DSI, such as Gore-Tex

- Modification to the dust transfer system to accommodate increased particulate loading (60 lbs/hr).

The solids exiting the baghouse bottom hoppers is pneumatically transferred to a container located in the lower level of the WERF facility. The dust transfer system has been problematic for the WERF operations due to plugging of solids in the transfer line. Adding dry sorbent will significantly add to the amount of solids that need to be removed from the baghouse. The baseline cost estimate includes a simple solids transfer system. It is proposed that the solids are gravity fed from the baghouse hoppers into containers located directly beneath them in the lower level of the facility. Rotary star valves will be used to control the solids flow from the hoppers. 


\subsubsection{HEPA Filtration}

The HEPA filtration system consists of a 304 stainless-steel housing, a HEPA filter rack holding an array of 16 HEPA filters, and a prefilter rack holding 16 prefilters. Nuclear-grade HEPA filters are constructed of acid-resistant media and stainless-steel frames. Industrial-grade prefilters with stainless-steel frames are installed upstream of the HEPA filters. The HEPA filtration system currently installed at the WERF is sufficient for DSI without modification.

\subsubsection{Carbon Adsorption}

As discussed in Section 4.2, several Hg control technologies were considered. Based upon these studies, sulfur-impregnated carbon seems to be the best alternative for Hg capture. Carbon injection was not considered as a viable method because of process safety considerations and the known inefficiencies associated with the process. Therefore, a fixed carbon bed was chosen for volatilized $\mathrm{Hg}$ control. An added benefit for using carbon adsorption is that most D/Fs will also be adsorbed by the carbon.

The sulfur-impregnated activated carbon suppliers indicate that $\mathrm{Hg}$ removal efficiencies improve with increasing offgas temperatures until volatilization of the impregnated sulfur occurs. The temperature at which the sulfur volatilizes from the activated carbon varies with manufacturer. One type of Sulfur-impregnated activated carbon has been identified that has minimal sulfur loss up to $200^{\circ} \mathrm{C}\left(390^{\circ} \mathrm{F}\right)$. This adsorbent has been successfully used at offgas temperatures as high as $250^{\circ} \mathrm{F}$.

Sulfur-impregnated activated carbon is typically placed in a fixed-bed configuration designed to ensure a maximum contact time between the offgas and carbon. To prevent the carbon beds from plugging, they are typically placed downstream of the particulate removal device (i.e., baghouse and HEPAs).

A second operating constraint is to have temperature of the offgas entering the carbon adsorber well above the offgas dew point temperature to avoid water condensation. Water condensate in the bed will decrease its adsorption capacity, and the gas flow differential pressure across the beds will increase. If this should occur, the carbon suppliers have stated that the performance efficiency of the carbon bed can be recovered by drying out the bed.

The carbon adsorption arrangement proposed for this system is a fixed bed process where the offgas will flow down through a single bed of sulfur-impregnated carbon. Prior to entry into the carbon bed, the offgas will be conditioned with approximately $3000 \mathrm{acfm}$ of dilution air to lower the offgas temperature to approximately $250^{\circ} \mathrm{F}$ and the dew point to about $130^{\circ} \mathrm{F}$. The source of the dilution air could be the building air (second dilution), which is currently added upstream of the baghouse.

Preliminary design calculations indicate that a $4 \mathrm{ft}$ thick carbon bed having a surface area of approximately $210 \mathrm{ft}^{2}$ is required to achieve the desired offgas residence time in the carbon bed. At these conditions, a minimum $\mathrm{Hg}$ removal efficiency of $99.4 \%$ is expected. The carbon adsorber bed has been sized to control $\mathrm{Hg}$ emissions based upon a continuous mercury waste feed rate of $10 \mathrm{~g} / \mathrm{hr}$ for $57,600 \mathrm{hrs}$ of WERF operations before the bed will need to be replaced. With this configuration and the design offgas volumetric flowrate, it is anticipated that the carbon bed will cause $10 \mathrm{in}$. of water column pressure loss. 


\subsubsection{Induced Draft Fans, Emergency Power, and Stack}

The induced draft fans currently installed at the WERF do not have the capacity for the APCS configurations being considered. The existing fans are rated for 20 in. of water column static pressure at $8,000 \mathrm{acfm}$ and $350^{\circ} \mathrm{F}$. Although a slightly reduced offgas temperature $\left(250^{\circ} \mathrm{F}\right)$ will increase the density of the offgas which consequently increases the fan static pressure rating. The increase is not adequate to compensate for the additional pressure drop associated with the proposed APCS modifications. The fans must be rated to accommodate the maximum anticipated system pressure drop that is based upon the following estimates:

- Carbon bed-10 in. of water column

- HEPA filters -5 in. of water column

- Prefilters -5 in. of water column

- Baghouse -8 in. of water column

- Evaporative cooler-2 in. of water column

- Miscellaneous system losses -5 in. of water column

- Contingency -5 in. of water column

- Total-40 in. of water column.

One of the induced draft fans is connected to an emergency power diesel generator to ensure that the APCS continues to operate during power outages. A review of indicates that the diesel generator has $82 \mathrm{~kW}$ of excess capacity available for incinerator operations. ${ }^{56}$ This equates to approximately $110 \mathrm{HP}$. The power requirement for the existing induced draft fan motor is $65 \mathrm{~kW}(87 \mathrm{HP})$.

\subsubsection{Continuous Emissions Monitors}

A beta gauge appears best suited for use as a continuous PM monitor. Its calibration depends so weakly on particle properties that it is considered universal and absolute; thus, eliminating the need for site-specific calibration against manual gravimetric measurements. A heated flame ionization detection instrument will be installed to determine the THC concentration.

\subsection{Spray Dry Absorber (Option 2)}

Option 2, as shown schematically in Figure 5-1, involves replacing the DSI system in Option 1 with an SDA. The SDA is commercially proven technology for semidry acid-gas removal.

An SDA semidry acid-gas removal system that could be implemented at the WERF consists of the components listed below. These components would be installed downstream of the existing SCC:

- Evaporative cooler

- Spray dry absorber

- Fabric filter baghouse 
- HEPA filtration

- Carbon adsorber

- Induced draft fans and stack.

The following section describes the critical design and operating features for the SDA. The remaining APCS components listed are similar to those described in Option 1 (Section 5.1).

\subsubsection{Spray Dryer Absorber}

Spray dryer absorbers are typically configured as cylindrical vessels. Water and alkali reagents are mixed to form a solution that is injected into the reactor vessel as a finely atomized spray produced by dual fluid nozzles or mechanical rotary atomizers. The caustic mixture is sprayed into the top of the SDA to flow co-currently with the offgas. The offgas enters the reactor vessel where the acid gases are absorbed into the small liquid droplets and react with the alkali reagent to form a salt. As the droplets pass through the reactor, heat from the offgas evaporates the water, cooling the offgas and forming solid particles of salt, unreacted alkali, and flyash. Acid gas in the offgas continues to react with the dry alkali. The dry solids are then collected downstream of the reactor in the baghouse. To allow for complete moisture evaporation and maximize acid conversion, offgas residence times in the SDA vessel range from 4-20 seconds.

The SDA considered for the WERF is based on an existing vessel design. The reactor vessel for the WERF SDA has been installed and is sized to accept a gas flowrate of 13,179 acfm at $750^{\circ} \mathrm{F}$, while maintaining a gas residence time of 18 seconds. The original gas flowrate design was based on processing both the first and second dilution air streams currently used at WERF to cool the offgas, but without using the heat exchanger. Operating the SDA under these conditions would promote $\mathrm{D} / \mathrm{F}$ formation similar to the existing three-step cooling process. Additionally, using large dilution airflows to cool the offgas makes carbon adsorption treatment expensive because more surface area is required for a given gas residence time. In order to mitigate these problems, an evaporative cooler located between the secondary chamber and the SDA is part of this option.

Consistent with the existing SDA design, sodium hydroxide would most likely be used as the alkali reagent. Although slaked lime can be used instead, the slaking operation can be untidy and troublesome to operate especially in the winter. The design calculations show that when processing $20 \mathrm{lbs} / \mathrm{hr}$ of $\mathrm{HCl}$ in the offgas, $45 \mathrm{lbs} / \mathrm{hr}$ of $\mathrm{NaOH}$ mixed with water will be required to achieve $99 \% \mathrm{HCl}$ removal.

The main advantage for using the SDA versus DSI is that the SDA neutralizes acid gas more efficiently than the dry system. The disadvantage is the difficulty of operating the system in the proper conditions to control droplet size. If the particles are too small, they will dry out before sufficient absorption can take place. If the particles are too large, there is less surface area per volume, and gas mixing is reduced. The droplet size also affects the size of solid particles that are produced. If the droplets are too small, a very fine particulate is produced and particulate removal is difficult. Large particles may result in insufficient evaporation and may actually lead to a coating or buildup inside the reactor vessel. 


\subsection{Wet Scrubber Downstream of Particulate Filtration (Option 3)}

Option 3 is schematically shown in Figure 5-1. Since the proposed MACT emission standard for $\mathrm{HCl}$ is based upon wet scrubber technology, Option 3 was investigated for implementation at the WERF. The Option 3 APCS consists of the components listed below and would be installed in the order listed downstream of the existing SCC:

- Evaporative cooler

- Fabric filter baghouse

- HEPA filtration

- $\quad$ Packed bed scrubber with saturator

- Off gas reheater with dilution air addition

- Carbon adsorber

- Induced draft fan and stack.

The descriptions for the evaporative cooler, baghouse, HEPA filter, and carbon adsorber are similar to those listed under Option 1, Section 5.1. The induced draft fans for this option would require slightly more horsepower for the extra pressure drop over the wet scrubber and gas reheater.

\subsubsection{Packed Bed Scrubber}

The packed bed scrubber is designed to remove acid gases (typically hydrochloric acid and sulfur dioxide) from the offgas by contacting them with a caustic scrub solution. The solution $\mathrm{pH}$ is maintained between 8 and 10 to prevent the absorption of carbon dioxide into solution. When operating in this $\mathrm{pH}$ range, there is a risk that oxidized $\mathrm{Hg}$ will be converted back to the more volatile element $\mathrm{Hg}$.

To prevent the accumulation of the salt products in the scrubber solution, a portion of the solution needs to be withdrawn (blow-down) and replaced with fresh make-up scrubber solution. The purged scrubber solution can be treated by evaporating the excess water and stabilizing the resulting sludge for disposal, or it can be recycled back to the evaporative cooler. However, evaporating or recycling the blow-down could re-volatilize the low boiling point contaminants including mercury. Under blow-down recycle conditions, the volatile metals could accumulate within the APCS to such a degree that either carryover of the metals from the wet scrubber will occur or the wet scrubber solution will need to be replaced. The remaining salts, including sodium chloride, in the blow-down will be dried in the evaporative cooler and collected in the baghouse.

\subsubsection{Gas Reheater}

Since the offgas exiting the wet scrubber is saturated, it must be reheated before it enters the carbon adsorber. In order to avoid D/F reformation associated with direct-fired heating of the offgas, a separate dilution air stream is heated in a direct-fired heater and added to the offgas. 


\subsection{Wet Scrubber Upstream of Particulate Removal (Option 4)}

Option 4 represents the state-of-the-art APCS, which is a wet scrubbing system with zero liquid discharge. Option 4, as shown in Figure 5-1, is an APCS that uses a wet scrubber located upstream of the particulate control mechanism. In this case, the baghouse is replaced with a wet electrostatic precipitator, and the wet scrubber design includes the ability to process offgas containing PM.

The Option 4 APCS consists of the components listed below; they would be installed in the order listed downstream of WERF existing SCC:

- Full-quench evaporative cooler

- Venturi wet scrubber

- Wet electrostatic precipitator

- Evaporator

- Condenser

- Off gas reheater with dilution air addition

- HEPA filtration

- Carbon adsorber

- Induced draft fan and stack.

The technologies listed above that have not been previously described are detailed below.

\subsubsection{Full-Quench Evaporative Cooler}

A full-quench evaporative cooler is designed to cool the offgas to a completely saturated state. This is accomplished by introducing an atomized spray of water co-currently into the offgas exiting the SCC. In addition, excess water introduced into the evaporator as an atomized spray or along a wetted wall to ensure the offgas is cooled to its saturation temperature. Unlike in a partial-quench evaporative cooler, it is not undesirable if water is entrained in the offgas.

\subsubsection{Venturi Wet Scrubber}

A venturi wet scrubber is designed to bring into intimate contact a gas and liquid stream to remove particulate greater than 1 micron in diameter with high efficiency. In addition, by controlling the solution $\mathrm{pH}$, acid gases can be removed with high efficiency. These functions are achieved at the expense of a large pressure drop (on the order of $30 \mathrm{in}$. water column) across the venturi scrubber.

\subsubsection{Wet Electrostatic Precipitator}

In a wet electrostatic precipitator, a strong electric field is maintained between high-voltage discharge electrodes and grounded collecting electrodes. The discharge electrodes are made of wires or rigid frames, and the collecting electrodes are plates. A corona discharge from the discharge anode ionizes the gas passing through the precipitator, and the gas ions subsequently ionize the undesired 
particles. The negatively charged particles are then captured on the collecting electrode. Wet electrostatic precipitators use a water spray to remove the captured particulate. Wet electrostatic precipitators are useful for removing gaseous fumes and mists, as well as fine particulate. In Option 4, the wet electrostatic precipitator would remove fumes and mist from the offgas exiting the venturi wet scrubber.

The liquid collected in the bottom of the wet electrostatic precipitator is reused by pumping the solution back to the top. A small bleed stream must be removed and replenished with fresh water to prevent solids build-up within the unit. The bleed stream is evaporated to recover the solids as waste. The bleed stream cannot be sent back to the evaporative cooler like in Option 3 because the volatile metals will simply re-volatilize and flow back through the wet scrubber and wet electrostatic precipitator. The volatile metals would eventually accumulate within the liquid phase and carry-over with the offgas into the downstream HEPA filter and carbon adsorber and potentially out of the stack.

\subsubsection{Evaporator}

There are several evaporator designs including forced-circulation and falling film evaporators, which could be used for removing the excess water from the wet scrubber and wet electrostatic precipitator blow-down streams. The primary disadvantage for using an evaporator to remove the water to such a degree that the dissolved solids form a dry product is the complexity of the process. Typically force-circulation evaporators have a heat exchanger with steam flowing through the shell side and the fluid being heated flowing through the tube side. The heated liquid is sent to a vessel where some of the water flashes into steam, which is sent to HEPA filtration along with heated dilution air to avoid condensation and subsequent plugging of the filters. The liquid phase is recycled back to the heat exchanger along with fresh make-up (blow-down feed) and reheated. As the solids within the liquid phase increase, the boiling temperature and associated vapor pressure of the solution increases due to increasing solids concentration. The viscous concentrated liquor is re-circulated at high flowrates and system pressures to avoid scale formation on the heat transfer surfaces. Periodically, part of the liquor is removed from the evaporator, and it solidifies upon cooling.

\subsubsection{Condenser}

In order to prevent moisture condensation in the saturated offgas downstream of the venturi scrubber, water is removed from the offgas by cooling the offgas. The resulting condensate is recycled back to the venturi scrubber to make up for particulate-ladden blowndown sent to the evaporator for concentration.

\subsection{Selection of Recommended Air Pollution Control System} following:

The APCS, described in Section 5 as Options 1,2, 3 and 4, were each assessed in terms of the

- Technical feasibility

- Relative cost

- Physical requirements

- Impact on the WERF WAC. 
The evaluation was performed by the MACT Feasibility Task Team, which included WERF engineering, WERF Burn Plan Engineer, and chemical/mechanical process engineers assigned to this study. Collectively, the team members are very knowledgeable of the APC technologies that are commercially available for treating offgas produced by mixed waste incineration.

\subsubsection{Technical Feasibility}

Each APCS under consideration was evaluated in terms of its potential ability to meet the proposed MACT standards for $\mathrm{HCl}, \mathrm{Hg}$, and $\mathrm{D} / \mathrm{F}$ emissions. This assessment was based upon literature information and published historical data (i.e., track record) for the various APC devices that make up each system. Technical feasibility also includes effectiveness. Although all the APC options under consideration will meet or exceed the MACT rule, some systems will operate more efficiently than the others. When considering efficiency, the complexity, type, and number of equipment items were taken into account, along with the amount of material that needs to be handled.

\subsubsection{Physical Requirements}

This evaluation category includes the infrastructure and environmental conditions necessary to install and operate each APCS under consideration. The required floor space or footprint for each system along with its utility requirements were taken into account during the evaluation process. Although all the APCSs under consideration can be installed within the existing facilities and utility limits at WERF, this criterion was included in the evaluation process to ensure that it was taken into account.

\subsubsection{Impact on the WERF Waste Acceptance Criteria}

Each APCS is designed to improve the control for $\mathrm{HCl}, \mathrm{Hg}$, and D/F emissions. Some APCSs can accomplish this control with more restrictive waste feed limits for these constituents than is currently in the WERF WAC. Although none of the APCSs under consideration would unduly restrict the WERF WAC after the MACT rule is implemented, this criterion was included in the evaluation process to ensure that it was considered.

\subsubsection{Relative Cost}

To evaluate the capital cost, order-of-magnitude equipment costs were used because preliminary project cost estimates for each option would require more than the budgeted engineering and design time necessary to develop such cost estimates. This strategy is acceptable given that the total equipment cost for each option is relatively distinct from any of the others. A preliminary budget authorization-type cost estimate was made for the selected APCS option and is discussed in Section 6.

The order-of-magnitude equipment cost estimates that were used to evaluate the capital costs for each APCS option are listed in Table 5-1. These equipment costs do not include freight or installation expenses and are based upon historical equipment cost data and minimal process knowledge. The accuracy for these estimates may exceed $\pm 30 \%$, but are considered sufficient for the technology decision process. Some of the equipment costs were calculated using the $7 / 10$ rule. This involves estimating the cost of an item based upon the estimated cost of a similar piece of equipment with a correction for throughput, size, or power. In the case of equipment processing a flowrate twice as great as equipment costing $\$ 10,000$, the cost of the larger equipment is estimated to be $\$ 10,000(2 / 1)^{0.7}$ or $\$ 16,250$. All equipment costs were corrected to first quarter, 1998 costs using the average Marshall \& Swift Equipment Cost Index for process industries. 


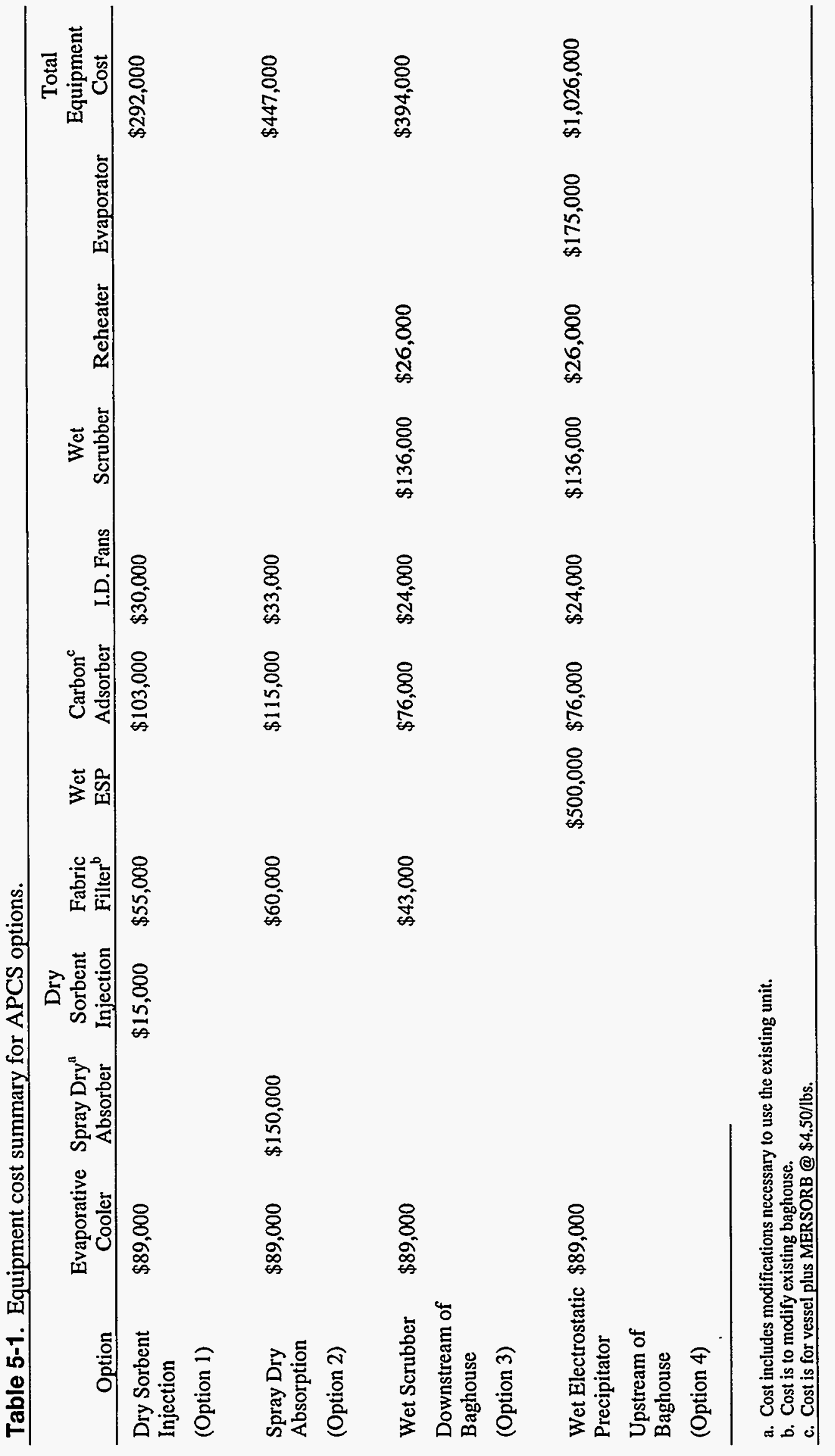




\subsubsection{Decision Plus Results}

A computer software program called Criterium Decision Plus was used to organize the APCS selection process and document the results. The software is designed to provide quantitative results to a qualitative decision-making process. Figure 5-2, a chart developed by Decision Plus, shows the technology evaluation criteria hierarchy along with their respective significance or weight factors. These factors, as assigned by a consensus of the team members, were used to calculate a rating score for each APCS option.

Each criterion listed above was subdivided into second level criteria, which were also assigned relative weight factors by the team. The only second level evaluation criterion that required further classification was Operating Cost, which was divided into third and fourth level criteria. Please note that the weight factors for the first level criteria add up to unity, and the lower tier criteria sum to the value assigned to the corresponding next higher level parameter. For example, technical feasibility with a weight of 0.415 means that when evaluating the APCS options, this criterion makes ups $41.5 \%$ of the total score. Technical feasibility was further subdivided into track record and performance. The team then compared track record to performance using the following sentence: "With respect to Technical Feasibility, on a scale measuring Importance and Ranging from Absolutely Better to Equal, Track Record rates Weakly Better than Performance." Criterium Decision Plus then assigns a weight factor of $31.1 \%$ to track record and $10.4 \%$ to performance, which sum up to $41.5 \%$.

After establishing the weight factors for the sub-criteria, each APCS option was rated by comparing it to the other three in a pair-wise fashion using the lowest sub-level criteria. Table 5-2 lists the lowest level criteria scores for each option. (Note: When all the options for a given criterion have a rating of 0.25 , this means they were all rated equal for that criteria.) The criteria with the most influence on the outcome include the following:

- $\quad$ Capital cost (0.215)

- $\quad$ Performance (0.104)

- Track record $(0.311)$ or commercially proven technology.

Ease of implementation ( 0.126 ) or constructability also has a significant weight assigned. However, all the options considered were judged to be similar for evaluating this criterion. The composite score for each option is the sum of the multiplication products of the criteria weight factor and the respective score each option received for the criteria. Options 1 and 4 have the highest composite score of 32\%, and Options 2 and 3 have scores of $12.7 \%$ and $23.9 \%$, respectively. Option 1 has the highest individual scores for lowest capital cost $(0.68)$, secondary waste issues $(0.69)$, manpower requirements $(0.58)$, and simplicity $(0.57)$.

Secondary waste issues include quantity, type, and difficulty to treat and dispose. Option 4 is rated highest in reliability (0.38), performance (0.49), and track record (0.57), which indicates that Option 4 has the highest rating for technical feasibility. These results are as expected. Option 4 is the state-of-the-art facility for APC and is technically superior to Option 1. However, after adding the costs for equipment installation, utility and facility modifications, engineering and construction, the total project cost would considerably exceed the $\$ 5 \mathrm{M}$ limit for General Plant Project funding. Therefore, Option 1 is considered to be the preferred alternative because it can be implemented for less than $\$ 5 \mathrm{M}$ while meeting the technical objectives for the project. 


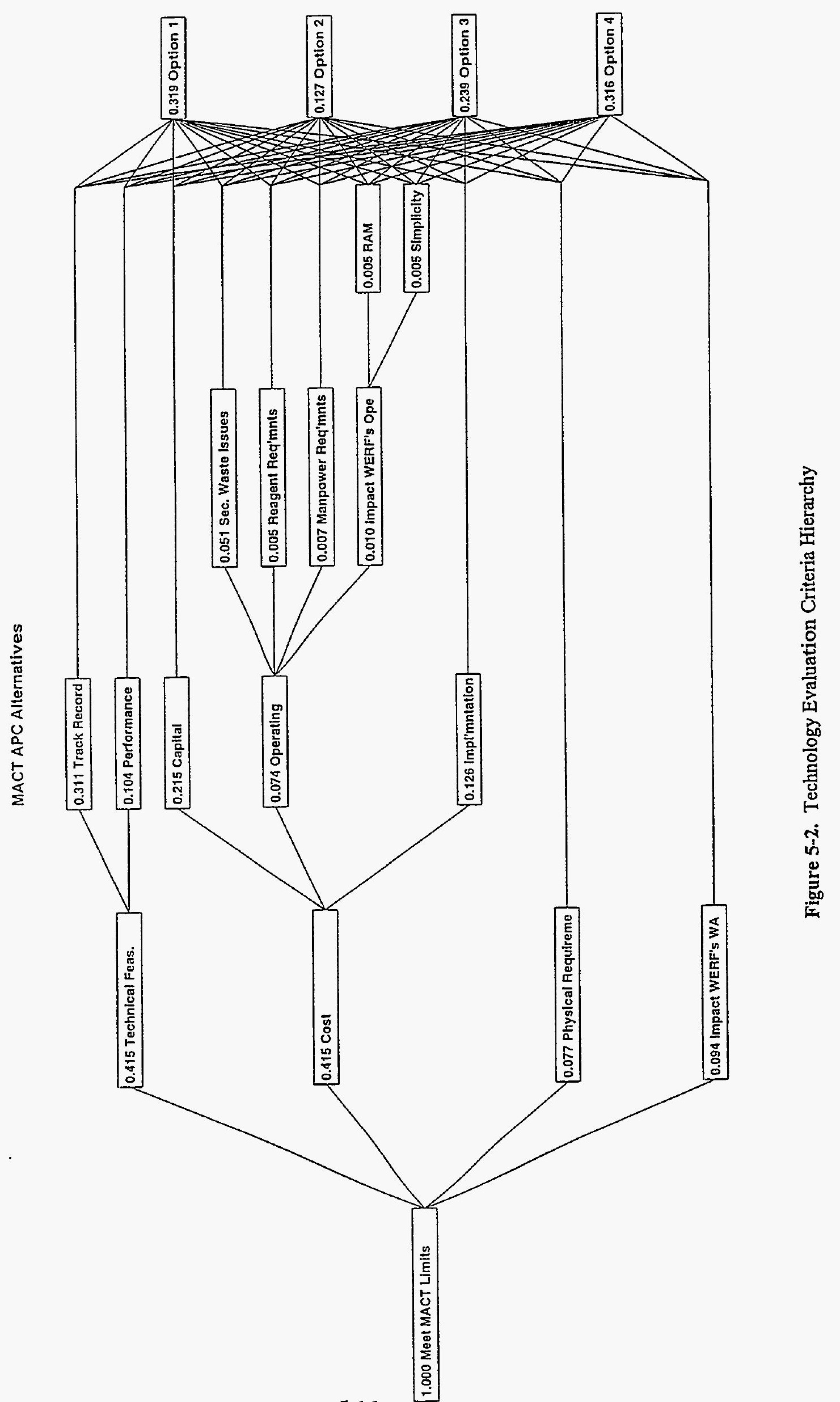


Table 5-2. Criterium decision plus scores.

Criteria

Lowest Level Criteria

Weight Factor Option 1 Option 2 Option 3 Option 4

Impact on the WERF WAC

0.094

0.25

0.25

0.25

0.25

Capital

0.215

0.68

0.09

0.2

0.03

Secondary Waste Issues

0.051

0.69

0.09

0.17

0.06

Reagent Requirements

0.005

0.1

0.3

0.3

0.3

Manpower Requirements

0.007

0.58

0.17

0.17

0.08

Reliability, Availability, Maintainability

0.005

0.13

0.13

0.38

0.38

Simplicity

0.005

0.57

0.14

0.14

0.14

Implementation (Retrofit)

0.126

0.25

0.25

0.25

0.25

Performance

0.104

0.11

0.11

0.3

0.49

Track Record

0.311

0.14

0.05

0.25

0.57

Physical Requirements

0.077

0.25

0.25

0.25

0.25

Results

0.32

0.13

0.24

0.32

\subsubsection{Technical Background for Option 1}

After choosing DSI (Option 1) as the preferred alternative for this study, site trips to facilities using this offgas treatment process were taken. The two facilities visited had incinerators similar in design to the WERF system. Both units were designed to treat medical waste and were equipped with evaporative coolers followed by DSI system using hydrated lime. Neither system was equipped for $\mathrm{Hg}$ control.

Based upon trial burn data, each facility is able to remove $96-98 \%$ of the waste feed chlorine as calcium chloride in the baghouse solids stream. Additional information concerning observations made during the site visits is located in Appendix E.

As mentioned previously, all the options considered would use fixed-carbon bed adsorbers for $\mathrm{Hg}$ control. Therefore, this technology was not a deciding factor when choosing which APCS option to recommend. However, it should be noted that $\mathrm{Hg}$ control is a developing technology. Of the $\mathrm{Hg}$ control technologies considered, sulfur-impregnated carbon adsorption has the least technical risk. There is minimal commercial operating data available to confirm the vendor information provided by Nucon. Thus, the design proposed here is conservative with respect to gas residence time and operating temperature. If the vendor information is correct, then $\mathrm{Hg}$ emissions should be well below the proposed MACT limit. 


\section{PLANNING COST ESTIMATE FOR RECOMMENDED AIR POLLUTION CONTROL SYSTEM OPTION}

A planning cost estimate was made for designing and installing offgas treatment Option 1 at the WERF. The total estimated cost for implementing the recommended APCS option is $\$ 4.2 \mathrm{M}$. Since the cost estimate is based upon preliminary information, it includes a $24 \%$ contingency. Planning cost estimate details are located in Appendix $\mathrm{C}$.

Preliminary equipment sizing was performed by the feasibility study team using the material and energy balance information as described in Section 4.6. Vendors were consulted for equipment footprint and utility requirements. The basis for the cost estimate includes the design, procurement, and installation of a new offgas cooler, a DSI system with reagent storage, a fabric filter baghouse, a carbon adsorber, and two offgas induced draft exhaust fans. The following sections summarize the costs associated with these activities. An additional section has been included to address the costs associated with operating Option 1.

\subsection{Capital Cost}

Figure 6-1 is a process flow diagram for the recommended WERF MACT upgrade. The flow diagram shows the major equipment items that must be designed, fabricated, and installed within the confines of the existing facility. The offgas line tie points for the new APCS begin with the line exiting the secondary chamber and end with the new induced draft fans connected to the north stack. The existing HEPA filter and one of the existing induced draft fans will be reused. However, they need to be relocated.

Cost estimates were solicited from equipment suppliers and vendors for the major equipment items. Vendors were also consulted for equipment and utility requirements. This effort includes prices for an evaporative cooler, a DSI system, a baghouse, a carbon adsorber, and two induced draft exhaust fans. Table 6-1 summarizes the capital cost for these items.

As seen in Figure 6-1, a new baghouse was included in the cost estimate because the required modifications to the existing baghouse may be too expensive and hazardous to accomplish. In addition, the air-to-cloth ratio for the existing baghouse is considered to be marginal for an efficient DSI system. The final decision concerning baghouse replacement versus modifying the existing one will be made during the detail design phase of the project.

\subsection{Design and Construction Costs}

Figures 6-2, 6-3, and 6-4 are preliminary three-dimensional drawings which conceptually show where the equipment could be installed within the confines of the WERF facility.

These drawings were used as visual aids to estimate the materials and labor required to install the equipment. Unit material costs were used for the ductwork and piping. The labor to install the equipment is based upon good engineering judgement. Man-hours to install the remaining mechanical and electrical equipment are based upon good cost estimate practices, including historical cost information when available. 


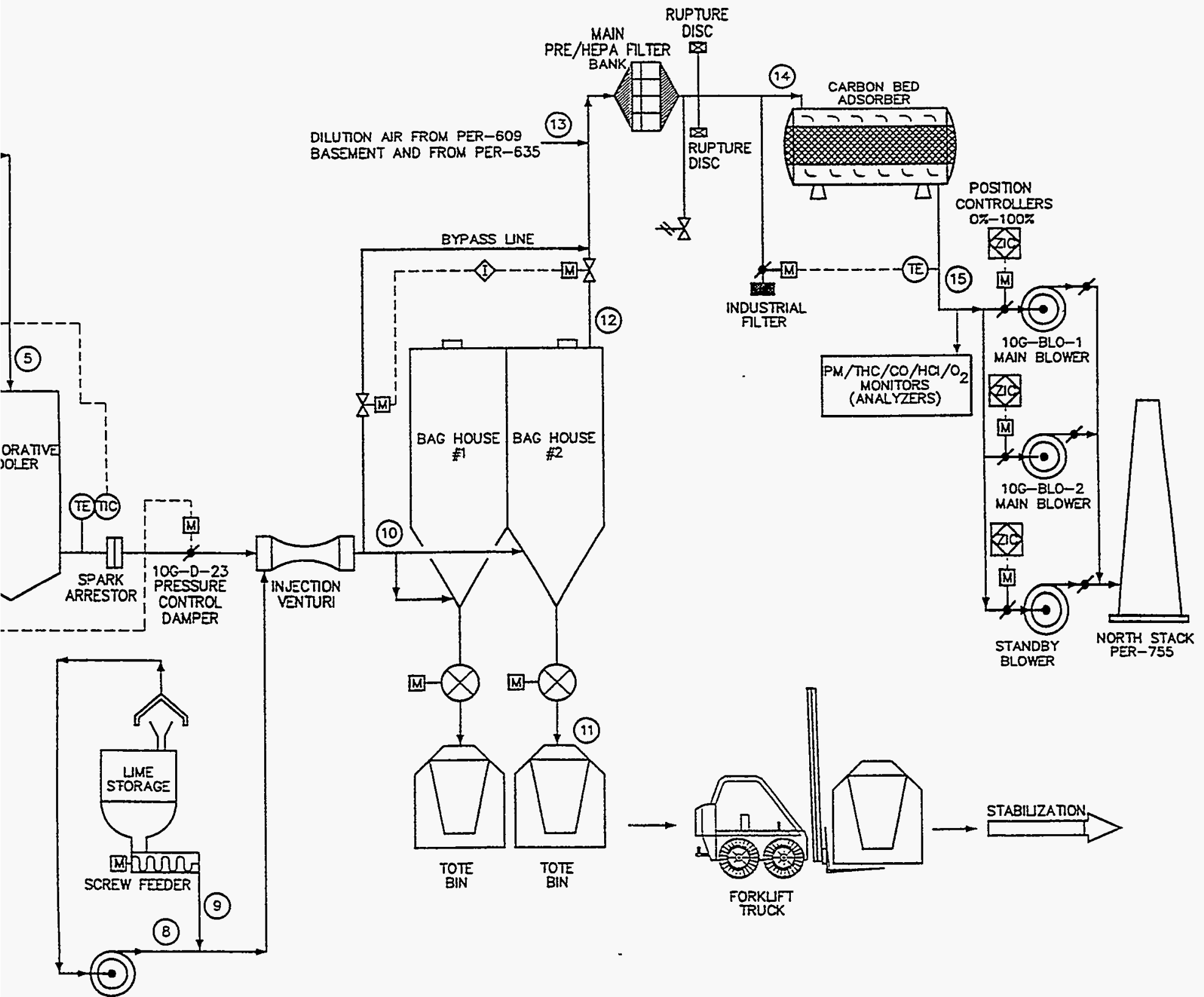

OSI BLOHER

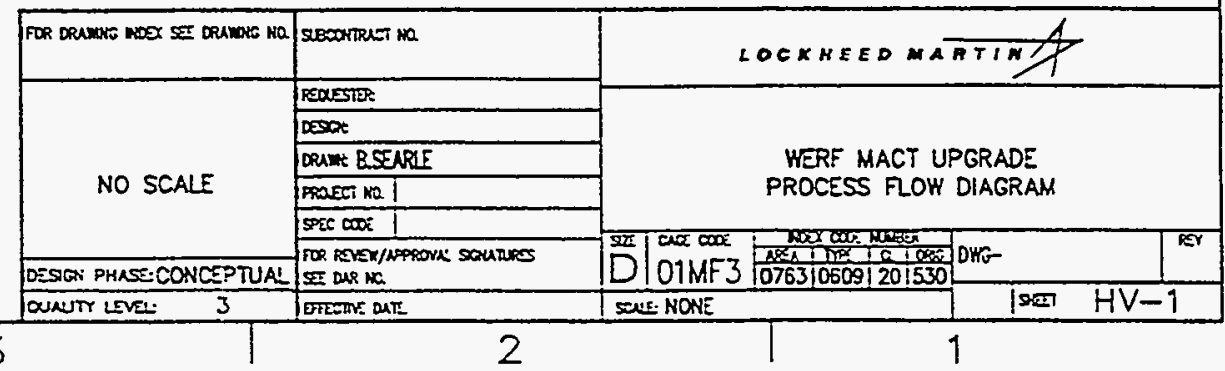




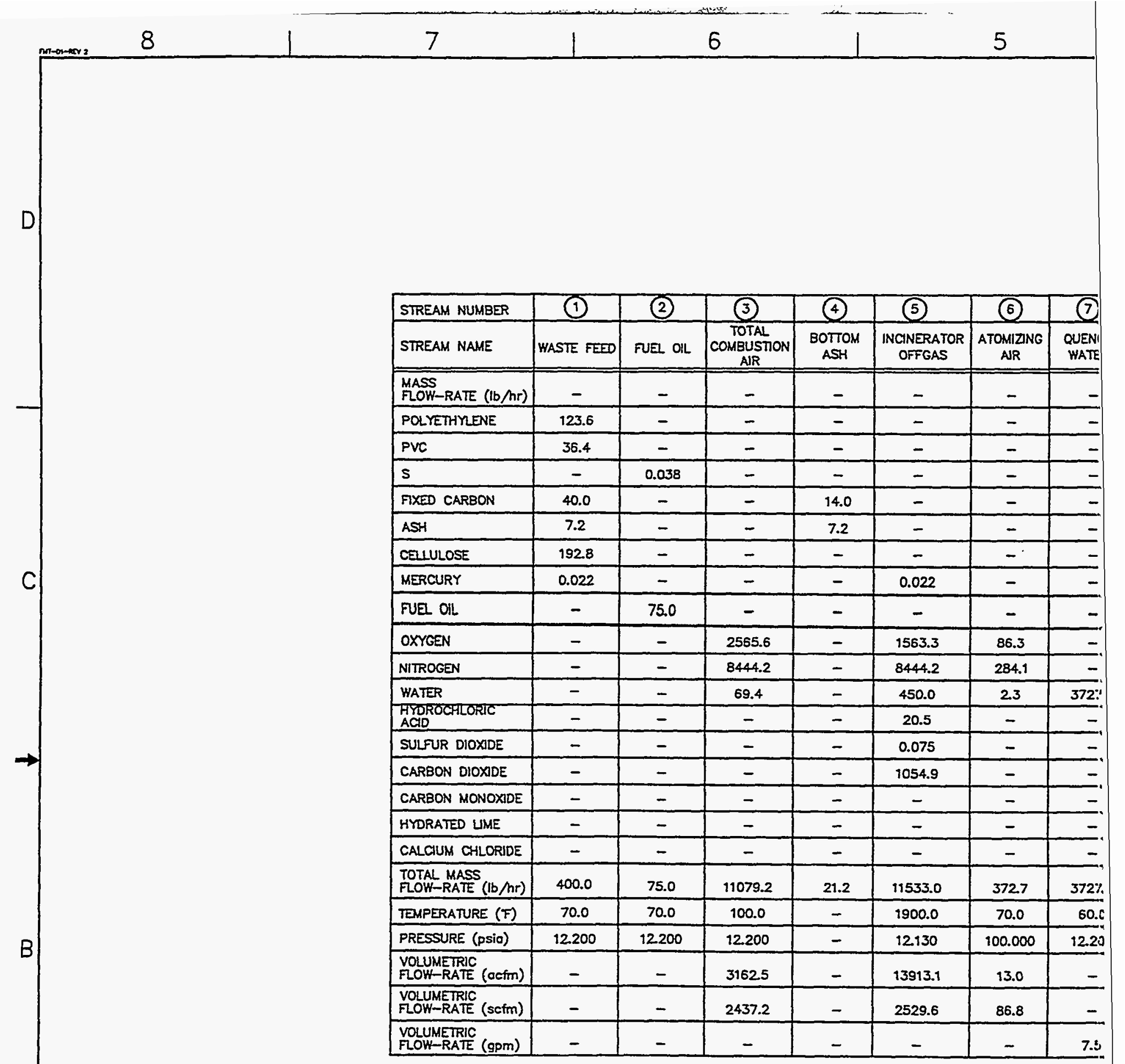

Figure 6-1. Continu 


\begin{tabular}{|c|c|c|c|c|c|c|c|}
\hline (B) & (9) & (10) & (11) & (12) & (13) & (14) & (15) \\
\hline $\begin{array}{c}\text { PNEUMATIC } \\
\text { AIR }\end{array}$ & $\begin{array}{c}\text { DRY } \\
\text { ABSORBENT }\end{array}$ & $\begin{array}{l}\text { TOTAL GAS } \\
\text { INTO } \\
\text { BAGHOUSE } \\
\end{array}$ & $\begin{array}{l}\text { TOTAL } \\
\text { BAGOUSE } \\
\text { SOUDS }\end{array}$ & $\begin{array}{l}\text { BAGHOUSE } \\
\text { OFFGAS }\end{array}$ & $\begin{array}{l}\text { DILUTION } \\
\text { AUR }\end{array}$ & $\begin{array}{l}\text { TOTAL GAS } \\
\text { INTO } \\
\text { CARBON BED }\end{array}$ & $\begin{array}{l}\text { STACK } \\
\text { OFFGAS }\end{array}$ \\
\hline - & - & - & - & - & - & - & - \\
\hline- & - & - & - & - & - & - & - \\
\hline- & - & - & - & - & - & - & - \\
\hline- & - & - & - & - & - & - & - \\
\hline - & - & - & - & - & - & - & - \\
\hline - & - & - & - & - & - & - & - \\
\hline- & - & - & - & - & - & - & - \\
\hline- & - & 0.022 & - & 0.022 & - & 0.022 & 0.0001 \\
\hline - & - & - & - & - & - & - & - \\
\hline 110.5 & - & 1760.2 & - & 1760.2 & 2214.7 & 3974.9 & 3974.9 \\
\hline 363.6 & - & 90924 & - & 9092.4 & 7289.3 & 16381.7 & 16381.7 \\
\hline 3.0 & - & 4182.4 & - & 4192.2 & 59.9 & 4252.1 & 4252.2 \\
\hline- & - & 20.5 & - & 0.6 & - & 0.6 & 0.6 \\
\hline - & - & 0.075 & - & 0.075 & - & 0.075 & 0.075 \\
\hline - & - & 1054.9 & - & 1054.9 & - & 1054.9 & 1054.9 \\
\hline- & - & - & - & - & - & - & - \\
\hline - & 41.8 & 41.8 & 21.5 & - & - & - & - \\
\hline- & - & - & 30.4 & - & - & - & - \\
\hline 477.1 & 41.8 & 16110.5 & 51.9 & 16100.4 & 9563.9 & 25664.3 & 25664.3 \\
\hline 70.0 & 70.0 & 340.5 & 322.6 & 3226 & 100.0 & 250.0 & 225.0 \\
\hline 100.000 & 12.200 & 12.060 & 11.770 & 11.770 & 12.200 & 11.770 & 11.230 \\
\hline 15.7 & - & 7545.0 & - & 7557.5 & 2730.0 & 10444.0 & 10560.0 \\
\hline 104.8 & - & 4021.0 & - & 4020.7 & 2103.9 & 6124.5 & 6124.1 \\
\hline - & - & - & - & - & - & - & - \\
\hline
\end{tabular}

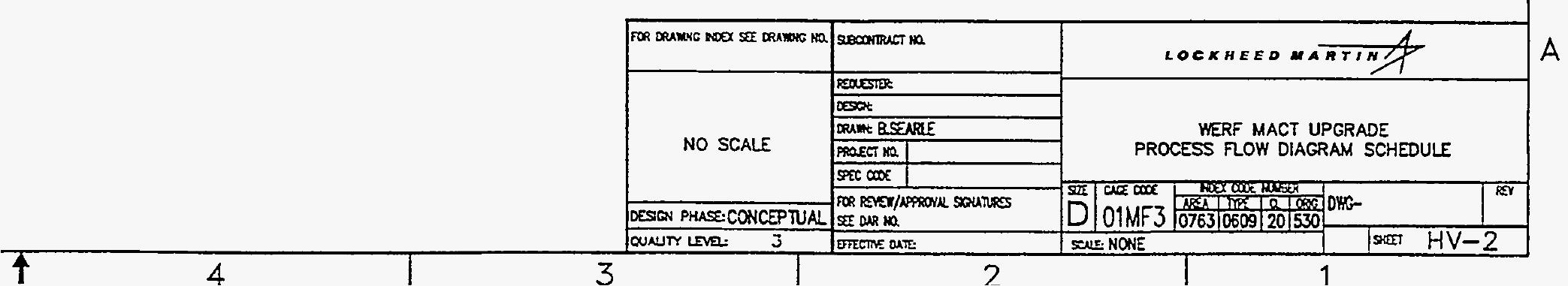




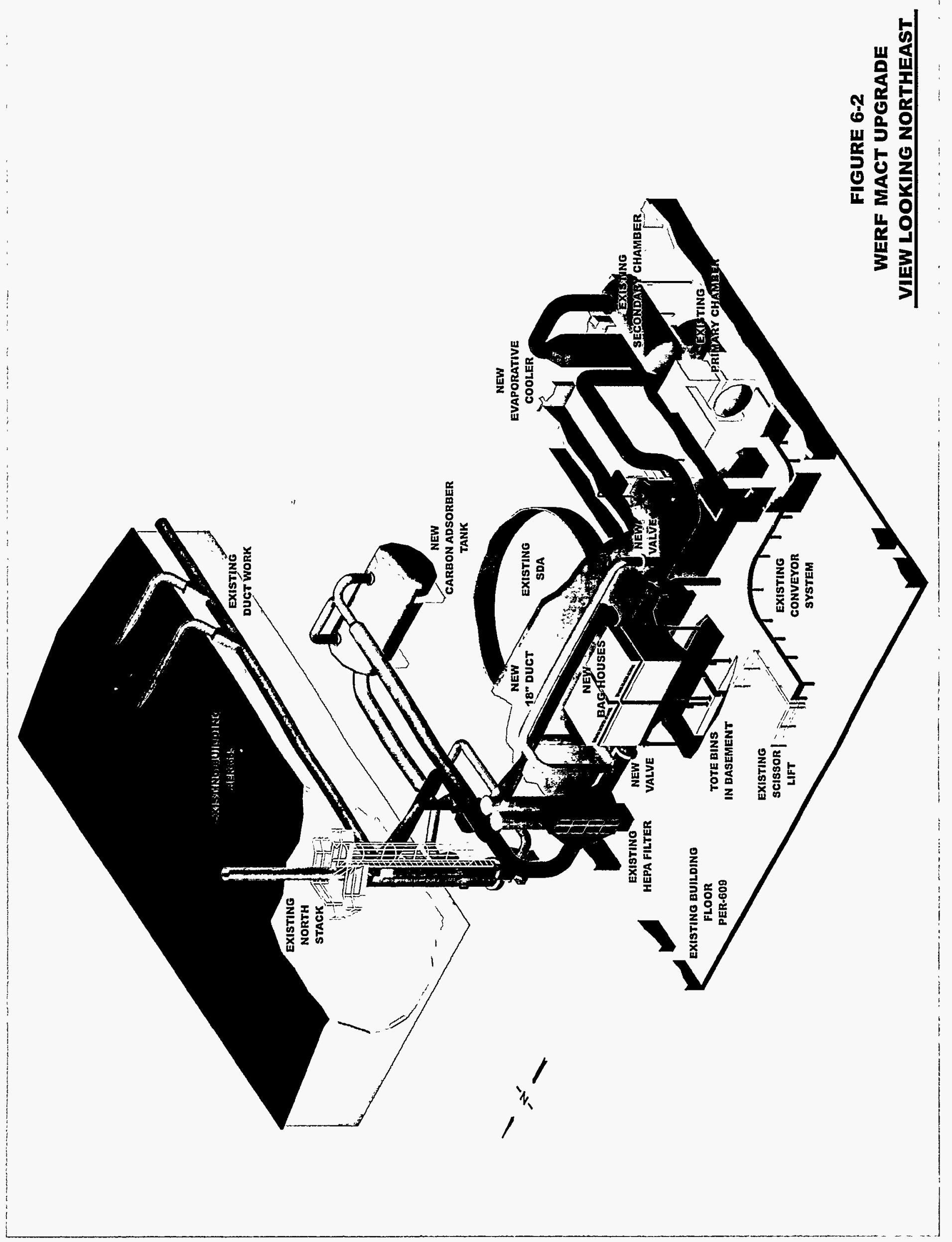




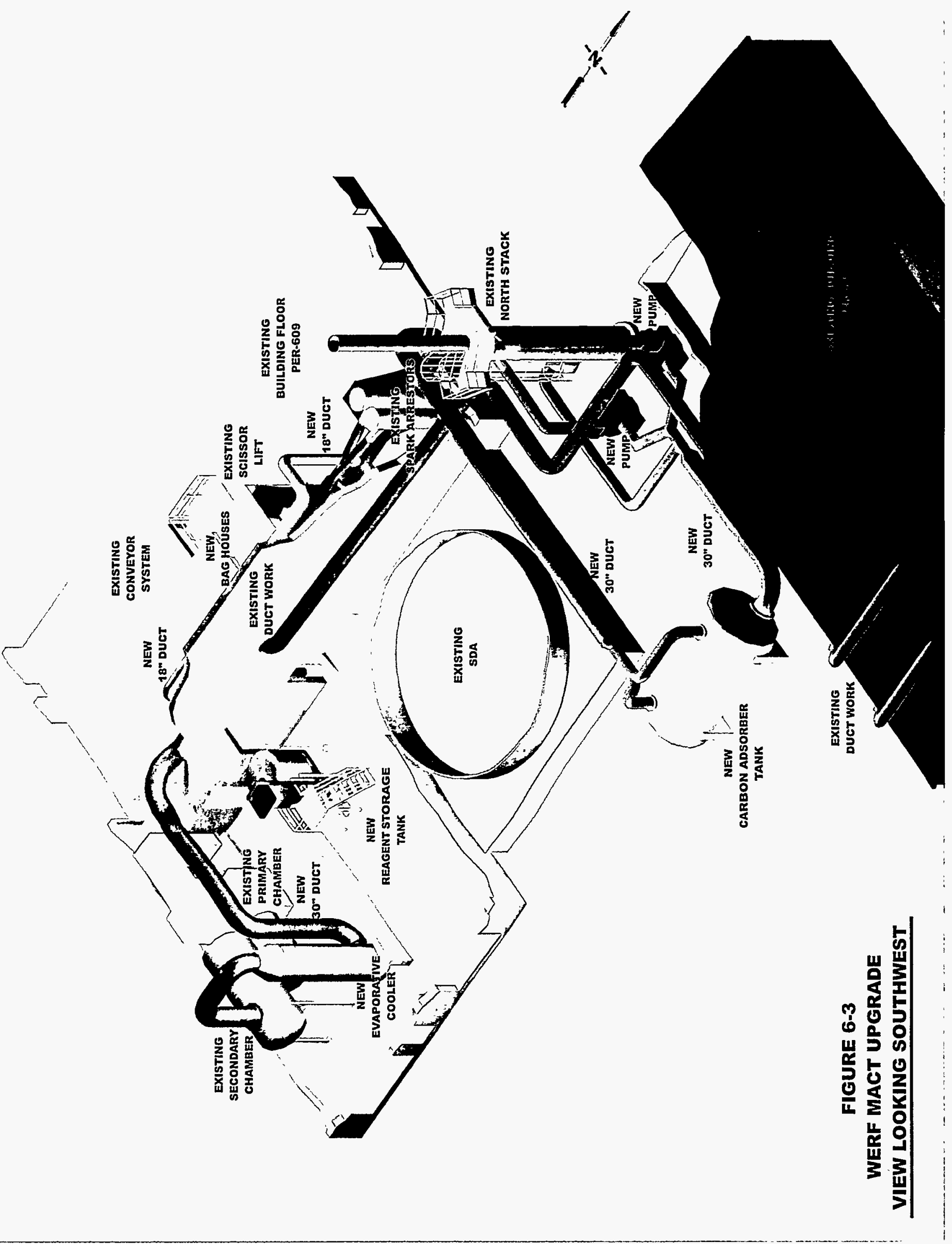




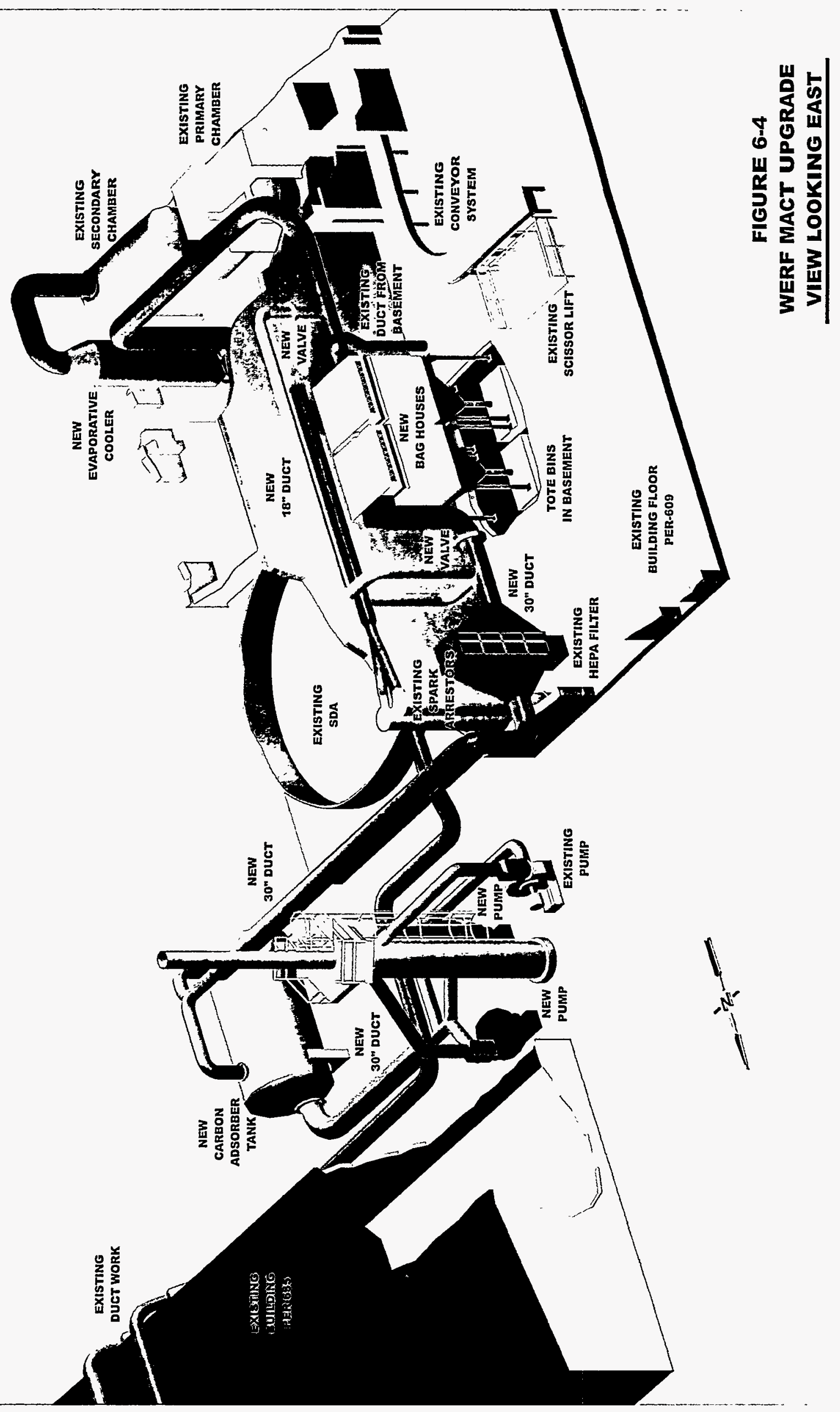


Table 6-1. Capital equipment costs.

Equipment Description

Evaporative cooler

Dry sorbent injection system

Fabric filter baghouse

Carbon adsorber

Induced draft fans

Total
(\$)

132,250

35,693

109,920

225,000

86,000

588,863

The engineering and design cost of $\$ 316,916$ was estimated from the type and number of drawings and specifications that are foreseen for the project. The quality assurance (inspection costs) were valued at $\$ 72,545$, which provides a total engineering, design, and inspection cost of $\$ 389,461$. Excluding contingency, the engineering, design, and inspection costs make up $13 \%$ of the total. This percentage is low when compared to most grass-root capital projects. However, since this project already has the necessary infrastructure and utilities in place, the amount of engineering and design in the estimate is considered reasonable.

The construction costs total $\$ 1,601,997$ without contingency added. This includes demolition of the existing equipment, ancillary equipment items and materials, and the capital equipment listed above. These costs do not include impact costs to WERF operations that relate to project construction and implementation. It is assumed that good engineering design and construction management practices will minimize these costs by scheduling incinerator shutdowns to coincide with equipment installation and tie-ins.

\subsection{Management Costs}

Project management costs are estimated to be $\$ 919,244$ or about $22 \%$ of the total estimated cost without contingency. Included in this cost are the environmental, safety, and health functions, RCRA permit modifications, and Permit to Construction and National Emission Standards for Hazardous Air Pollutants revisions. This cost also includes developing the operational procedures, conducting an operational readiness review, conducting a hazardous operations analysis, performing an independent design review, and performing system operation testing of the installed equipment.

\subsection{WERF Operational Cost Evaluation for MACT Upgrades}

WERF operates 24-hours per day, 365 days per year (less a ten day stand down over the Christmas holidays). Each work day is further broken down to three 8-hour shifts. A maximum of 1,095 shifts are possible per year $(3 * 365)$ for single shift process operations. Concurrent operations are possible for incineration/compaction, incineration/stabilization, incineration/macroencapsulation, stabilization/compaction, and stabilization/macroencapsulation.

\subsubsection{Incineration Costs Before Upgrades}

The operational goal is to actually be performing some form of waste treatment or volume reduction at least $84 \%$ of the time ( 900 shifts/1095 total shifts). Activities, that could preclude waste 
treatment, include: performance of equipment maintenance, personnel training, and personnel leave. These activities take away operational manpower required to perform various treatment processes. Table 6-2 provides a breakdown of the treatment and volume reduction processes.

Table 6-3 provides the operational costs by process for WERF MLLW treatment and LLW volume reduction. Individual fiscal year 1999 work packages were reviewed and costs allocated to each process as appropriate. Operational costs are ratioed from the percentage of shifts allocated to the process divided by the total number of shifts performed. Total cost of incineration is $\$ 8.5$ million per year.

\subsubsection{Incineration Costs After Upgrades}

The assumption is made that stabilization operations will be performed concurrently with incineration while the incinerator is operating. This will treat all of the fly ash generated from the new equipment. The remaining time when the incinerator is not operating will be dedicated to other process activities. Table 6-4 provides a breakdown of the treatment and volume reduction processes.

Table 6-5 provides the operational costs by process for WERF MLLW treatment and LLW volume reduction. Individual fiscal year 2001 work packages were reviewed and costs allocated to each process as appropriate. Operational costs are ratioed from the percentage of shifts allocated to the process divided by the total number of shifts performed. Total cost of incineration is $\$ 7.4$ million per year. Support costs use the same allocation as the before case. Maintenance of the new process equipment is assumed to the same as in the before case. Stabilization costs have significantly increased due to the increased volume of waste requiring stabilization. Additional process shifts have been allocated to perform this work, however, there is an inherent risk that this schedule is overly aggressive and may require additional resources. Until the new stabilization recipe is developed and the new stabilization process is put into use, these uncertainties will exist.

\subsection{Return on Investment}

Once the market has been established and the technical feasibility has been determined, the final question to be answered is, "What is the return on the funds invested?" A classical return on investment calculation is difficult to perform in the DOE system. The WERF incinerator is a non-profit operation, and there is no commercial alternative for treatment of this waste to benchmark against. A numerical return on investment cannot be assigned as the profitability standard in this case, but an overall judgement evaluation must be made against the alternatives. The alternatives are simple: one, upgrade WERF, or two, shut down WERF in 2001. The evaluation of these two alternatives will yield the real return on the investment of $\$ 4 \mathrm{M}$.

\subsubsection{Case One-Upgrading WERF}

The upgrade facility should have the capacity to incinerate $250 \mathrm{lbs} / \mathrm{hr}$, and therefore, would have the ability to treat the $4,652 \mathrm{~m}^{3}$ of mixed waste currently in the INEEL STP or in inventory on-site. In addition, the facility could treat the $5,348 \mathrm{~m}^{3}$ assumed to be generated in the years 2004-2010 for $10,000 \mathrm{~m}^{3}$ in ten years, at a rate equal to the generation rate. This capacity will require the investment of approximately $\$ 4 \mathrm{M}$ over the operating costs, amortized over ten years of waste treatment would be $\$ 400 / \mathrm{m}^{3}$ or $\$ 11 / \mathrm{ft}^{3}$. The incineration and stabilization costs before MACT upgrade totaled $\$ 9 \mathrm{M}$, while the post-upgrade costs total $\$ 9.6 \mathrm{M}$, but the volume of waste treated increases from $200 \mathrm{~m}^{3}$ to between $700-1300 \mathrm{~m}^{3}$ after the upgrade. This would reduce the unit cost of treatment at WERF by five times to approximately $\$ 272 / \mathrm{ft}^{3}\left(\$ 9.6 \mathrm{M} /\left(1,000 \mathrm{~m}^{3} \times 35.28 \mathrm{ft}^{3} / \mathrm{m}^{3}\right)\right.$ If we amortize the cost of the upgrade over ten years and $10,000 \mathrm{~m}^{3}$ of waste, the unit cost for treatment and disposal at WERF becomes $\$ 285 / \mathrm{ft}^{3}$. This is in the expected range of commercial services if they were available for this material. If the DOE complex 
Table 6-2. WERF Operating Schedule Before MACT

\begin{tabular}{lccc}
\hline \multicolumn{1}{c}{ Treatment Process } & Shift/yr & $\begin{array}{c}\text { Volume } \\
\left(\mathrm{m}^{3}\right) / \text { shift }\end{array}$ & $\begin{array}{c}\text { Volume } \\
\left(\mathrm{m}^{3}\right) / \mathrm{yr}\end{array}$ \\
\hline MLLW Incinerator & $420^{\mathrm{a}}$ & $0.17^{\mathrm{b}}$ & 71.4 \\
MLLW Stabilization & 30 & $0.5^{\mathrm{c}}$ & 15 \\
MLLW Ash Handling & $50^{\mathrm{d}}$ & $\mathrm{NA}$ & $\mathrm{NA}$ \\
MLLW Macroencapsulation & 20 & 0.21 & 4.2 \\
MLLW Sizing & 20 & 1.4 & 28 \\
LLW Compaction & 240 & 22.66 & 5,438 \\
LLW Sizing & 120 & 2.83 & 340 \\
Total & 900 & &
\end{tabular}

a. Based on WERF performing ten 14-day incineration campaigns per year.

b. $\quad 0.17 \mathrm{~m}^{3}$ per shift represents the historical portion of the original container volume processed over time.

c. Stabilization of one 71 -gal drum requires approximately 4 hours.

d. 5 shifts per campaign of ash handling are required to remove flyash and hearth ash from the incinerator system.

Table 6-3. Operational Costs before MACT Upgrades.

\begin{tabular}{|c|c|c|c|c|c|c|c|}
\hline WBS Number & Package Title & $\begin{array}{l}\text { Incineration } \\
(S)\end{array}$ & $\begin{array}{c}\text { Stabilization } \\
(\$)\end{array}$ & $\begin{array}{c}\text { Macro } \\
(\$)\end{array}$ & $\begin{array}{c}\text { MILW Sizing } \\
(\$)\end{array}$ & $\begin{array}{c}\text { Compaction } \\
(\$)\end{array}$ & $\begin{array}{c}\text { LLW Sizing } \\
(\$)\end{array}$ \\
\hline C. 1.02 .03 .01 .06 & LLW Volume Reduced - Ops & & & & & $1,529,015$ & 764,508 \\
\hline C. 1.02 .03 .01 .06 .01 & LLW WERF Operations & & & & & $1,338,959$ & 669,479 \\
\hline C.1.02.03.01.06.02 & $\begin{array}{l}\text { LLW Facility And Equipment } \\
\text { Maintained }\end{array}$ & & & & & 102,025 & 51,012 \\
\hline C.1.02.03.01.06.03 & $\begin{array}{l}\text { LLW Project Management and } \\
\text { Compliance }\end{array}$ & & & & & 88,031 & 44,018 \\
\hline C.1.02.03.02.07 & MLLW Treated-Ops & $8,478,174$ & 478,124 & 732,952 & 732,952 & & \\
\hline C.1 02.03 .02 .07 .01 & WERF Operations & $2,842,756$ & 181,453 & 120,968 & 120,968 & & \\
\hline C. 1.02 .03 .02 .07 .02 & MLLW Repackaging & $1,094,073$ & & & & & \\
\hline C.1.02.03.02.07.03 & $\begin{array}{l}\text { WERF Safety Analysis } \\
\text { Report/Permit Upgrade }\end{array}$ & 84,348 & 5,623 & 11,246 & 11,246 & & \\
\hline C.1.02.03.02.07.04 & RCRA Treatment Permitting & 570,326 & 38,022 & 76,043 & 76,043 & & \\
\hline C.1.02.03.02.07.05 & MLLW Engineering & 821,477 & 54,765 & 109,530 & 109,530 & & \\
\hline C. 1.02 .03 .02 .07 .06 & MLLW Maintenance & $1,379,816$ & 91,988 & 183,976 & 183,976 & & \\
\hline C.1.02.03.02.07.07 & MLLW Project Management & 256,467 & 17,098 & 34,196 & 34,196 & & \\
\hline C.1.02.03.02.07.08 & MLLW Treatment Compliance & $1,337,632$ & 89,175 & 178,351 & 178,351 & & \\
\hline C.1.02.03.02.07.09 & MLLW Treatment CE & 91,279 & & 18,641 & 18,641 & & \\
\hline C.1.02.03.02.01.05 & $\begin{array}{l}\text { Private Sector } \\
\text { Treatment/Disposal }\end{array}$ & & 28,980 & & & & \\
\hline
\end{tabular}

TOTAL

$8,478,174$

507,104

732,952

732,952

$1,529,015$

764,508 
Table 6-4. WERF Operating Schedule After MACT.

\begin{tabular}{|c|c|c|c|c|}
\hline & Treatment Process & Shifts/yr & $\begin{array}{c}\text { Volume } \\
\left(\mathrm{m}^{3}\right) / \text { shift }\end{array}$ & $\begin{array}{l}\text { Volume } \\
\left(\mathrm{m}^{3}\right) / \mathrm{yr}\end{array}$ \\
\hline \multicolumn{2}{|c|}{ MLLW Incineration } & $720^{2}$ & $1.7^{\mathrm{b}}$ & 71.4 \\
\hline \multicolumn{2}{|c|}{ MLLW Stabilization (ash) } & 460 & $0.5^{\mathrm{c}}$ & 230 \\
\hline \multicolumn{2}{|c|}{ MLLW Stabilization (non-ash) } & 85 & 0.5 & 42.5 \\
\hline \multicolumn{2}{|c|}{ MLLW Ash Handling } & $24^{d}$ & NA & NA \\
\hline \multicolumn{2}{|c|}{ MLLW Macroencapsulation } & 20 & 0.21 & 4.2 \\
\hline \multicolumn{2}{|c|}{ MLLW Sizing } & 20 & 1.4 & 28 \\
\hline \multicolumn{2}{|c|}{ LLW Compaction } & 74 & 22.66 & 1,677 \\
\hline \multicolumn{2}{|c|}{ LLW Sizing } & 47 & 2.83 & 133 \\
\hline \multicolumn{2}{|c|}{ Total } & 1,450 & & \\
\hline 2 & \multicolumn{4}{|c|}{ Based on WERF performing eight 30 -day incineration campaigns per year. } \\
\hline b. & \multicolumn{4}{|c|}{$1.7 \mathrm{~m}^{3} / \mathrm{shift}=250 \mathrm{lbs} / \mathrm{hr} * 8 \mathrm{hrs} / \mathrm{shift} /\left(550 \mathrm{Kg} / \mathrm{m}^{3} * 2.2 \mathrm{lbs} / \mathrm{kg}\right)$} \\
\hline c. & \multicolumn{4}{|c|}{$\begin{array}{l}\text { The dry absortent added to remove HCl from the offgas will increase to quantity to flyash generated by approximately } 15 \text { times. Stabilization of one } 71 \text {-gal } \\
\text { drum requires approximately } 4 \text { hours. }\end{array}$} \\
\hline d. & \multicolumn{4}{|c|}{$\begin{array}{l}3 \text { shifis per campaign of ash handling are required to remove flyash and hearth ash from the incinerator system. Decrease is due to the simplier flyash } \\
\text { removal system. }\end{array}$} \\
\hline
\end{tabular}

Table 6-5. Operational costs after MACT upgrades.

\begin{tabular}{|c|c|c|c|c|c|c|c|}
\hline WBS Number & Package Title & $\begin{array}{l}\text { Incineration } \\
(\$)\end{array}$ & $\begin{array}{c}\text { Stabilization } \\
(\$)\end{array}$ & $\begin{array}{c}\text { Macro } \\
(\$)\end{array}$ & $\begin{array}{c}\text { MLLW Sizing } \\
(\$)\end{array}$ & $\begin{array}{l}\text { Compaction } \\
(\$)\end{array}$ & $\begin{array}{l}\text { LLW Sizing } \\
(\$)\end{array}$ \\
\hline C.1.02.03.01.06 & LLW Volume Reduced - Ops & & & & & 440,383 & 254,148 \\
\hline C. 1.02 .03 .01 .06 .01 & LLW WERF Operations & & & & & 251,276 & 159,594 \\
\hline C. 1.02 .03 .01 .06 .02 & $\begin{array}{l}\text { LLW Facility And Equipment } \\
\text { Maintained }\end{array}$ & & & & & 101,515 & 50,758 \\
\hline C. 1.02 .03 .01 .06 .03 & $\begin{array}{l}\text { LLW Project Management and } \\
\text { Compliance }\end{array}$ & & & & & 87,591 & 43,796 \\
\hline C.1.02.03.02.07 & MLLW Treated-Ops & $7,443,329$ & $2,153,325$ & 578,222 & 578,222 & & \\
\hline C. 1.02 .03 .02 .07 .01 & WERF Operations & $2,526,343$ & $1,898,170$ & 67,912 & 67,912 & & \\
\hline C. 1.02 .03 .02 .07 .02 & MLLW Repackaging & $1,089,661$ & & & & & \\
\hline C. 1.02 .03 .02 .07 .03 & $\begin{array}{l}\text { WERF Safety Analysis } \\
\text { Repor/Permit Update }\end{array}$ & 83,927 & 5,595 & 11,190 & 11,190 & & \\
\hline C.1.02.03.02.07.04 & RCRA Treatment Permitting & 93,431 & 6,229 & 12,457 & 12,457 & & \\
\hline C.1.02.03.02.07.05 & MLLW Engineering & 720,662 & 48,044 & 96,088 & 96,088 & & \\
\hline C.1.02.03.02.07.06 & MLLW Maintenance & $1,368,101$ & 91,207 & 182,413 & 182,413 & & \\
\hline C.1.02.03.02.07.07 & MLLW Project Management & 216,014 & 14,401 & 28,802 & 28,802 & & \\
\hline C.1.02.03.02.07.08 & $\begin{array}{l}\text { MLLW Treatment } \\
\text { Compliance }\end{array}$ & $1,345,193$ & 89,680 & 179,359 & 179,359 & & \\
\hline C.1.02.03.02.07.09 & MLLW Treatment CE & & & & & & \\
\hline C.1.02.03.02.01.05 & $\begin{array}{l}\text { Private Sector } \\
\text { Treatment/Disposal }\end{array}$ & & 57,960 & & & & \\
\hline & TOTAL & $7,443,329$ & $2,211,285$ & 578,222 & 578,222 & 440,38315 & 254,148 \\
\hline
\end{tabular}


does ever move to a charge back scenario, a unit price of $\$ 300 / \mathrm{ft}^{3}$ is very competitive, and the facility would evolve from a cost center into a profit center. It is important to note that the capacity of $1,000 \mathrm{~m}^{3} / \mathrm{yr}$ depends on the ability to co-mingle small waste streams and optimize them into large campaigns, which depends on identifying a reliable DOE or commercial disposal facility.

\subsubsection{Case Two-Shutdown WERF}

Failure to comply with the MACT rule would force the facility to shutdown by April 2001. At its current capacity, WERF could incinerate $500 \mathrm{~m}^{3}$ before shutdown. Once the decision not to upgrade is made, further off-site shipment would be suspended, and the facility would concentrate on on-site waste. Unless TSCA or CIF and their respective states open up the borders to off-site waste, the DOE complex could have an additional $9500 \mathrm{~m}^{3}$ of mixed waste still in storage waiting for treatment and disposal in 2010.

In 1992, the Savannah River Site estimated the fixed and variable costs associated with managing mixed waste. The fixed costs were $1,000 / \mathrm{ft}^{3}$, while the variable costs totaled $\$ 1,100 / \mathrm{ft}^{3}$. If we assume the fixed costs are purely storage and the variable costs cover treatment and disposal, then by 2010 , the complex would have spent an additional $\$ 335 \mathrm{M}$ for management but not moved this material to ultimate disposal. No commercial alternative, with the exception of the Advanced Mixed Waste Treatment Project, is on the horizon to consider for treatment of this material. The Advance Mixed Waste Treatment Project alternative was briefed to the DIS conference in August 1998, and there was significant discussion on sending mixed waste for treatment and receiving a pro-rated share of transuranic waste in return.

\subsubsection{Conclusions}

The cost to upgrade WERF is small $\left(\$ 11 / \mathrm{ft}^{3}\right)$ compared to the capacity increase, which would result. The facility is sound from an engineering, political, and regulatory perspective and has a nearly indefinite service life with routine maintenance. The upgraded facility will be able to treat most of the incinerable mixed waste generated in the western United States for the near future. Investing in the WERF facility keeps the INEEL moving waste toward ultimate disposal, as opposed to shutting down a permitted and operating facility while hoping for two state governors to have a change of heart, or for a transuranic waste treatment facility, yet to be constructed, to have the excess capacity to transform MLLW to transuranic waste to be easier to dispose. The return on the investment of $\$ 4 \mathrm{M}$ is a capable facility to continue moving mixed waste to disposal and working off the DOE backlog. 


\section{PROJECT SCHEDULE}

Several important regulatory events will dictate the schedule to upgrade WERF for MACT compliance within the three-years allotted by the EPA. These include requirements set forth in the June 19, 1998, final rule for a notice of intent to comply (NIC), public meeting, and progress report. Additionally, modifications to air and RCRA permits will be required and NEPA issues addressed.

The MACT final rule is expected to be published in April 1999. If the WERF incinerator is to continue operation, the facility must be MACT compliant within three years of the date the final ruling is issued. This three-year period includes the time necessary to complete regulatory requirements, design, construction, and testing of MACT compliant equipment, and performance testing and certification of compliance.

\subsection{Regulatory Considerations}

\subsubsection{Notification of Intent to Comply}

A NIC must be submitted to the State of Idaho within one year of the publishing of the MACT final standards in the Federal Register. The NIC documents the source's intentions towards coming into compliance. For facilities intending to comply, the NTC will include a schedule regarding the beginning and completion of engineering studies; the dates by which contracts will be awarded or purchase orders will be issued; the date construction applications will be submitted; the dates by which on-site construction, installation, or process change is to be initiated and completed; and the date by which final compliance is to be achieved.

For any source that does not intend to comply with MACT standards, the NIC must certify that the source will stop burning hazardous waste on or before two years after the effective date of the MACT standards. The NIC must include a schedule of key dates for steps to be taken to meet this goal, including the date of submittal of RCRA closure documents. An exception is a source that is necessary to combust hazardous waste from another on-site source while that other source is coming into compliance with the MACT standards. The source not intending to comply, in this situation, may burn hazardous waste during the third year up to the MACT compliance date.

\subsubsection{Notification of Intent to Comply Public Meeting and Notice}

Prior to submittal of the final NIC, the source must conduct an informal public meeting to discuss anticipated plans for achieving compliance with the MACT standards. This meeting must take place no later than 10 months after the standards are promulgated. A notice that includes pertinent information announcing the public meeting must be made, at least 30 days prior to the date of the meeting; the notice must describe how a copy of the draft NIC can be obtained and who can be contacted to answer questions.

\subsubsection{RCRA Class 1 Modification Request}

Facility changes to meet MACT standards will require a modification to the facility RCRA permit. EPA has streamlined the modification process to allow this request to be classified as a Class 1 modification, as long as prior permitting agency approval is obtained. If adopted by the State of Idaho, the permitting agency would have 90 days (with a possible one-time extension for up to 30 days) from the time the request is received to approve or deny the Class 1 permit modification request. If a determination is not made by the permitting agency within the 90-day time-frame, the request would be considered approved. If the permitting agency denies the request (e.g., if the request contained insufficient information), a resubmittal would be made, and a new 90-day review period would be 
initiated. Once approval is obtained from the permitting agency, in accordance with the current Class 1 modification requirements, the permittee must inform the public about the modifications within 90 days.

\subsubsection{Progress Report}

No later than two years after the effective date of the MACT standards, a progress report is due to the permitting agency that tracks the source's actions toward compliance. At this time, the source should have a completed engineering design for the physical modifications necessary to meet the MACT emissions standards; construction applications should have been submitted to the applicable regulatory authorities, and a binding contractual commitment to purchase, fabricate, and install equipment and structures shall have been made.

The progress report will contain information demonstrating that these obligations have been met and that the facility has made adequate progress towards compliance. The report must also contain a schedule listing key dates for activities that will bring the source into compliance. Key dates include construction contract and equipment supply contractors bid and award dates, ground breaking, completion of specifications and drawings, equipment deliveries, intermediate construction completions, testing, anticipated dates of any permit or license approvals, and the projected date of facility compliance. The progress report is a revision to the information and schedule that will have been submitted in the NIC.

Sources that do not intend to comply with the MACT standards will submit in their progress report on the date that the source stopped burning hazardous waste and the date that RCRA (Hazardous Waste Management Act) closure documents were submitted.

\subsubsection{Permit to Construct/National Emission Standards for Hazardous Air Pollutants}

A revision to the Permit to Construct and the National Emission Standards for Hazardous Air Pollutants (NESHAP)s report required for the WERF level of radioactive emissions (the WERF Emissions Sources NESHAPS Monitoring Calculations Report submitted to EPA Region X) will be required prior to the start of construction, because the new construction is considered a major modification to an existing facility. These modifications include air dispersion modeling and calculations of both hazardous and radioactive emissions. EPA may issue the Permit to Construct changes to these requirements that, in view of the RCRA permit modification requirements, may only be required to be submitted during the next (5-year) regularly scheduled update of the air permit. The integration of air and RCRA permitting is expected to be more fully discussed in the final rule.

\subsubsection{National Environmental Policy Act of $\mathbf{1 9 6 9}$}

At a minimum, a completed Environmental Checklist will be necessary to assess National Environmental Policy Act (NEPA) issues. The WERF MACT upgrade likely will qualify for a categorical exclusion per appendix B to Subpart D of the DOE NEPA regulations (10 CFR 1021). ${ }^{57}$

If the facility upgrade does not qualify for a categorical exclusion, DOE will probably prepare a supplement analysis to determine if the existing site-wide Environmental Impact Statement adequately bounds WERF MACT upgrade issues. A supplement analysis will conclude one of three things: (1) The existing site Environmental Impact Statement must be supplemented with an analysis of the environmental impacts expected from WERF MACT upgrades; (2) a new Environmental Impact Statement must be prepared addressing environmental impacts expected from WERF MACT upgrades; or (3) no further NEPA analysis is required (i.e., the existing Environmental Impact Statement adequately bounds the environmental issues raised by WERF MACT upgrades). 


\subsection{General Plant Project Construction Project}

A General Plant Project construction project is estimated to cost less than 5 million dollars. Based on the planning cost estimate discussed in section 6 of this report, it is assumed that an upgrade to WERF to comply with MACT requirements would be categorized as a General Plant Project construction project.

A General Plant Project construction project typically consists of a project definition phase, conceptual design phase, title (detail) design phase, construction phase, and inspection/transfer. It is anticipated that 21 months will be required to complete this General Plant Project construction project. The following sections discuss in more detail the phases of this General Plant Project construction project.

\subsubsection{Project Definition Phase}

In this phase of the project, the functional and operational requirements associated with the ensuing project are identified. The functional and operational requirements ensures that the basic requirements of the project are identified. Project design requirements are then developed from the functional and operational requirements and serve as a basis for the conceptual design. It is anticipated that this phase of the WERF MACT upgrade project will require approximately 2 months.

\subsubsection{Conceptual Design Phase}

The conceptual design encompasses those efforts to do the following:

- Develop a project scope that will satisfy facility needs

- Ensure project feasibility and attainable performance levels

- Develop reliable cost estimates and realistic schedules

- Develop project criteria and design parameters for all engineering disciplines, including the identification of applicable codes and standards, quality assurance requirements, environmental studies, materials of construction, and any other features or requirements necessary to describe the project.

Approximately 4 months have been anticipated for completion of this phase of the project.

\subsubsection{Title (Detail) Design}

This phase continues the design effort utilizing the conceptual design and the project design criteria as a basis for project development. Long-lead procurement items are identified. The preparation of final working drawings, specifications, bidding documents, and cost estimates are completed. A firm construction and procurement schedule is established. Approximately 6 months have been anticipated for completion of this phase of the project.

\subsubsection{Construction}

Construction is the combination of engineering, procurement, installation, assembly, and fabrication activities required to modify a facility. Request for Proposal packages are prepared and issued to prospective construction subcontractors. A construction subcontractor is selected and the work completed. Approximately 7 months have been anticipated for completion of this phase of the project. 


\subsubsection{Inspection/Project Transfer}

Inspection and testing of the installed systems are performed during this phase of the project. Nonconformances and discrepancies are identified and documented. The installed systems are transferred to the program (user) when all discrepancies are dispositioned and the system is in working order. Two months have been anticipated for this phase of the project.

\subsection{Recommended Schedule}

The regulatory schedule mandated by the MACT rules will drive the construction of WERF upgrades. Figure 7-1 shows the MACT compliance dates and the recommended environmental/construction schedule based on those dates. Once the MACT regulations are final (currently April 1999), pre-conceptual design should be initiated. Simultaneously, preparation of the RCRA permit modification request should also begin. Information gathered for these two efforts will be important when writing the NIC; this task should begin shortly after MACT promulgation.

When balancing the regulatory schedule with the construction schedule, two schedule ties are important in the early phase. One tie is the relationship between the design of the system upgrades to the completion of a detailed RCRA modification request. The RCRA modification request will require fairly detailed design information on the new equipment and operation changes that are a result of the new standards. Secondly, it is prudent that the bid and procurement activity not begin until approval is granted for the RCRA permit modification request. This ties RCRA permit modification approval ( 90 days from the time of submittal, unless a 30-day extension option is exercised or the request is deemed incomplete) to procurement start.

Considering these two ties, it is recommended that the RCRA Class 1 modification request be submitted as soon as sufficient design details are known. When merging this date with the desired construction schedule, this date should be no later than 9 months after MACT promulgation.

The final rule requires facility owners or operators to complete the NIC in order to benefit from the streamlined modification procedures. Subsequently, the final NIC, although not due until one year after the MACT promulgation, must be issued earlier (at 9 months) in order to allow timely submittal of the RCRA modification request. In turn, the public meeting should occur at least 1 month before the NIC is finalized, making the draft NIC due no later than about 7 months after MACT promulgation.

This recommended schedule allows construction activities to be completed on or before the end of Year 2. The information required for the progress report, due at the end of Year 2, as well, will be easily summarized at this time.

Allowing 8 to 9 months for construction, this schedule leaves at least 1 year for the completion of the performance test and report and establishing WERF compliance with the MACT emission standards by the end of the Year 3. During this time preliminary tests, such as mini-burns, can be performed to ensure the success of the performance test (trial burn). 


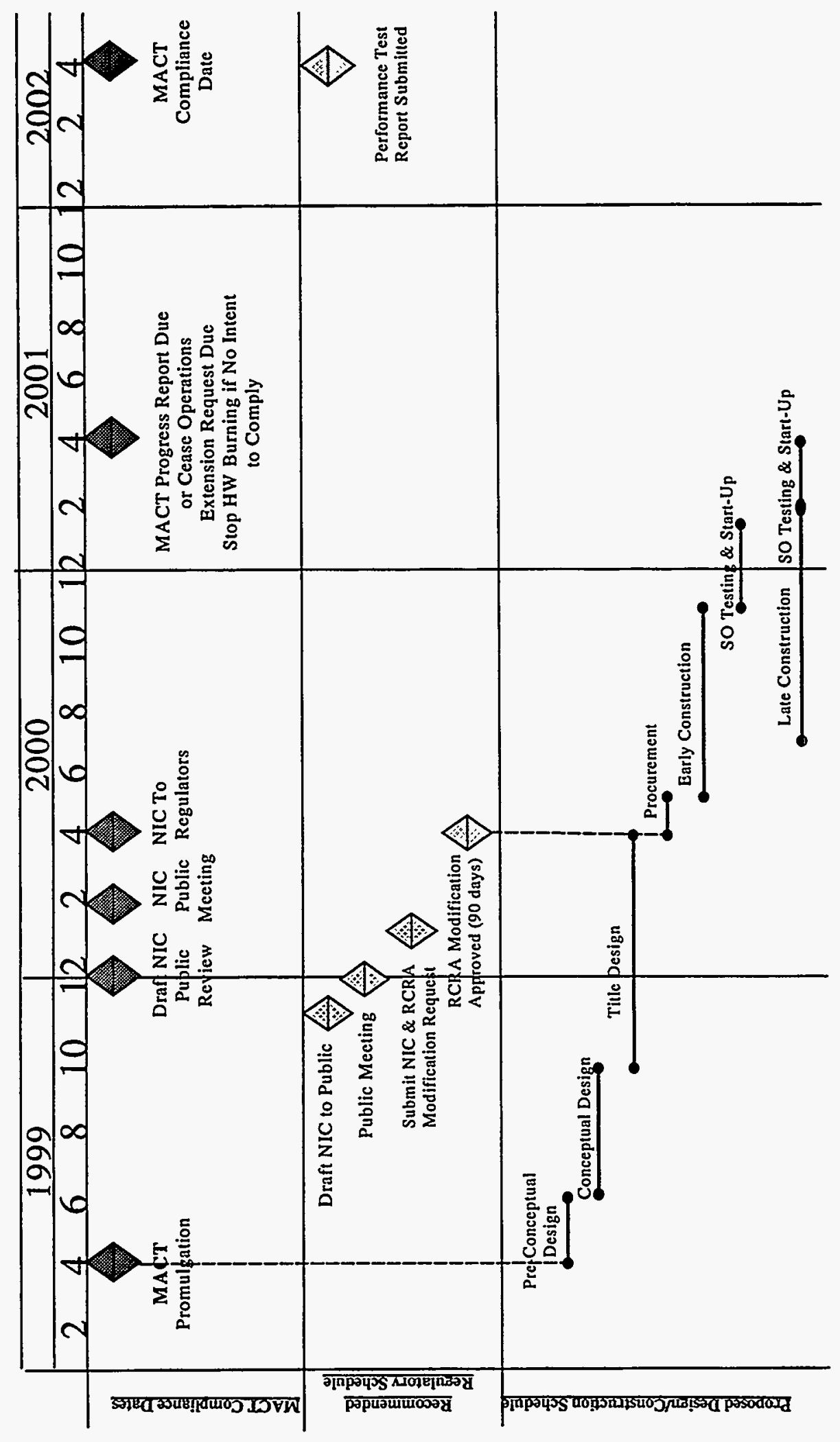

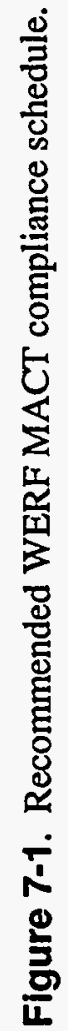




\section{SUMMARY}

As discussed in Sections 4 and 5 of this report, the WERF Feasibility Study Project Team developed four practicable offgas treatment processes, which, if installed, would enable the WERF to meet the anticipated MACT emission limits for $\mathrm{HCl}, \mathrm{Hg}$, and $\mathrm{D} / \mathrm{F}$. The selected option is the partial quench with DSI for $\mathrm{HCl}$ removal followed by carbon adsorption for $\mathrm{Hg}$ control. This option can be implemented within the $\$ 5 \mathrm{M}$ General Plant Project funding limit.

\subsection{Technical Risks and Risk Mitigation}

The following is a discussion of the perceived technical risks associated with the project along with the information used to mitigate the risks.

\subsubsection{Adequacy of $\mathrm{HCl}$ Control}

Dry sorbent injection is an $\mathrm{HCl}$ control technology commonly used for medical waste incinerators similar in design to the WERF incinerator. When feeding $20 \mathrm{lbs} / \mathrm{hr}$ of chlorine to the WERF incinerator, the assumed design parameters of $97.2 \% \mathrm{HCl}$ removal can be readily supported by trial burn information from existing facilities when hydrated lime is used as the neutralizing reagent with a stoichometric ratio of 2 to1. (Note: Sodium bicarbonate or sodium carbonate can also be used to neutralize HCl.) INEEL personnel visited two commercial medical waste incinerators equipped with DSI systems similar to the chosen option. The visitations confirmed that the required equipment is commercially available and relatively simple to operate.

\subsubsection{Adequacy of $\mathrm{Hg}$ Control}

A fixed bed of sulfur-impregnated carbon is the technology of choice for $\mathrm{Hg}$ control, and information provided by Nucon was used to size the carbon adsorption bed. Although confirmation of the vendor data was not possible, using excessive quantities of activated carbon for $\mathrm{Hg}$ control is well known and documented. The advantage for using sulfur-impregnated activated carbon is that much smaller quantities are required. The carbon bed was conservatively sized for a total $\mathrm{Hg}$ capacity in excess of a 10-year life span when feeding $10 \mathrm{~g} / \mathrm{hr}$ of the volatile metal.

\subsubsection{Adequacy of Dioxin/Furan Control}

There are two features of the proposed APCS that will enable the WERF facility to meet the MACT standard for D/F emission. The first is the use of a partial quench to quickly cool the offgas. Cooling the offgas from 1900 or $2000^{\circ} \mathrm{F}$ to $350^{\circ} \mathrm{F}$ in 0.5 to 1 second will minimize the formation conditions (long gas residence times in the 400 to $900^{\circ} \mathrm{F}$ range) for $\mathrm{D} / \mathrm{F}$. The second method for $\mathrm{D} / \mathrm{F}$ control involves the carbon bed that is designed for the Hg capture. Should D/F form, the activated carbon will adsorb most of them in addition to the $\mathrm{Hg}$. As stated above, the carbon bed is conservatively sized such that co-adsorption of D/F will not adversely affect the Hg capture efficiency.

\subsubsection{Safety}

A preliminary process hazards analysis was performed for the recommended option. Although the analysis was based on limited process design information, no "show-stoppers" were identified. The critique did reveal that redundant process control is required for the gas quench system to avoid damaging downstream equipment. A summary of the analysis is located in Appendix C. 


\subsubsection{Facility Assessment}

If implemented, the DSI system will be retrofitted into the existing process and facility. As discussed in Section 3, a review of the WERF facility was conducted to ensure that there are no other site or mechanical conditions that would require significant equipment repair or replacement that would adversely affect the project. The facility analysis revealed no significant structural or mechanical problems that would oppose the decision to implement the chosen alternative for complying with the MACT rule.

\subsubsection{Cost Estimate}

The planning cost estimate for implementing the DSI system is $\$ 4.2 \mathrm{M}$ which includes the design, procurement and installation of a new offgas cooler, a DSI system with reagent storage, a fabric filter baghouse, a carbon adsorber, and two offgas induced draft exhaust fans. Except for the induced draft fans, vendor quotations were obtained for these equipment items. Preliminary equipment layout drawings and material take-offs were also used to estimate the cost for installing the recommended APCS.

The cost estimate includes a $24 \%$ contingency. This contingency is within the accuracy of the cost estimate which is considered to be -25 to $+30 \%$ of the total cost without contingency. 


\section{REFERENCES}

1. Idaho National Engineering and Environmental Waste Experimental Reduction Facility (WERF) Incinerator Trial Burn-May and July, Vol. II, Lockheed Martin, INEEL/EXT-9801180, October 1998.

2. Federal Register, 61 FR 17358 "Hazardous Waste Combustors; Revised Standards: Proposed Rule," U.S. Environmental Protection Agency, April 19, 1996.

3. Federal Register, 62 FR 24212, "Revised Technical Standard for Hazardous Waste Combustion Facilities; Proposed Rule," U.S. Environmental Protection Agency, May 2, 1997.

4. Federal Register, 63 FR 33782, "Hazardous Waste Combustors, Revised Standards; Final Rule - Part 1," U.S. Environmental Protection Agency, June 19, 1998.

5. Accelerating Cleanup: Paths to Closure, DOE/EM-0362, U.S. Department of Energy, Office of Environmental Management, June 1998.

6. 1995 Mixed Waste Inventory Report, M96-GT-029, U.S. Department of Energy, Office of Environmental Management, 1996.

7. N-Soelberg, et al., MACT System Integration Study Draft Status Report, Lockheed Martin Idaho Technologies Company, October 23, 1998.

8. Idaho National Engineering Laboratory Reusable Property, Recyclable Materials, and Waste Acceptance Criteria - RRWAC, Rev. 5, DOE/ID-10381, INEL, Idaho Falls, ID, October 1995.

9. Senior, C. L., et. al., "A Fundamental Study of Mercury Partitioning in Coal Fired Power Plant Flue Gas," 97-WP72B.08. Presented at the Air and Waste Management Association's 90th Annual Meeting and Exhibition, Toronto, Ontario, Canada, June 8-13, 1997.

10. Code of Federal Regulations, 40 CFR 266, "Standards for Materials being Recycled Reused," July 1, 1998.

11. D. K. Morton, Fatigue Analysis of Incinerator Lower Ignition and Upper Combustion Chambers, WERF-EDF-0121, April 1992.

12. C. K. Branter, WERF Incinerator Inspection, WERF-EDF-0134, May 1992.

13. R. K. Blandford, Thermal Effects Evaluation of the WERF Incinerator Reinforced Concrete Floor, WERF-ED-0122, April 1992.

14. D. A. Conley, Analysis of Corrosion Coupons Removed from Incinerator Off gas System, WERF-EDF-0153, August 1992.

15. B. C. Satterthwaite, Crack Evaluation of the Main Off gas High Efficient Particulate Air (HEPA) Filter Housing in the Waste Experimental Reduction Facility (WERF) Highbay, WERF-EDF-0185, November 1992. 
16. D. A. Conley, WERF North Stack (PER-755) Corrosion Sample Analysis, WERF-EDF0128 , April 1992.

17. Soelberg, N., K. Liekhus, and A. Chambers, 1997a, "APCS Conceptual Design and Evaluation Study” INEEL/EXT-97-01138, Draft, September.

18. K. C. Galbreath and C. J. Zygarlicke, 1996, Mecury speciation in coal combustion and gasification flue gases. Environ. Sci. Technol., 30(8), 2421-2425.

19. Soelberg, N., "Mercury Emissions Control Study for Mixed Waste Thermal Treatment," presented at the National Technical Workgroup 1997 Meeting, Newport, CA, November 5-7, 1997.

20. Gaspar, J. A., N. C. WIDMER, J. A. COLE, and W. R. SEEKER, 1997, "Study of Mercury Speciation in a Simulated Municipal Waste Incinerator Flue Gas," 1997 International Conference on Incineration and Thermal Treatment Technologies, Oakland, California, May 12-16, 1997.

21. Young, B., "Mercury Removal from Off gas Systems," white paper submitted to the DOE Mixed Waste Focus Area, September 4, 1997.

22. K. C. Galbreath and C. J. Zygarlicke, "Mercury speciation in coal combustion and gasification flue gases," Environ. Sci. Technol., 30(8), 2421-2425 (1996).

23. J. A. Gaspar, 1998, New processes for the removal of mercury from incinerator flue gases. International Conference on Incineration and Thermal Treatment Technologies, Salt Lake City, UT, 565-568.

24. Lausman, R. and L. Lavely, "Controlling Air Toxics Emissions Poses Challenges," Power Engineering, August 1997.

25. CRC Handbook of Chemistry and Physics, $59^{\text {th }}$ ed., CRC Press, 1978.

26. B. Hall, P Schager and O. Lindqvist, "Chemical reactions of mercury in combustion flue gases," Water, Air, and Soil Pollution, 56, 3-14 (1991).

27. Fellows, R.L., "Use of Gold (AU) and Platinum (Pt) Metals for Removal of Mecury (Hg) from Air: A Review and Evaluation of the Technologies," K/TSO-59, Lockheed Martin Energy Systems, Inc, East Tennessee Technology Park, Oak Ridge, TN, February 1998.

28. L. Liberti et al., 1998, Mercury removal with powdered activated carbon from flue gases at the Coriano municipal solid waste incineration plant. Waste Management Res., 16(2), 183-189.

29. R. D. Vidic and J. B. McLaughlin, 1996, Uptake of elemental mercury vapors by activated carbon. J. Air Waste Management Assoc., 46, 241-250.

30. EPA, "Draft Technical Support Document for NWC MACT Standards (NODA), Volume I: MACT Evaluations Based on Revised Database," April 1997. 
31. Krause, C., "ORNL quantifies important highly water-soluble mercury gas in air," ORNL Press Release found on URL Release/archive/mr960122-00.html, Jan. 22, 1995.

32. Vidic, R. D., W. Liu, and T. D. Brown, "Adsorption Efficiency of Activated Carbon-Based Sorbents for Elemental Mercury Vapors: Impact of Sulfur Impregnation Method," Submitted for publication, October 16, 1994.

33. Chang, R. and D. Ownes, "Developing Mercury Removal Methods for Power Plants," EPRI Journal, July/August 1994.

34. Siret, B. and S. Eagleson, "A New Wet Scrubbing Technology for Control of Elemental (Metallic) and Ionic Mercury Emissions," 1997 International Conference on Incineration and Thermal Treatment Technologies, Oakland, California, May 12-16, 1997.

35. Livengood, D. C. and M. H. Mendelsohn, "Enhanced Control of Mercury Emissions Through Modified Specification," 97-WP72B.01, Air and Waste Management Association 90 ${ }^{\text {th }}$ Annual Meeting and Exhibition, Toronto, Ontario, Canada, June 8-13, 1997.

36. Meij, R., "The Fate of Mercury in Coal-Fired Power Plants and the Influence of Wet Flue-Gas Desulphurization," Water, Air, and Soil Pollution, 56:21-33, 1991.

37. EPA, "Mercury Study Report to Congress," EPA-452/R-97-003, December 1997.

38. Connet, E. and P. Connet, "A Study of Mercury Contamination in the U.S.A.," A publication of Work On Waste USA, Inc., URL http://ecologia.nier.org/english/level1/wastenot/wn207.htm, September 1992.

39. S. V. Krishnan, B. K. Gullet, and W. Jozewicz, 1994, Sorption of elemental mercury by activated carbons. Environ. Sci. Technol., 28 1506-1512.

40. Nucon International, Inc., "Design and Performance Characteristics of MERSORB® Mercury Adsorbents in Liquids and Gases," NUCON 11B28, August 1995.

41. Livengood, D. C., H. S. Huang, and C. B. Sedman, "Mercury Sorption Mechanisms and Control by Calcium-Based Sorbents," 96-WP64B.05, Air and Waste Management Association $89^{\text {th }}$ Annual Meeting and Exhibition, Nashville, TN, June 23-28, 1996.

42. Roberts, D. L., "Proof-of-Concept Field Testing of a Novel Regenerable Process for Mercury Removal and Recovery from Flue Gases," R440095F01, Final report for Fiscal Year 1995 prepared for Sandia National Laboratories by ADA Technologies, Inc., September 25, 1995.

43. Roberts, D. L., T. E. Broderick, and R. M. Stewart, "Removing and Recovering Mercury from Off gases of Thermal Treatment Processes," paper number 98-WP79A.03 submitted for presentation at the Air \& Waste Management Association $91^{\text {st }}$ Annual Meeting \& Exhibition, San Diego, CA, June 1419, 1998

44. P. Acharya, S. G. DeCicco, and R. G. Novak, 1991, Factors that can influence and control the emissions of dioxins and furans from hazardous waste incinerators. J. AWMA, 41, 1605-1615. 
45. C. R. Ullich, J. Blandamer, and P. L. Pojawis, 1996, Carbon injection experience with dioxin and mercury removal in a hazardous waste incinerator gas cleaning train. Proc. Of $89^{\text {th }}$ Annual AWMA Meeting, Nashville, TN.

46. A. Sigg, 1994, Control of dioxin and furan emissions from waste incineration systems. Proc. of incineration Conf., Houston, TX.

47. J. Mehta et al., 1997, Measurement and control of dioxins and furans from a hazardous waste incinerator. Proc. of $90^{\text {th }}$ Annual AWMA Meeting, Toronto, Canada.

48. Technical Support Document for HWC MACT Standards - Volume IV: Compliance with the proposed MACT standards (Draft), February 1996, U. S. Environmental Protection Agency, EPA 600/7-79-065.

49. Code of Federal Regulations, 40 CFR 60, Appendix A, July 1, 1996.

50. Gnyp A.W. et al., 1979, Long-term field evaluation of continuous particulate monitors. Proceedings: Advances in Particle Sampling and Measurement.

51. Wedding J.A. and M.A. Weigand, 1993, An automatic particle sampler with beta gauging. J. Air Waste Manage. Assoc., 43, 475-479.

52. J. S. Vavruska, Equinox Ltd., Santa Fe, NM, Private Communication, October 7, 1998.

53. R. P. Bundy, 1997, Maximizing the operating efficiency of a dry scrubber on medical waste incineration. AWMS Annual Meeting, Toronto.

54. "HCI Removal with Sodium Bicarbonate Injection at Colorado Incineration Services, Inc., Denver, CO.”, Bicarb-Bulletin, Church \& Dwight Co., Inc., Princeton, NJ.

55. "HCl Removal with Sodium Bicarbonate Injection at Swedish Medical Center, Seattle, WA, Bicarb-Bulletin, Church \& Dwight Co., In., Princeton, NJ.

56. J. R. Conner, "WERF Standby Power System Loading Analysis," WERF-EDF-0288, RW.1, October 1994.

57. Code of Federal Regulations, 10 CFR 1021, "DOE Regulations for NEPA Compliance," January 1, 1998. 
Appendix A

Mixed Waste Market Evaluation 
Table A-1. DOE Complex Inventory and Backlog of Mixed Low-Level Waste Streams Potentially Suited for WERF Incineration.

\begin{tabular}{|c|c|c|c|c|}
\hline Site Name & $\begin{array}{c}\text { Yes } \\
\text { Inventory }\end{array}$ & $\begin{array}{c}\text { Possible } \\
\text { Inventory }\end{array}$ & $\begin{array}{c}\text { Yes } \\
\text { Future } \\
\end{array}$ & Possible Future \\
\hline Argonne National Laboratory - East & 4.28 & & 4.50 & \\
\hline Battelle Columbus Laboratories & 0.51 & 0.17 & 0.18 & 0.4 \\
\hline Bettis Atomic Power Laboratory & 1.57 & & 0.72 & 0.6 \\
\hline Brookhaven National Laboratory & 18.52 & 0.003 & 0.96 & 0.0088 \\
\hline Charleston Naval Shipyard & 0.5 & 0.61 & $b$ & b \\
\hline Colonie Interim Storage Site & 2.31 & 0.014 & & \\
\hline Fernald Environmental Management Project & 303.8 & 111.8 & 60.08 & \\
\hline General Atomics & 0.004 & 1.36 & 0.32 & 0.534 \\
\hline Grand Junction Projects Office & 0.24 & & 0.04 & \\
\hline Inhalation Toxicology Research Institute & & & 5.6 & \\
\hline K-25 Site & 21.11 & 35.34 & 0.7 & 1.704 \\
\hline Knolls Atomic Power Laboratory - Kesselring & 1 & & 8.16 & 1.8 \\
\hline Knolls Atomic Power Laboratory - Schenectady & 0.9 & 0.07 & 9.2 & 3.22 \\
\hline Knolls Atomic Power Laboratory - Windsor & & & 4.6 & 15.456 \\
\hline Lawrence Livermore National laboratory & 23.1 & 68.1 & 27.6 & 1,080 \\
\hline Nevada Test Site & 0.21 & & 0.008 & \\
\hline Norfolk Naval Shipyard & 0.51 & & 22.44 & \\
\hline Oak Ridge National Laboratory & 17.151 & 2.18 & 9.68 & 3.68 \\
\hline Paducah Gaseous Diffusion Plant & 184.41 & 18.41 & & \\
\hline Pantex Plant & 73.89 & 7.11 & 97.24 & 9.67 \\
\hline Pearl Harbor Naval Shipyard & 0.05 & 5.71 & b.34 & 18.42 \\
\hline Portsmouth Gaseous Diffusion Plant & 263.12 & 670.04 & 102.8 & $1,220.4$ \\
\hline Portsmouth Naval Shipyard & & & 0.072 & 0.31 \\
\hline Princeton Plasma Physics Laboratory & & & 1.97 & \\
\hline Puget Sound Naval Shipyard & 5.32 & 16.33 & 43.28 & 15.70 \\
\hline Richland Site & 433.75 & 294.21 & $1,129.6$ & 735.7 \\
\hline RMI Titanium Company Extrusion Plant & 10.53 & 0.23 & 1.15 & 0.23 \\
\hline Rocky Flats Environmental Technology Site & 41.63 & & & \\
\hline Sandia National Laboratory - Albuquerque & 60.51 & 0.01 & 64 & \\
\hline Sandia National Laboratory - Livermore & 0.4 & & 0.8 & \\
\hline Savannah River Site & 979.9 & 17.2 & $2,143.48$ & 419.6 \\
\hline West Valley Demonstration Project & 11.44 & 0.44 & 26.31 & \\
\hline Y-12 Plant & 13.61 & 48.46 & 18.09 & 2 \\
\hline Totals & 2,474 & 1,298 & 3,784 & 3,529 \\
\hline
\end{tabular}


Table A-2. Off-site mixed waste for incineration at WERF.

\begin{tabular}{|c|c|c|c|}
\hline Waste Stream ID & \begin{tabular}{|l} 
Description \\
\end{tabular} & Inventory $\left(\mathrm{m}^{3}\right)$ & $\begin{array}{c}\text { 5-year } \\
\text { Generation }\left(\mathrm{m}^{3}\right)\end{array}$ \\
\hline AE-W015 & Organic solvents & 1.59 & 0.21 \\
\hline AE-W030 & Combustible solids w/metals & 3.6 & 1 \\
\hline AE-W031 & Combustible solids w/organics & 0.9 & 2.2 \\
\hline AE-W034 & PPE contaminated with lead & 1.8 & 1.02 \\
\hline AF-MW-01 & Air Force Munitions Maintenance Waste & 0 & 3.2 \\
\hline BT-W001 & Oil containing heavy metals \#1 & & 0.21 \\
\hline BT-W003 & Oil containing heavy metals \#2 & & 0.21 \\
\hline BT-W018 & TCLP extraction fluid & & 0.02 \\
\hline ET-CC-01 & Waste oils & 0.032 & b \\
\hline ET-W009 & Paint chips & 14 & 2.51 \\
\hline ET-W020 & Waste & 0.15 & b \\
\hline GA-CC-01 & CA. listed wastes & 0.57 & 5.38 \\
\hline GA-W031 & Methylene $\mathrm{Cl}$ & 0.208 & 0 \\
\hline GA-W034 & Doublet 11 Alcohol and Tritium & 0.06 & 0 \\
\hline GA-W037 & Waste W/F-listed solvents & 1.04 & b \\
\hline GA-W038 & Miscellaneous liquid solvents & 0.008 & 0 \\
\hline GA-W043 & SVA organic liquid & 0.02 & 0 \\
\hline GA-W044 & Wood housing HEPA filters & 0.077 & b \\
\hline GJPO-94-017 & Waste oil sludge & 0.5 & 0.5 \\
\hline GJPO-96-017 & Misc. combustible mixed waste & 0.5 & 0.5 \\
\hline GJPO-97-030 & Activated carbon & 0.2 & 0.2 \\
\hline KA-W002 & Cutting oils and liquids & & 0.1 \\
\hline KA-W007 & Oils & b & 0.29 \\
\hline KA-W009 & Organic debris & $b$ & 2 \\
\hline KA-W013 & Organic debris w/o metals & & 0.4 \\
\hline KA-W014 & Organic sludge and particulates & 0 & 0.4 \\
\hline KA-W018 & Hg contaminated organics & 0.107 & 1 \\
\hline KK-W003 & bils & & 0.25 \\
\hline KK-W004 & Metals & b & 0.25 \\
\hline KK-W005 & Metals & & 1.06 \\
\hline KK-W008 & Organic sludges/particulates & & 0.75 \\
\hline KK-W009 & Organic debris without metals & & 0.4 \\
\hline KK-W011 & Cutting oils and liquids & & 0.4 \\
\hline KK-W014 & Hg contaminated organics & 0.04 & 0.2 \\
\hline KW-W003 & Organic debris & $b$ & 1.5 \\
\hline
\end{tabular}


Table A-2. (continued).

\begin{tabular}{|c|c|c|c|}
\hline Waste Stream ID & Description & Inventory $\left(\mathrm{m}^{3}\right)$ & $\begin{array}{c}\text { 5-year } \\
\text { Generation }\left(\mathrm{m}^{3}\right) \\
\end{array}$ \\
\hline KW-W006 & Organic sludges/particulates & & 1.6 \\
\hline KW-W008 & Chemicals & & 0.3 \\
\hline KW-W010 & Hg contaminated organics & & 0.05 \\
\hline LA-W909 & Bulk oils & 6.03 & 11.4 \\
\hline LA-W911 & Solids & 33.51 & 26.2 \\
\hline LA-W912 & Cumbustible debris & 14.1 & 1.4 \\
\hline LA-W929 & Items & 14.24 & 0 \\
\hline LANL-ER-1 & TA-35 Tank D\&D waste & & b \\
\hline LB-CC-116 & Organic solids & b.15 & 0.2 \\
\hline LB-CC-118 & Lab packed chemicals & 0.15 & 0.2 \\
\hline LB-CC-120 & Pump oil & 0.15 & 0.2 \\
\hline LB-CC-124 & Contaminated debris & 1.05 & 2.1 \\
\hline LB-CC-125 & Organic liquids & 0.15 & 0.2 \\
\hline LB-CC-126 & Waste containing oil & 0.15 & 0.1 \\
\hline LB-W001 & Acidic aqueous and solid lab packs & 0.553 & 1 \\
\hline LB-W004 & Labpacked & 1.61 & 4 \\
\hline LB-W007 & Scintillation fluids & 0.058 & 0.25 \\
\hline LB-W008 & Oxidizers Lab & 0.00644 & 0.11 \\
\hline LB-W009 & Solids or contaminated debris & 0.0224 & 0.2 \\
\hline LB-W124 & Vermiculite w/oil solvents & 0.394 & 0.22 \\
\hline LBNL-CC-114 & Cyanide solution & 0.15 & 0.2 \\
\hline EL-W015 & Inorganic debris & 3 & 9 \\
\hline LLNL-CC-01 & Contaminated oil & 0.75708 & 3.79 \\
\hline MD-W021 & Oil contaminated FLORCO & 3.7 & $b$ \\
\hline MD-W023 & FLORCO & 5.4 & $b$ \\
\hline MD-W024 & Trash & 1 & 0 \\
\hline MU-W001 & Mixed Low Level Waste & 1 & 2.4 \\
\hline NA-W001 & Solid waste with heavy metals & 0 & 0 \\
\hline NN-W002 & Chromate & 0.0011 & 1.7 \\
\hline NN-W001 & Cont. w/metals/listed/org. & 0 & 2.5 \\
\hline PA-F030 & Lead contaminated debris & 3.159992 & 1.68 \\
\hline PA-G001 & Flammable materials/paints & 6.18 & 0 \\
\hline PA-K038 & Spent solvent solids/wood & 9 & 0 \\
\hline PA-L038 & Soft combustible debris & 34.700041 & 0 \\
\hline PA-M038 & Soft combustible debris & 47.300031 & 9.1 \\
\hline PA-W003 & Waste mineral spirits paint waste & 8.489956 & $b$ \\
\hline
\end{tabular}


Table A-2. (continued).

\begin{tabular}{|c|c|c|c|}
\hline Waste Stream $\mathrm{D}$ & Description & Inventory $\left(\mathrm{m}^{3}\right)$ & $\begin{array}{c}\text { 5-year } \\
\text { Generation }\left(\mathrm{m}^{3}\right) \\
\end{array}$ \\
\hline PA-W003-USEC & Point waste solids & 3.001602 & b \\
\hline PH-W004 & Chromate & 0 & 0.72 \\
\hline PN-W015 & Chromate & & 0.03 \\
\hline PO-W008 & Motor cleaning solution & 0.833 & $b$ \\
\hline PO-W012 & Uranium recovery solvent & 1.25 & b.2 \\
\hline PO-w013 & Chromic closure waste & 93 & $b$ \\
\hline PO-w028 & Lab waste & 1.675 & 1.5 \\
\hline PO-w029 & Waste antifreeze & 1.665 & 0 \\
\hline PO-W040 & Acetone still bottoms & 0.462 & 0 \\
\hline PO-W048 & Gas analyzer solutions & 7.8 & $b$ \\
\hline PO-W057 & Solvent & 34.9 & 2.5 \\
\hline PO-W058 & Activated carbon sludge & 176 & 105 \\
\hline PO-W077 & Neat TCE & 2.195 & 3 \\
\hline PO-w078 & Diesel fuel, Gasoline, Kerosene & 7.304 & 5 \\
\hline PS-W001 & Organic debris with heavy metals & & 5.64 \\
\hline PX-6.1 & Debris & & 31.95 \\
\hline RF-W071-GAC & Granulated activated carbon & 28.40282982 & 142.25 \\
\hline RL-601-01 & Mixed waste debris & 16.65 & 849.6 \\
\hline RL-ALO & Organic absorbed liquids & 30 & 279 \\
\hline RL-LPO & Organic lab packs & 124 & 98 \\
\hline SA-TG-11 & Organic liquids 11: oils & 3 & 1 \\
\hline SA-TG-12 & Organic debris w/TCLP metals & 1.4574 & 1 \\
\hline SA-TG-17-A & Absorbed machine oils & 3 & 0 \\
\hline SA-TG-17-B & Septic tanks residue & 25 & $a$ \\
\hline SA-TG-18 & Contaminants & 0.2 & 0.2 \\
\hline SA-TG-7 & Cocktails & 0.2082 & 0 \\
\hline SA-TG-8/10 & Debris & 26.8542 & 15 \\
\hline WV-W003 & Organic extraction waste & 0.084 & 0.042 \\
\hline WV-W005 & Decon solution & 0.051 & 0 \\
\hline WV-W006 & Pu Scintillation (nCi/g) & 0.0039 & e \\
\hline WV-W007 & Pyridine/cyanide waste & 0.022 & 0 \\
\hline WV-W008 & Oil with mercury & 0.00009 & 0 \\
\hline WV-W009 & Methanol & 0.002 & 0.001 \\
\hline WV-W010 & Paint & 0.023 & 0.0046 \\
\hline WV-W012 & Paint w/metals & 0.038 & 0.0056 \\
\hline WV-W014 & Sr Organic waste & 0.1238 & 0.275 \\
\hline
\end{tabular}


Table A-2. (continued).

\begin{tabular}{|l|l|l|l|}
\hline Waste Stream ID & \multicolumn{1}{|c|}{ Description } & \multicolumn{1}{c|}{ 5-year } \\
Inventory $\left(\mathrm{m}^{3}\right)$ & Generation $\left(\mathrm{m}^{3}\right)$ \\
\hline WV-W016 & R\&D Toluene & 0.00024 & 0 \\
\hline WV-W017 & Tc Aqueous waste & 0.051 & 0.0375 \\
\hline WV-W018 & DU-squeeze & 0.01435 & 0 \\
\hline WV-W021 & Ignitable organic liquids & 0.038 & 0.01325 \\
\hline WV-W022 & Spent degreaser & 0.0105 & 0 \\
\hline WV-W025 & Caustic waste & 0.096 & 0 \\
\hline WV-W027 & Oxidizers & 1.61 & 0.008 \\
\hline WV-W029 & Immersion bucket solution & 0.034 & 0 \\
\hline WV-W030 & Aqueous lab waste & 0.112 & 0.01 \\
\hline WV-W032 & Ignitable chemical products & 0.00302 & 0 \\
\hline WV-W033 & Ignitable metal waste & 0.00025 & 0 \\
\hline WV-W034 & Acidic aqueous waste & 0.019 & 0.066 \\
\hline WV-W037 & Decontaminated supernatant & 0.039 & 0 \\
\hline WV-W042 & Organic sludges & 0.122 & 0 \\
\hline WV-W043 & Ignitable liquids & 0.0019 & 0.002 \\
\hline WV-W044 & Ignitable organic liquids & 0.01721 & 0 \\
\hline WV-W047 & Inorganic siudges & 0.0024 & 0 \\
\hline WV-W053 & Sodium brohydride & 0.0008 & 0.0025 \\
\hline WV-W054 & Corrosive/flammable liquids & 0.00018 & 0.00018 \\
\hline WV-W056 & Reactives & 0.0003 & 0 \\
\hline & & 812.9422118 & 1648.31763 \\
\hline & & & \\
\hline
\end{tabular}


Table A-3. On-site mixed waste for incineration at WERF.

\begin{tabular}{|c|c|c|c|}
\hline Waste Stream ID & Description & Inventory $\left(\mathrm{m}^{3}\right)$ & $\begin{array}{c}\text { 5-year } \\
\text { Generation }\left(\mathrm{m}^{3}\right) \\
\end{array}$ \\
\hline CH-ANL-111 & Experiments & b.624591 & 0.025 \\
\hline CH-ANL-142 & Operations & 14.87926977 & 0.005 \\
\hline CH-ANL-183 & Radioactive paint stripping waste & b.208197 & 0 \\
\hline $\mathrm{CH}-\mathrm{ANL}-224$ & Maintenance & 0.0984204 & 0.0999 \\
\hline CH-ANL-554 & Lead contaminated debris & 3.317619 & 0.21 \\
\hline CH-ANL-669 & Work & 0.1438452 & 0.0032 \\
\hline D-CFA-256 & Methanol Solution & 0.0870642 & 0 \\
\hline D-CFA-551 & HDEHP/Heptane extractant & 0.2384802 & 0.05 \\
\hline D-CFA-556 & Aqueous waste subject to UHCS & 0.94635 & b \\
\hline D-CFA-662 & Scintillation cocktails & 0.208197 & 0.003 \\
\hline ID-CFA-676 & Resin column media & 0.113562 & b \\
\hline D-CFA-677 & Demineralizer filter & 0.113562 & b \\
\hline ID-CFA-695 & ARA-II Septic tank solidified sludge & 1.457379 & 1.5 \\
\hline D-CFA-705 & Vermiculite with grease & 0.208197 & b.1 \\
\hline ID-CFA-734 & Xylene, aliquot 336 with perchlorate & 0.208197 & 0.005 \\
\hline ID-INL-142 & Lead contaminated debris & 78.61459997 & 0.384 \\
\hline DD-INL-213 & Asbestos & 0.82281 & b \\
\hline D-INL-289 & Misc. laboratory wastes & 0.2701122 & 0.076 \\
\hline DD-INL-299 & Sample waste & 3.6453402 & 1.26 \\
\hline ID-INL-687 & Legacy samples & 0.9681087 & 0.34 \\
\hline ID-INL-694 & Returned sampling residue & 0.1400598 & 0 \\
\hline ID-IRC-668 & Bioassay analysis waste & & 19 \\
\hline ID-PBF-550 & MLLW from repackaging & 17.86715109 & 11.4 \\
\hline ID-PBF-678 & MWSF piping and valves & 5.0140752 & b \\
\hline D-PBF-686 & Mercury contaminated rags & 0.018927 & $b$ \\
\hline D-RWM-692 & Nitrate salts & 0.416394 & 0.4 \\
\hline ID-SMC-133 & Miscellaneous lab wastes & 1.230255 & 0.88 \\
\hline D-SMC-301 & TCA still bottoms & 0.416394 & $b$ \\
\hline D-SMC-303 & Miscellaneous paint wastes & 2.08197 & 0 \\
\hline ID-SMC-305 & bils & 0.8668566 & 1 \\
\hline ID-SMC-537 & Mercury contaminated materials & 0.208197 & b \\
\hline D-SMC-696 & Legacy TCE and corrosive water & 0.037854 & 0.003785 \\
\hline ID-TAN-170 & IET liquid waste & 0.9577062 & 0 \\
\hline ID-TAN-188 & TURCO decon solution (unused) & 0.113562 & 0 \\
\hline ID-TAN-209 & TURCO decon (oxidizer) & 0.416394 & 0 \\
\hline
\end{tabular}


Table A-3. (continued).

\begin{tabular}{|l|l|l|l|}
\hline Waste Stream D & \multicolumn{1}{|c|}{ Description } & \multicolumn{1}{c|}{ 5-year } \\
\hline D-TAN-559 & GWTF and PWTU waste & 34.172976 & 2.24 \\
\hline D-TEC-111 & Cadmium contaminated solids & 2.0066772 & 0 \\
\hline D-TEC-131 & Mercury contaminated solids & 7.235552 & 0 \\
\hline D-TEC-201 & Fo02 contaminated solids & 0.156634 & 0 \\
\hline D-TEC-217 & Scrub pump radioactive oil & 0.3852582 & 0.74 \\
\hline D-TEC-301 & Liquid acid/mercury mixed waste & & 4.5 \\
\hline D-TEC-302 & Liquid high chloride corrosive MW & 4.28434536 & 7 \\
\hline D-TEC-304 & Contaminated debris & 218.1238749 & 80 \\
\hline D-TEC-306 & Doo6-D011 contaminated solids & 1.736874 & 3.78675 \\
\hline ID-TEC-307 & Contaminated laboratory residue & 0.2128185 & 0 \\
\hline D-TEC-510 & Debris treatment residue - listed & & 22 \\
\hline D-TEC-530 & Solids & 2.7127686 & 0 \\
\hline D-TRA-294 & Solvent contaminated rags & 0.208197 & 0 \\
\hline D-TRA-525 & Solvent extractants & & 0.1 \\
\hline NR-NRF-142 & Lead contaminated debris & 2.9034018 & 1.416 \\
\hline NR-NRF-517 & Oil with heavy metals & 0.208197 & 1.432 \\
\hline & & 411.3072723 & 159.959635 \\
\hline
\end{tabular}


Table A-4. EMI incinerable waste disposition map summary.

\begin{tabular}{|c|c|c|c|c|c|}
\hline Facility & Source Site & HQId & Stream Name & Units & Life Cycle Qty \\
\hline CIF Incinerator & SARS & 00526 & WSF Sort/Seg for CIF & $\mathrm{M} 3$ & 64422.4 \\
\hline CIF Incinerator & SARS & 00528 & Incinerable Low Activity Job Control Waste & M3 & 38364 \\
\hline CIF Incinerator & SARS & 01894 & Listed incinerable debris & M3 & 636.56 \\
\hline CIF Incinerator & SARS & 01895 & Char. incinerable debris & M3 & 4753 \\
\hline CIF Incinerator & SARS & 01896 & Listed aqueons liquid & $\mathrm{M} 3$ & 15.2 \\
\hline CIF Incinerator & SARS & 01897 & Listed organic liquid & M3 & 9.82 \\
\hline CIF Incinerator & SARS & 01898 & Char. organic liquid & M3 & 2098.22 \\
\hline CIF Incinerator & SARS & 02199 & Hazardous (2nd waste from AK) & M3 & 184.1 \\
\hline TSCA Incinerator & ANLE & 00289 & PCB Contaminated LLW & M3 & 70.8 \\
\hline TSCA Incinerator & BRNL & 01190 & Packaged MLLW to TSCA & $\mathrm{M} 3$ & 8.3 \\
\hline TSCA Incinerator & CEMP & 00012 & MLLW/PCB Extract (to TSCA) & M3 & 29.4 \\
\hline TSCA Incinerator & FEMP & 00088 & MLLW-Contaminated Liquids & M3 & 510 \\
\hline TSCA Incinerator & FEMP & 01257 & Extracts & M3 & 2834 \\
\hline TSCA Incinerator & FEMP & 01270 & Neutralized MLLW-Satellite Accum Area Waste & $\mathrm{M} 3$ & 266 \\
\hline ISCA Incinerator & GEAT & 01715 & Otganic Solids & M3 & 1.25 \\
\hline TSCA Incinerator & INEL & 02045 & Debris/PCB Liquids-Onsite & M3 & 2.67 \\
\hline TSCA Incinerator & INEL & 02046 & Debris/PCB Liquids-Offsite & $\mathrm{M} 3$ & 3.54 \\
\hline TSCA Incinerator & INEL & 02441 & MLLW Sludge & M3 & 5.3 \\
\hline TSCA Incinerator & LANL & 02385 & Rad PCBs Legacy & M3 & 10.9 \\
\hline TSCA Incinerator & LANL & 02386 & PCB RCRA Legacy & M3 & 0.7 \\
\hline TSCA Incinerator & LLMS & 01826 & Organics & M3 & 509.95 \\
\hline TSCA Incinerator & NVTS & 01200 & PCB Soils & M3 & 0.1 \\
\hline TSCA Incinerator & NVTS & 01201 & TTF Solvents & M3 & 0.21 \\
\hline TSCA Incinerator & ORTN & 01581 & Organic Liquids & M3 & 20883.5 \\
\hline TSCA Incinerator & ORTN & 01620 & ORR Incinerable Liquids & M3 & 17082.4 \\
\hline TSCA Incinerator & ORTN & 01621 & ORR Incinerable Solids & M3 & 7494.99 \\
\hline TSCA Incinerator & ORTN & 01984 & PCB Contaminated LLW - Solids & M3 & 70.8 \\
\hline TSCA Incinerator & ORTN & 01986 & BNL Incinerable Solids & $\mathrm{M3}$ & 8.3 \\
\hline TSCA Incinerator & ORTN & 01987 & MLLW/PCB Extract & M3 & 2.45 \\
\hline TSCA Incinerator & ORTN & 01990 & FEMP Organic Liquids & M3 & 3614 \\
\hline TSCA Incinerator & ORTN & 01991 & Debris/PCB Liquids - Offsite & M3 & 3.55 \\
\hline TSCA Incinerator & ORTN & 01992 & Organics - Liquids & M3 & 469 \\
\hline TSCA Incinerator & ORTN & 01993 & Organics - Solids & M3 & 41 \\
\hline TSCA Incinerator & ORTN & 01996 & Cotter Concentrate - Liquids & M3 & 0.76 \\
\hline TSCA Incinerator & ORTN & 01997 & Cotter Concentrate - Solids & M3 & 0.12 \\
\hline TSCA Incinerator & ORTN & 02000 & TTF Solvents - Liquids & M3 & 0.21 \\
\hline TSCA Incinerator & ORTN & 02001 & PCB Soils - Solids & M3 & 0.1 \\
\hline TSCA Incinerator & ORTN & 02002 & BAF, BBU, BBN, BAO, BAA - Liquids & M3 & 350.26 \\
\hline TSCA Incinerator & ORTN & 02003 & BAN, BAL-Solids & M3 & 1045 \\
\hline TSCA Incinerator & ORTN & 02004 & AAG, AAH - Liquids & M3 & 2353 \\
\hline TSCA Incinerator & ORTN & 02005 & AAK, AAX - Solids & M3 & 6308 \\
\hline TSCA Incinerator & ORTN & 02006 & Other Liquids & M3 & 0.68 \\
\hline TSCA Incinerator & ORTN & 02007 & PCB Organic Liquids & M3 & 127 \\
\hline TSCA Incinerator & ORTN & 02008 & PCB Oxganic Solids & M3 & 17 \\
\hline TSCA Incinerator & ORTN & 02010 & Incinerable RAD PCBs - Liquids & M3 & 0.23 \\
\hline
\end{tabular}


Table A-4. (continued).

\begin{tabular}{|c|c|c|c|c|c|}
\hline Facility & Source Site & HQ Id & Stream Name & Units & Life Cycle Qty \\
\hline TSCA Incinerator & ORTN & 02011 & Incinerable RAD PCBs - Solids & M3 & 0.8 \\
\hline TSCA Incinerator & ORTN & 02012 & PCB Wastes Liquids/Solids & M3 & 0.43 \\
\hline TSCA Incinerator & ORMN & 02013 & PCB Wastes Liquids/Solids & $\mathrm{M3}$ & 6.26 \\
\hline TSCA Incinerator & ORTN & 02014 & Organic Solids & $\mathrm{M3}$ & 125 \\
\hline TSCA Incinerator & ORTN & 02406 & Other Liquids & $\mathrm{M3}$ & 5.32 \\
\hline TSCA Incinerator & ORTN & 02408 & Debris/PCB Liquids - Onsite & M3 & 2.67 \\
\hline TSCA Incinerator & PGDP & 00434 & RPCB Liguid & M3 & 95 \\
\hline TSCA Incinerator & PGDP & 00439 & LLW Liquids & M3 & 110 \\
\hline TSCA Incinerator & PGDP & 00445 & RPCB Soft Solids & M3 & 917 \\
\hline TSCA Incinerator & PGDP & 00447 & MLLW Soft Solids - Incinerable & M3 & 128 \\
\hline TSCA Incinerator & PGDP & 00448 & MLLW Liquids - Incinerable (to TSCA) & M3 & 32 \\
\hline TSCA Incinerator & PGDP & 00460 & Suspect HAZ Liquids & M3 & 8.04 \\
\hline TSCA Incinerator & PGDP & 00468 & Suspect MLLW Liquids & M3 & 75.19727 \\
\hline TSCA Incinerator & PORT & 00474 & TSCA Liquids & M3 & 1914 \\
\hline TSCA Incinerator & PORT & 00475 & Mixed Incinerable Liquids & M3 & 539 \\
\hline TSCA Incinerator & PORT & 00478 & TSCA Soft Solids & M3 & 1871 \\
\hline TSCA Incinerator & PORT & 01979 & Incinerable Solids (to TSCA) & M3 & 4437 \\
\hline TSCA Incinerator & RFTS & 01405 & PCB Organic Liquids & M3 & 127 \\
\hline TSCA Incinerator & RFTS & 02405 & PCB Organic Solids & M3 & 17 \\
\hline TSCA Incinerator & SARS & 01905 & Incinerable rad PCBs & M3 & 8.5 \\
\hline TSCA Incinerator & SNLN & 00617 & Septage Liquids & M3 & 15 \\
\hline TSCA Incinerator & WVDP & 00650 & PCB Liquid Wastes & M3 & 6.64 \\
\hline WERF Incinerator & ANLE & 01164 & Sorted Mixed Waste to WERF & $\mathrm{M3}$ & 17.32 \\
\hline WERF Incinerator & ETEC & 01682 & Analytical Lab Waste & M3 & 0.15 \\
\hline WERF Incinerator & HASI & 01515 & WERF Feed & M3 & 16.65 \\
\hline WERF Incinerator & LABL & 01748 & Aqueous Liquids (INEEL FY98 - 03) & M3 & 3.66 \\
\hline WERF Incinerator & LABL & 01750 & $\begin{array}{l}\text { Lab-Packed Oxidizers, Debris, Vermiculite with Oil } \\
\text { (INEEL) }\end{array}$ & M3 & 2.08 \\
\hline WERF Incinerator & LABL & 01752 & Organic Liquids/Scint. Vials (INEEL) & M3 & 4.49 \\
\hline WERF Incinerator & LANL & 02383 & Organic Combustible Solids Newly Generated (post 1998) & M3 & 75 \\
\hline WERF Incinerator & LANL & 02384 & Organic Combustible Solids Legacy (pre 1999) & M3 & 100 \\
\hline WERF Incinerator & LLMS & 01828 & Debris & M3 & 179 \\
\hline WERF Incinerator & PAPL & 01351 & MLLW From Ops (to WERF) & M3 & 50 \\
\hline WERF Incinerator & PGDP & 00441 & MLLW Soft Solids - Incinerable & M3 & 128 \\
\hline WERF Incinerator & PGDP & 00446 & MLLW Liquids - Incinerable (to WERF) & M3 & 32 \\
\hline WERF Incinerator & PORT & 01980 & Incinerable Solids (to WERF) & M3 & 150 \\
\hline WERF Incinerator & RFTS & 01406 & Combustible Solids & M3 & 20.6 \\
\hline WERF Incinerator & SNLN & 00599 & HDRV - WERF \#2 & M3 & 12.2 \\
\hline WERF Incinerator & SNLN & 00613 & Organic Debris & M3 & 67.7 \\
\hline WERF Incinerator & SNLN & 00614 & Organic Liquids & M3 & 5.1 \\
\hline WERF Incinerator & SNLN & 00615 & Organic Debris with TCLP & M3 & 2.2 \\
\hline WERF Incinerator & SNLN & 00616 & Septage > IDR & $\mathrm{M3}$ & 15 \\
\hline
\end{tabular}




\begin{tabular}{|c|c|c|c|c|c|c|}
\hline $\mathrm{Cl}$ & \multicolumn{2}{|r|}{$\mathrm{Hg}$} & \multicolumn{2}{|c|}{$\mathrm{Pb}$} & \multicolumn{2}{|c|}{$\mathrm{Cd}$} \\
\hline Est. mass $(\mathrm{lb})$ & $\begin{array}{c}\text { Avg conc } \\
\text { (ppmw) }\end{array}$ & Est. mass $(\mathrm{lb})$ & $\begin{array}{l}\text { Avg conc } \\
\text { (ppmw) }\end{array}$ & Est. mass (lb) & $\begin{array}{c}\text { Avg conc } \\
\text { (ppmw) }\end{array}$ & Est. mass $(\mathrm{lb})$ \\
\hline 7 & 0 & 0 & 0 & 0 & 19 & 0.4 \\
\hline 27 & 0 & 0 & 0 & 0 & 0 & 0 \\
\hline 127 & 11 & 5 & 45 & 19 & 58 & 25 \\
\hline 945 & 11 & 36 & 45 & 144 & 58 & 187 \\
\hline 30 & 540 & 19 & 540 & 19 & 1,620 & 56 \\
\hline 13 & 0 & 0 & 0 & 0 & 0 & 0 \\
\hline 2,783 & 0.4 & 2 & 0 & 0 & 0 & 0 \\
\hline 3,931 & 7 & 61 & 22 & 182 & 33 & 269 \\
\hline 7,911 & 11 & 301 & 45 & 1,206 & 58 & 1,569 \\
\hline 13,285 & 11 & 505 & 45 & 2,025 & 58 & 2,634 \\
\hline 21,196 & 11 & 806 & 45 & 3,230 & 58 & 4,203 \\
\hline 38 & 11 & 1 & 45 & 6 & 58 & 8 \\
\hline 25,165 & 11 & 868 & 42 & 3,418 & 55 & 4,480 \\
\hline
\end{tabular}


Table A-5. Estimated waste streams that may be treated at CIF.

\begin{tabular}{|c|c|c|c|c|c|}
\hline Generator & Waste Description & $\begin{array}{l}\text { Waste } \\
\text { Type }\end{array}$ & $\operatorname{Vol}\left(\mathrm{m}^{3}\right)$ & $\begin{array}{c}\text { Est. Mass } \\
\text { (lb) }\end{array}$ & $\begin{array}{r}\text { Avg I } \\
\text { (ppi } \\
\end{array}$ \\
\hline SNL & Organic debris & $S$ & 34 & 23,159 & 288 \\
\hline SRS & Organic liquid & $L$ & 20.4 & 43,216 & 626 \\
\hline SRS & Listed incinerable debris & $\mathrm{s}$ & 636.56 & 433,600 & 292 \\
\hline SRS & Char. Incinerable debris & S & 4,753 & $3,237,556$ & 292 \\
\hline SRS & Listed aqueous liquid & $\mathrm{L}$ & 15.2 & 34,814 & 854 \\
\hline \multirow[t]{2}{*}{ SRS } & Listed organic liquid & $L$ & 9.82 & 20,803 & 626 \\
\hline & Char. Organic liquid & $L$ & $2,098.22$ & $4,444,935$ & 626 \\
\hline SRS & MLLW TOTALS: & & 7,567 & $8,238,084$ & 477 \\
\hline \multirow[t]{2}{*}{ SRS } & Incinerable low activity job control waste & $S$ & 38,364 & $27,092,657$ & 292 \\
\hline & Low activity bulk waste & $S$ & $64,422.4$ & $45,495,099$ & 292 \\
\hline \multirow[t]{3}{*}{ SRS } & LLW TOTALS: & & 102,786 & $72,587,756$ & 292 \\
\hline & Hazardous & $S$ & 184.1 & 130,011 & 292 \\
\hline & CIF TOTALS: & & 110,538 & $80,955,851$ & 311 \\
\hline
\end{tabular}


Table A-6. Estimated waste streams that may be treated at the TSCA incinerator.

\begin{tabular}{|c|c|c|c|c|c|}
\hline Generator & Waste Description & Waste type & Vol. $\left(\mathrm{m}^{3}\right)$ & $\begin{array}{c}\text { Est. mass } \\
\text { (lb) }\end{array}$ & $\begin{array}{l}\text { avg c) } \\
\text { (pprs) }\end{array}$ \\
\hline ANL-E & PCB contaminated LLW & $S$ & 70.8 & 49,999 & 56,55 \\
\hline BATELLE & MLLW/PCB-contaminated rubble/debris & $S$ & 29.4 & 20,762 & 285,6 \\
\hline BROOKHAVEN & Packaged MLLW TSCA debris & $S$ & 8.3 & 5,861 & 285,6 \\
\hline FERNALD & MLLW-contaminated liquids & $\mathbf{L}$ & 510 & $1,113,003$ & 19,63 \\
\hline FERNALD & $\begin{array}{l}\text { Extracts: MLLW/PCB-cont. sludge; MLLW/PCB } \\
\text { \& F-listed cont. soils; MLLW-cont. debris TSAA; } \\
\text { All sent thru organic solvent extraction process }\end{array}$ & L & 2,834 & $4,997,823$ & 178,5 \\
\hline FERNALD & Neutralized MLLW-SAA waste & $\underline{L}$ & 266 & 527,734 & 19,63 \\
\hline GEN. ATOMICS & Organic solids & $S$ & 1.248 & 1,560 & 8,337 \\
\hline INEEL & Debris/PCB liquids/Sludges - Onsite & sludge & 2.67 & 4,709 & 113,7 \\
\hline INEEL & Debris/PCB liquids/Sludges - Offsite & sludge & 3.54 & 6,243 & 113,7 \\
\hline INEEL & MLLW sludge & sludge & 5.3 & 9,931 & 77,44 \\
\hline INEEL & PCB contaminated lab residue & $s$ & 0.0957 & 168 & 6,817 \\
\hline INEEL & PCB contaminated waste & $S$ & 1.6487 & 1,164 & 56,35 \\
\hline LANL & Rad PCBs & $\mathrm{L}$ & 10.9 & 23,788 & $56,35^{5}$ \\
\hline LANL & PCB TSCA & $\mathrm{L}$ & 0.7 & 1,528 & 56,35 \\
\hline LLNL & Organics & $s$ & 509.95 & 981,370 & 23,11 \\
\hline NTS & PCB soils & $s$ & 0.1 & 300 & 111 \\
\hline NTS & TTF solvents & $\mathrm{L}$ & 0.21 & 469 & $38,42 !$ \\
\hline ORNL & ORR incinerable liquids & $\mathrm{L}$ & $17,082.4$ & $37,656,499$ & 22,14 \\
\hline ORNL & ORR incinerable solids & $s$ & $7,494.99$ & $5,292,962$ & 138,8 \\
\hline ORNL & MLLW soft solids from OR ER & $S$ & 207 & 146,183 & 110,2 \\
\hline PADUCAH & MLLW soft solids & $S$ & 128 & 90,394 & $110,2 !$ \\
\hline PADUCAH & MLLW liquids & $L$ & 32 & 70,894 & $19,63 t$ \\
\hline PADUCAH & RPCB liquid & $L$ & 95 & 210,465 & 43,88 \\
\hline PADUCAH & RPCB soft solids & $S$ & 917 & 647,585 & 109,9 \\
\hline PADUCAH & Suspected MLLW liquids & $\mathrm{L}$ & 75 & 166,157 & 19,63 \\
\hline PORTSMOUTH & TSCA liquids & $\mathbf{L}$ & $1,914.4$ & $3,924,702$ & 43,88 \\
\hline PORTSMOUTH & Mixed waste incinerable liquids & $\mathrm{L}$ & 539 & $1,105,001$ & 22,14 \\
\hline
\end{tabular}


Table A-6. (continued).

\begin{tabular}{|c|c|c|c|c|c|}
\hline Generator & Waste Description & Waste type & Vol. $\left(\mathrm{m}^{3}\right)$ & $\begin{array}{c}\text { Est. mass } \\
\text { (lb) }\end{array}$ & $\begin{array}{l}\underset{\text { avg }}{\text { (ppi }} \\
\text { (n) }\end{array}$ \\
\hline PORTSMOUTH & TSCA soft solids & $S$ & 1,871 & $1,321,300$ & 109, \\
\hline PORTSMOUTH & Sorted MW incinerable soft solids & $S$ & 4,437 & $3,133,409$ & 138, \\
\hline ROCKY FLATS & PCB organic liquids & $\mathrm{L}$ & 127 & 258,962 & $24,4^{\prime}$ \\
\hline ROCKY FLATS & PCB organic solids & $S$ & 17 & 21,248 & $8,33^{\prime}$ \\
\hline SNL & Septage liquids & $\mathrm{L}$ & 15 & 33,066 & 12,1 \\
\hline SRS & Incinerable rad. PCB & $\mathrm{S}$ & 1 & 681 & $56,3:$ \\
\hline WEST VALLEY & PCB wastes liquids/solids & $S$ & 6.63 & 10,231 & 56,3: \\
\hline $\mathrm{BCLJ}$ & WS code 1987 - MLLW & $\mathrm{L}$ & 2.45 & 5,347 & $\mathrm{ND}$ \\
\hline MOPL & WS code 1996 - MLLW & $\mathrm{L}$ & 0.76 & 1,659 & ND \\
\hline MOPL & WS code 1997 - MLLW & $\mathrm{L}$ & 0.12 & 262 & $\mathrm{ND}$ \\
\hline REME & WS code 2006 - MLLW & $\mathrm{L}$ & 0.68 & 1,484 & ND \\
\hline \multirow[t]{2}{*}{ REME } & WS code 2406 - MLLW & $\mathrm{L}$ & 5.32 & 11,610 & ND \\
\hline & MLLW TOTALS: & & 39,224 & $61,856,513$ & $55,3 i$ \\
\hline ORNL & Organic liquids & $\mathrm{L}$ & 20,883 & $46,034,554$ & $24,4:$ \\
\hline \multirow[t]{2}{*}{ PADUCAH } & LLW liquid & $\mathrm{L}$ & 110 & 243,697 & 19,6: \\
\hline & LLW TOTALS: & & 20,993 & $46,278,251$ & 24,4 \\
\hline \multirow[t]{2}{*}{ PADUCAH } & Suspected HAZ liquids & $\mathrm{L}$ & 8 & 17,723 & 19,6: \\
\hline & TSCA Incinerator TOTALS: & & 60,225 & $108,152,488$ & 42,1: \\
\hline
\end{tabular}




\begin{tabular}{lllll}
\hline $\mathrm{Cl}$ & $\mathrm{Hg}$ & $\mathrm{Pb}$ & $\mathrm{Cd}$ & PCBs \\
\hline
\end{tabular}

\begin{tabular}{|c|c|c|c|c|c|c|c|c|}
\hline $\begin{array}{l}\text { Est. mass } \\
\text { (b) }\end{array}$ & $\begin{array}{c}\text { avg conc } \\
\text { (ppmw) }\end{array}$ & $\begin{array}{c}\text { Est. mass } \\
\text { (lb) }\end{array}$ & $\begin{array}{c}\text { avg conc } \\
\text { (ppmw) }\end{array}$ & $\begin{array}{c}\text { Est. mass } \\
\text { (lb) }\end{array}$ & $\begin{array}{l}\text { avg conc } \\
\text { (ppmw) }\end{array}$ & $\begin{array}{c}\text { Est. mass } \\
(\mathrm{lb})\end{array}$ & $\begin{array}{l}\text { avg conc } \\
\text { (ppmw) }\end{array}$ & $\begin{array}{c}\begin{array}{c}\text { Est. mass } \\
(\mathrm{lb})\end{array} \\
\end{array}$ \\
\hline 145,285 & 17 & 22 & 1,163 & 1,537 & 122 & 161 & 55,200 & 72,935 \\
\hline 435,168 & 8 & 25 & 1,134 & 3,554 & 237 & 742 & 100,464 & 314,795 \\
\hline 6,328 & 0.6 & 0.2 & 27 & 7 & 1.0 & 0.3 & 12,819 & 3,320 \\
\hline 177 & 0.5 & 0.01 & 389 & 8 & 8 & 0.2 & 843 & 18 \\
\hline 400 & 0.9 & 0.03 & 10 & 0.3 & 0.5 & 0.017 & 11,793 & 390 \\
\hline 38 & 2 & 0.0014 & 139 & 0.095 & 22 & 0.015 & 47,215 & 32 \\
\hline 577 & 2 & 0.02 & 139 & 1 & 22 & 0.2 & 47,215 & 483 \\
\hline ND & $\mathrm{ND}$ & ND & $\mathrm{ND}$ & ND & $\mathrm{ND}$ & $\mathrm{ND}$ & ND & $\mathrm{ND}$ \\
\hline $\mathrm{ND}$ & ND & ND & $\mathrm{ND}$ & $\mathrm{ND}$ & $\mathrm{ND}$ & $\mathrm{ND}$ & ND & $\mathrm{ND}$ \\
\hline $\mathrm{ND}$ & $\mathrm{ND}$ & ND & ND & ND & $\mathrm{ND}$ & ND & $\mathrm{ND}$ & ND \\
\hline ND & $\mathrm{ND}$ & ND & $\mathrm{ND}$ & ND & ND & $\mathrm{ND}$ & $\mathrm{ND}$ & ND \\
\hline ND & ND & ND & ND & ND & $\mathrm{ND}$ & $\mathrm{ND}$ & $\mathrm{ND}$ & ND \\
\hline $3,426,154$ & 3 & 200 & 325 & 20,076 & 51 & 3,160 & 37,874 & $2,342,756$ \\
\hline $1,124,866$ & 0.6 & 30 & 27 & 1,232 & 1.0 & 45 & 12,819 & 590,110 \\
\hline 4,786 & 0.8 & 0.2 & 21 & 5 & 0.8 & 0.2 & 10,830 & 2,639 \\
\hline $1,129,651$ & 0.65 & 30 & 27 & 1,237 & 0 & 46 & 0.001 & 592,749 \\
\hline 348 & 0.8 & 0.01 & 21 & 0.4 & 0.8 & 0.015 & 10,830 & 192 \\
\hline $4,556,153$ & 2 & 231 & 197 & 21,314 & 30 & 3,206 & 27,144 & $2,935,697$ \\
\hline
\end{tabular}


Table A-7. Estimated waste streams that may be treated at WERF.

\begin{tabular}{|c|c|c|c|c|c|}
\hline Generator & Waste Description & $\begin{array}{c}\text { Waste } \\
\text { type }\end{array}$ & Vol. $\left(\mathrm{m}^{3}\right)$ & $\begin{array}{c}\text { Est. Mass } \\
\text { (lb) }\end{array}$ & $\begin{array}{r}\text { Avg co } \\
\text { (ppme }\end{array}$ \\
\hline ANL-E & Sorted mixed waste & $S$ & 17.32 & 12,231 & 15,905 \\
\hline ETEC & Paint chips from ER & $S$ & 16.65 & 42,330 & 9,087 \\
\hline ETEC & Analytical lab waste & $S$ & 0.15 & 70 & 1,001 \\
\hline HANFORD & Sorted organic solids & $S$ & 16.65 & 36,667 & 46,912 \\
\hline INEEL & Debris/sludges/liquids/lab packs & $S$ & 29.3445 & 41,076 & 19,963 \\
\hline INEEL & From HLW & $S$ & 7.673 & 6,773 & $\mathrm{ND}$ \\
\hline INEEL & WERF sized MLLW & S & 134.22 & 284,358 & 313 \\
\hline INEEL & ARA-II septic tank solidified sludge & Sludge & 1.4373 & 3,083 & 132 \\
\hline INEEL & Nitrate residue & $S$ & 0.4164 & 634 & 3,174 \\
\hline INEEL & Legacy TCE and corrosive water & $\mathrm{L}$ & 0.0379 & 75 & ND \\
\hline GJPO & Waste oil sludge & Sludge & 0.5 & 1,011 & 213,400 \\
\hline GJPO & Miscellaneous combustible waste & $S$ & 0.5 & 388 & 35,993 \\
\hline GJPO & Activated carbon & $\mathbf{s}$ & 0.2 & 212 & 6,002 \\
\hline LANL & Organic combustible solids (new) & $\mathbf{S}$ & 75 & 49,599 & 46,912 \\
\hline LANL & Organic combustible solids (legacy) & $S$ & 100 & 66,132 & 46,912 \\
\hline LBNL & Aqueous liquids (INEEL FY98-03) & $\mathrm{L}$ & 3.66 & 8,068 & 186 \\
\hline LBNL & $\begin{array}{l}\text { Lab-packed oxidizers, debris, vermiculite with } \\
\text { oil }\end{array}$ & $S$ & 2.08 & 1,542 & 18,537 \\
\hline LBNL & Organic liquids/scintillation vials & $\mathrm{L}$ & 4.49 & 8,928 & 189,903 \\
\hline LLNL & Debris & $S$ & 237 & 255,997 & $35,445^{\prime}$ \\
\hline PADUCAH & MLLW soft solids & S & 128 & 90,394 & 19,151 \\
\hline PADUCAH & MLLW liquids & L & 32 & 70,894 & 48,633 \\
\hline PANTEX & MLLW from operations & $\underline{S}$ & 50 & 44,138 & 45,748 \\
\hline PORTS-MOUTH & Sorted mixed waste incinerable soft solids & $S$ & 150 & 105,930 & 19,151 \\
\hline RFP & Combustible solids & $\mathbf{S}$ & 21 & 11,864 & 35,993 \\
\hline SNL & Organic debris w/TCLP & $S$ & 2.2 & 2,750 & 34,721 \\
\hline SNL & Organic liquid & $\mathrm{L}$ & 5.1 & 10,141 & 213,400 \\
\hline SNL & Septage > LDR & $\mathrm{L}$ & 15 & 33,066 & 132 \\
\hline SNL & MLLW liquids incinerable & $\mathrm{L}$ & 32 & 70,541 & 48,633 \\
\hline SNL & Suspect MLLW liquids & $\mathrm{L}$ & 75.2 & 165,771 & 48,633 \\
\hline
\end{tabular}


Table A-7. (continued).

\begin{tabular}{l|l|l|l|l|l|l}
\hline \multicolumn{1}{c|}{ Generator } & \multicolumn{1}{|c|}{ Waste Description } & & & & & \\
\hline COMM & WS code 2076-MLLW & $\begin{array}{c}\text { Waste } \\
\text { type }\end{array}$ & Vol. $\left(\mathrm{m}^{3}\right)$ & $\begin{array}{c}\text { Est. Mass } \\
(\mathrm{b})\end{array}$ & $\begin{array}{c}\text { Avg } \\
(\mathrm{ppn}\end{array}$ \\
\hline NRF & WS code 929 -MLLW & & $\mathrm{S}$ & 21.6 & 15,254 & 17,196 \\
\hline & MLLW TOTALS: & & $\mathrm{S}$ & 1.03 & 727 & 17,196 \\
\hline ANL-W & Photo-remediation residuals & & & 1180.46 & $1,440,644$ & 30,094 \\
\hline SNL & Organic debris & $\mathrm{S}$ & 370 & 261,294 & 209,21 \\
\hline SNL & HDRV \#2 & $\mathrm{S}$ & 67.7 & 84,618 & 34,721 \\
\hline & LLW TOTALS: & L & & 12.2 & 8,616 & 17,196 \\
\hline & WERF TOTALS: & & & 1,630 & $1,795,172$ & 56,323
\end{tabular}




\begin{tabular}{|c|c|c|c|c|c|c|c|c|}
\hline \multicolumn{2}{|l|}{$\mathrm{Cl}$} & \multicolumn{3}{|c|}{$\mathrm{Hg}$} & \multicolumn{2}{|c|}{$\mathrm{Pb}$} & \multicolumn{2}{|c|}{$\mathrm{Cd}$} \\
\hline $\begin{array}{c}\text { Est. mass } \\
(\mathrm{lb})\end{array}$ & $\begin{array}{c}\%>500 \\
\mathrm{ppm}^{1}\end{array}$ & $\begin{array}{l}\text { avg. conc } \\
\text { (ppmw) }\end{array}$ & $\begin{array}{c}\text { Est. mass } \\
(\mathrm{lb})\end{array}$ & $\begin{array}{c}\%>0.2 \\
\mathrm{ppm}^{1}\end{array}$ & $\begin{array}{c}\text { avg conc } \\
\text { (ppmw) }\end{array}$ & $\begin{array}{l}\text { Est. mass } \\
\text { (lb) }\end{array}$ & $\begin{array}{l}\text { avg conc } \\
\text { (ppmw) }\end{array}$ & \begin{tabular}{|l} 
Est. mass \\
(lb)
\end{tabular} \\
\hline 262 & & 40 & 0.6 & & 1,218 & 19 & 1,566 & 24 \\
\hline 13 & & 40 & 0.03 & & 1,218 & 0.9 & 1,566 & 1 \\
\hline 43,355 & & 83 & 119 & & 3,432 & 4,944 & 194 & 279 \\
\hline 54,667 & 18 & 0.015 & 0.004 & 0 & 3 & 0.8 & 0.2 & 0.1 \\
\hline 2,938 & 100 & 3 & 0.3 & 24 & 6,280 & 531 & 98 & 8 \\
\hline 148 & & 40 & 0.3 & & 1,218 & 10 & 1,566 & 13 \\
\hline 57,753 & & 0.0001 & 0.6 & & 0.2 & 543 & 0.02 & 22 \\
\hline 101,109 & & 67 & 120 & & 3,056 & 5,486 & 167 & 301 \\
\hline
\end{tabular}


Table A-8. Estimated wastestreams that could be treated at WERF if upgraded and CIF and TSCA were not upgraded

\begin{tabular}{|c|c|c|c|c|c|c|c|c|}
\hline & & & & & \multicolumn{4}{|c|}{$\mathrm{Cl}$} \\
\hline Generator & $\begin{array}{l}\text { Planned } \\
\text { receiver }\end{array}$ & Waste Description & Vol $\left(\mathrm{m}^{3}\right)$ & $\begin{array}{c}\text { Est mass } \\
\text { (lb) }\end{array}$ & $\begin{array}{l}\text { avg conc } \\
\text { (ppmw) }\end{array}$ & $\begin{array}{c}\text { Est mass } \\
\text { (lb) }\end{array}$ & $\begin{array}{c}\text { Cons. DRF } \\
\text { partial }\end{array}$ & \\
\hline SNL & CFF & Organic debris & 34 & 23.159 & 288 & 6.7 & 1 & \\
\hline SNL & CIF & Organic liquid & 20.4 & 43,216 & 626 & 27 & 1 & \\
\hline SRS & $\mathrm{CIF}$ & Listed incinerable debris & 636.56 & 433.600 & 292 & 127 & 1 & \\
\hline SRS & $\mathrm{CFF}$ & Char. Incinerable debris & 4,753 & $3,237,556$ & 292 & 945 & 1 & \\
\hline SRS & CIF & Listed aqueous liquid & 15.2 & 34,814 & 854 & 30 & 1 & \\
\hline SRS & CIF & Listed organic liquid & 9.82 & 20.803 & 626 & 13 & 1 & 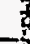 \\
\hline SRS & CIF & Char. Organic liquid & $2,098.22$ & $4,444,935$ & 626 & 2,783 & 1 & \\
\hline SRS & CIF & Iricin. low activity job control waste & 38,364 & $27,092,657$ & 292 & 7,911 & 1 & \\
\hline SRS & CIF & Low activity bulk waste & 64.422 .4 & 45.495 .099 & 292 & 13.285 & 1 & 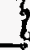 \\
\hline$\underline{\text { SRS }}$ & CIF & Hazardous & 184.1 & 130.011 & 292 & 38 & 1 & 8 \\
\hline & & CIF TOTALS: & 110,538 & $80,955,851$ & & 25,165 & & \\
\hline ANLE & TSCA & PCB contaminated LLW & 70.8 & 146,183 & 56,357 & 8,238 & 2.25 & 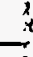 \\
\hline BATELLE & TSCA & MLLW/PCB-cont nubble/debris & 29.4 & 90.394 & 285.601 & 25.817 & 11.42 & \\
\hline BROOK. & TSCA & Pkgd MOLW TSCA debris & 8.3 & 70.894 & 285,601 & 20.247 & 11.42 & \\
\hline FERNALD & TSCA & MLLW-contaminated liquids & 510 & 210.465 & 19.638 & 4,133 & 1 & \\
\hline FERNALD & $\mathrm{TSCA}$ & $\begin{array}{l}\text { Extracts: MWW/PCB-cont. sludge; MLLW/PCB \& } \\
\text { F-listed cont soils; MILIW-cont. debris TSAA; All sent } \\
\text { thru org. solvent extraction proc. }\end{array}$ & 2,834 & 647,585 & 178,583 & 115,648 & 7.14 & \\
\hline FERNALD & ISCA & Neutralized MLLW-SAA waste & 266 & 17.723 & $\mathrm{ND}$ & & ND & \\
\hline GA & $\mathrm{TSCA}$ & Organic solids & 1.248 & 166,157 & 8,337 & 1.385 & 1 & \\
\hline NEEL & $\mathrm{ISCA}$ & Debris/PCB liguids/Sludges - Onsite & 2.67 & 243,697 & 113,792 & 27,731 & 4.55 & 1 \\
\hline NEEL & TSCA & Debrie/PCB liq/Sludges-Offsite & 3.54 & $3,924.702$ & 113.792 & 446.599 & 4.55 & \\
\hline NEEEL & $\mathrm{TSCA}$ & MLLW sludge & 53 & 1.105 .001 & 77.441 & 85.572 & 3.10 & \\
\hline INEEL & TSCA & PCB cont lab residue & 0.0957 & 1.321 .300 & 6.817 & 9.007 & 1 & \\
\hline NEEL & $\mathrm{TSCA}$ & PCB contaminated waste & 1.6487 & $3,133.409$ & 56,357 & 176,589 & 225 & $i$ \\
\hline LANL & TSCA & Rad PCBs & 10.9 & 258.962 & 56,357 & 14,594 & 2.25 & \\
\hline LANL & TSCA & PCB TSCA & 0.7 & 21,248 & 56,357 & 1,197 & 2.25 & \\
\hline INL & TSCA & Organios & 509.95 & 33,066 & 23,110 & 764 & 1 & \\
\hline NTS & TSCA & PCB soils & 0.1 & 681 & 56.357 & 38.388 & 2.25 & \\
\hline NTS & TSCA & TTF solvents & 0.21 & 10,231 & 38.425 & 393 & 1.54 & \\
\hline ORNL & TSCA & Organic liquids & 20,883 & 5.347 & 24,435 & 131 & 1 & \\
\hline ORNL & $\mathrm{TSCA}$ & ORR incinerable liquids & $17,082.4$ & 1,659 & 22,143 & 36.726 & 1 & \\
\hline ORNL & $\mathrm{TSCA}$ & ORR incinerable solids & $7,494.99$ & 262 & 138,880 & 36.370 & 5.56 & \\
\hline ORNL & TSCA & From OR ER (GAW) & 207 & 1,484 & ND & $\mathrm{ND}$ & $\mathrm{ND}$ & \\
\hline PADUCAH & TSCA & MLLW soft solids & 128 & 11.610 & 110.262 & 1,280 & 4.41 & \\
\hline PADUCAH & $\mathrm{TSCA}$ & MOLW liquids & 32 & 108.152 .488 & 19.638 & 2.123 .883 & 1 & \\
\hline PADUCAH & $\mathrm{TSCA}$ & RPCB liquid & 95 & 0 & 43.886 & 0.000 & 1.76 & \\
\hline PADUCAH & TSCA & RPCB soft solids & 917 & 0 & 109,956 & 0.000 & 4.40 & .t. \\
\hline PADUCAH & TSCA & Suspected HAZ liquids & 8 & 0 & 19,638 & 0.000 & 1 & ' \\
\hline PADUCAH & TSCA & Suspected MLLW liquids & 75 & 0 & 19,638 & 0.000 & 1 & \\
\hline PADUCAH & TSCA & LLW liquid & 110 & 10 & 19,638 & 0.000 & 1 & \\
\hline
\end{tabular}


Table A-8. (continued).

\begin{tabular}{|c|c|c|c|c|c|c|c|}
\hline & & & & & \multicolumn{3}{|c|}{$\mathrm{Cl}$} \\
\hline Generator & $\begin{array}{l}\text { Planned } \\
\text { receiver }\end{array}$ & Waste Description & Vol. $\left(\mathrm{m}^{3}\right)$ & $\begin{array}{c}\begin{array}{c}\text { Est. mass } \\
\text { (b) }\end{array} \\
\end{array}$ & $\begin{array}{l}\text { avg conc } \\
\text { (ppmw) }\end{array}$ & $\begin{array}{c}\begin{array}{c}\text { Est. mass } \\
\text { (b) }\end{array} \\
\end{array}$ & $\begin{array}{r}\text { Cons. D } \\
\text { partial }\end{array}$ \\
\hline PORTS. & $\mathrm{ISCA}$ & TSCA liquids & $1,914.4$ & 0 & 43,886 & 0.000 & 1.76 \\
\hline PORTS. & ISCA & MW incinerable liquids & 539 & 0 & 22,143 & 0.000 & 1 \\
\hline PORTS. & TSCA & TSCA soft solids & 1,871 & 0 & 109,956 & 0.000 & 4.40 \\
\hline PORTS. & TSCA & Sorted MW incin. soft solids & 4,437 & 0 & 138,880 & 0.000 & 5.56 \\
\hline RF & TSCA & PCB organic liguids & 127 & 0 & 24,435 & 0.000 & 1 \\
\hline RF & TSCA & PCB organic solids & 17 & 0 & 8,337 & 0.000 & 1 \\
\hline SNL & TSCA & Septage liquids & 15 & 0 & 12,110 & 0.000 & 1 \\
\hline SRS & TSCA & Incinerable rad. PCB & 1 & 0 & 56,357 & 0.000 & 2.25 \\
\hline W.VALLEY & TSCA & PCB wastes liquids/solids & 6.63 & 0 & 56,357 & 0.000 & 2.25 \\
\hline $\mathrm{BCLN}$ & TSCA & WS code 1987 - MLLW & 2.45 & 0 & $\mathrm{ND}$ & ND & ND \\
\hline MOPL & TSCA & WS code 1996 - MLLW & 0.76 & 0 & $\mathrm{ND}$ & $\mathrm{ND}$ & $\mathrm{ND}$ \\
\hline MOPL & TSCA & WS code 1997 - MLLW & 0.12 & 0 & ND & $\mathrm{ND}$ & $\mathrm{ND}$ \\
\hline REME & $\mathrm{TSCA}$ & WS code 2006 - MLLW & 0.68 & 0 & ND & ND & ND \\
\hline \multirow[t]{2}{*}{ REME } & TSCA & WS code 2406 - MLLW & 5.32 & 0 & ND & $\mathrm{ND}$ & ND \\
\hline & & TSCA TOTALS: & 60,225 & $119,574,549$ & & $3,063,320$ & \\
\hline
\end{tabular}




\begin{tabular}{|c|c|c|c|c|c|c|c|c|c|c|c|c|c|}
\hline \multirow[b]{2}{*}{$\begin{array}{c}\text { Cons max } \\
\text { DRF }\end{array}$} & \multicolumn{3}{|c|}{$\mathrm{Hg}$} & \multicolumn{4}{|c|}{ PCBs } & \multicolumn{3}{|c|}{ Partial } & \multicolumn{3}{|c|}{ Max. upgrades } \\
\hline & $\begin{array}{c}\text { avg conc } \\
\text { (ppaw) }\end{array}$ & \begin{tabular}{|c} 
Est. mass \\
$($ (b)
\end{tabular} & Cons. DRF & $\begin{array}{c}\text { avg conc } \\
\text { (ppmw) }\end{array}$ & $\begin{array}{c}\text { Est. mass } \\
\text { (b) }\end{array}$ & DRF partial & DRF max. & $\begin{array}{c}\text { Max. } \\
\text { derating } \\
\text { factor }\end{array}$ & $\begin{array}{c}\text { Const. w/max } \\
\text { derating }\end{array}$ & $\begin{array}{c}\text { Ability to treat } \\
\text { stream }\end{array}$ & $\begin{array}{c}\text { Max. } \\
\text { derating } \\
\text { factor }\end{array}$ & $\begin{array}{l}\text { Const. } \\
\text { w/max } \\
\text { derating }\end{array}$ & $\begin{array}{c}\text { Ability to } \\
\text { treat stream }\end{array}$ \\
\hline 1 & 0 & 0.0 & 1 & & & & & 1 & & ok & 1 & & ok \\
\hline 1 & 0 & 0.0 & 1 & & & & & 1 & & ok & 1 & & ok \\
\hline 1 & 11.1 & 4.8 & 1 & & & & & 1 & & ok & 1 & & ok \\
\hline 1 & 11.1 & 36 & 1 & & & & & 1 & & ok & 1 & & ok \\
\hline 1 & 540 & 19 & 10 & & & & & 10 & $\mathrm{Hg}$ & ok & 10 & $\mathrm{Hg}$ & ok \\
\hline 1 & 0 & 0.0 & 1 & & & & & 1 & & ok & 1 & & ok \\
\hline 1 & 0.4 & 1.8 & 1 & & & & & 1 & & ok & 1 & & olk \\
\hline 1 & 11.1 & 301 & 1 & & & & & 1 & & ok & 1 & & ok \\
\hline 1 & 11.1 & 505 & 1 & & & & & 1 & & ok & 1 & & ok \\
\hline 1 & 11.1 & 1.4 & 1 & & & & & 1 & & ok & 1 & & ok \\
\hline & & 868 & & & & & & 1.004 & & $80,955,851$ & 1.004 & & $80,955,851$ \\
\hline 1.13 & 2.1 & 0.3 & 1 & 47,215 & 6,902 & 9,443 & 944 & 9,443 & PCBs & $x-P C B s$ & 944 & PCBs & ok \\
\hline 5.71 & 17 & 1.5 & 1 & 212,544 & 19.213 & 42,509 & 4,251 & 42.509 & PCBs & $x$-PCBs,Cl & 4,251 & PCBs & ok \\
\hline 5.71 & 17 & 1.2 & 1 & 212.544 & 15,068 & 42.509 & 4.251 & 42,509 & PCBs & $x-\mathrm{PCBs}, \mathrm{CI}$ & 4,251 & PCBs & ok \\
\hline 1 & 0.8 & 0.2 & 1 & 10,830 & 2,279 & 2.166 & 217 & 2,166 & PCBs & x-PCBs & 217 & PCBs & ok \\
\hline 3.57 & 14 & 8.9 & 1 & 122,422 & 79,279 & 24,484 & 2,448 & 24,484 & PCBs & x-PCBs & 2,448 & PCBs & ok \\
\hline ND & ND & $\mathrm{ND}$ & $\mathrm{ND}$ & $\mathrm{ND}$ & ND & $\mathrm{ND}$ & ND & ND & $?$ & $?$ & $\mathrm{ND}$ & $?$ & $?$ \\
\hline 1 & 0.5 & 0.1 & 1 & 843 & 140 & 169 & 17 & 169 & PCBs & $x-$ PCBs & 17 & PCBs & ok \\
\hline 2.28 & 7.3 & 1.8 & 1 & 86.680 & 21,124 & 17,336 & 1,734 & 17.336 & PCBs & $x-P C B s$ & 1.734 & PCBs & ok \\
\hline 2.28 & 7.3 & 29 & 1 & 86,680 & 340,194 & 17,336 & 1,734 & 17,336 & PCBs & $x-P C B s$ & 1,734 & PCBs & olk \\
\hline 1.55 & 36 & 40 & 1 & 224 & 247 & 45 & 4 & 45 & PCBs & ok & 4 & PCBs & ok \\
\hline 1 & 3.0 & 3.9 & 1 & 5.118 & 6.762 & 1 & 0.1 & 1 & PCBs & ok & 1 & & ok \\
\hline 1.13 & 2.1 & 6.5 & 1 & 47,215 & 147,944 & 9,443 & 944 & 9,443 & PCBs & x-PCBs & 944 & PCBs & ak \\
\hline 1.13 & 2.1 & 0.5 & 1 & 47,215 & 12.227 & 9,443 & 944 & 9,443 & PCBs & $x$-PCBs & 944 & PCBs & ok \\
\hline 1.13 & 2.1 & 0.0 & 1 & 47,215 & 1,003 & 9,443 & 944 & 9,443 & PCBs & $\mathrm{x}-\mathrm{PCBs}$ & 944 & PCBs & olk \\
\hline 1 & 0.6 & 0.0 & 1 & 11,833 & 391 & 2367 & 237 & 2.367 & PCBs & $\mathrm{X}-\mathrm{PCBS}$ & 237 & PCBs & ok \\
\hline 1.13 & 2.1 & 0.0 & 1 & 47,215 & 32.161 & 9,443 & 944 & 9,443 & PCBs & ok & 944 & PCBs & ok \\
\hline 1 & 0.8 & 0.0 & 1 & 9,439 & 96.567 & 1,888 & 189 & 1,888 & PCBs & $x$-PCBs & 189 & PCBs & ok \\
\hline 1 & 0.6 & 0.0 & 1 & 12,819 & 68.540 & 2.564 & 256 & 2,564 & PCBs & $x$-PCBs & 256 & PCBs & ak \\
\hline 1 & 0.6 & 0.0 & 1 & 13,797 & 22.883 & 2.759 & 276 & 2.759 & PCBs & $\mathrm{x}-\mathrm{PCBs}$ & 276 & PCBs & ok \\
\hline 2.78 & 7.8 & 0.0 & 1 & 100,464 & 26.310 & 20,093 & 2,009 & 20,093 & PCBs & $x-P C B s$ & 2,009 & PCBs & ok \\
\hline $\mathrm{ND}$ & ND & $\mathrm{ND}$ & $\mathrm{ND}$ & $\mathrm{ND}$ & $\mathrm{ND}$ & $\mathrm{ND}$ & ND & ND & $?$ & $?$ & ND & $?$ & $?$ \\
\hline 2.21 & 9.1 & 0.1 & 1 & 74,984 & 871 & 14,997 & 1,500 & 14,997 & PCBs & $x-P C B s$ & 1,500 & PCBs & ok \\
\hline 1 & 0.8 & 83 & 1 & 10,830 & $1,171,308$ & 2,166 & 217 & 2,166 & PCBs & $x-P C B s$ & 217 & PCBS & ok \\
\hline 1 & 1.3 & 0.0 & 1 & 42,740 & 0.000 & 8.548 & 855 & 8.548 & PCBs & $x-P C B s$ & 855 & PCBs & ok \\
\hline 2.20 & 17 & 0.0 & 1 & 55.200 & 0.000 & 11,040 & 1,104 & 11,040 & PCBs & $x-P C B s$ & 1,104 & PCBs & ok \\
\hline 1 & 0.8 & 0.0 & 1 & 10,830 & 0.000 & 2.166 & 217 & 2.166 & PCBs & $x-P C B s$ & 217 & PCBs & ok \\
\hline 1 & 0.8 & 0.0 & 1 & 10,830 & 0.000 & 2,166 & 217 & 2,166 & PCBs & $x-\mathrm{PCBs}$ & 217 & PCBS & ok \\
\hline 1 & 0.8 & 0.0 & 1 & 10,830 & 0.000 & 2,166 & 217 & 2,166 & PCBs & $x-$ PCBs & 217 & PCBs & ok \\
\hline
\end{tabular}




\begin{tabular}{|c|c|c|c|c|c|c|c|c|c|c|c|c|c|}
\hline \multirow[b]{2}{*}{$\begin{array}{c}\text { Cons max } \\
\text { DRF }\end{array}$} & \multicolumn{3}{|c|}{$\mathrm{Hg}$} & \multicolumn{4}{|c|}{ PCBs } & \multicolumn{3}{|c|}{ Partial } & \multicolumn{3}{|c|}{ Max upgrades } \\
\hline & $\begin{array}{l}\text { 2vg conc } \\
\text { (ppmw) }\end{array}$ & $\begin{array}{c}\begin{array}{c}\text { Est. mass } \\
\text { (lb) }\end{array} \\
\end{array}$ & Cons. DRF & $\begin{array}{l}\text { avg conc } \\
\text { (ppmw) }\end{array}$ & $\begin{array}{c}\text { Est. mass } \\
\text { (lb) }\end{array}$ & DRF partial & DRF max. & $\begin{array}{c}\text { Max. } \\
\text { derating } \\
\text { factor } \\
\end{array}$ & $\begin{array}{c}\text { Const. w/max } \\
\text { derating }\end{array}$ & $\begin{array}{c}\text { Ability to treat } \\
\text { stream }\end{array}$ & $\begin{array}{l}\text { Max. } \\
\text { derating } \\
\text { factor }\end{array}$ & $\begin{array}{c}\text { Const } \\
\text { w/max } \\
\text { derating }\end{array}$ & $\begin{array}{l}\text { Ability to } \\
\text { treat stream }\end{array}$ \\
\hline 1 & 1.3 & 0.0 & 1 & 42,740 & 0.000 & 8,548 & 855 & 8,548 & PCBs & $x$-PCBs & 855 & PCBs & ok \\
\hline 1 & 0.6 & 0.0 & 1 & 13,797 & 0.000 & 2,759 & 276 & 2759 & PCBs & $x-P C B s$ & 276 & PCBs & ok \\
\hline 2.20 & 17 & 0.0 & 1 & 55,200 & 0.000 & 11.040 & 1,104 & 11.040 & PCBs & $x$-PCBs & 1,104 & PCBs & ok \\
\hline 2.78 & 7.8 & 0.0 & 1 & 100,464 & 0.000 & 20,093 & 2.009 & 20,093 & PCBs & $\mathrm{x}$-PCBs & 2,009 & PCBs & ok \\
\hline 1 & 0.6 & 0.0 & 1 & 12,819 & 0.000 & 2,564 & 256 & 2,564 & PCBs & x-PCBs & 256 & PCBs & ok \\
\hline 1 & 0.5 & 0.0 & 1 & 843 & 0.000 & 169 & 17 & 169 & PCBs & $x-P C B s$ & 17 & PCBs & ok \\
\hline 1 & 0.9 & 0.0 & 1 & 11.793 & 0.000 & 2,359 & 236 & 2,359 & PCBs & X-PCBs & 236 & PCBs & ok \\
\hline 1.13 & 2.1 & 0.0 & 1 & 47,215 & 0.000 & 9.443 & 944 & 9,443 & PCBs & $x-P C B s$ & 944 & PCBs & ok \\
\hline 1.13 & 2.1 & 0.0 & 1 & 47.215 & 0.000 & 9.443 & 944 & 9,443 & PCBs & $x-P C B_{s}$ & 944 & PCBs & ok \\
\hline ND & ND & $\mathrm{ND}$ & $\mathrm{ND}$ & $\mathrm{ND}$ & $\mathrm{ND}$ & ND & ND & $\mathrm{ND}$ & $?$ & $?$ & ND & $?$ & $?$ \\
\hline $\mathrm{ND}$ & $\mathrm{ND}$ & ND & ND & ND & $\mathrm{ND}$ & ND & $\mathrm{ND}$ & $\mathrm{ND}$ & $?$ & $?$ & ND & $?$ & $?$ \\
\hline ND & $\mathrm{ND}$ & $\mathrm{ND}$ & $\mathrm{ND}$ & ND & $\mathrm{ND}$ & ND & ND & ND & $?$ & $?$ & $\mathrm{ND}$ & $?$ & $?$ \\
\hline $\mathrm{ND}$ & $\mathrm{ND}$ & $\mathrm{ND}$ & ND & $\mathrm{ND}$ & $\mathrm{ND}$ & ND & ND & $\mathrm{ND}$ & $?$ & $?$ & $\mathrm{ND}$ & $?$ & $?$ \\
\hline \multirow[t]{2}{*}{ ND } & $\mathrm{ND}$ & ND & ND & ND & ND & ND & ND & ND & $?$ & $?$ & ND & $?$ & $?$ \\
\hline & & 176 & & & $1,818,443$ & & & 3,042 & & $2,426,983$ & 304.16 & & $119.555,341$ \\
\hline
\end{tabular}


Table A-8. (continued).

\begin{tabular}{|c|c|c|c|c|c|c|c|c|}
\hline & & & & & \multicolumn{4}{|c|}{$\mathrm{Cl}$} \\
\hline Generator & $\begin{array}{l}\text { Planned } \\
\text { receiver }\end{array}$ & Waste Description & Vol. $\left(\mathrm{m}^{3}\right)$ & $\begin{array}{c}\text { Est. mass } \\
\text { (b) }\end{array}$ & $\begin{array}{l}\text { avg conc } \\
\text { (ppmw) }\end{array}$ & $\begin{array}{c}\text { Est. mass } \\
\text { (lb) }\end{array}$ & $\begin{array}{c}\text { Cons. DRF } \\
\text { partial }\end{array}$ & \\
\hline PORTS. & TSCA & TSCA liquids & $1,914.4$ & 0 & 43,886 & 0.000 & 1.76 & 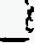 \\
\hline PORTS. & TSCA & MW incinerable liquids & 539 & lo & 22,143 & 0.000 & 1 & \\
\hline PORTS. & TSCA & TSCA soft solids & 1.871 & 0 & 109.956 & 0.000 & 4.40 & : \\
\hline PORTS. & TSCA & Sorted MW incin. soft solids & 4,437 & 0 & 138,880 & 0.000 & 5.56 & . \\
\hline RF & TSCA & PCB organic liquids & 127 & 0 & 24,435 & 0.000 & 1 & : \\
\hline RF & TSCA & PCB organic solids & 17 & 0 & 8,337 & 0.000 & 1 & - \\
\hline SNL & TSCA & Septage liquids & 15 & 0 & 12110 & 0.000 & 1 & \\
\hline SRS & TSCA & Incinerable rad. PCB & 1 & 0 & 56,357 & 0.000 & 2.25 & \\
\hline W.VALIEY & TSCA & PCB wastes liguids/solids & 6.63 & 10 & 56,357 & 0.000 & 2.25 & \\
\hline BCLU & TSCA & IWS code 1987-MLLW & 2.45 & 0 & ND & ND & ND & \\
\hline MOPL & TSCA & WS code 1996 - MLLW & 0.76 & 0 & ND & $\mathrm{ND}$ & ND & - \\
\hline MOPL & TSCA & WS code 1997 - MLLW & 0.12 & 0 & ND & ND & ND & \\
\hline REME & ISCA & WS code 2006 - MLLW & 0.68 & 0 & ND & ND & ND & $\therefore$ \\
\hline REME & TSCA & WS code 2406 - MLLW & 5.32 & 0 & $\mathrm{ND}$ & ND & ND & \\
\hline & & ISCA TOTALS: & 60.225 & 119.574 .549 & & $3,063,320$ & & \\
\hline
\end{tabular}




\section{Appendix B \\ Material and Energy Balance Calculations}




\section{Appendix B}

\section{Material and Energy Balance Calculations}

Material and energy balances were calculated for the four proposed equipment configurations under evaluation. These calculations were performed to enable the task study team to understand the process variables that significantly influence the Air Pollution Control (APC) system design, cost and complexity.

A process simulation code, APEN PLUS (ASPEN), was used to calculate material and energy

Balances for each unit operation in the WERF incineration and APC systems. An ASPEN simulation flowsheet consists of blocks and streams. Each block representing a unit operation must have its operating conditions and performance specified, or ASPEN can calculate the operating conditions and performance necessary to achieve given or desired process conditions or stream conditions. Material streams enter and exit blocks. Initial feedstreams must have their stream conditions and composition specified by the user. Intermediate streams between blocks and final product streams are the results of material and energy calculations within the block.

The user must also specify the components in the process and the physical property databanks to be used. ASPEN contains an extensive database to calculate physical and thermodynamic properties of gases, vapors, liquids, inorganic compounds, and hydrocarbons. If physical property parameters do not exist in ASPEN for a component, the user can add those properties to the database so that the missing physical properties can be estimated.

A description of the process and operating conditions, as well as assumptions made in calculating the material and energy balance equations around each unit operation in the proposed APCS, are listed below.

\section{B-1. Controlled-air Incinerator}

The WERF incinerator is a dual-chamber controlled air incinerator. The waste is fed into the first, or PCC in a controlled-air, or oxygen-starved environment designed to minimize particulate carryover. The PCC is typically operated at temperatures between $1600-2000^{\circ} \mathrm{F}{ }^{\mathrm{A}-1}$ Additional air is added between the primary and secondary combustion chambers. In the SCC, incomplete combustion products are fully combusted at temperatures between $1800-2000^{\circ} \mathrm{F}$. The dual-chamber incinerator was modeled as a single unit operation with an exit gas temperature of $1900^{\circ} \mathrm{F}$. A heat loss of 0.5 $\mathrm{Mbtu} / \mathrm{hr}$ from the incinerator was assumed. This is close to the $7 \%$ loss that was assumed in other material and energy balance equations. ${ }^{A-2}$

The waste feed rate into the incinerator was specified to be $400 \mathrm{lbs} / \mathrm{hr}$. This value is the result of a feed capacity limit of $5 \mathrm{Mbtu} / \mathrm{hr}$ and the assumption that the waste has a heating value of 12,500 $\mathrm{Btu} / \mathrm{lb} .^{\mathrm{A}-1}$ A fuel oil feed rate of $75 \mathrm{lbs} / \mathrm{hr}$ (heating value of approximately $20,000 \mathrm{Btu} / \mathrm{lb}$ ) was assumed; this resulted in a total release of approximately $6.5 \mathrm{Mbtru} / \mathrm{hr}$, which is the thermal capacity of the incinerator. ${ }^{A-1}$ The waste feed stream consisted of sufficient polyvinyl chloride (PVC) to ensure a $20 \mathrm{lbs} / \mathrm{hr}$ chlorine feed rate with the difference being polyethylene. The cellulose material consisted of packaging materials (cardboard and shredded comcobs). The corncob filler is assumed to compose $84.3 \%$ of total cellulose mass in the waste. The assumed composition and heating values of the polymers, cellulose material, and fuel oil are listed in Table A-1. 
Table B-1. Assumed compositional analysis of organic material incinerated at WERF.

\begin{tabular}{lllll}
\hline Constituents (Mass \%) & Cardboard & Corncob Filler & Polymers & Fuel Oil \\
\hline Volatiles & 77 & 73 & - & 99.5 \\
Fixed carbon & 12 & 17.8 & - & - \\
Water & 5 & 7 & -3 & - \\
Ash & 6 & - & - & - \\
Sulfur & - & - & 123.64 & 0.5 \\
Polyethylene & - & - & 36.36 & - \\
Polyvinyl Chloride & - & 8000 & 20000 & - \\
Heating Value (Btu/lb) & 8000 & & & 19,500 \\
\hline
\end{tabular}

The resulting heating value for this mixture was calculated to be $12,800 \mathrm{Btu} / \mathrm{lb}$. In addition, the design waste feed stream contains mercury $(\mathrm{Hg})$ at concentrations sufficient to be fed at a rate of $0.022 \mathrm{lb} / \mathrm{hr}(10 \mathrm{~g} / \mathrm{hr})$. The amount of air added between the PCC and SCC was in excess of that needed for complete combustion of the available organic material. In the ASPEN model, the amount of air added was that required to cool the exiting offgas to $1900^{\circ} \mathrm{F}$.

The four APC systems described in Section 4 were modeled using the waste feed stream as described above. Each APCS option with its new major components is listed in Table A-2. Basically, the significant difference between each option is the method used to remove the $\mathrm{HCl}$ from the offgas. The types of $\mathrm{Hg}$ and dioxin/furan control equipment are the same for all four cases. However, their sizes can vary depending upon the volumetric flow of the offgas requiring treatment.

\section{B-2. Evaporative Cooler}

The evaporative cooler is designed to rapidly cool the offgas from the SCC to minimize the formation of dioxins and furans. Spray nozzles ensure adequate distribution of water droplets of sufficiently small diameter to cool the offgas without droplet carryover out of the cooler. The evaporative cooler is designed to cool the offgas from $1900^{\circ} \mathrm{F}$ to $350^{\circ} \mathrm{F}$ assuming a heat loss of $0.25 \mathrm{Mbtu} / \mathrm{hr}$. A full-quench evaporative cooler is designed to cool offgas to a temperature where the offgas is fully saturated, which is around $165^{\circ} \mathrm{F}$.

\section{B-3. Chlorine Capture Processes}

Chlorine present in the waste is generally converted to hydrochloric acid $(\mathrm{HCl})$ with significantly smaller quantities present in the offgas as elemental chlorine or metal chlorides. Thermodynamic equilibrium calculations demonstrate that the formation of $\mathrm{HCl}$ is strongly preferred to that of chlorine. Metal chlorides form fine particulates upon cooling which are captured in a baghouse filter or high-efficiency particulate air (HEPA) filter. It was assumed that all chlorine fed into the incinerator is converted to $\mathrm{HCl}$. Three processes for removing $\mathrm{HCl}$ from the offgas were considered and the assumptions used in calculating the material and balances equations are summarized below.

Dry Sorbent Injection-A dry sorbent with the capability to absorb $\mathrm{HCl}$ injected upstream of the baghouse provides a means of capturing $\mathrm{HCl}$ without preparing or generating a liquid stream. Dry sorbents, such as hydrated lime and sodium bicarbonate, remove $\mathrm{HCl}$ when the offgas comes in contact with the solid additive. Most of the $\mathrm{HCl}$ removal is believed to occur in the baghouse where 
Table B-2. Proposed air pollution control systems for WERF.

Key APC Feature

APCS Components

Dry Sorbent Inheciton (DSI)

Spray dry Adsorption (SDA)

Wet Scrubber Upstream of Filtration

Wet Scrubber Downstream of Particulate

Removal
Evaporation cooler, DIS, carbon bed

Evaporative cooler, SDA, carbon bed

Evaporative cooler, full quench, wet scrubber, carbon bed

Evaporative cooler, full quench, wet scrubber, wet ESP, carbon bed

dry sorbent accumulates on the bag filter as a cake. The minimum stoichiometric amount of sorbent is generally insufficient to achieve complete $\mathrm{HCl}$ removal because of mass transfer limitations. Generally, dry sorbent is added at a 2:1 or higher ratio to achieve high removal efficiencies (95-98\%). The material balance assumed that hydrated lime $\left[\mathrm{Ca}(\mathrm{OH})_{2}\right]$ was fed in a stoichiometric ratio of 2:1 relative to $\mathrm{HCl}$ to achieve $97.2 \% \mathrm{HCl}$ removal. A heat loss of $0.1 \mathrm{MBtu} / \mathrm{hr}$ from the baghouse was assumed.

Spray Dry Absorption - A semi-dry process in which a liquid spray containing a material that reacts with the $\mathrm{HCl}$ and converts the chlorine to a solid compound was also considered for the WERF incinerator. In a spray dryer absorber, a liquid-containing caustic $(\mathrm{NaOH})$ is sprayed concurrently into a hot, offgas stream $\left(750^{\circ} \mathrm{F}\right)$ where the water evaporates leaving small reactive particulate with a large total surface area that absorbs and reacts with $\mathrm{HCl}$ in the offgas. Generally, $\mathrm{NaOH}$ is far more reactive with $\mathrm{HCl}$ than other sorbents; thus, far less excess material needs to be added into the process to achieve similar removal efficiencies. A molar stoichiometric ratio for $\mathrm{NaOH}: \mathrm{HCl}$ of 1.1:1 was assumed to achieve a removal efficiency of $97.2 \%$. The heat loss from the SDA process is assumed to be $0.1 \mathrm{MBtu} / \mathrm{hr}$.

Wet Scrubber-A wet scrubber located downstream of the baghouse will efficiently remove $\mathrm{HCl}$ and chlorine in the offgas. The offgas is saturated in another quench tower before contacting an aqueous stream with a pH between 7 and 9 to achieve high $\mathrm{HCl}$ removal efficiency (99.9\%). The problem remains what to do with the liquid waste streams containing the chlorine-containing salts. In Option 3 using a wet scrubber downstream of the HEPA filters, a process design was proposed in which the liquid waste stream from the scrubber was recycled back to the evaporative cooler where the water is evaporated, and the remaining salts and particulates are captured in the baghouse and HEPA filters. It was assumed that the recycle stream could be added in the evaporative cooler without any decrease in spray nozzle performance.

In Option 4 where a wet scrubber is located upstream of the particulate removal process, a venturi wet scrubber is used to capture acid gases and particulate. The liquid from the process is sent to an evaporator to concentrate the solids in solution and recycle the vapor condensate.

\section{B-4. Mercury Absorption by an Activated Carbon Bed}

Mercury $(\mathrm{Hg})$ volatized in an incinerator generally will not be removed from the offgas simply by cooling the offgas. It is necessary to actively remove $\mathrm{Hg}$ when present in large concentrations. Oxidized $\mathrm{Hg}$, such as $\mathrm{HgO}$ and $\mathrm{HgCl}_{2}$, exhibit high solubility in water and can be removed in a wet scrubber. Elemental $\mathrm{Hg}$ is not readily soluble in water with low $\mathrm{pH}$. One method identified as capable of removing elemental and oxidized forms of $\mathrm{Hg}$ was the use of a sulfur-impregnated activated carbon. The mercury reacts with the sulfur to form nonvolatile mercury sulfides that 
prevent $\mathrm{Hg}$ desorption as in the case of untreated activated carbon. The sulfur increases the total $\mathrm{Hg}$-loading capacity of the carbon. It was assumed that the carbon bed removes $99.4 \%$ of the $\mathrm{Hg}$ in the offgas. When necessary, air is added to the offgas after the baghouse to cool the offgas to $250^{\circ} \mathrm{F}$ before it enters the carbon bed. Sulfur-impregnated carbon from NUCON International is reported to operate at temperatures up to $390^{\circ} \mathrm{F}$ without loss of sulfur or mercury sulfide. ${ }^{\mathrm{A}-3}$ In the case of the wet scrubber, the scrubber offgas is heated to $250^{\circ} \mathrm{F}$ to prevent water condensation in the carbon bed.

\section{B-5. Air Pollution Control Systems}

The schematic of the APCS featuring dry sorbent injection and its process flowsheet identifying the pertinent streams and material flowrates are shown in Figure A-1 and Table A-3, respectively. The schematic of the APCS featuring spray dry absorption and its process flowsheet identifying streams and material flowrates are shown in Figure A-2 and Table A-4. The schematic of the APCS featuring a wet scrubber downstream of the baghouse and its process flowsheet identifying streams and material flowrates are shown in Figure A-3 and Table A.5. Figure A-4 and Table A-6 is the schematic and material flowrates for a wet scrubber located upstream of particulate removal.

The results indicate significantly lower total gas flowrates through the APCS as a result of eliminating several streams of air addition immediately after the SCC. Off gas cooling in an evaporative cooler results in significantly less offgas flows through the filters and out the stack. In fact, the volumetric offgas flowrate into the baghouse for all APC systems is between 7,000 and 8,000 acfm which is about $1 / 3$ to $1 / 2$ of the total gas now passing through the baghouse under current WERF operations. 


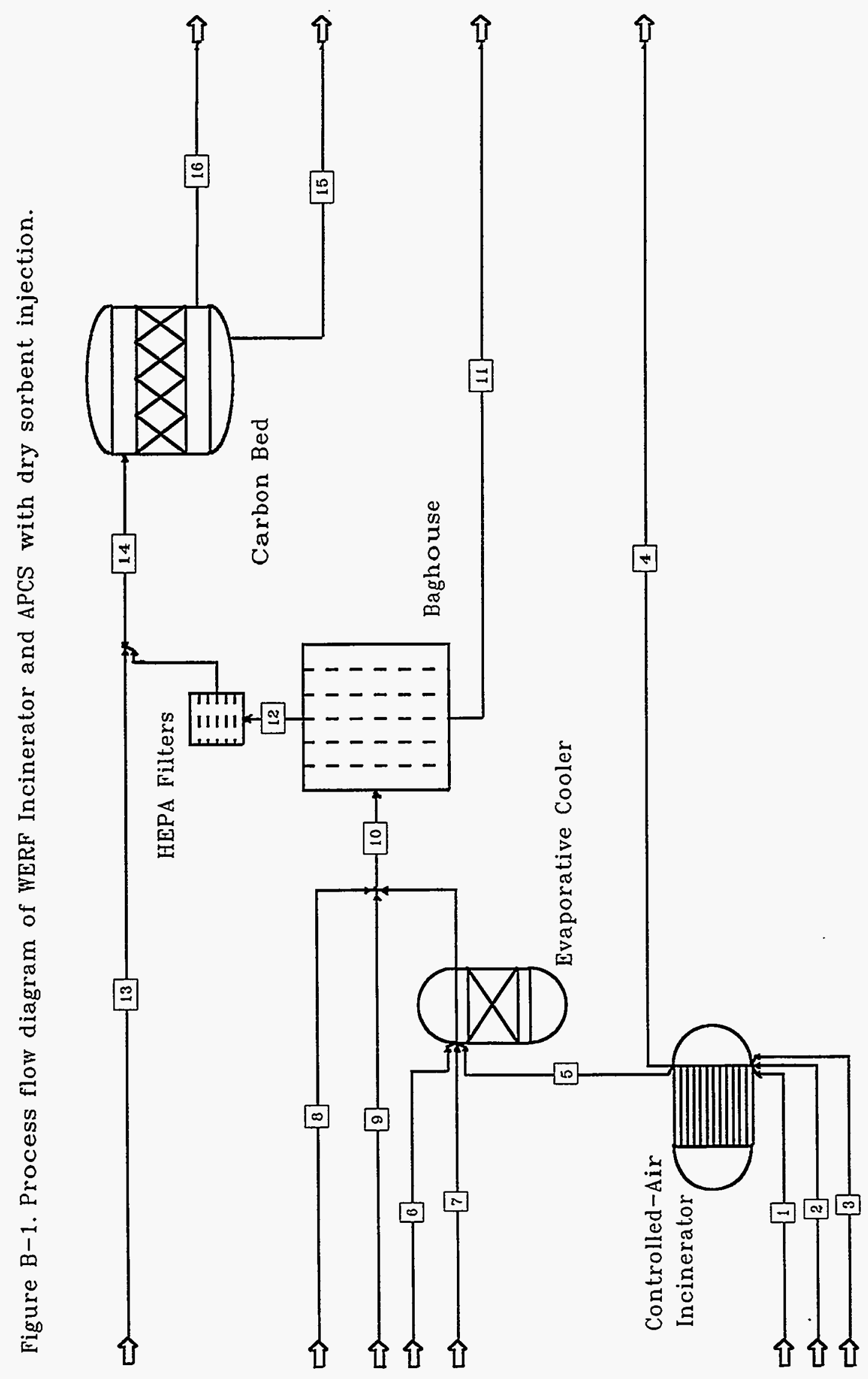




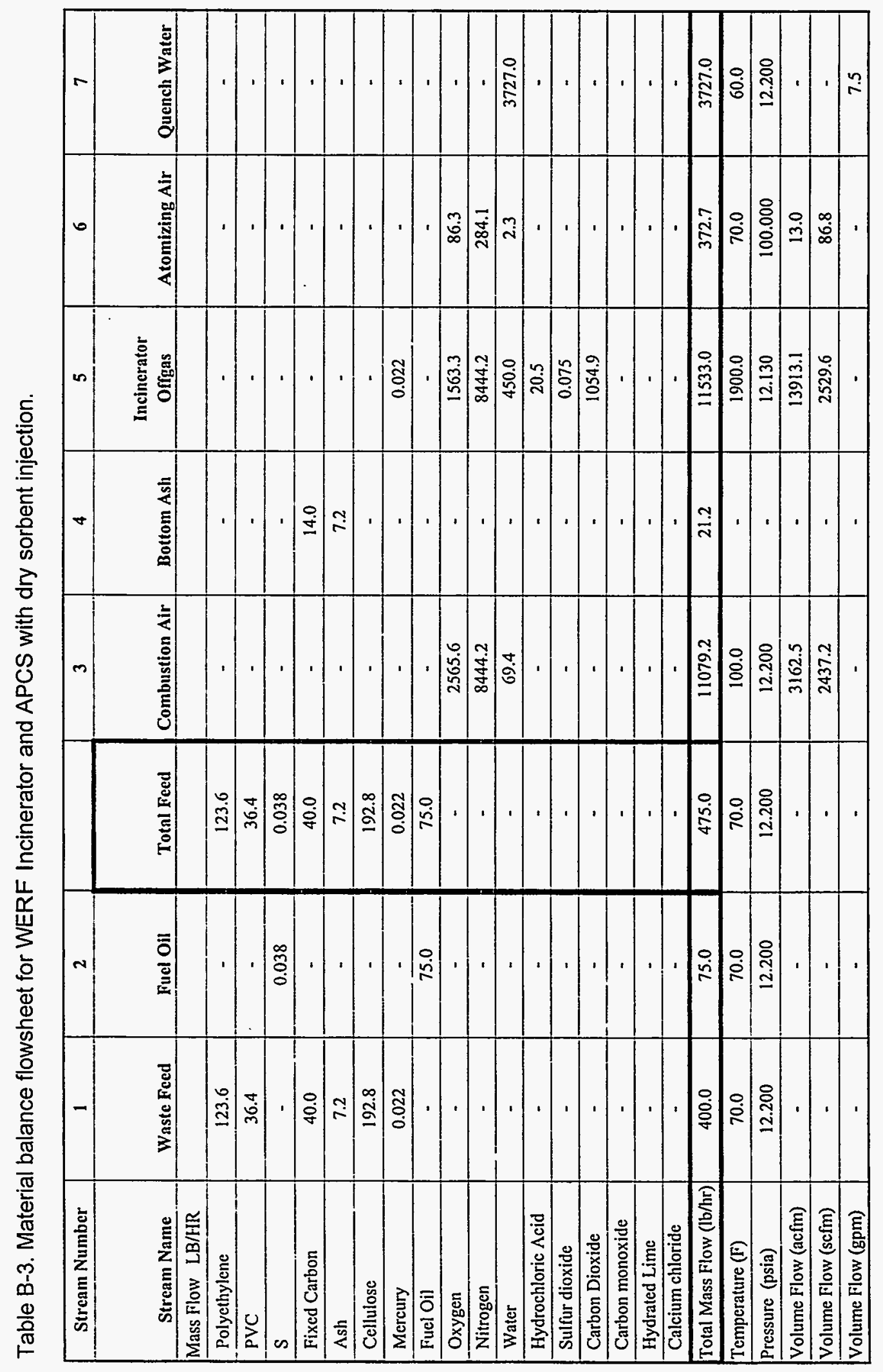




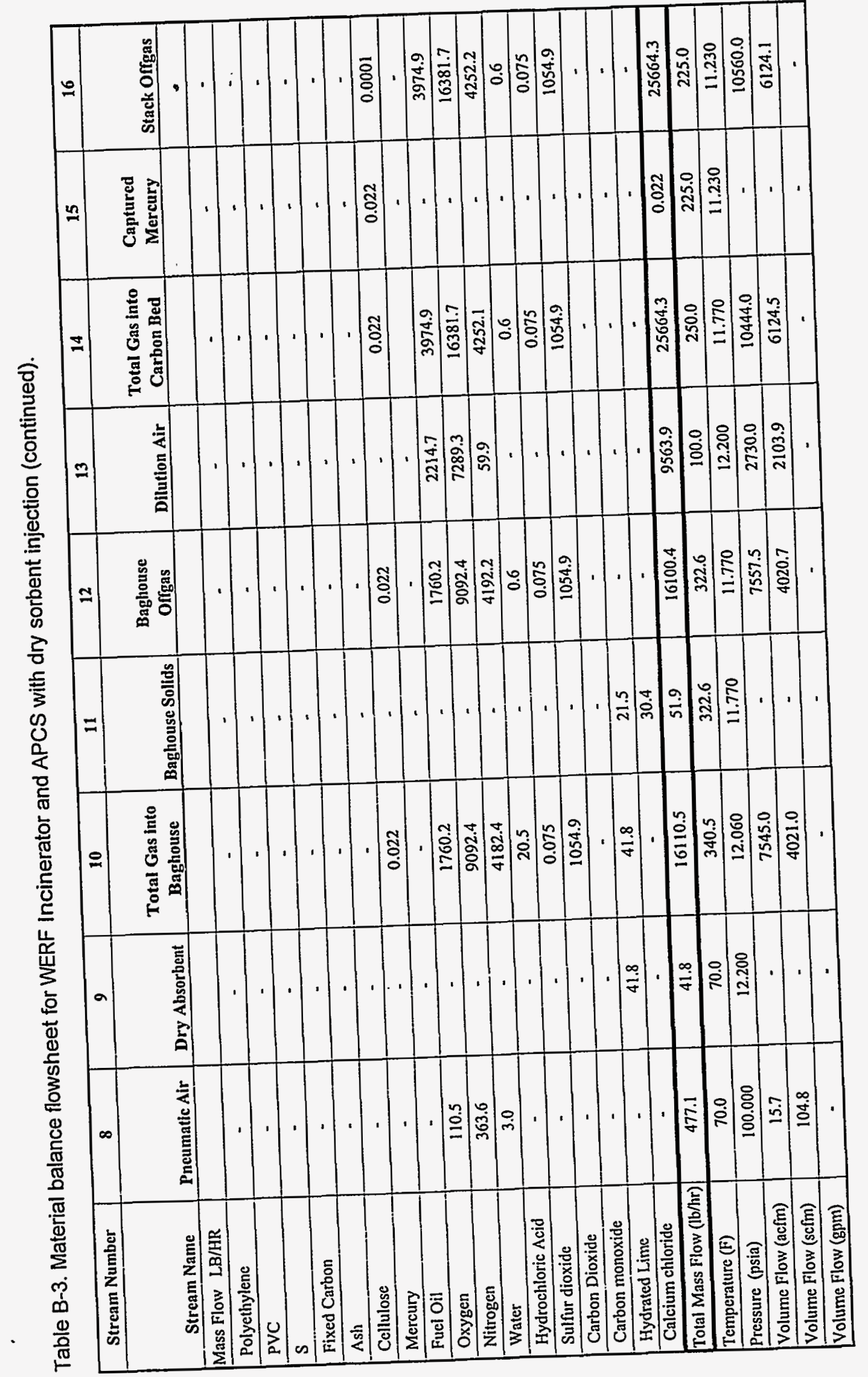




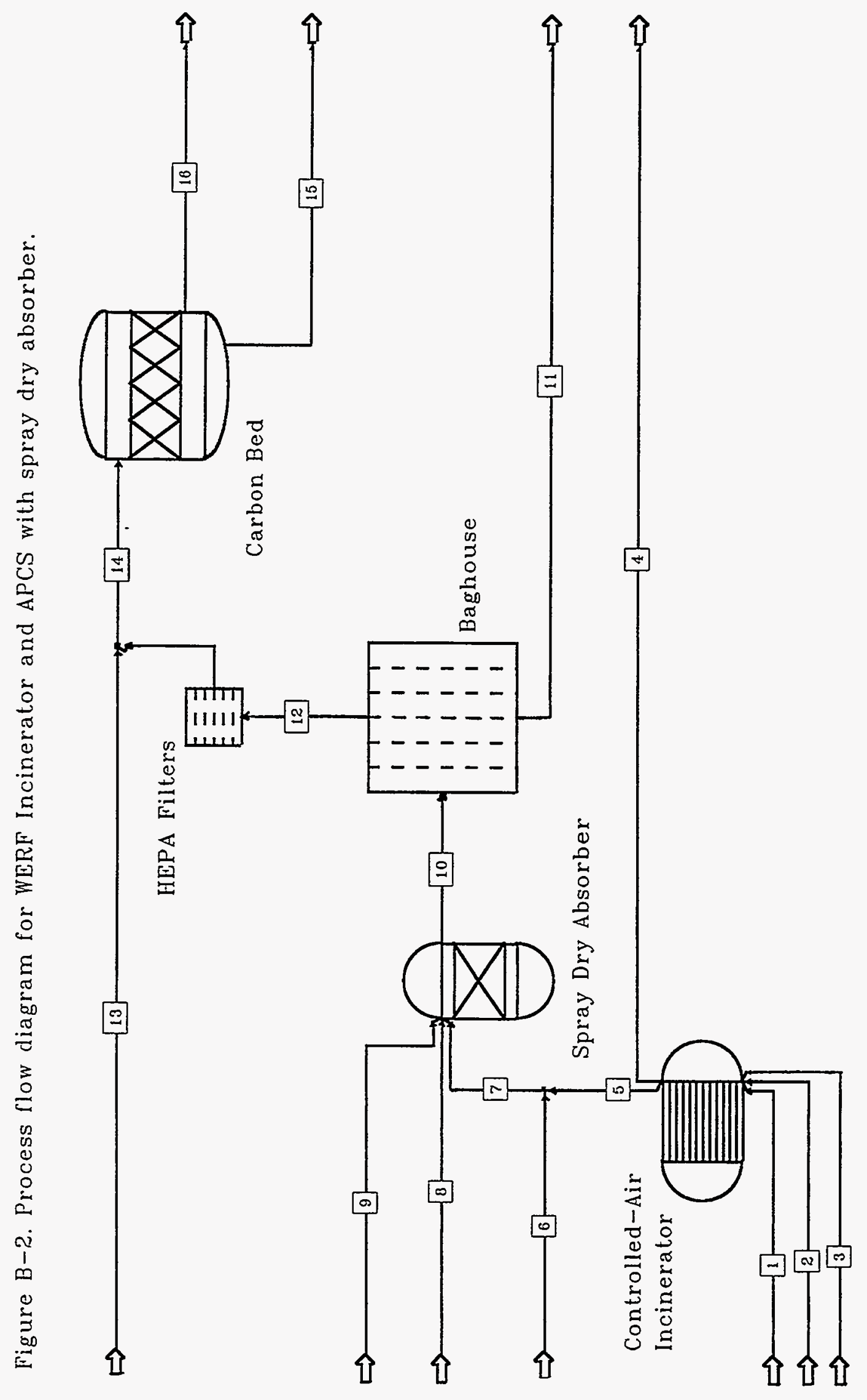




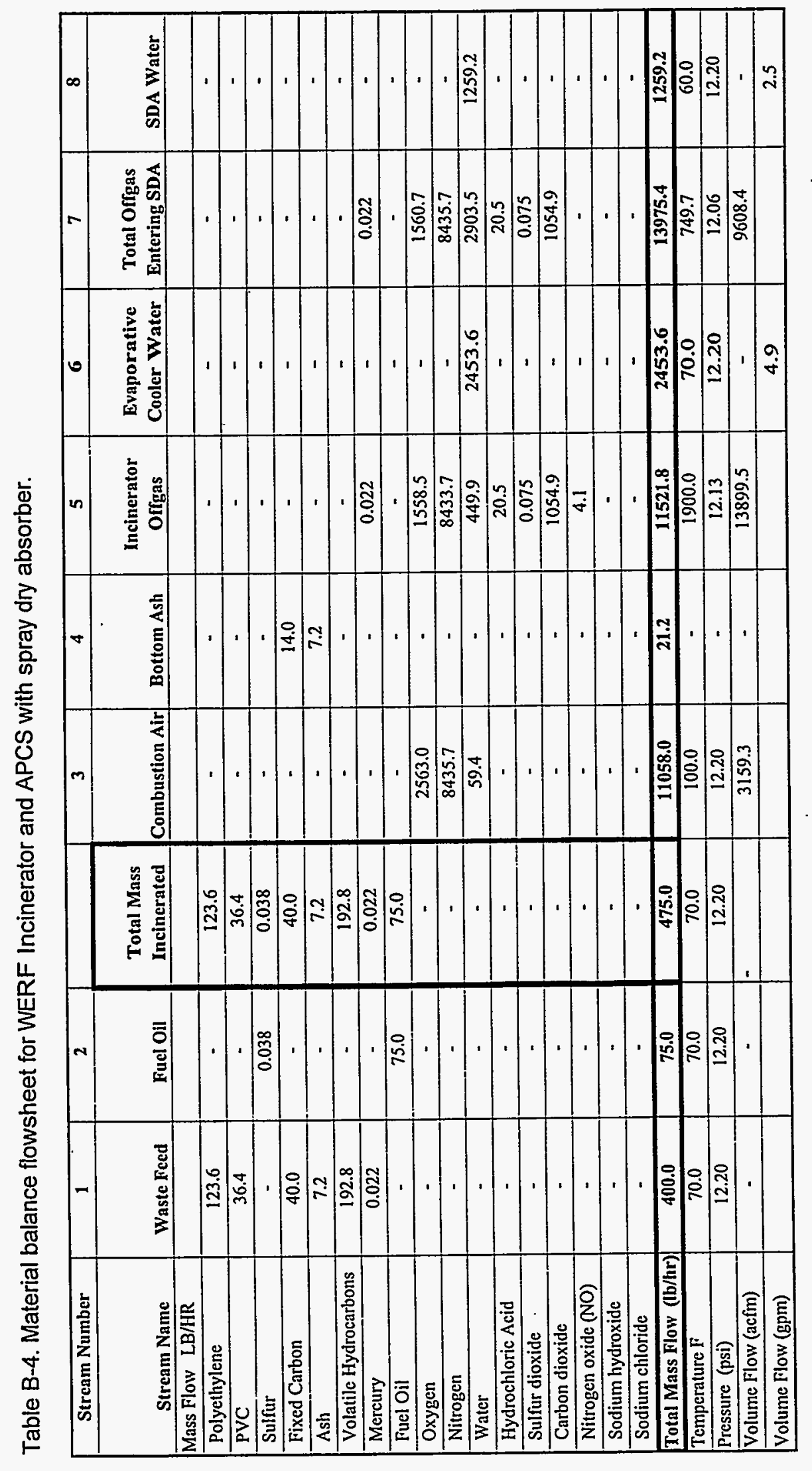




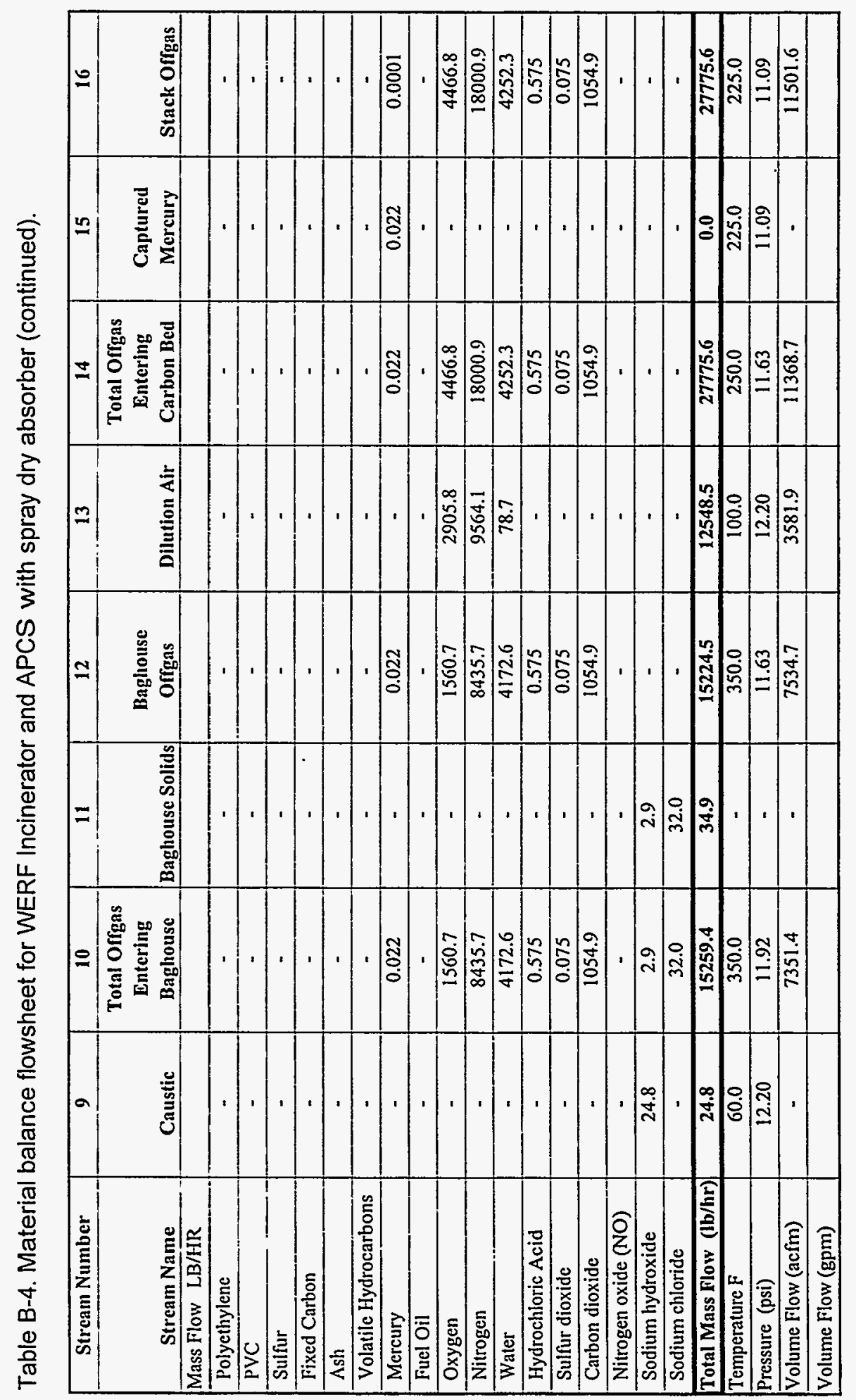




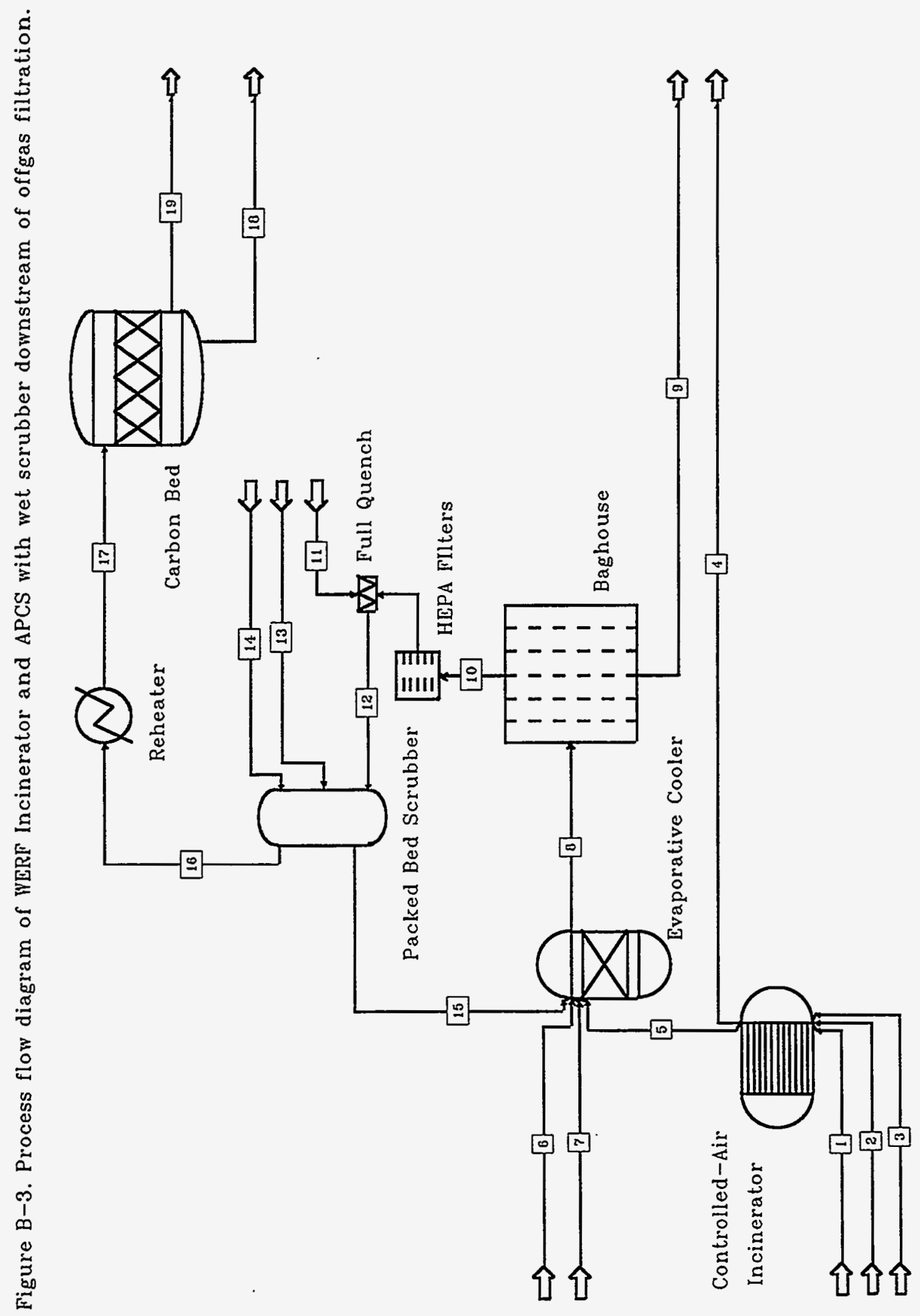




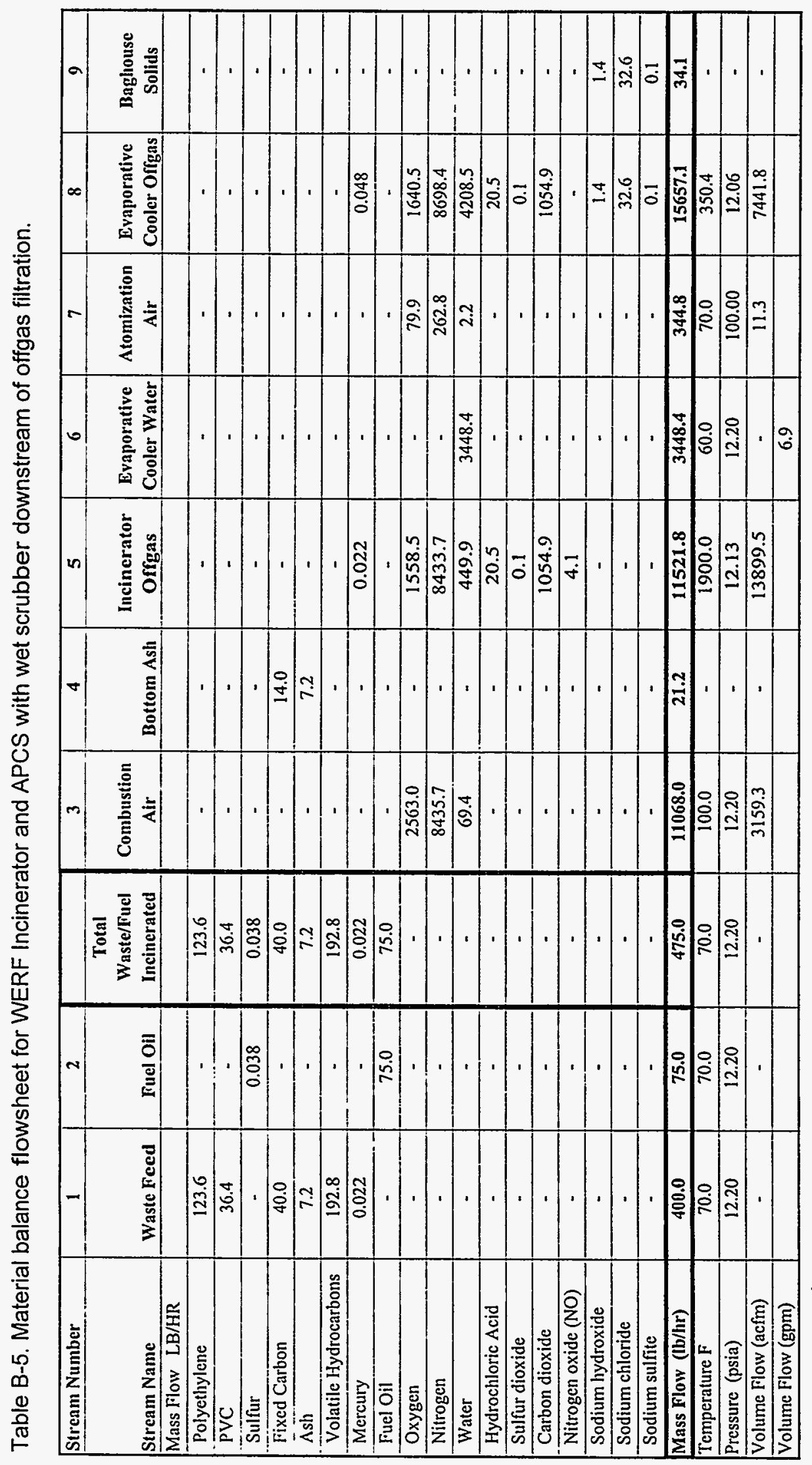




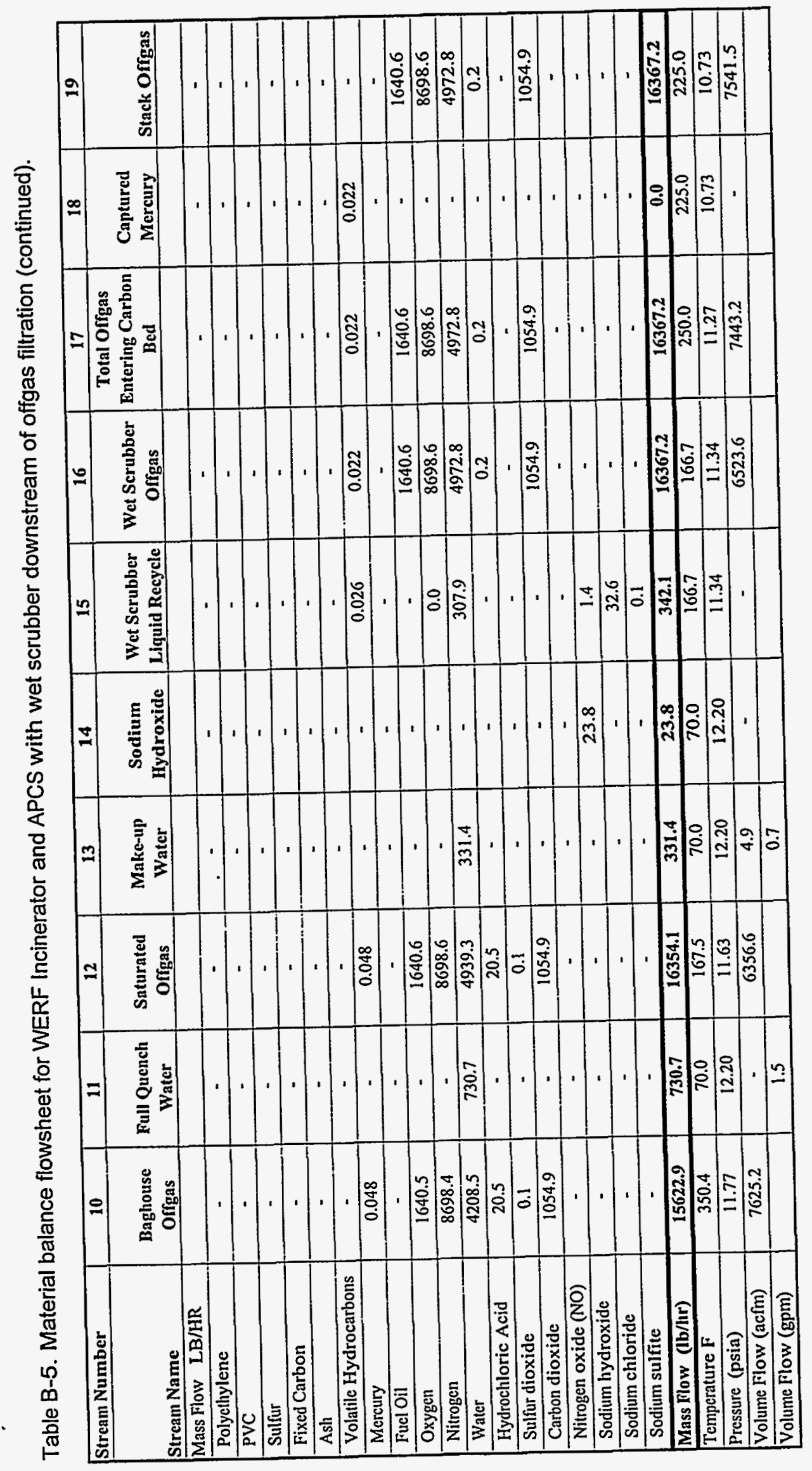




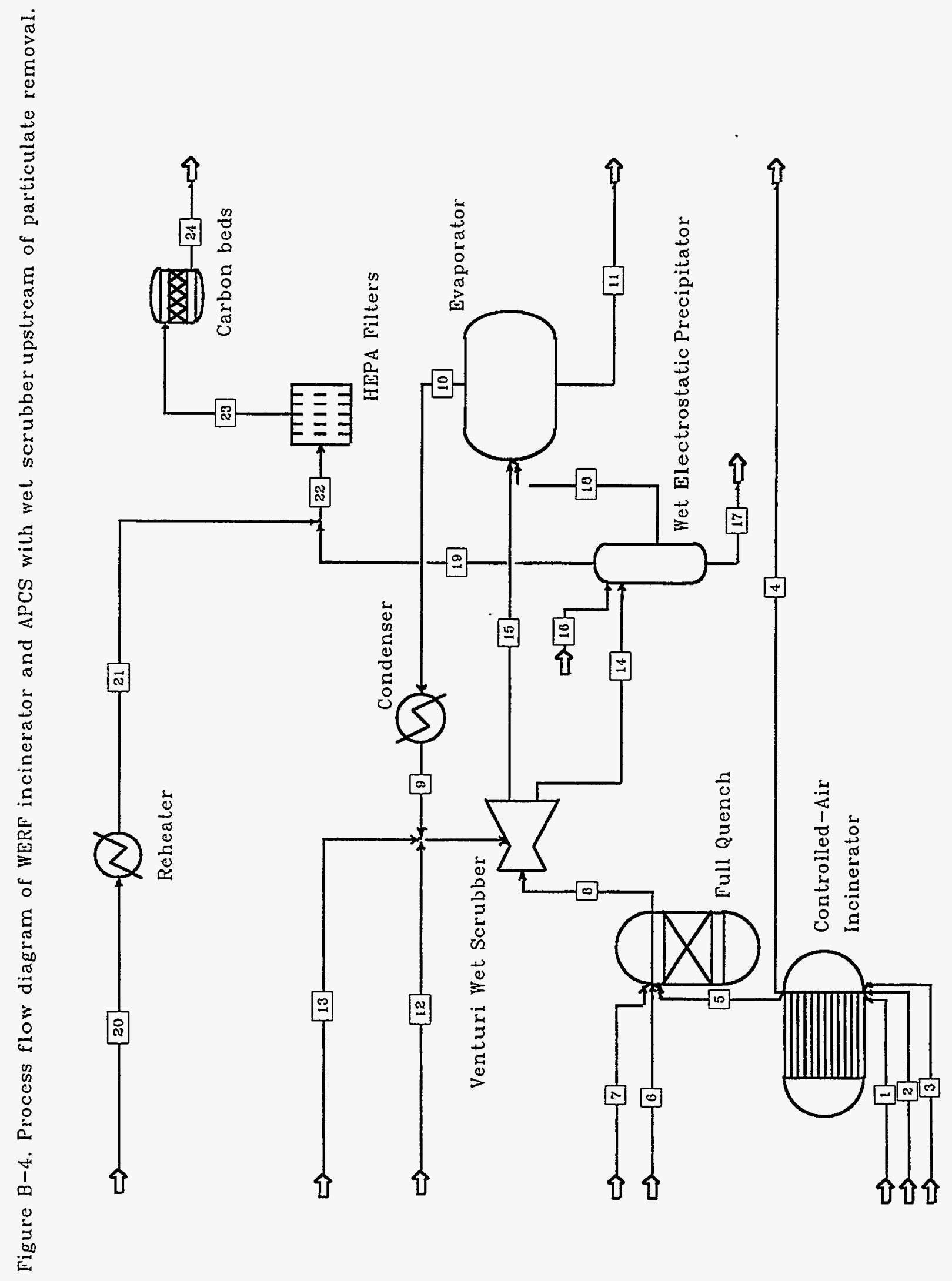




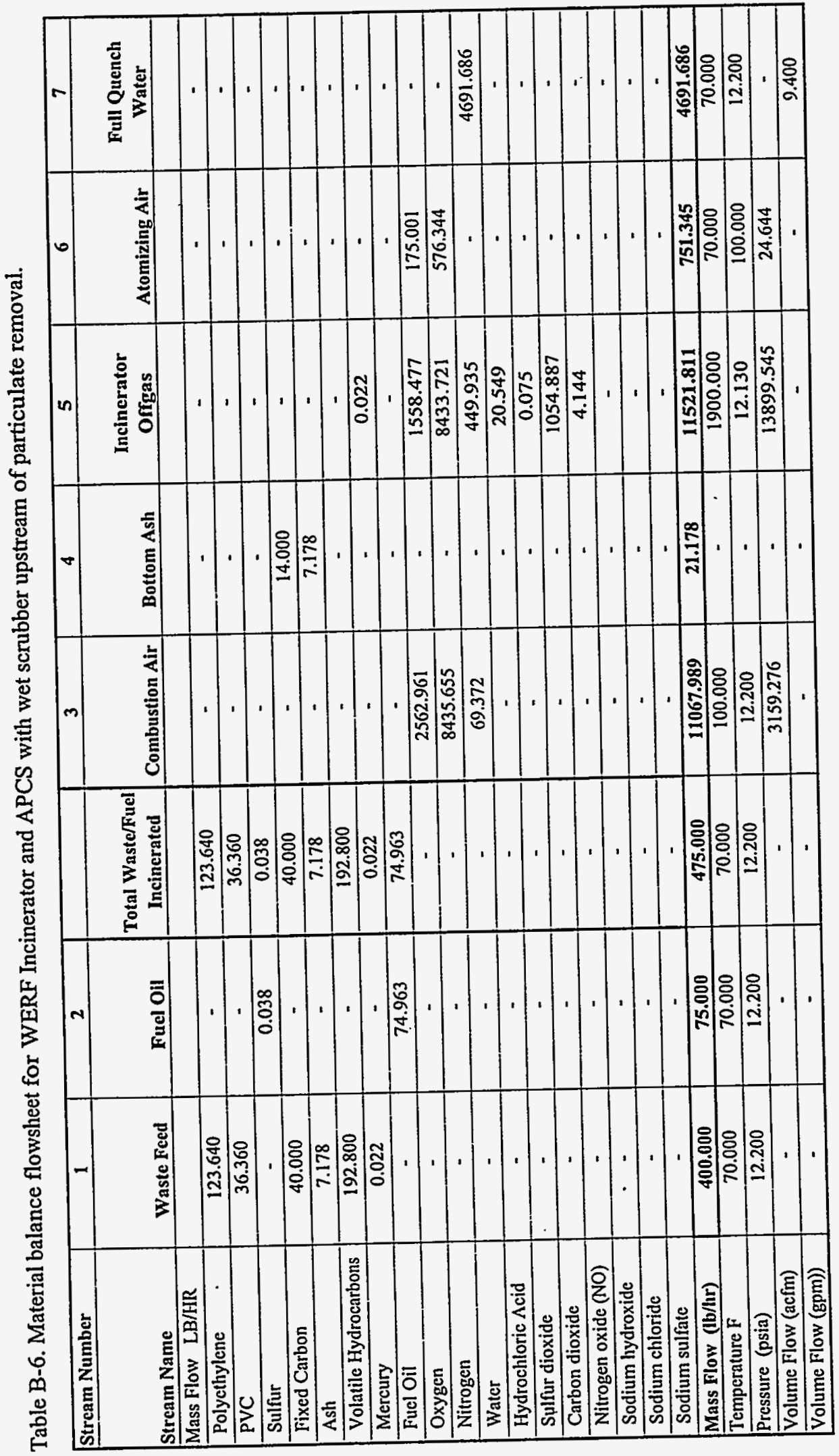




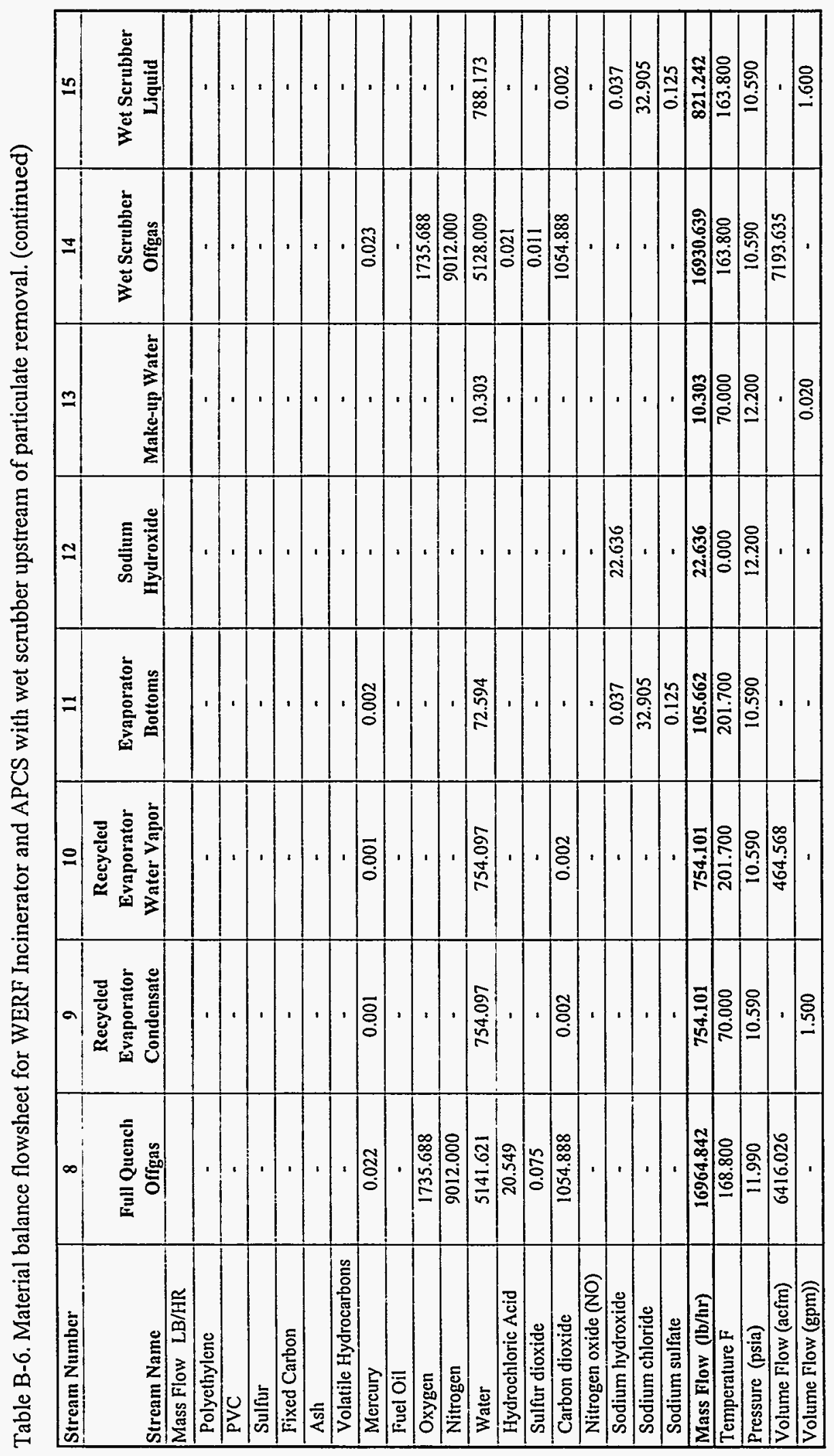




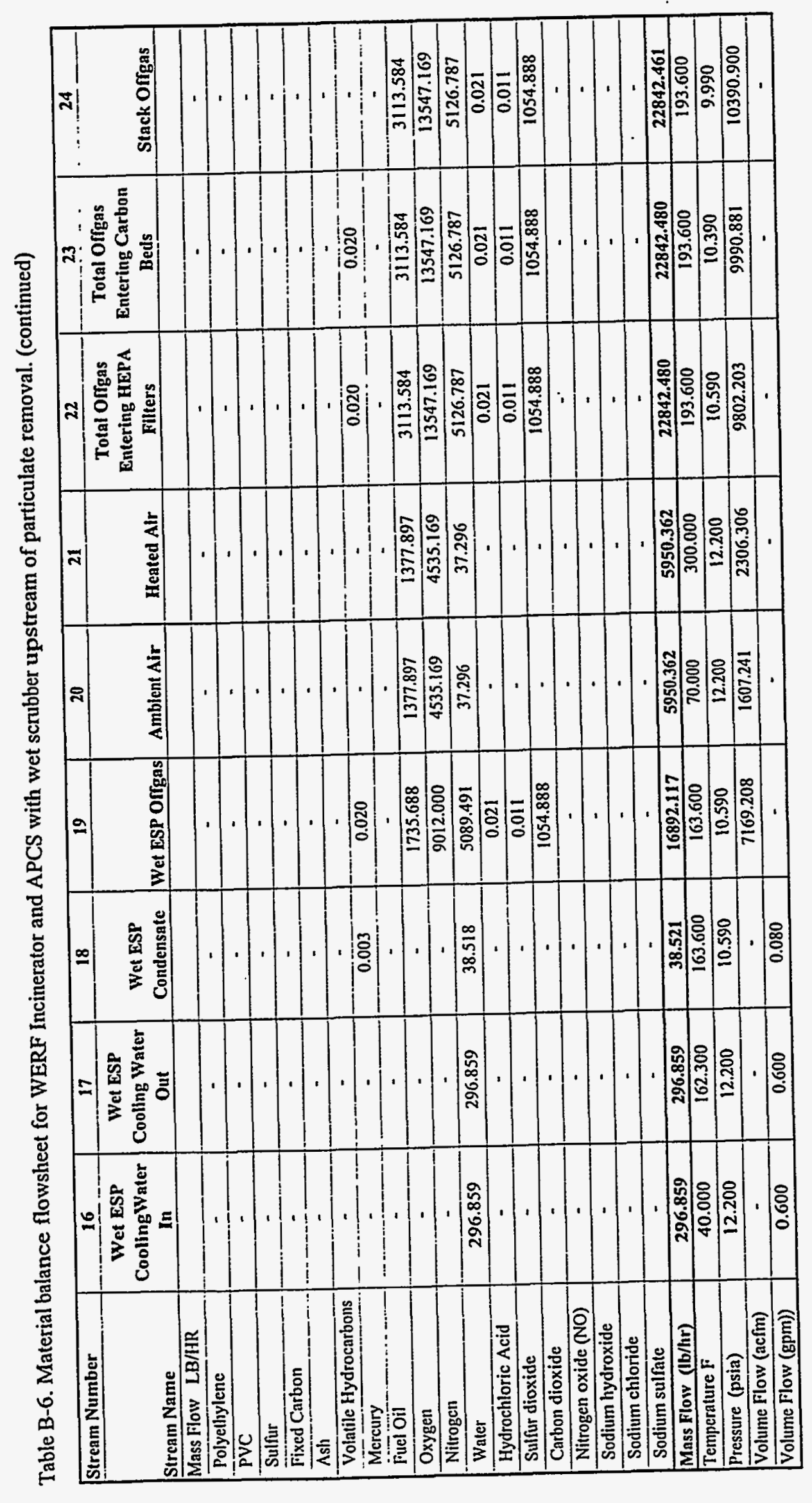

$\stackrel{\infty}{\infty}$ 


\section{B-6. REFERENCES:}

B-1 RCRA Part B Permit Application for the Idaho National Engineering Laboratory: Volume 9 - Waste Experimental Reduction Facility, Rev. 1, DOE/ID-10131, U.S. Department of Energy Idaho Field Office, Idaho Falls, ID, March 1994.

B-2 Idaho National Engineering and Environmental Laboratory Waste Experimental Reduction Facility (WERF) Incinerator Trial Burn: May and July 1997 - Volume III, INEEL/EXT-97-01180, LMITCO, Idaho Falls, ID, October 1997.

B-3 "Design \& Performance Characteristics of MERSORB ${ }^{\circledR}$ Mercury Adsorbents in Liquids and Gases", NUCON International, Inc., Columbus, $\mathrm{OH}$. 


\section{APPENDIX C DETAILED COST ESTIMATE}


Date: November 20, 1998

To: $\quad$ K. E. Kooda

MS 2414

6-8505

From: D. T. Peterson Bil

MS 3655

$6-2990$

Subject: WERF-MACT-FEASIBIITY STUDY - DTP-44-98

Cost Estimating has prepared the attached Planning/ROM Cost Estimate for the Waste Experimental Reduction Facility's (WERF) Maximum Achievable Control Technology (MACT) Feasibility Study. The Total Project Cost (TPC) for this project is $\$ 4,177,000$ which includes costs for construction, ED\&I, management costs, G\&A and PIF fees, procurement fees, Contingency, Management Reserve and other project cost. Please refer to the attached Cost Estimate Summary and Detail Sheets for cost breakdowns.

The Estimate is based on information provided in the Preliminary Design document, a tour of the proposed work area and assumptions as outlined on the attached Support Data Recapitulation Sheets. Any changes to the methodology used to prepare the estimate will have a significant effect on the cost estimate. The estimate is also based on preliminary information; any changes made during Conceptual Design should be reviewed by Cost Estimating.

If you have any questions or comments, please do not hesitate to contact me at 526-2990 or Office Vision-DD DTP.

DTP

Attachments

cc: Estimate File \#4157-Rev.1

D. T. Peterson Files

L. D. Torgerson

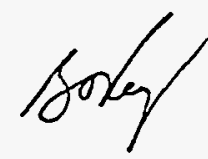


Lockheed Martin Idaho Technologies Co. PROAECT NAME: WERF MACT Feasibility Stuc'y

LOCATION 1: INEELWERF REQUESTOR:

K. Kooda
COST ESTIMATE SUMMARY

TYPE OF ESTMATE: Planning

PROJECT NO 4157

PREPAAED SY: D.T. Peterson

REPORT NAME: Cost Estimate Summary

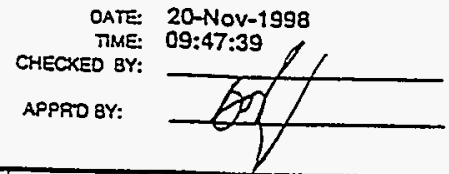

Total Incl Escalation

\begin{tabular}{|c|c|c|c|c|c|}
\hline $\begin{array}{l}\text { WBS } \\
\text { Element }\end{array}$ & Cost Estimate Element & $\begin{array}{c}\text { Total } \\
\text { Unescalated }\end{array}$ & Escalation & \multicolumn{2}{|c|}{$\begin{array}{c}\text { Total } \\
\text { Incl Escalation }\end{array}$} \\
\hline$\underline{1.1}$ & ENGINEERING, DESIGN AND INSPECTION & & & \multirow{3}{*}{\multicolumn{2}{|c|}{$\gg \quad \frac{\$ 389,461}{316,916}$}} \\
\hline 1.1 .1 & DESIGN ENGINEERING TITLE I \& II & 310,702 & 6,214 & & \\
\hline 1.1 .2 & QUALITY ASSURANCE & 69,755 & 2,790 & & \\
\hline 1.2 & MANAGEMENT COSTS & & & \multirow{3}{*}{\multicolumn{2}{|c|}{$\begin{array}{r}\$ 919,244 \\
855,824 \\
63,420\end{array}$}} \\
\hline 12.1 & PROUECT MANAGEMENT & 822,908 & 32,916 & & \\
\hline 1.2 .2 & CONSTRUCTION MANAGEMENT & 60,981 & 2,439 & & \\
\hline 1.3 & CONSTRUCTION & & & & $\$ 1,601,997$ \\
\hline 1.3 .1 & GENERAL CONDITIONS & 112,635 & 4,505 & & 117,140 \\
\hline 1.3 .2 & SITEWORK & 76,794 & 3,072 & \multicolumn{2}{|r|}{79,866} \\
\hline 1.3 .3 & CONCRETE & 30,975 & 1,237 & \multicolumn{2}{|r|}{32,152} \\
\hline 1.3 .5 & METALS & 82,512 & 3,300 & \multicolumn{2}{|r|}{85,812} \\
\hline 1.3 .9 & FINISHES & 13,532 & 541 & \multicolumn{2}{|r|}{14,073} \\
\hline 1.3 .15 & MECHANICAL & $1,159,870$ & 46,395 & \multicolumn{2}{|r|}{$1,206,265$} \\
\hline 1.3 .16 & ELECTRICAL & 45,394 & 1,816 & \multicolumn{2}{|r|}{47,210} \\
\hline 1.3 .13 & SPECIAL CONSTRUCTION & 18,730 & 749 & \multicolumn{2}{|r|}{19,479} \\
\hline 1.5 & G\&APIF & & & \multirow{3}{*}{\multicolumn{2}{|c|}{$\gg \quad \$ 48,059$}} \\
\hline 1.5 .1 & G\&AVPIF ADDER & 217,508 & 8,700 & & \\
\hline 1.5 .2 & PROCUREMENT FEES & 46,211 & 1,848 & & \\
\hline & $\begin{array}{l}\text { SUBTOTAL INCLUDING ESCALATION } \\
\text { PROJECT CONTINGENCY }\end{array}$ & $3,068,447$ & 116,522 & \multicolumn{2}{|c|}{$\gg \quad \$ 3,184,969$} \\
\hline & MANAGEMENT RESERVE & & & \multicolumn{2}{|r|}{$\$ 187,626$} \\
\hline & CONTINGENCY - & & & $\gg$ & $\$ 804,405$ \\
\hline & TOTAL ESTIMATED COST - & & & \multicolumn{2}{|c|}{$\gg \quad \$ 4.177 .000$} \\
\hline
\end{tabular}

PROJECT COST PARAMETERS

EDI AS A \% OF CONST. + GFE $=24.00 \%$ CONTINGENCY $=31.15 \%$ 


\section{COST ESTIMATE SUPPORT DATA RECAPITULATION}

Project Title: WERF MACT FEASIBILITY STUDY

Estimator: D. T. Peterson

Date: November 20, 1998

Estimate Type: Planning

File: 4157

Approved By:

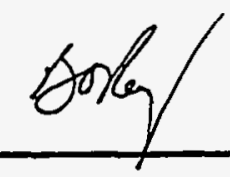

I. SCOPE OF WORK: Brief description of the proposed project.

This project will upgrade the existing Waste Experimental Reduction Facility's (WERF) off-gas system to meet anticipated Maximum Achievable Control Technology (MACT) requirements. The work scope will include the installation of a new evaporative off-gas cooler, dry sorbent injection system, fabric filter baghouse, carbon absorption system and two new off-gas exhaust fans.

II. BASIS OF THE ESTIMATE: Drawings, Design Report, Engineers Notes and/or other documentation upon which the estimate is originated.

The estimate is based on information provided by the requestor, flow diagrams of the proposed system upgrades and construction drawing of the existing system.

III. ASSUMPTIONS: Conditions statements accepted or supposed true without proof of demonstration. An assumption has a direct impact on total estimated cost.

- Contamination levels in the existing equipment and ducting that will be demolished will be low and would not have a significant impact on workers productivity.

- The equipment and materials that will be demolished will be removed in the largest pieces possible. Open ends of the demolished equipment and ducting will be covered and left at the WERF facility. Costs for size reduction, bagging, boxing, decontamination or transporting these materials has not been included in the estimate.

- It was assumed that the particulate loading in the ducts will be negligible and the ducts will be fabricated from 10 gauge welded carbon steel sheet metal conforming to SMACNA class one standards.

- Non-destructive testing (NDT) will be limited to liquid penetrant and visual inspection of welding. It was assumed that the subcontractor would be responsible for performing the NDT testing and the operating contractor would be responsible for the acceptance of the tests. It was also assumed that the ducting would be leak tested using the pressure decay method.

- Modification to the existing stack or stack sampling systems will not be required.

- Structural modifications to the existing floors will not be required to support the new equipment. 


\section{COST ESTIMATE SUPPORT DATA RECAPITULATION}

- Continued -

Project Title: WERF MACT FEASIBIITY STUDY

File: 4157

Page 2 of 3

- It was assumed that only new breakers would need to be installed in the existing MCC to support the new equipment.

- The existing conduit and wire circuits that are currently supporting the existing fans would be too small for the new fans. Costs have been included to route new conduit and wire runs to support $130 \mathrm{AMP}$.

- A new flow control system will be installed to maintain required air flows.

- Isolation damper will be installed on either side of the new fans.

- Flexible duct connections will be installed on fan inlet and outlets.

- An independent design review will be required on this project.

IV. CONTINGENCY GUIDELINE IMPLEMENTATION: The percentage used for contingency as determined by the contingency allowance guidelines can be altered to reflect the type of construction and conditions that may impact the total estimated cost.

Thirty- percent contingency has been included in the estimate. The estimate is based on very preliminary information. Facility or equipment requirements could change during design, differing conditions may be encountered during design, or construction and regulatory changes could increase or change-all of which could have a significant impact on the project cost. The level of contingency is within the accepted range for an estimate at this stage of development. Please refer to the attached contingency analysis sheet for details.

\section{OTHER COMMENTS/CONCERNS SPECIFIC TO THE ESTIMATE}

- Costs reflected on the detail sheets are direct costs for materials and labor. Costs for corresponding divisions shown on the Cost Estimate Summary sheet include all applicable indirect costs (overhead, profit, bonds and sales tax on materials).

- Costs for the current General and Administrative allowance (G\&A), procurement, and Performance Incentive Fee (PIF) have been included in the estimate. Please refer to the attached work sheet for details.

- The estimate is based on present day costs that have been escalated to the anticipated mid-point of each activity. It was assumed Conceptual Design to begin in March of CY 1999.

- Any moisture-laden air leaving the evaporative cooler could have a seriously corrosive impact on the carbon steel ducting and equipment. If it is determined that these components should be stainless steel or other corrosive resistant alloys, costs will increase dramatically from what has been included in the estimate. 


\begin{tabular}{|lc|}
\hline \multicolumn{2}{|c|}{ COST ESTIMATE SUPPORT DATA RECAPITULATION } \\
Project Title: WERF MACT FEASIBIITY STUDY \\
File: 4157 & Page 3 of 3 \\
\hline
\end{tabular}

- Total project duration is anticipated to be twenty-one months, and is broken down as follows:

$\begin{array}{ll}\text { Pre-Conceptual Design } & 2 \text { months } \\ \text { Conceptual Design } & 4 \text { months } \\ \text { Title Design } & 6 \text { months } \\ \text { Procurement } & 1 \text { month } \\ \text { Construction } & 6 \text { months } \\ \text { SO Testing \& Start-Up } & 2 \text { months }\end{array}$

- Project Management and Other Project costs were provided by Mr. K. E. Kooda and L. D. Torgerson. 


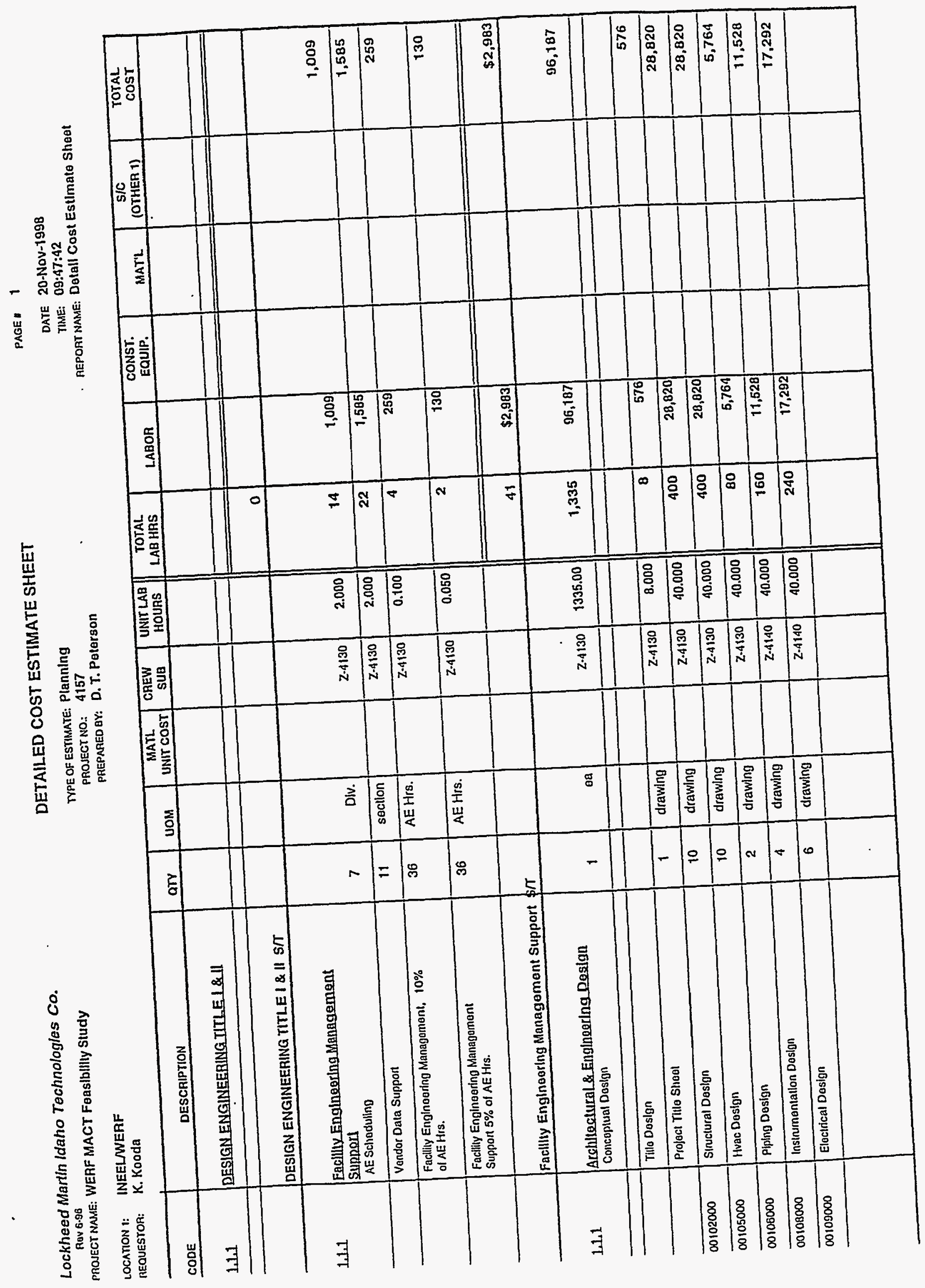




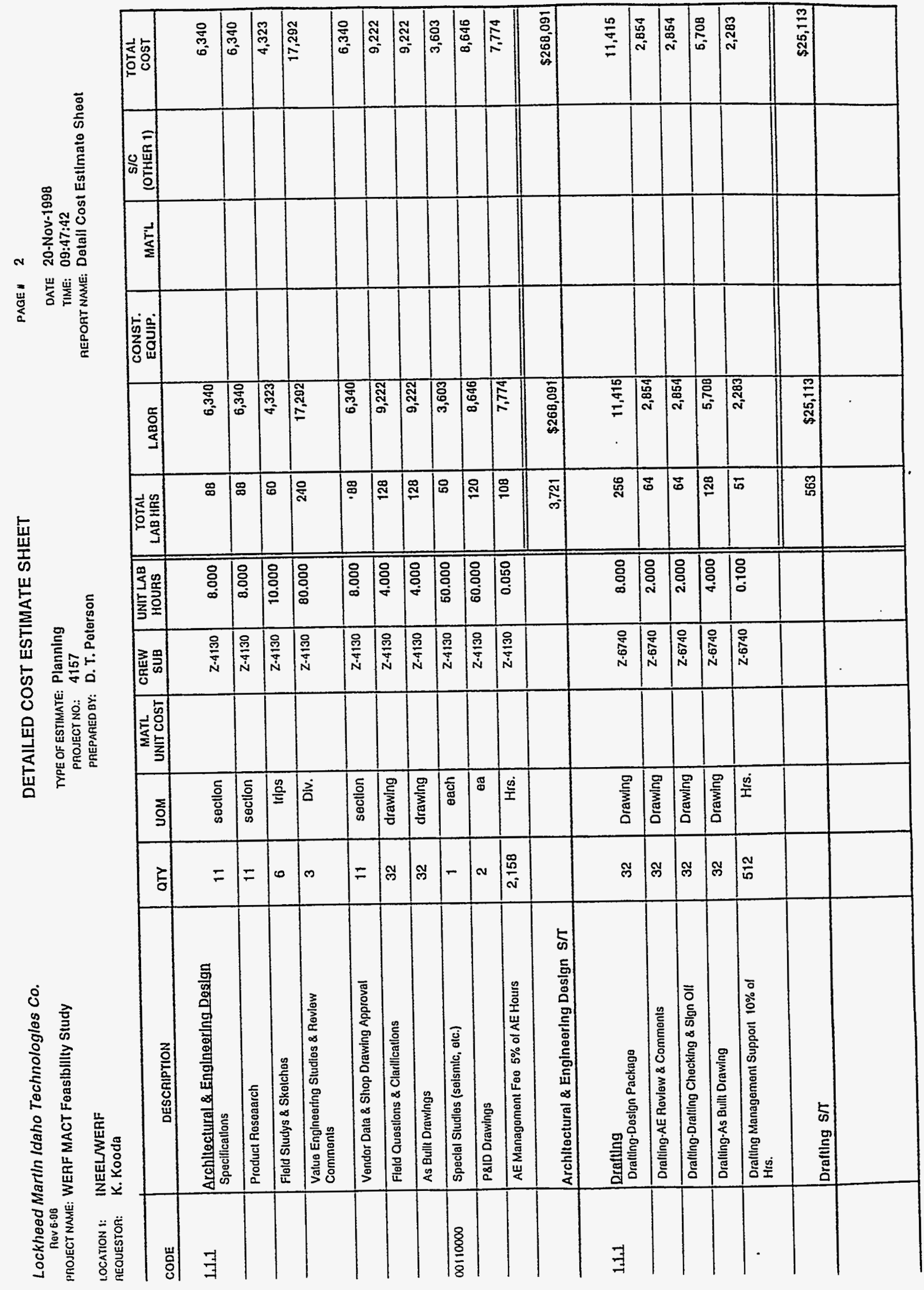




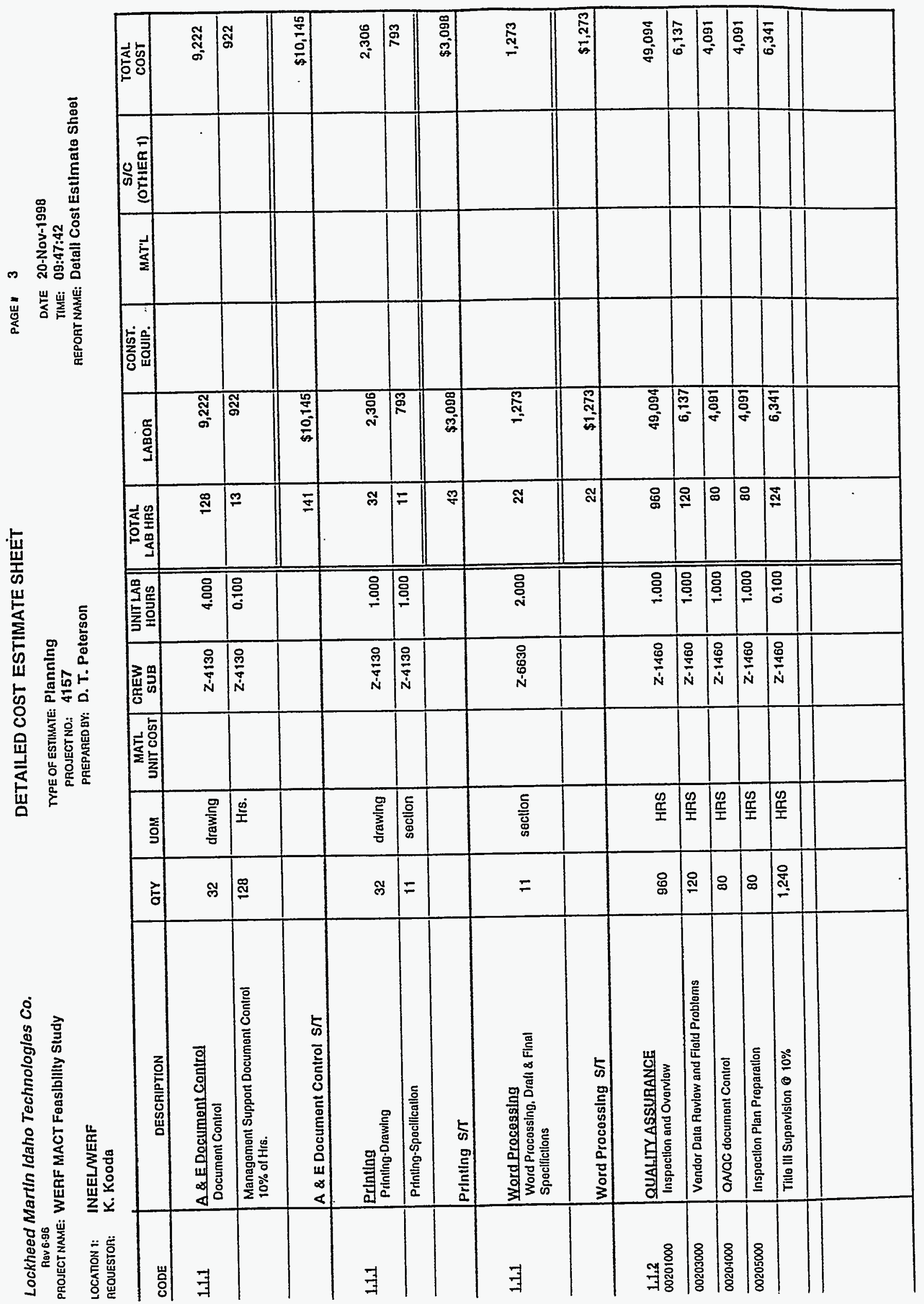




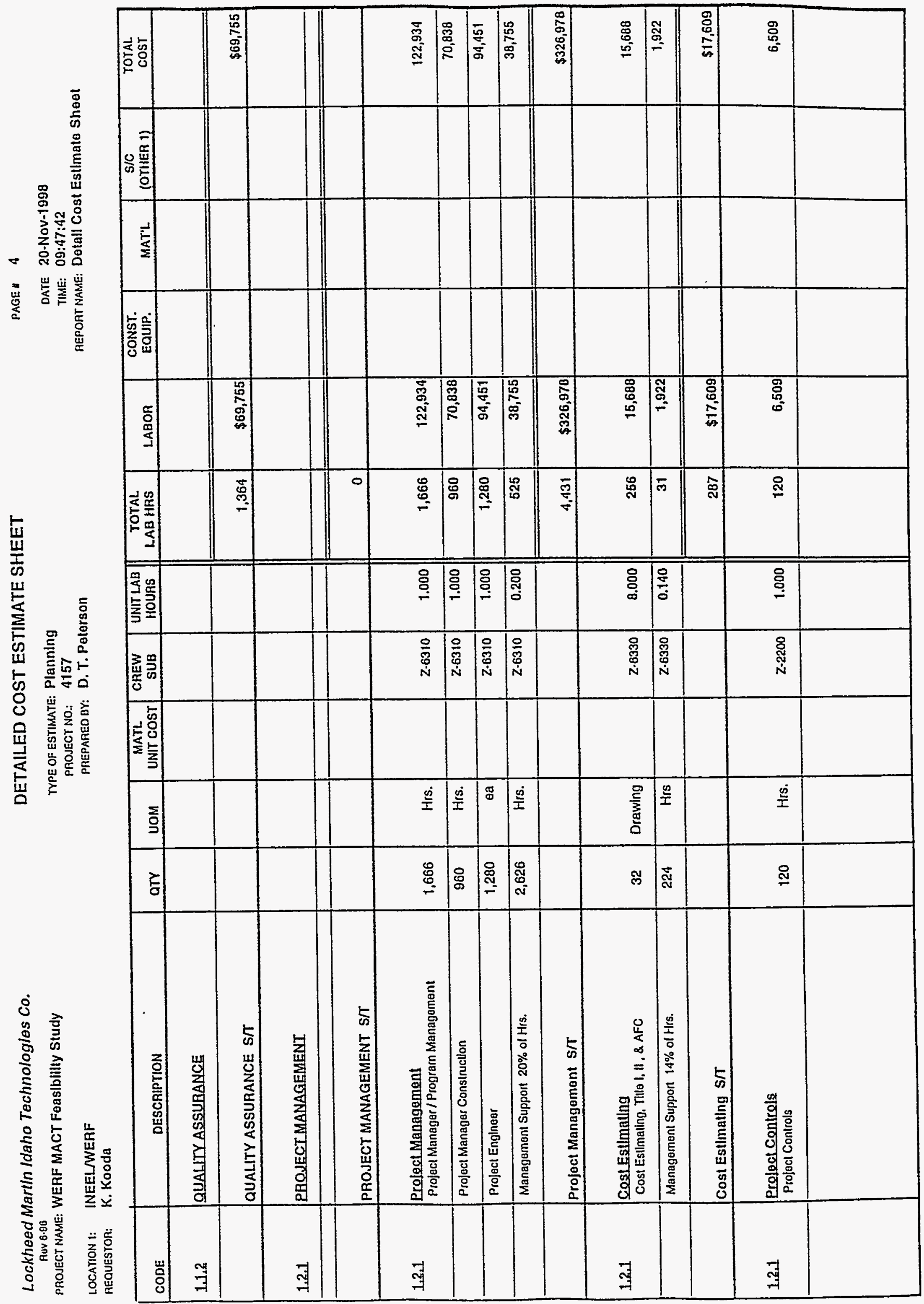




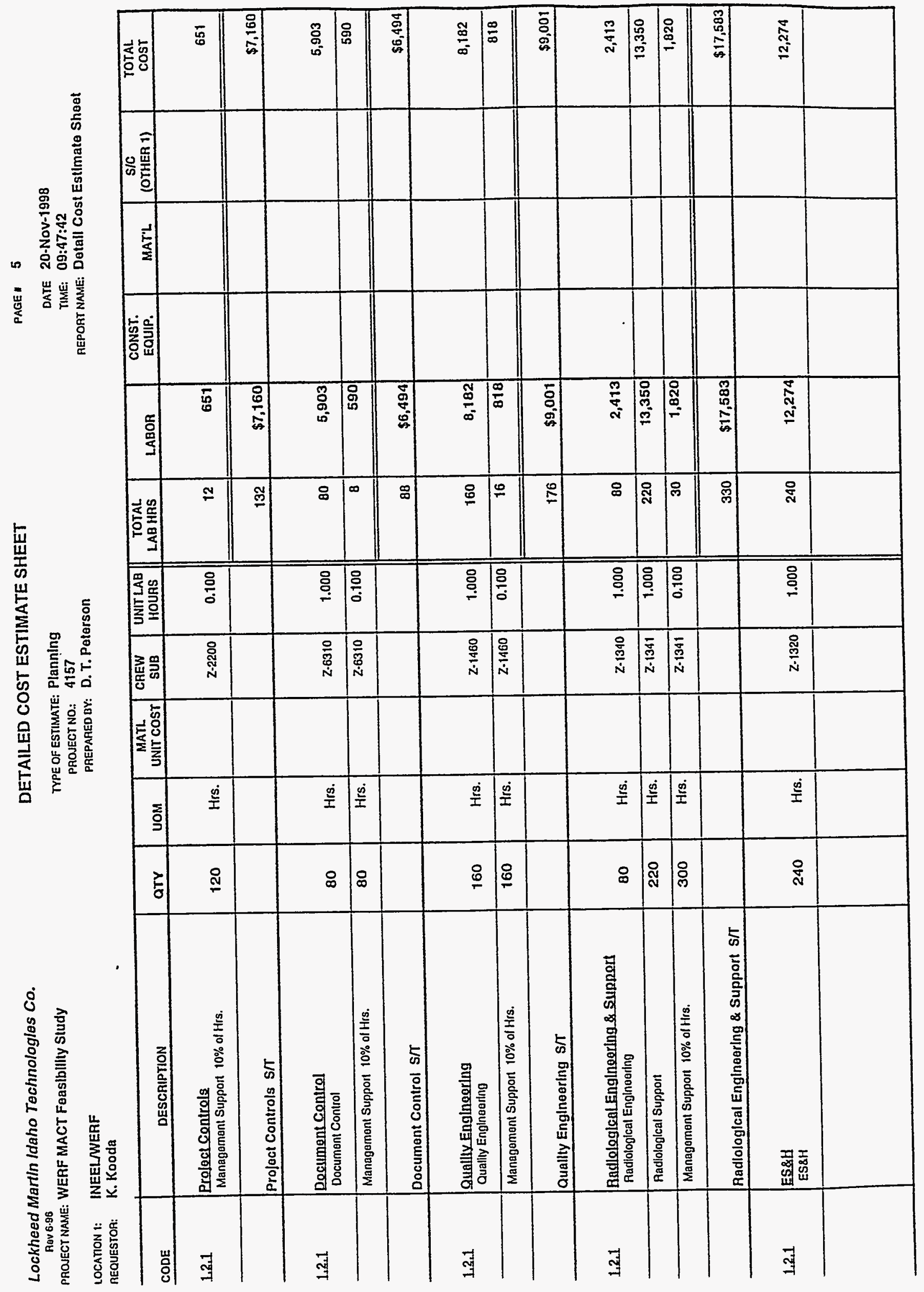




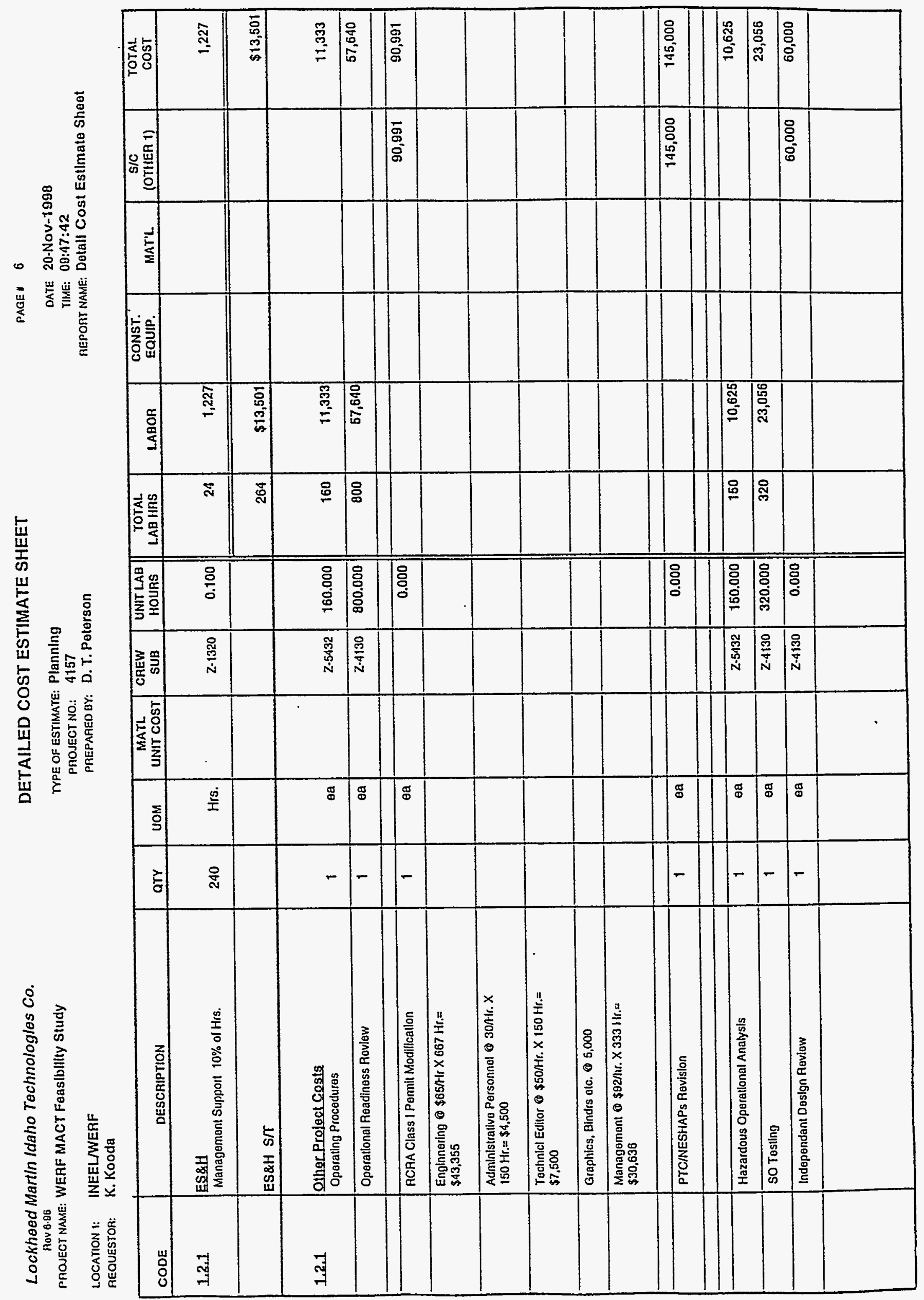




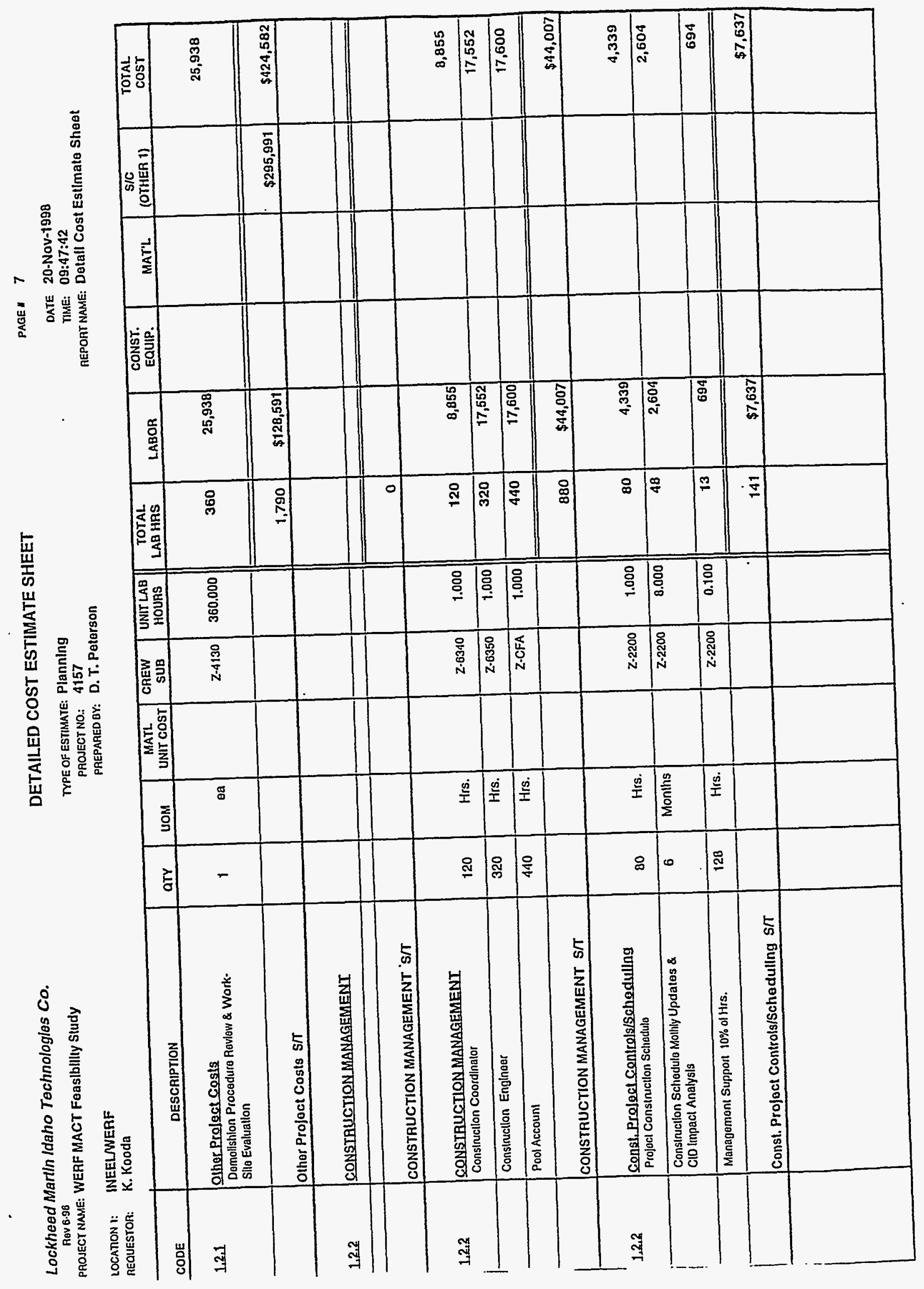




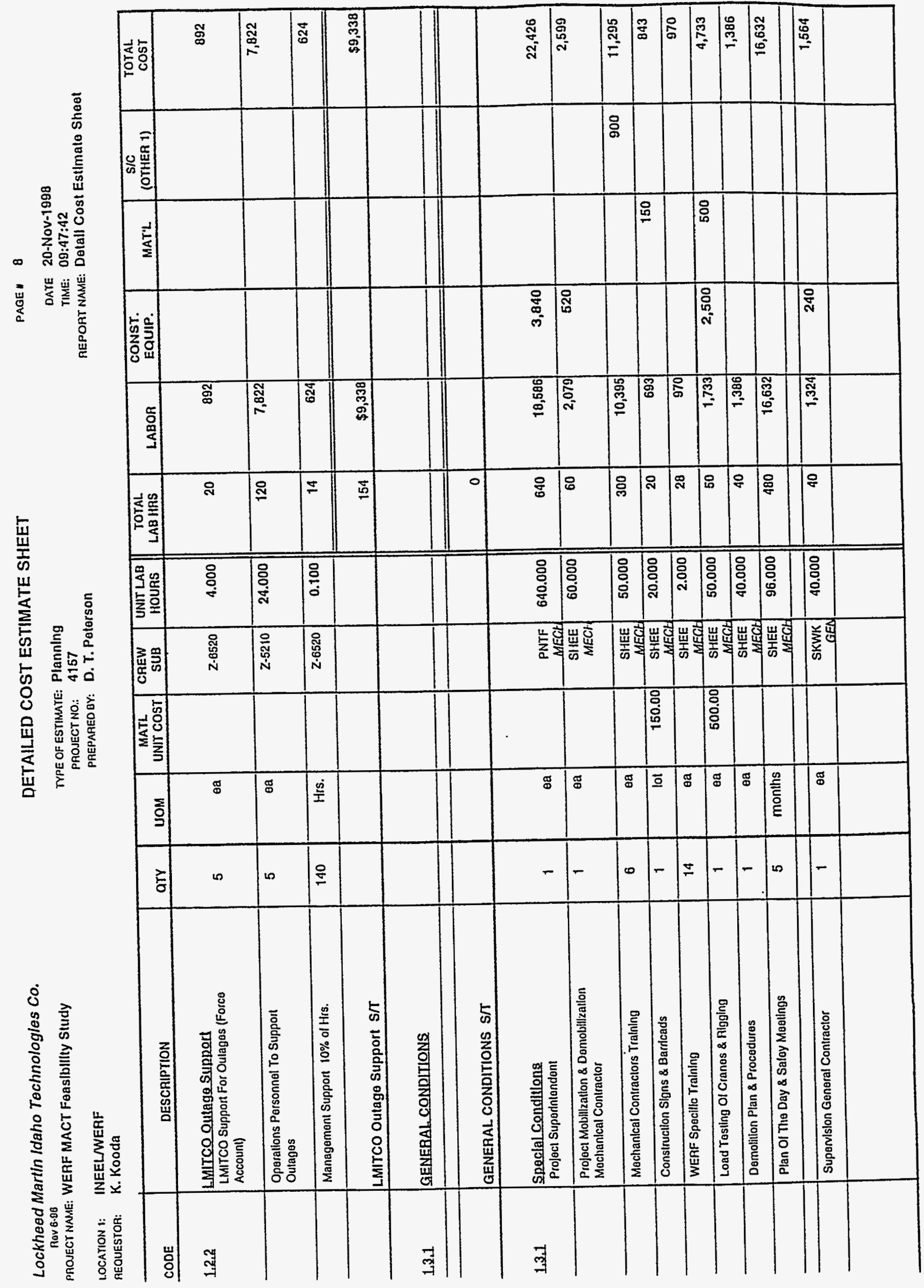




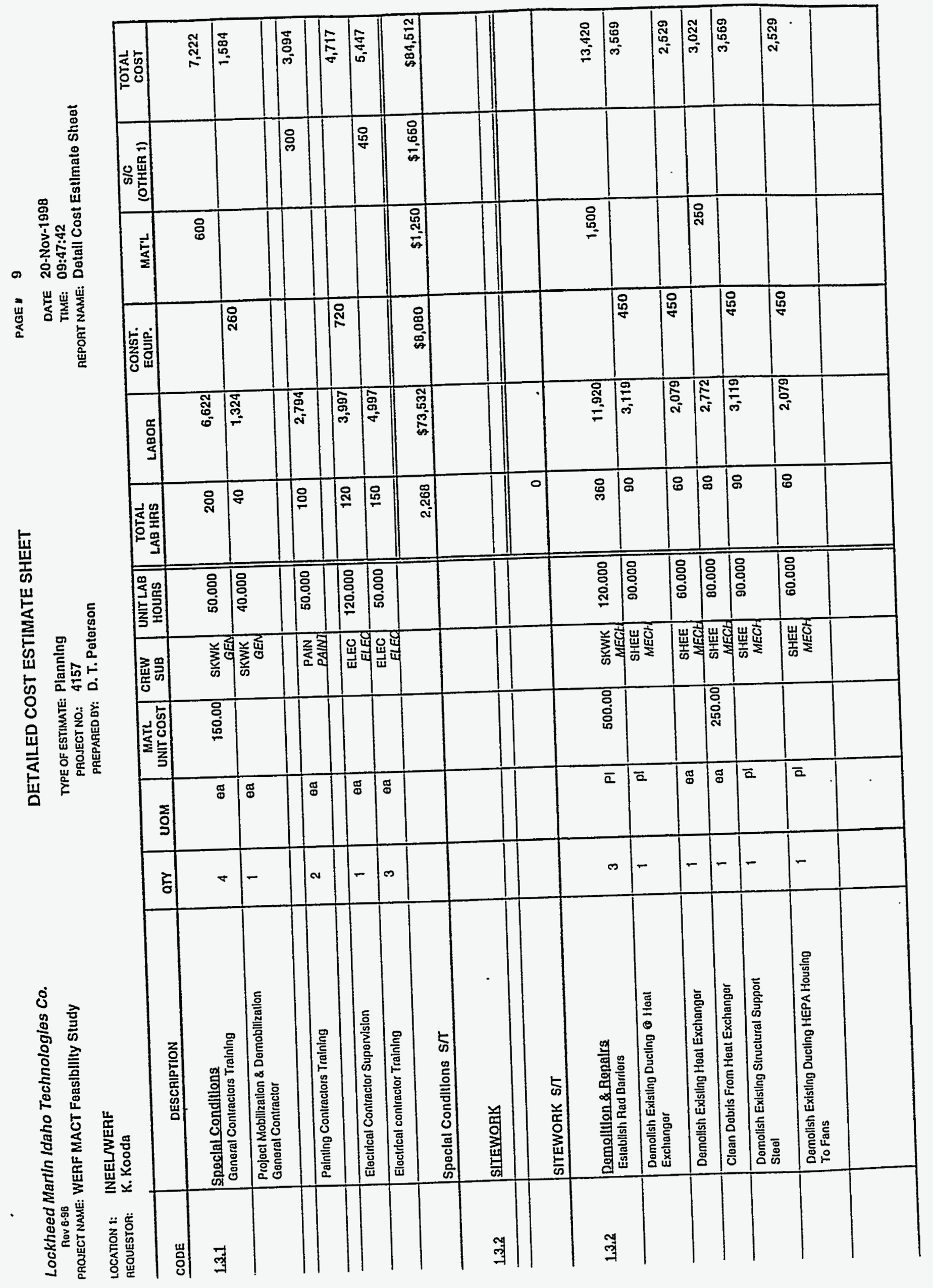




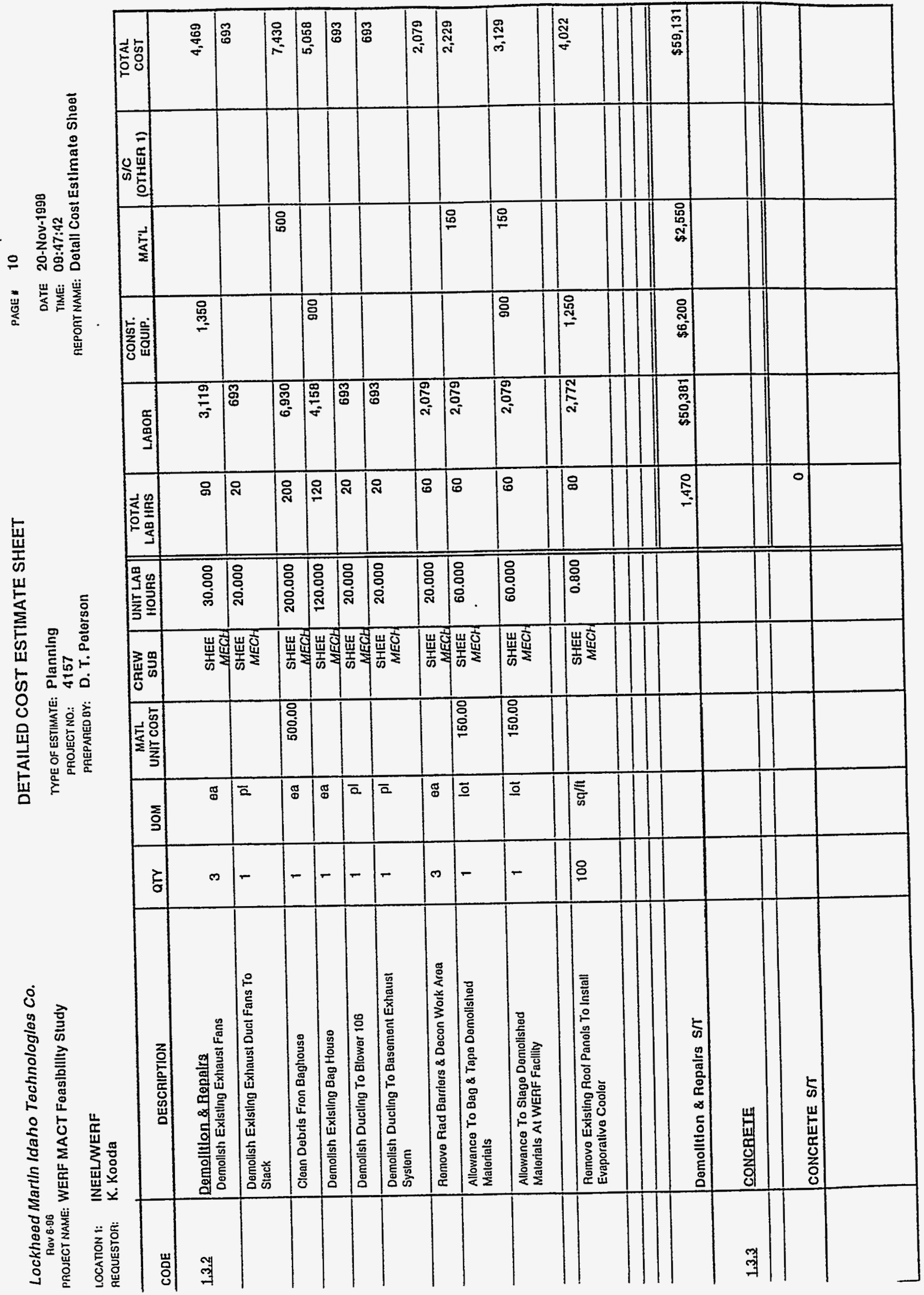




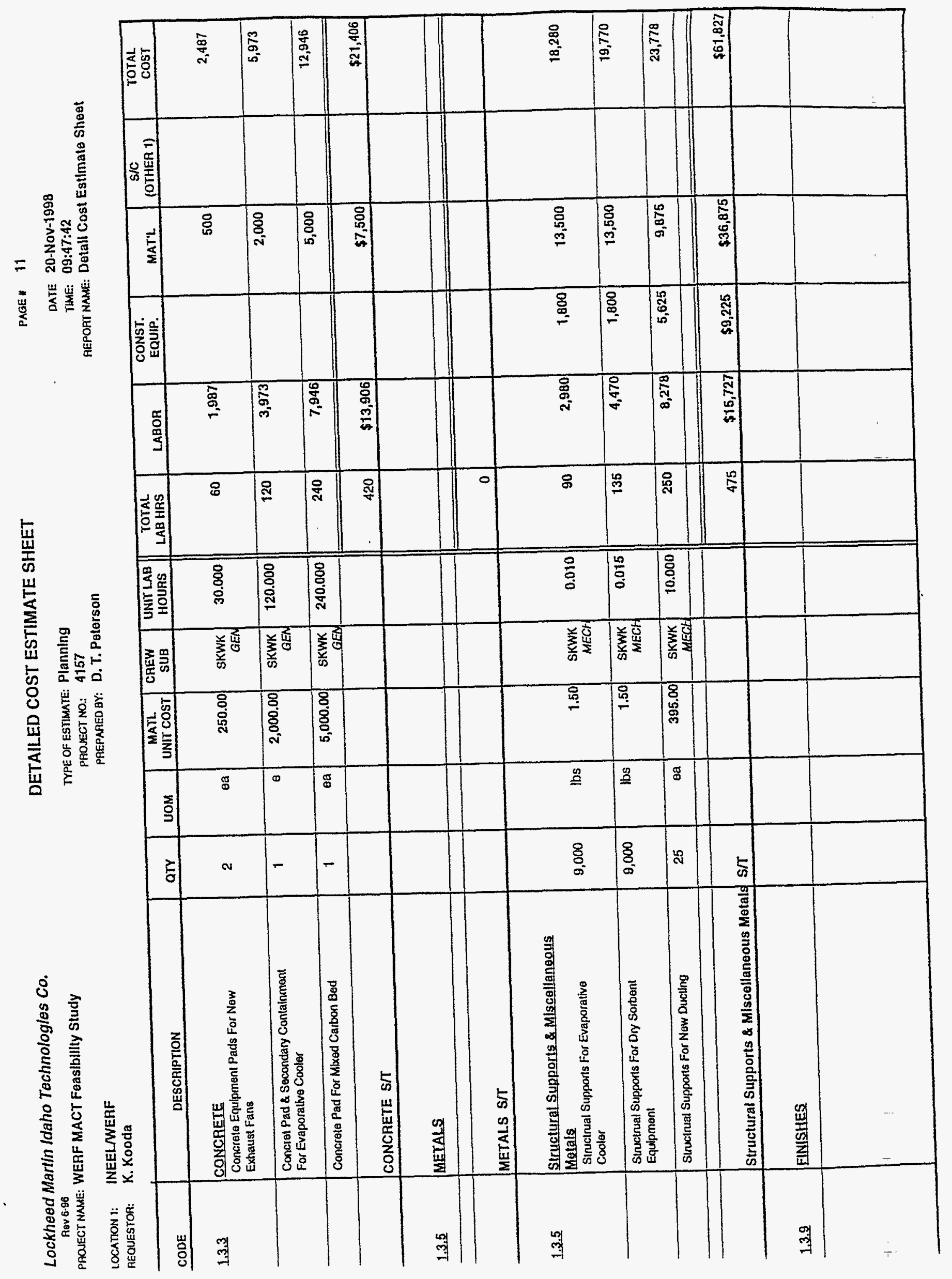




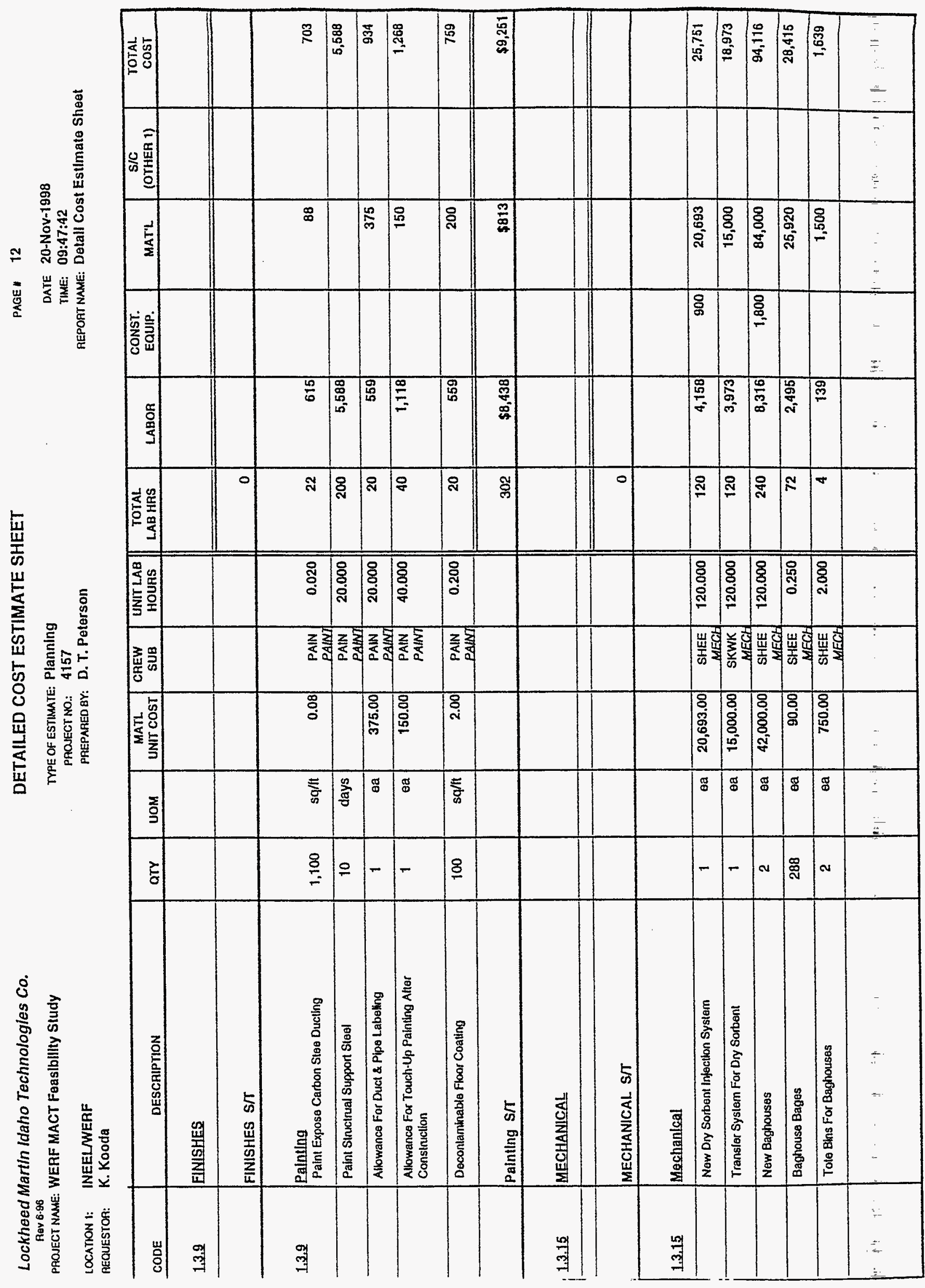




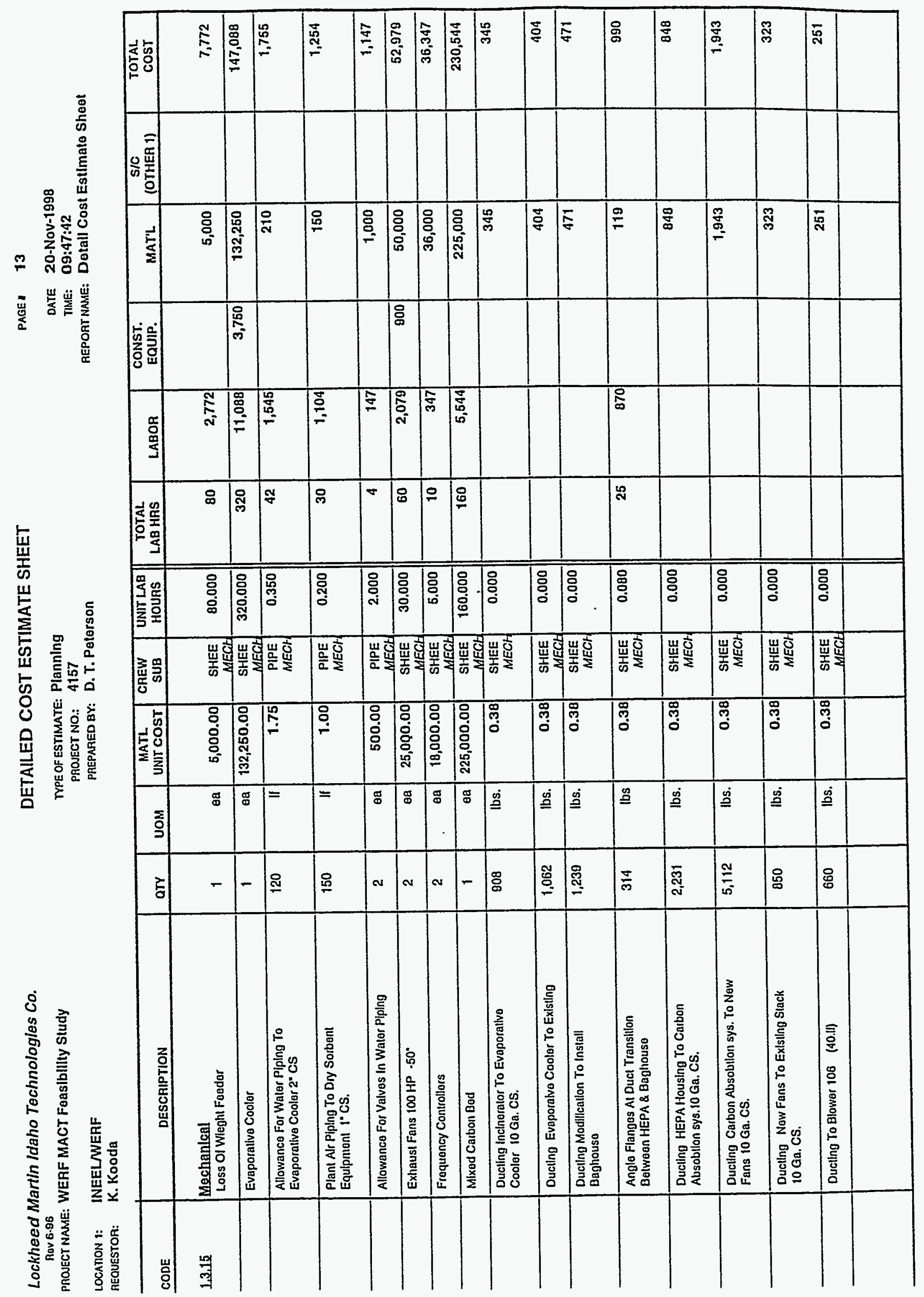




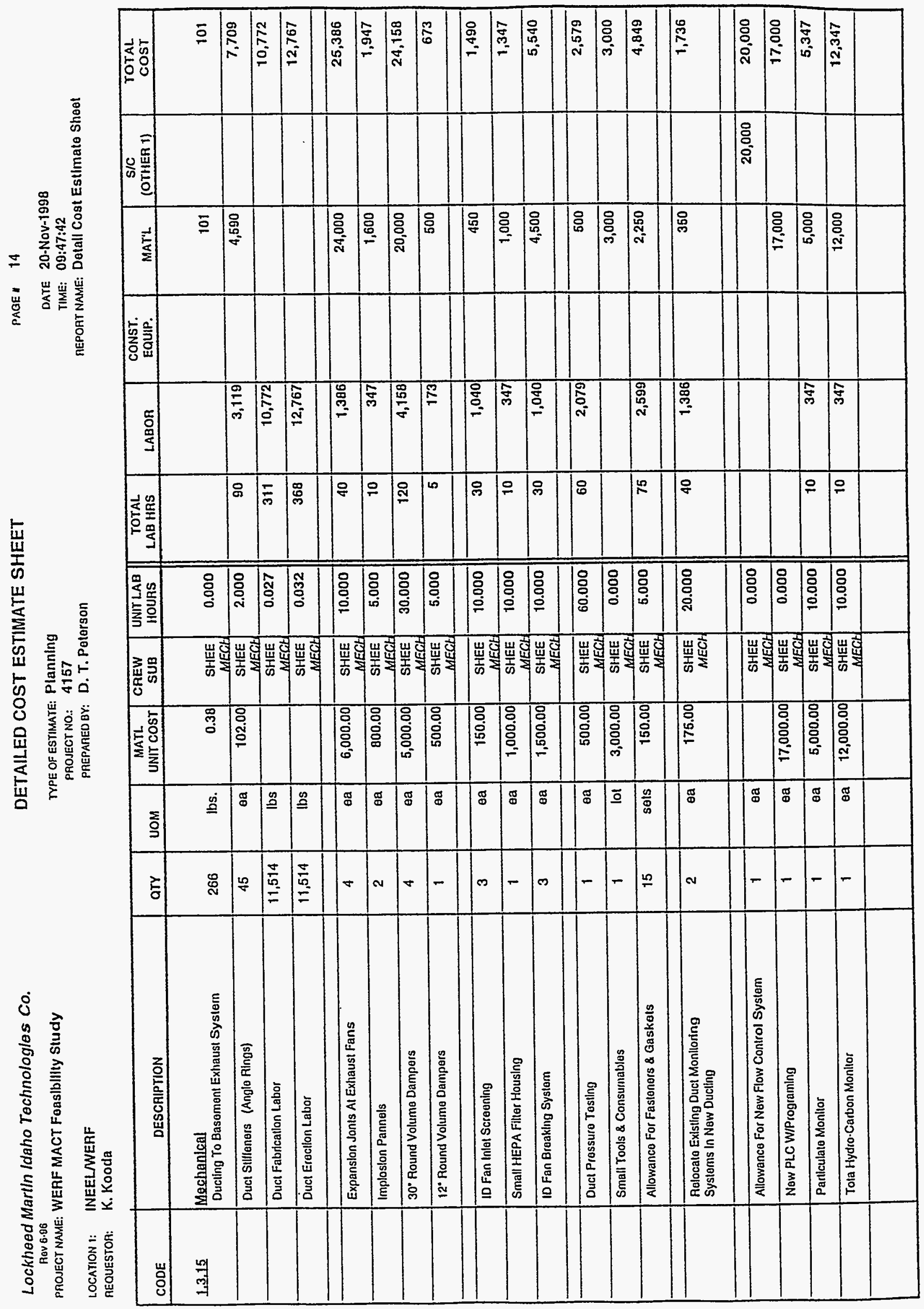




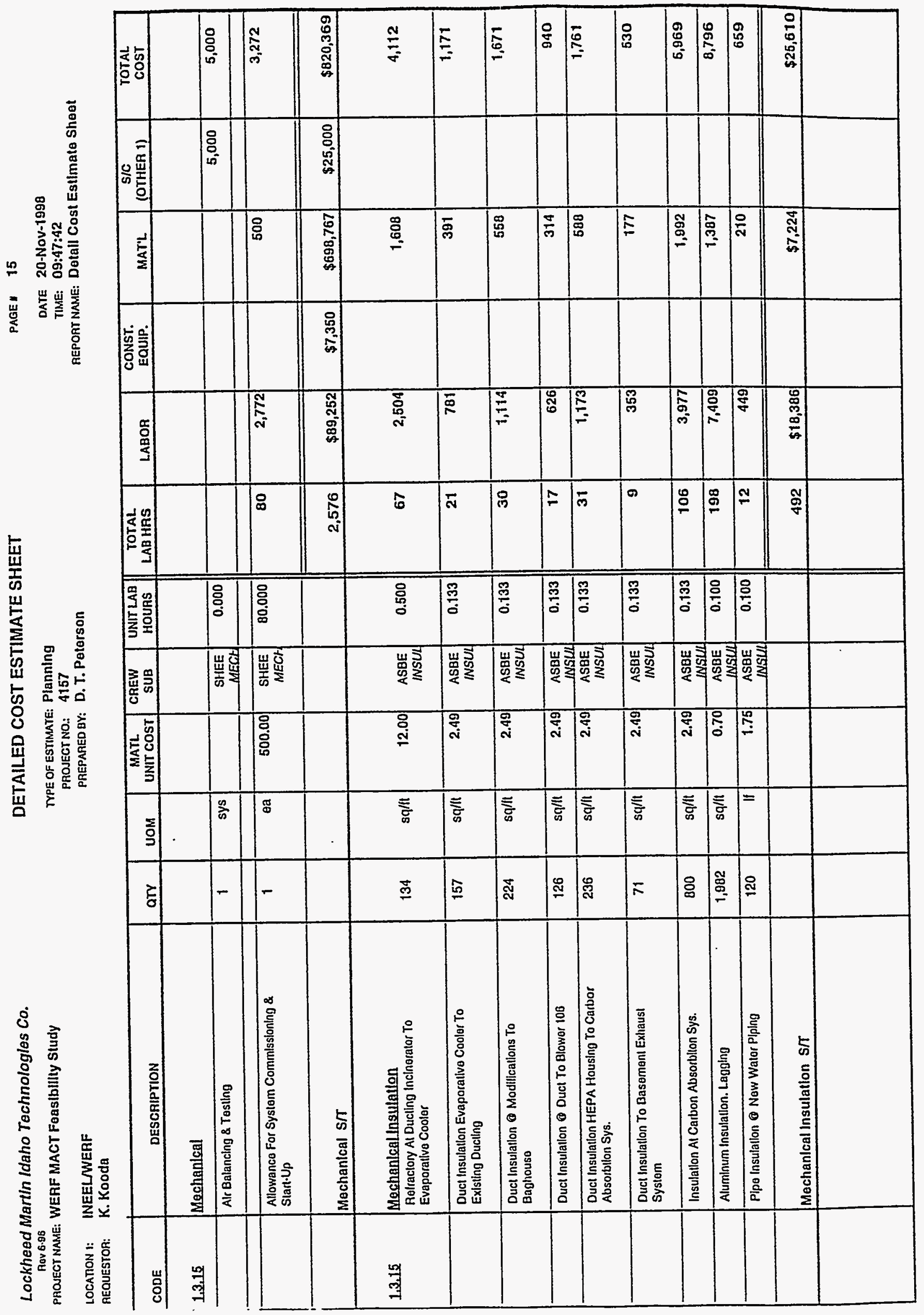




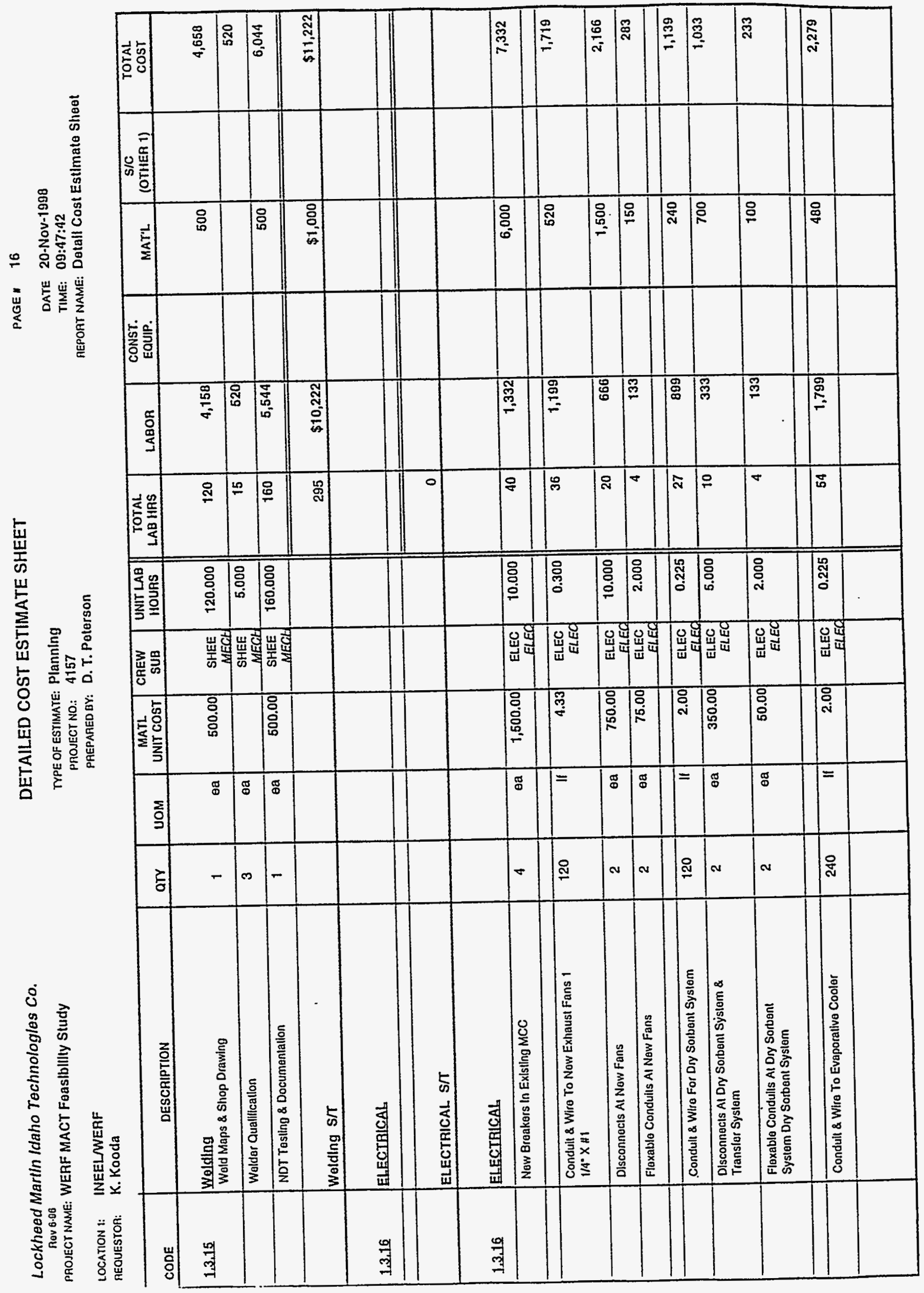




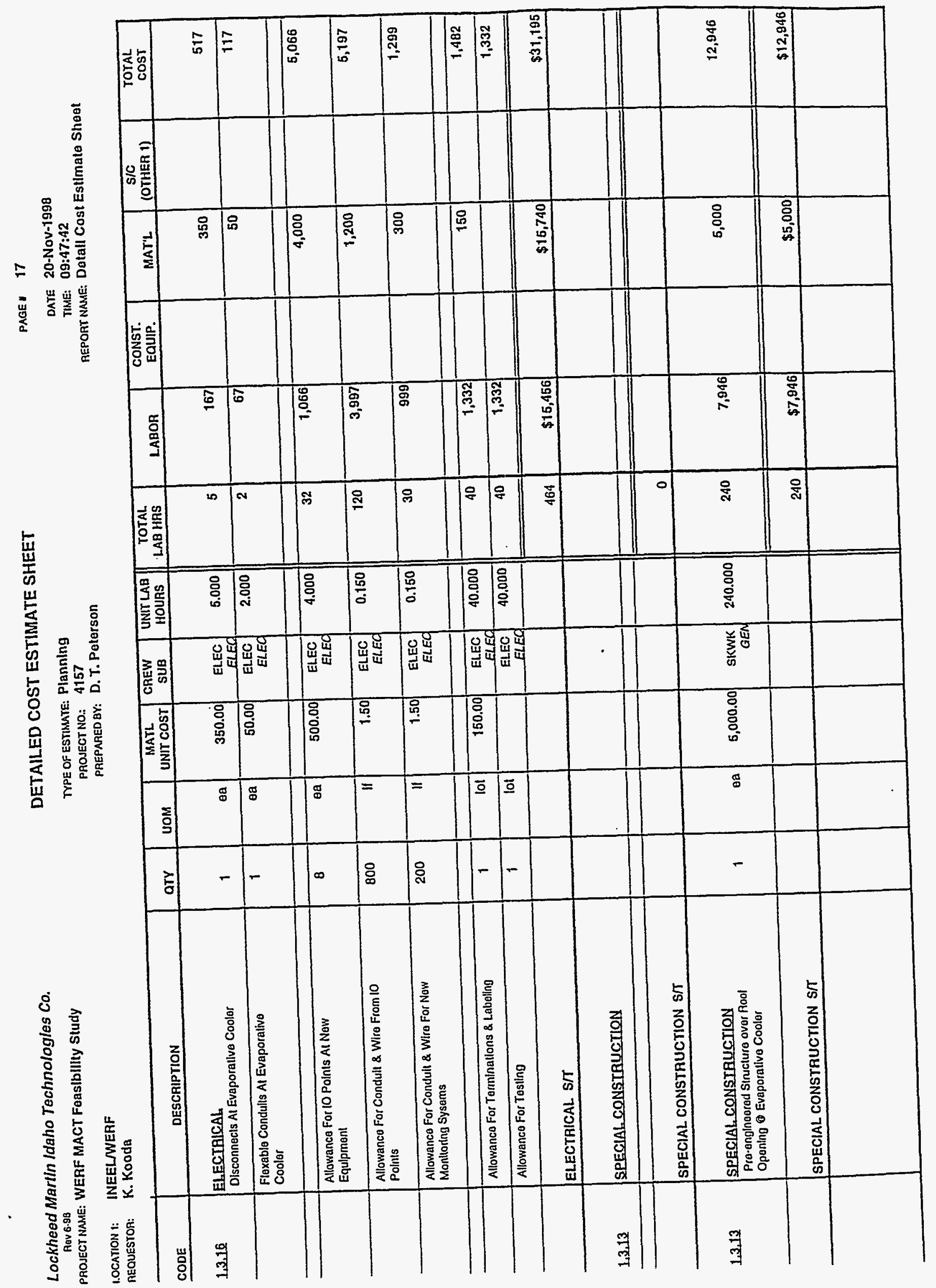




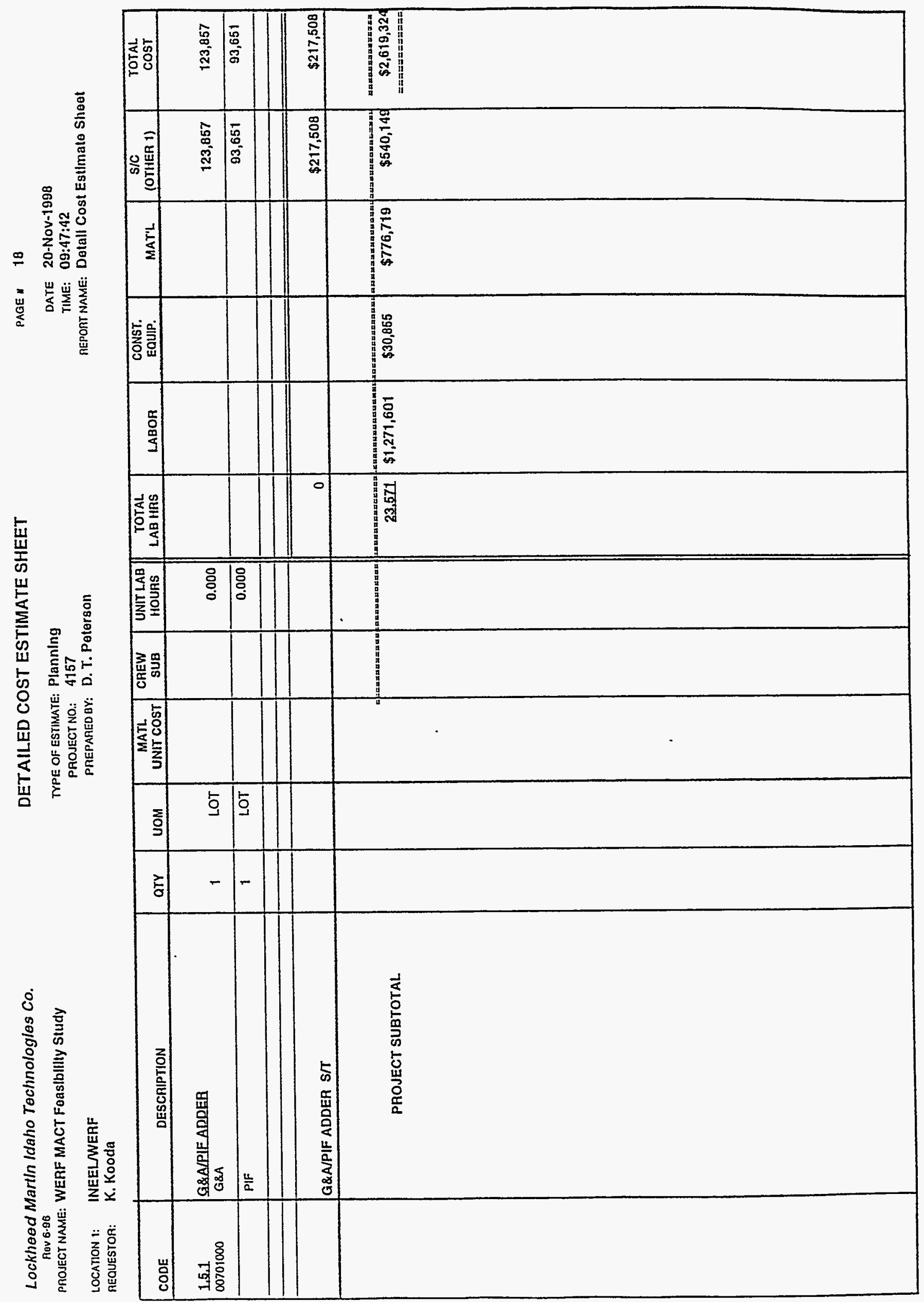


Lockheed Martin Idaho Technologies Co. PROSECT NAME: WERF MACT Feasibility Study

LOCATION 1: INEELNWERF REOUESTOR:
K. Kooda
CONTINGENCY ANALYSIS

TYPE OF ESTMATE: Planning

FROECT NO: 4157

D. T. Peterson
OATE: 20-NOV-1998

TIME: 09:47:34

REPORT NAuE: Contingency Analysis

\begin{tabular}{|c|c|c|c|c|c|c|c|c|c|c|c|}
\hline & PROB & BLE \% VARLAT & ON & & & & & & $\begin{array}{l}\text { PRO } \\
\text { CONT }\end{array}$ & $\begin{array}{l}T \\
\text { ENCY }\end{array}$ & SUMMARY \\
\hline $\begin{array}{l}\text { WBS } \\
\text { Element }\end{array}$ & Cost Estimate Element & Total Cost w/o & $\begin{array}{c}\text { \% Total } \\
\text { Cost }\end{array}$ & & $\begin{array}{l}\% \text { Var. } \\
\text { m Est }\end{array}$ & WL $\%$ & Prob. & Comtingency & $\%$ & Cost & Total Cost \\
\hline & & Contingency & & - & + & - & + & & & & by gement \\
\hline 1.1 .1 & DESIGN ENGINEERING TILE $1 \& \|$ & 310,702 & 9.76 & 10 & 35 & 0.98 & 3.41 & $2.975 \%$ & $9.60 \%$ & 95,214 & 405,916 \\
\hline 1.1 .2 & QUALTYY ASSURANCE & 69.755 & 219 & 10 & 30 & 0.22 & 0.66 & $0.569 \%$ & $1.84 \%$ & 18,222 & $87,9 \pi$ \\
\hline 121 & PROUECT MANAGEMENT & 822,308 & 25.34 & 10 & 40 & 258 & 10.33 & $9.043 \%$ & $29.17 \%$ & 289,386 & $1,112,294$ \\
\hline 1.22 & CONSTRUCTION MANAGEMENT & 60,981 & 1.91 & 10 & 30 & 0.19 & 0.57 & $0.498 \%$ & $1.57 \%$ & 15,930 & 76,911 \\
\hline 1.3 .1 & GENERAL CONDITIONS & 112,635 & 3.54 & 10 & 40 & 0.35 & 1.41 & $1238 \%$ & $3.99 \%$ & 39,510 & 152.245 \\
\hline 1.3 .2 & SITENORK & 76,794 & 241 & 5 & 50 & 0.12 & 1.21 & $1.073 \%$ & $3.46 \%$ & 34,336 & 111,130 \\
\hline 1.3 .3 & CONCAETE & 30,915 & 0.97 & 10 & 35 & 0.10 & 0.34 & $0.296 \%$ & $0.95 \%$ & 9,474 & 40.389 \\
\hline 1.3 .5 & METALS & 82,512 & 2.59 & 10 & 35 & 0.26 & 0.97 & $0.790 \%$ & $255 \%$ & 25,296 & 107,798 \\
\hline 1.3 .9 & FINISHES & 13,332 & 0.42 & 5 & 40 & 0.02 & 0.17 & $0.151 \%$ & $0.49 \%$ & 4,827 & 18,359 \\
\hline 1.3 .15 & MECHANICAL & $1,159,370$ & 36.42 & 10 & 40 & 3.54 & 14.57 & $12746 \%$ & $47.12 \%$ & 407,883 & $1,557,753$ \\
\hline $1,3.16$ & ELECTRICAL & 45,394 & 1.43 & 5 & 35 & 0.07 & 0.50 & $0.442 \%$ & $1.43 \%$ & 14,139 & 59,533 \\
\hline 1.3 .13 & SPECIAL CONSTRUCTION & 18,730 & 0.59 & 0 & 0 & 0.00 & 0.00 & $0.000 \%$ & $0.00 \%$ & 0 & 18,730 \\
\hline 1.5 .1 & G\&AVIF ADDEA & 217,508 & 6.33 & 5 & 10 & 0.34 & 0.63 & $0.580 \%$ & $1.87 \%$ & 18,575 & 236.084 \\
\hline 1.52 & PROCUREMENT FEES & 46,211 & 1.45 & 5 & 10 & 0.07 & 0.15 & $0.123 \%$ & $0.40 \%$ & 3,947 & 50,158 \\
\hline & ESCALATION & 115,522 & 3.66 & 10 & 20 & 0.37 & 0.73 & $0.522 \%$ & $207 \%$ & 75.201 & 137.723 \\
\hline & SUBTOTAL & $3,184,969$ & 100.00 & & & & & $31.147 \%$ & & $\cdot$ & \\
\hline & CALCULATED CONTINGENCY & 992,019 & & & & & & & & & \\
\hline & RESULTANT TEC & $4,176,988$ & & & & & & & & & \\
\hline & ROUNDED TEC & $4,177,000$ & & & & & & & & & \\
\hline & PROJECT CONTINGENCY & 992,031 & & & & & & $31.15 \%$ & & & \\
\hline & MANAGEMENT RESEAVE & 187,626 & & & & & & & & & \\
\hline & CONTINGENCY & 804.405 & & & & & & & & & \\
\hline & TOTALESTIMATED COST & $4,177,000$ & & & & & & & & 992,031 & $4,177,000$ \\
\hline
\end{tabular}

CONFIDENCE LEVEL AND ASSUMED RISKS:

The Lockheed Idaho Technologies Co. Cost Estimate Contingency Analysis Model is based on the applied contingency and the assumptions upon which the estimate was predicated. The model is applied with a suggested risk level of $18 \%$ and a level of confidence of $90 \%$ the estimate will fall within the bid range. The Contingency Analysis is based on a weighted average to provide a

$90 \%$ probability of underun and a $10 \%$ probabitity of overun.
CONTINGENCY ANALYSIS GUIDE BY TYPE OF ESTIMATE

Guidelines established by DOEIFM 50 , Cost Estimating Guide, Vol. $\delta$,

Cost Guide, and as presented in the INEL Cost Estimating Guide. PLANNING $20 \%-30 \%$ Experimental/Special Conditions............. Up to 50\% Conceptual Experimental/Special Conditions............. Up to $40 \%$ TITLE! TITL IIVAFC
$10 \%-20 \%$

$5 \%-15 \%$ Market Conditions 


\section{G\&A/PIF ADDER CALCULATION SHEET}

PROCUREMENT FEE: CONSTRUCTION $=$
GFE $=$

FEE @ $2.5 \%=$
$\$ 1,540,381$

Subtotal $\$ \frac{\$ 1,540,381}{}$

G\&A @ 23\% (with a ceiling of $\$ 500,000$ imposed per year CONSTRUCTION \$ OR CEILING •\# OF YEARS YEARS OF CONST. = $1 \$ 500,000$

GFE = $\$ 1,540,381 \cdot 0.025=$

PROCUREMENT FEE =

Subtotal $\$ 38,510$ $\$ 538,510$

FEE @ $23 \%=$ $\$ 538,510 * 0.23=$ $\$ 123,857$

PIF @ 5.5\%

\begin{tabular}{lr} 
CONSTRUCTION $=$ & $\begin{array}{r}\$ 1,540,381 \\
\text { GFE }=\end{array}$ \\
PROCUREMENT FEE $=$ & $\$ 0$ \\
G\&A $=\quad \begin{array}{r}\$ 38,510 \\
\end{array} \quad$ Subtotal & $\$ 123,857$ \\
\hline
\end{tabular}

FEE @ $5.5 \%=$ 


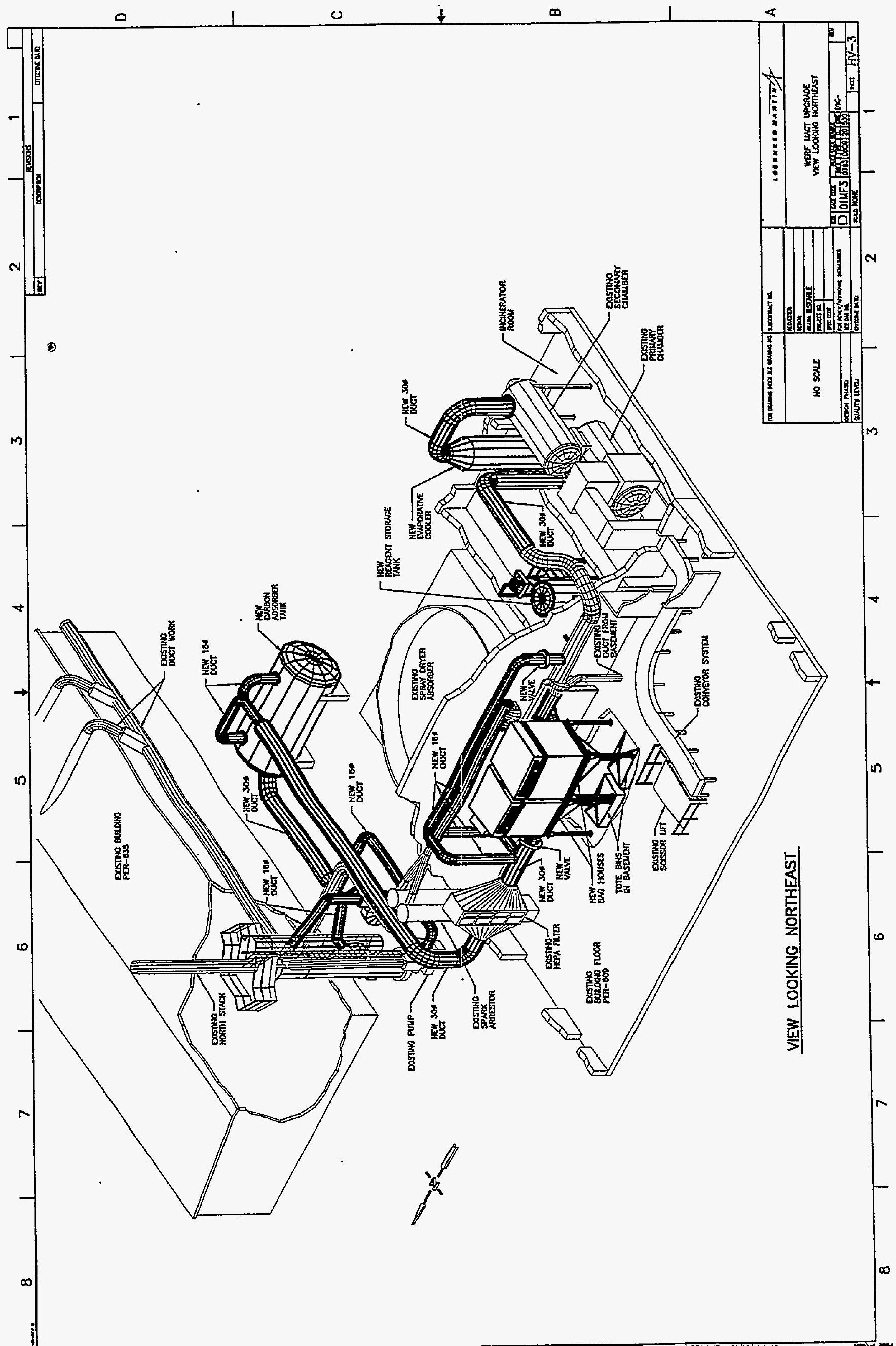




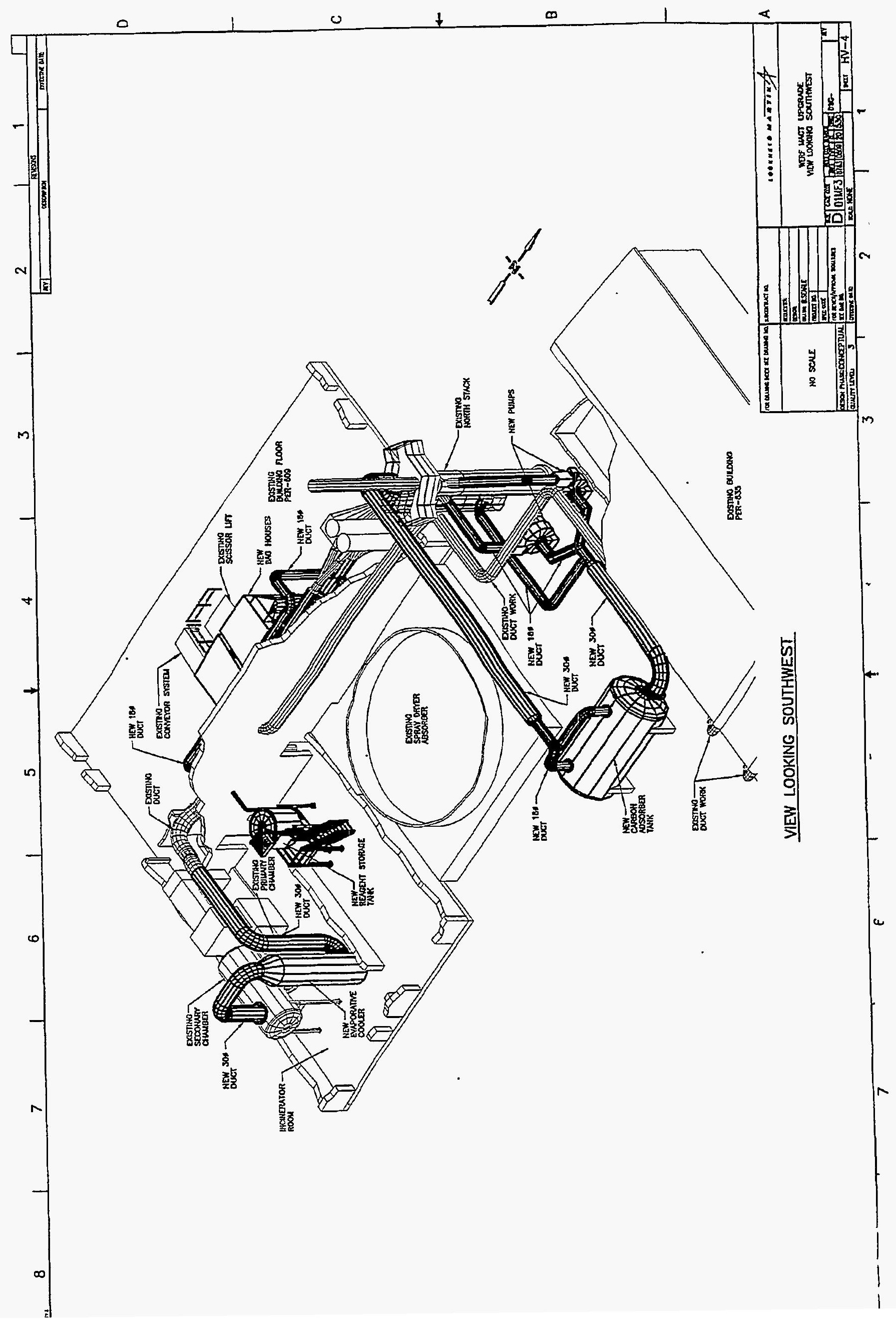




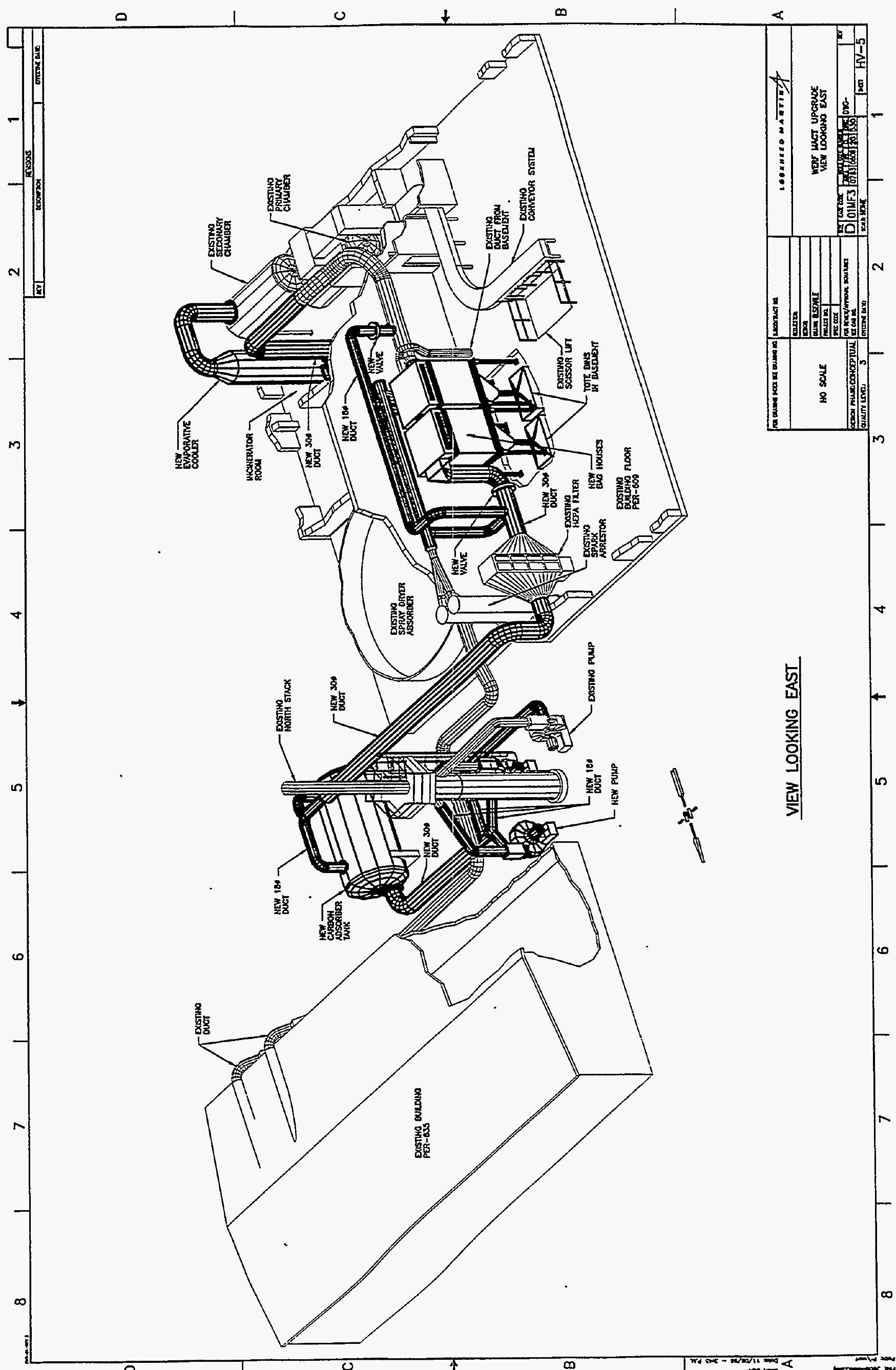




\section{APPENDIX D}

\section{ENGINEERING CALCULATIONS}




\section{WERF MACT Upgrade}

\section{ANALYSIS OF WERF STANDBY POWER SYSTEM CAPACITY TO SUPPORT LARGER INDUCED DRAFT FAN}

\section{Existing North Stack I.D. FAN (IOG-BLO-2)}

Rated horsepower $=60$ H.P.

Full load current $=77$ amps (Current the motor will draw when operating at 60 H.P.)

Running power $\quad=\mathrm{IV}$

$$
\begin{aligned}
& =(77 \mathrm{amps})(480 \mathrm{v}) \sqrt{3} \\
& =64,000 \mathrm{VA}=64 \mathrm{kVA}=64 \mathrm{~kW} \text { (unity power factor } \mathrm{kVA}=\mathrm{kW} \text { ) }
\end{aligned}
$$

\section{Existing WERF Standby Power System (Per EDF- 288 Rev. 1)}

Rated capacity $=225 \mathrm{~kW}$

Incinerator operation requires $143 \mathrm{~kW}$

Capacity available: $225 \mathrm{~kW}-143 \mathrm{~kW}=82 \mathrm{~kW}$

Additional amperage available where $I=\frac{P}{V}$

$I=\frac{82,000 \mathrm{VA}}{(480 \mathrm{v}) \sqrt{3}}=98$ amps available

\section{NEW NORTH STACK I.D. FAN}

Assumption: IOG-BLO-2 + new I.D. fan will not operate using Standby Power at the same time

Total current available to operate new fan: 77 amps +98 amps $=175$ amps

Fan Static Pressure Required: 50" w.c. vacuum

Required Flow: $12,000 \mathrm{acfm}$ at $250^{\circ} \mathrm{F}$

Assume

$\mathrm{Q}=8,000 \mathrm{acfm} / \mathrm{blower}$ (Only 6,000 acfm required per Kevin's M\&E Balance)

At $8,000 \mathrm{acfm} / \mathrm{fan}$, one (1) fan operating on standby power could be operated to gradually shut down incinerator operations.

Fan Power Requirements: 
Where:

$$
\begin{aligned}
& \mathrm{Q}=\text { Flow } \\
& \mathrm{FSP}=\text { Fan Static Pressure } \\
& \mathrm{VP}=\text { Velocity Pressure @ outlet of fan } \\
& \mathrm{CF}=6356 \text { (conversion factor) } \\
& \square=\text { efficiency at } 60 \% \text { (assumed) } \\
& P W R=\frac{(Q)(F S P+V P o u t l e t)}{(C F) \eta}
\end{aligned}
$$

Where:

$$
\rho=\quad \text { density of gas }=0.045 \frac{\mathrm{lbm}}{\mathrm{ft}^{3}} \text { (Density of air at } 250^{\circ} \mathrm{F} \text { will be close to actual offgas.) }
$$

$$
V P=\rho\left(\frac{\text { velocity }}{1096}\right)^{2}
$$

Where:

$$
\begin{aligned}
& V e l=\frac{Q(\text { flow through duct })}{A(\text { area of duct })} \\
& A=\frac{\pi \mathrm{d}^{2}}{4}=\frac{\pi 2^{2}}{4}=3.14 \mathrm{ft}^{2} \\
& V P=\left(0.045 \frac{\mathrm{lbm}}{\mathrm{ft}^{3}}\right)\left(\frac{2,548}{1096}\right)^{2}=0.24 \mathrm{in} .
\end{aligned}
$$$$
P W R=\frac{(8,000)(0.24+50)}{(6356)(.60)}=105 \text { horsepower (approx.) }
$$

105 H.P. $\times .7457 \frac{\mathrm{kW}}{H . P .}=78 \mathrm{~kW}$

$$
I=\frac{P}{V} \Rightarrow \frac{78,000}{480 \sqrt{3}}=94 \mathrm{amps}
$$

$\therefore$ For normal operating conditions, the standby power system can support a larger I.D. fan of approx. 100 H.P. 


\section{Startup of New North Stack I.D. Fan}

Although the WERF Standby Power System can supply the full load current associated with a 100 105 H.P. motor, there is a concern during fan startup. During startup, the fan motor will draw a large amount of current (in-rush current) to get started. The in-rush current varies among motors but can be as high as 5 times the full load current for short periods of time. The ability of the existing standby diesel generator to meet a larger motor's in-rush current demand is an unknown at this time. The use of an adjustable frequency controller to aid in the gradual startup of the fan is advised and may eliminate this concern.

\section{Engineering Calculation}

\section{Sizing Carbon Bed:}

Design Feature: Superficial Velocity $=50 \mathrm{ft} / \mathrm{min}$

Option 1:

Gas volumetric flow rate entering carbon bed $=10,500 \mathrm{ft}^{3} / \mathrm{min}$

Cross-sectional area $=\left(10,500 \mathrm{ft}^{3} / \mathrm{min}\right) /(50 \mathrm{ft} / \mathrm{min})=210 \mathrm{ft}^{2}$

According to vendor (NUCON International):

If $1.5 \mathrm{~mm}$ sulfur-impregnated pellets are used, bed depth of $2 \mathrm{ft}$ is adequate to achieve near greater than $99.9 \% \mathrm{Hg}$ removal. Actual quote = "nearly $100 \%$ removal"

Carbon pellet density: $35 \mathrm{lb} / \mathrm{ft}^{3}$

Total mass of carbon required $=\left(210 \mathrm{ft}^{2}\right)(2 \mathrm{ft})\left(35 \mathrm{lb} / \mathrm{ft}^{3}\right)=14,700 \mathrm{lb} \mathrm{C}$

Maximum $\mathrm{Hg}$ loading is $0.2 \mathrm{lb} \mathrm{Hg} / \mathrm{lb}$ pellets

Assume only $85 \%$ of carbon utilized before breakthrough occurs.

Maximum $\mathrm{Hg}$ capacity of $\mathrm{C}$ bed $=(14,700 \mathrm{lb} \mathrm{C})(0.2 \mathrm{lb} \mathrm{Hg} / \mathrm{lb} \mathrm{C})(0.85)=2,500 \mathrm{lb} \mathrm{Hg}$

Expected $\mathrm{Hg}$ loading during life of bed (10 years)

Hourly $\mathrm{Hg}$ feed rate $=10 \mathrm{~g} / \mathrm{hr}=0.022 \mathrm{lb} / \mathrm{hr}$

Hours of operation during one year $=5,760 \mathrm{hr}$

Total $\mathrm{Hg}$ loading $=(0.022 \mathrm{lb} / \mathrm{hr})(5,760 \mathrm{hr} / \mathrm{yr})(10 \mathrm{yr})=1,270 \mathrm{lb} \mathrm{Hg}$

Fraction of $\mathrm{C}$ bed utilized: $1,270 / 2,500=0.51$ 


\section{PERFORMANCE CURVE}

nị̄b | New York Bitower

ro oetermine performance

tt another RPM multiply.

$$
\begin{aligned}
& \text { 1. CFM }=K \\
& 2 \cdot S P \times K^{2} \\
& =-4 P
\end{aligned}
$$

where $K$ is new RPM divided

by RPM shown at right.
C84-138481

Customer EG \& G IDAHO, INC.

Togaing P.O. $284-138481$

Size 334 Type SERIES 45 GI

8880 CFM of 28 "SP of 8.84885 at $\frac{2487}{Z}$ RPM af 43.52 BHP

File No. Z-4795 Date 18-UUN-84

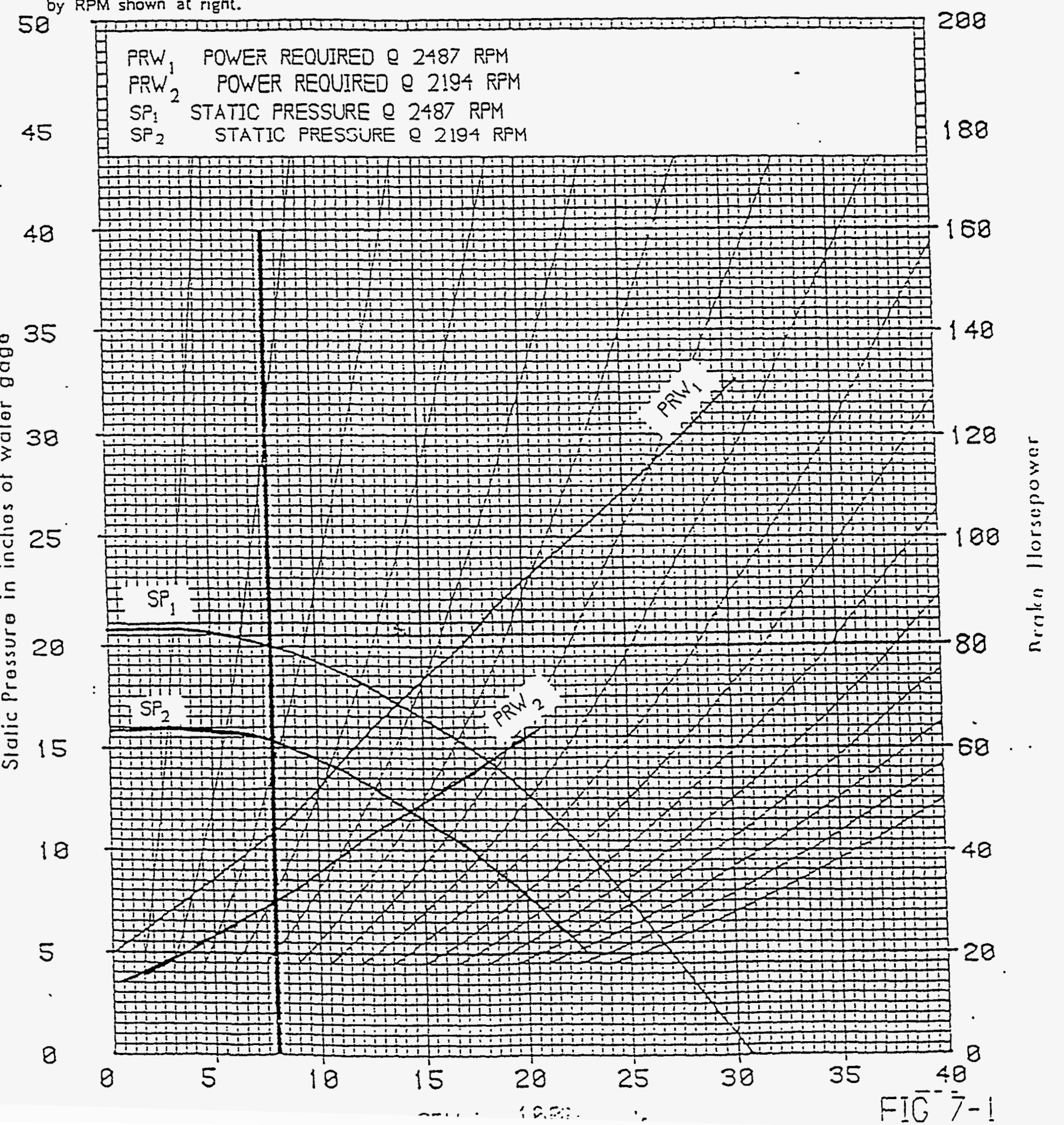




\section{PERFORMANCE CURVE}

nyD | New York. Bdower

To determine performanoe

at another RPM multiply:

$\therefore \cdot C F M \times K$
$2 \cdot S P=K^{2}$
$=$

where $K$ is new RPM divided

by RPM shown at right.
C84-138481

Customer's No.

Customer EG \& G IDAHD, INC.

Tagging P.D. $2084-130481$

Size 334 Type SERIES 45 GI

8888 CFM of 28 "SP of 8.84885

at 2487 RPM of 43.52 BHP

File No. Z-4785 Date 18-UUN-84

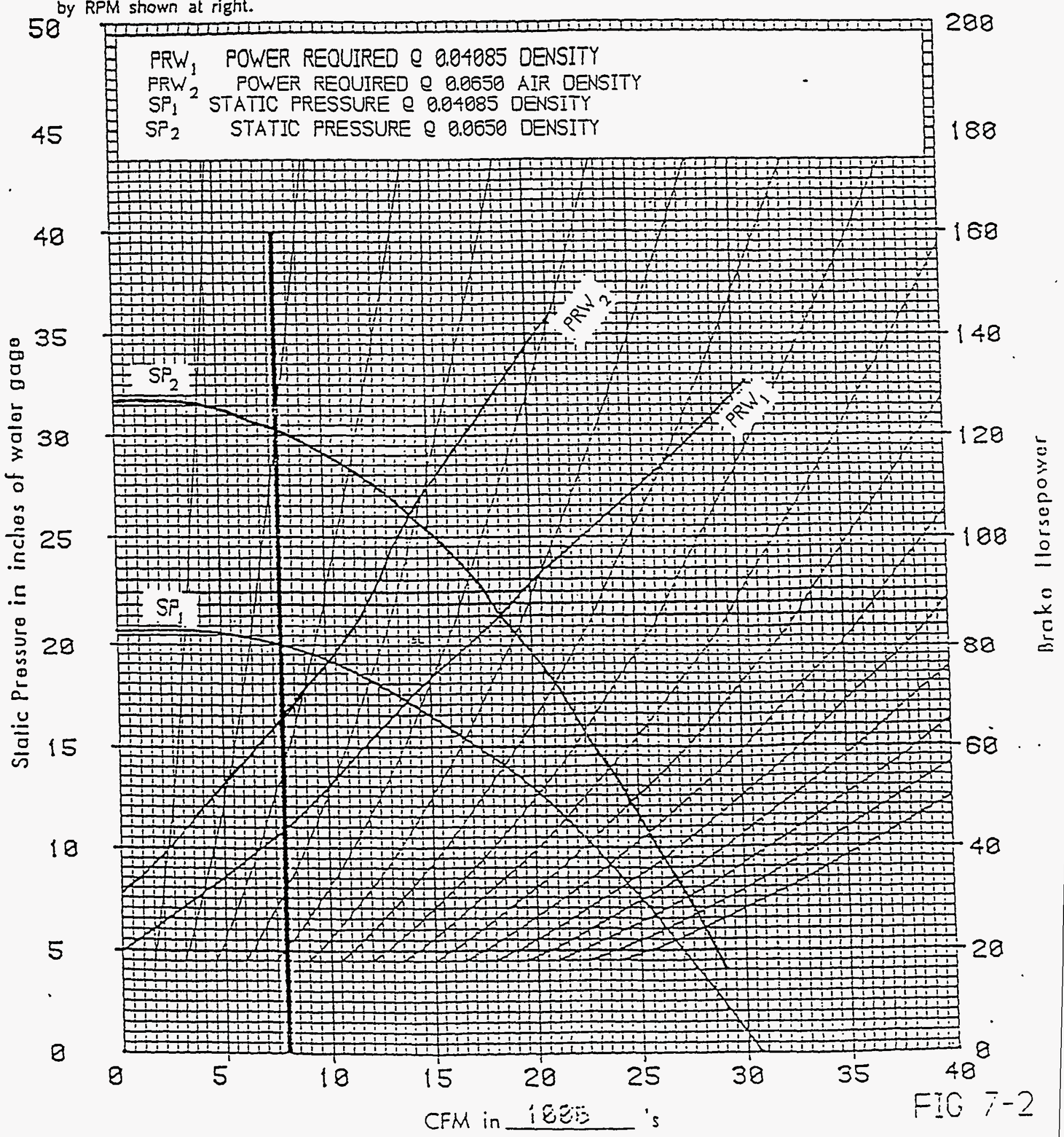


2

5) Ouilet gao flow

$$
\begin{aligned}
& Q_{\text {aur }}=Q_{1 N}+Q_{\substack{\text { air } \\
\text { Nozzic }}}+Q_{\text {sieam }} \text { where } Q_{\text {IN }}=2,125 \text { scfm } \\
& Q_{\text {nozzic }} \approx 25 \mathrm{scFm} \\
& \text { Qsram }=51 \frac{1 \mathrm{~ms}}{\min } \times \frac{21.8 \pi^{3}}{6 \mathrm{vi}}=1,357 \mathrm{sim}
\end{aligned}
$$

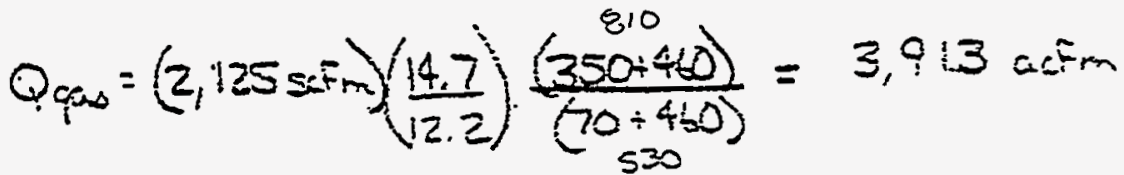

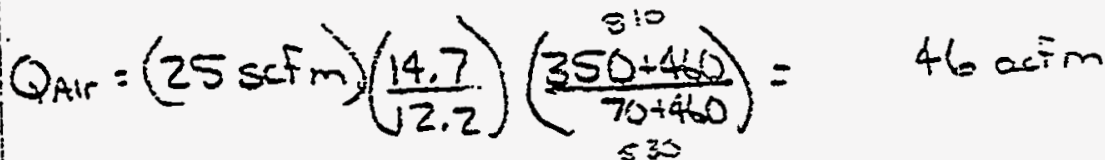

$$
\begin{aligned}
& Q_{\text {stam }}=1,367 \sin \frac{(14,7)}{(12,2)} \frac{(350+40)}{(212,400)}=1,9550<i m \\
& \therefore \text { Qout }=5,94 \text { actim } \\
& Q_{\text {in }}=11,40000 \mathrm{~m} \\
& Q_{\text {out }}=5,974 \text { axim } \quad Q_{\text {avg }}=\frac{11,400+5,944}{2}=8,6720 \mathrm{cFm}
\end{aligned}
$$

Aswnption: Velocity through cooler $700 \frac{\mathrm{ft}}{\mathrm{mind}}$ max par Reference

$$
\begin{aligned}
& V e t=\frac{Q_{\text {ave }}}{\text { Arca }} \\
& V=\frac{Q}{\frac{\pi D^{2}}{4}}=\frac{40}{\pi 0^{2}} \\
& D^{2}=\frac{4 Q}{Y \pi} \\
& D=\sqrt{\frac{4 Q}{V \pi}}=\sqrt{\frac{(4)(8,272)}{(700) \pi}}=3.97 \mathrm{FT} \approx 4 \mathrm{Ft} \\
& D=5 F T
\end{aligned}
$$

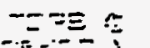




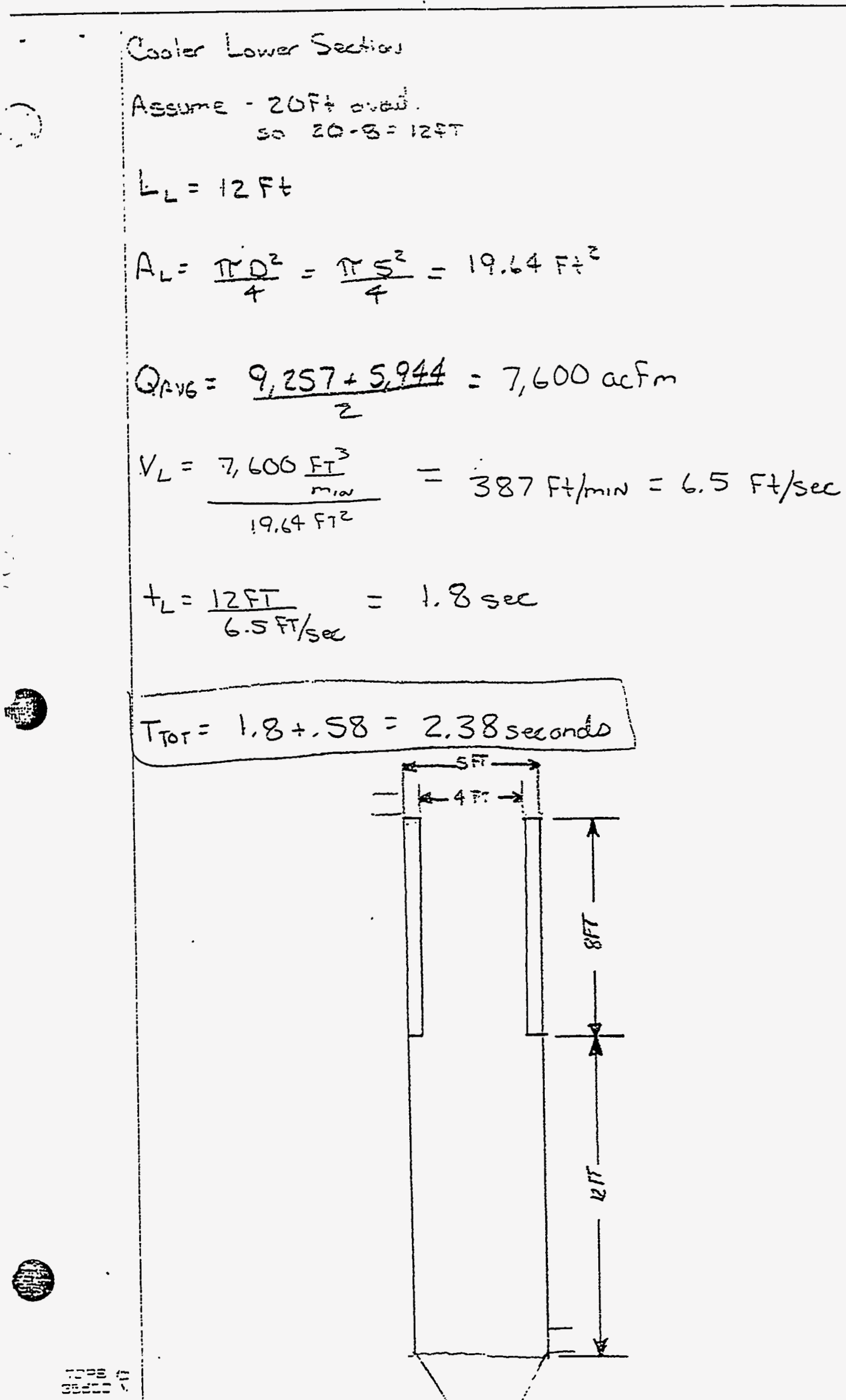




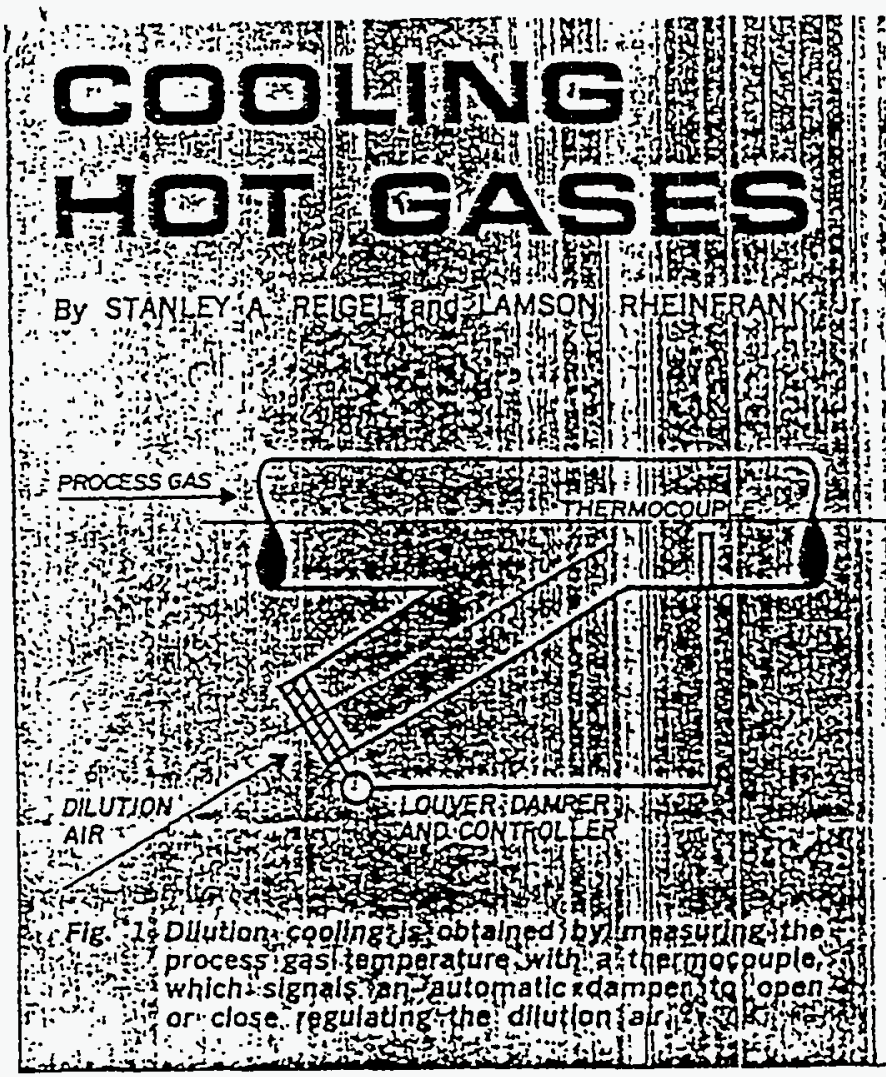

All air pollution control and duct systems have temperature limitations. Dry mechanical or wet collectors fabricated from mild steel are limited to approximately $1000 \mathrm{~F}$ inlet gas temperature. The maximum inlet gas temperature that can be toleraled by a baghouse depends upon the type of material used in the fabric tubes. Table I lists the maximum operating temperature of several common fabrics.

Elficiency of an electrostatic precipitator depends to a large extent, on the electrical resistivity of the dust. Between 200 and $500 \mathrm{~F}$. dust resistivity is at a maximum and difficult to electrically charge particles. It is advantageous, therefore, to operate electrostatic precipitalors at high temperatures. The apparent resistivity of dust can also be decreased by increasing the humidity of the carrier gas, although this effect is negligible at temperatures above about $600 \mathrm{~F}$. Evaporative coolers are used to reduce hot gas temperatures to approximately $700 \mathrm{~F}$ in order not to exceed construction material limitations.

The common methods used to cool a gas stream prior to entering an air pollution control device are dilution cooling, radiation/convection cooling, and evaporative cooling.

\section{Dilution Cooling}

Dilution cooling is simply adding ambient air to the hot process gas stream in sufficient quanlities 10 yield the desired mix lemperaiure. For computation purposes the ambient air is taken to be $100 \mathrm{~F}$ and the specific heat is taken at the arithmetic average of the maximum and minimum temperalures. A simple heal balance is

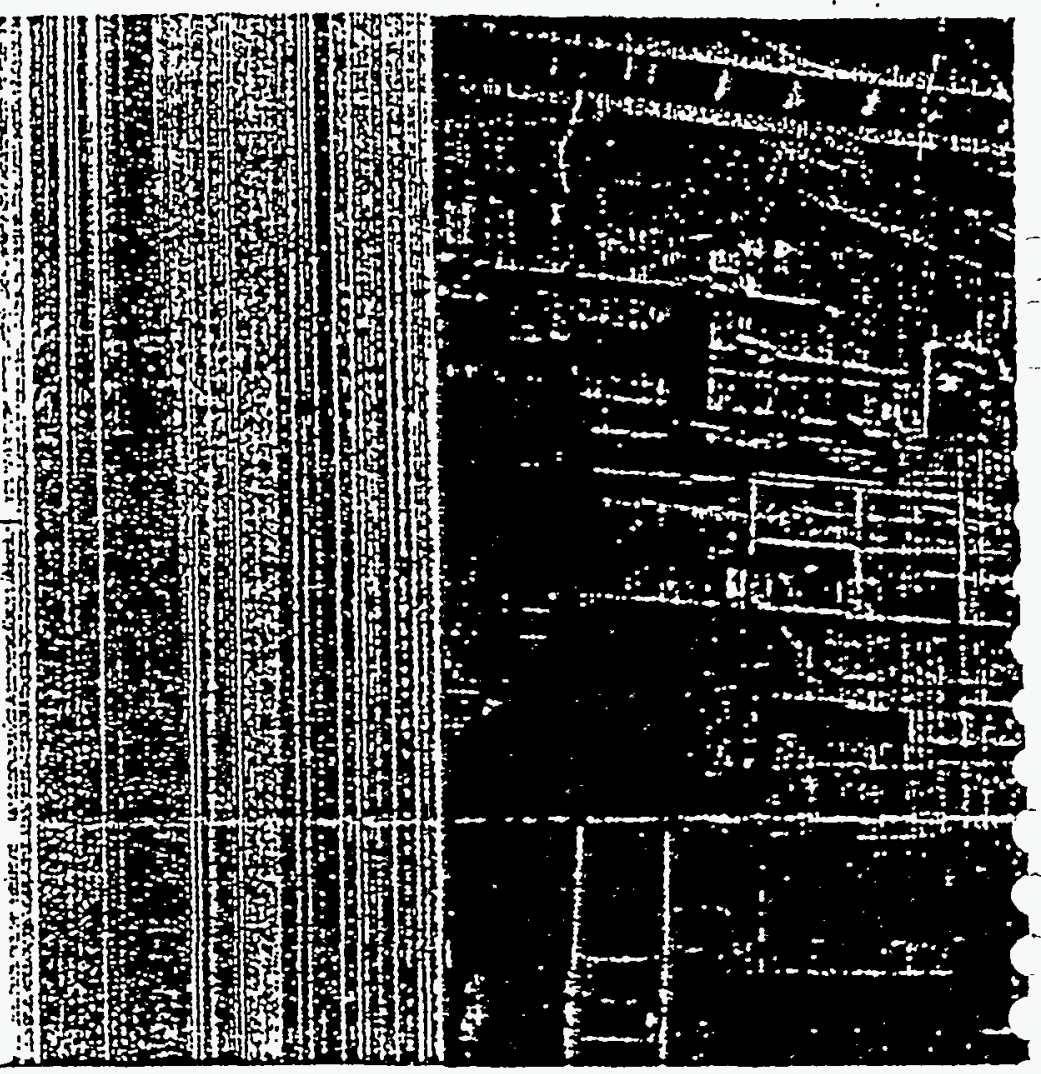

used when the specific humidity of the process gases is below $0.10 \mathrm{lb}$ of water per pound of dry air.

The primary disadvantage of dilution cooling is the increased volume of process gas which must be treated, and the corresponding increase in equipment and ductwork size downslream from the point of cooling. For example: $100,000 \mathrm{clm}$ of dry air at $1500 \mathrm{~F}$ is increased to $185,000 \mathrm{~cm}$ when cooled $10500 \mathrm{~F}$ by the addition of dilution air at $100 \mathrm{~F}$.

Although dilution cooling is not the best method for producing large temperature decreases, it is an excellent method for modulating temperatures about a lixed set point, Fig. 1. A tempering air bleed-in damper can be automatically modulated by a thermocouple in the downstream main duct.

\section{Radiation/Convection Cooling}

When a hol gas flows in a duct, heal is released to its surroundings by a combination of radiation and natural convection. The rate of heat transter can be calculated from the simple relatlon:

$$
\text { where } \begin{aligned}
Q= & U A \Delta T_{1} . \\
U= & \text { rate of heat transfer, BTU/hr } \\
& (h r)\left(f^{7}\right)(F) \\
A= & \text { Surface area of duct, } f^{2} \\
\Delta T_{1}= & \text { log-mean lemperature diflerence, } F
\end{aligned}
$$

Overall heat transfer coelficient is delermined empirically by correlating various dimensionless parameters such as the Reynolds number. The " $U$ " faclor for most hot gas applications will be in the range of from 1.5 to 3.0 BTU, (hr) $\left(f^{3}\right)(F)$. 


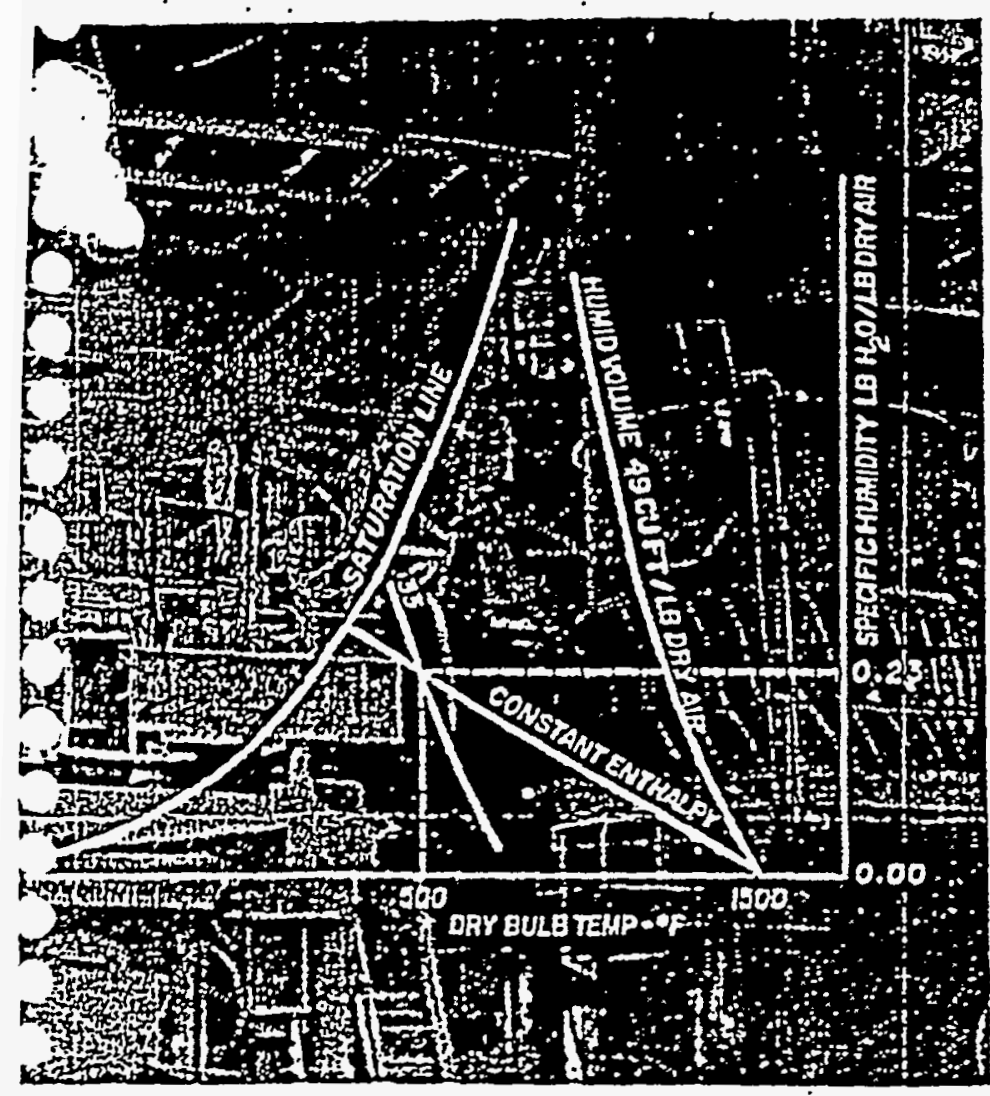

Fig. 2 Psychrometric chart.

Table. Operating temperatures of fabric bag materials.

\begin{tabular}{|c|c|c|}
\hline FABRIC & $\begin{array}{l}\text { Melting } \\
\text { Temp. }\end{array}$ & $\begin{array}{c}\text { Recommended } \\
\text { Maximum } \\
\text { Continuous } \\
\text { Operating } \\
\text { Temp. }\end{array}$ \\
\hline Cotton & $\begin{array}{l}\text { Decomposes } \\
\text { at } 302^{\circ} \mathrm{F}\end{array}$ & $180^{\circ} \mathrm{F}$ \\
\hline Wool ..- & $\begin{array}{c}\text { Chars at } \\
572^{\circ} \mathrm{F}\end{array}$ & $200^{\circ} \mathrm{F}$ \\
\hline Nylon 6, 6(1) & $480^{\circ} \mathrm{F}$ & $200^{\circ} \mathrm{F}$ \\
\hline Dacron( $\left.{ }^{2}\right)$ & $482^{\circ} \mathrm{F}$ & $275^{\circ} \mathrm{F}$ \\
\hline Nomex(') & $\begin{array}{c}\text { Decomposes } \\
\text { at } 700^{\circ} \mathrm{F}\end{array}$ & $350^{\circ} \mathrm{F}$ \\
\hline Orlon(') & $\begin{array}{l}482^{\circ} \mathrm{F} \\
\text { Softens }\end{array}$ & $260^{\circ} \mathrm{F}$ \\
\hline Creslan( $(7)$ & $\begin{array}{l}475^{\circ} \mathrm{F} \\
\text { Softens }\end{array}$ & $250^{\circ} \mathrm{F}$ \\
\hline Dynel(3) & $\begin{array}{l}325^{\circ} \mathrm{F} \\
\text { Softens }\end{array}$ & $160^{\circ} \mathrm{F}$ \\
\hline Polypropylene & $333^{\circ} \mathrm{F}$ & $200^{\circ} \mathrm{F}$ \\
\hline Teflon(') & $\begin{array}{l}\text { Decomposes } \\
\text { at } 750^{\circ} \mathrm{F}\end{array}$ & $\begin{array}{l}500^{\circ} \mathrm{F} \\
\text { emits toxic } \\
\text { gas at } \\
450^{\circ} \mathrm{F}\end{array}$ \\
\hline Fiberglas & $1470^{\circ} \mathrm{F}$ & $550^{\circ} \mathrm{F}$ \\
\hline Filtron(') & $\begin{array}{l}505^{\circ} \mathrm{F} \\
\text { Softens }\end{array}$ & $270^{\circ} \mathrm{F}$ \\
\hline
\end{tabular}

Dupont Reg. Trademark american Cyamamid Reg. Trade. mark "Union Carbide Reg. Trademark 'W. W. Cisswell Trade. name
Because of the relatively large surface area necessary to cool gas by radiation and convectlon, ductwork is usually arranged in looped_columns. To cool 100,000 cim of process gas from $1500 \mathrm{~F}$ to $500 \mathrm{~F}$, assuming $U=2.75 \mathrm{BTU} /(\mathrm{hr})\left(\mathrm{ft} \mathrm{f}^{2}\right)(\mathrm{F})$ at a gas velocity of $5000 \mathrm{fpm}$. would require more than $1000 \mathrm{ft}$ of 5 -ft-diameter duct. Assuming a fan efficiency of 70 per cent, 150 brake horsepower would be required to move the process gas through the radiation convection cooler. At $\$ 65$ per horsepower/year, the yearly operating cost would be approximately $\$ 10,000$ not including the cost of material removal from the cooler hoppers. This cost can be substantial whether done manually or by automated screw conveyors. Because of large space requirements, high pressure drop and lack of system llexibllity, radlation/convection cooling is generally not advantageous.

\section{Evaporative Cooling}

This method of cooling involves introducing water into a hot gas stream. The latent heat of vaporization of the water is supplied by the hot gas which results in a temperature decrease. Because the process is rapid it may be considered adiaballc. Calculations for evaporative coolers are simplified by the use of a psychrometric chart:

Example: Determine the water requirement and resultant volume flow rate for the evaporative cooling of $100,000 \mathrm{clm}$ of essentially dry alr from $1500 \mathrm{~F}$ to $500 \mathrm{~F}$.

Solution: By relerring to a psychrometric chart and the schematic chart. Fig. 2, find:

$\begin{array}{lll} & 1500 \mathrm{~F} & 500 \mathrm{~F} \\ \text { Humid volume (cu ft/lb dry air) } & 49 & 33 \\ \text { Specifle humldity (lb waler/lb dry alr) } & 0.00 & 0.23\end{array}$

$\mathrm{H}_{2} \mathrm{O}$ usage $=$

$100,000 \mathrm{cfm} \times \mathrm{lb} D \mathrm{~A} \times(0.23-0.00) \mathrm{lb}$ water $=56.5 \mathrm{gpm}$

$$
\overline{49 \mathrm{cuft}} \overline{\mathrm{lbDA} \times 8.3 \mathrm{lb}} . \ldots . .4 .
$$

Volume at $500 \mathrm{~F}=$

$$
100,000 \mathrm{cfm} \times \frac{33 \mathrm{cu} \mathrm{ft} / \mathrm{lb} D A}{49 \mathrm{cu} \mathrm{t} / / \mathrm{b} \mathrm{DA}}=67,500 \mathrm{clm}
$$

Low water consumption and total gas volume decrease make evaporative cooling attractive. The pressure drop across a well designed evaporative cooler should be no more then $2.5 \mathrm{in}$. w.g. at standard conditions (assuming 70 per cent fan elficlency) giving a 17.0 brake horsepower requirement for moving air. An additional 20 bhp would be required to pump the water (assuming 400-psi spray nozzles) for a tolal horsepower requirement of approximately 30 bhp. Assuming $\$ 65$ per horsepower-year, this amounts 10 an annual power cost of $\$ 1,950$. The cost of water for a year's operation would be approximately $\$ 3,000$ making the total annual operating cost approximalely $\$ 4,950$.

Evaporative cooler design should ensure that no water droplet carryover exists to foul dust collector surfaces. For this reason waler should be inlroduced as a fine spray or mist to evaporate all droplets belore the troated gas exits trom the cooler. A well designed 


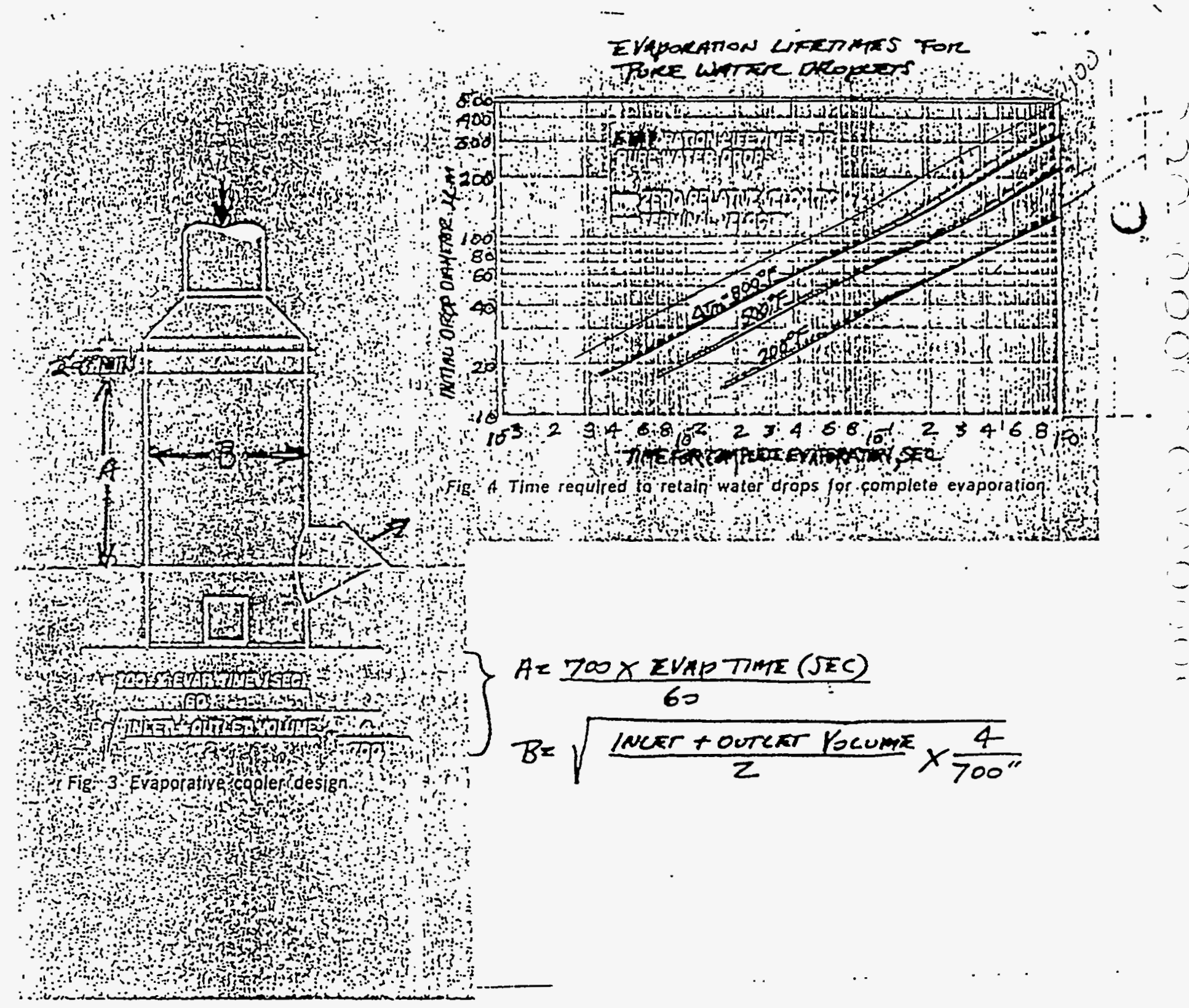

evaporative cooler is sized to provide the droplet retention time necessary for complete evaporation.

The diameter of an evaporative cooler is determined by limiting the gas velocity to $700 \mathrm{fpm}$ based on the average gas volume flow rate at the inlet and outlot. The effective height of the cooler is the distance between the last spray nozzle and the centerline of the outlet, Fig. 3.

The mean droplet size of hydraulic nozzles operatlng at relatively high pressures is $\mathbf{1 0 0 - 2 0 0 ~ m i c r o n . ~ I t ~ i s ~}$ important to provide sprays with small-dlameter water droplets because the retention time-and therelore the cooler size-vary directly as the square of the initial drop diameter. Consequently, the optimum spray for evaporative cooling of hot gases is a small uniform drop distribution.

Fig. 4 may be used to estimate the retention time for complete evaporation of water drops. For purposes of calculation, the mean temperature difference belween the drop and the surrounding air of $\Delta T_{N}$ may be taken as the average of the inlet and the desired outlet temperature of the spray cooler less the adiabatic salura-
Since rapld drop evaporation is enhanced by a uniform small drop distribution, the choice of spray nozzles is critical.

Evaporative cooling is a very effective means of reducing gas temperatures to pollution control systems at a low initial investment, low operating cost, and small space requirements. Reduction of gas temperature will normally permit use of less expensive pollution control systems.

For a copy of thals article circle BO4 on Reader Sorvica Card 


\section{Selecting and Applying Spray Nozzles}

By E. GRABACKI, Spraying Systems Co., Wheaton, IL

praying is used in most industri- al plants. Although the applications vary widely, there is one common denominator-the spray nozzle. The nozzle is a control device that emits a metered amount of liquid in droplet form in a controlled spray pattern.

The choice of a nozzle for a specific application depends on the function to be performed: cooling, coating, cleaning, humidifying, lubrication, dust control, fire protection, or gas scrubbing. For each application there is a nozzle with optimum performance characteristics. The quantity of liquid delivered and the spray pattern that are ideal for one application may be totally unsuited for another.

Important spray application requirements are spray pattern, capacity, spray coverage, particle size, and spray impact. These factors do not share equal importance in all applications. In each situation there is a specific value for each characteristic. Further, within each of these factors, there is a wide selection of choices.

Spray patterns include five basic types: solid stream, hollow cone, full cone, flat, and mist atomizing.

A solid-stream spray pattern is basically a uniform stream of liquid emitted through a drilled hole. However, modern solid-stream nozzles have been refined by use of proper inlet chamber proportions and contours ahead of the orifice or by addition of internal flow stabilizing vanes. These nozzles provide prolonged solid-stream integrity and delay the start of breakup and particle formation for a greal dis- tance from the nozzle, Fig. 1.

The hollow-cone spray pattern is essentially a circular ring of liquid. This pattern is generally formed by use of internal inlets that are tangential to a whirl chamber, or an internal grooved vane immediately upstream from the orifice. The whirling liquid results in a hollowcone configuration as it leaves the orifice, Fig. 2.

A full-cone spray pattern is usually round and completely filled with spray droplets. This spray pattern is normally formed by using an internal vane, which imparts controlled turbulence to the liquid ahead of the orifice, Fig. 3. Fullcone spray nozzles with square or oval spray patterns also are available.

A flat spray pattern distributes the liquid as a flat fan or sheet-type spray, Fig. 4. The flat spray pattern is formed by use of an elliptical orifice or deflector surfaces preceded by a round orifice.

In the elliptical orifice design, the axis of the spray pattern is a continuation of the axis of the inlet pipe connection.

In the deflector design, the defiection surface diverts the spray pattern away from the axis of the inlet pipe connection.

Straight-through elliptical orifice spray nozzles normally produce flat spray patterns with tapering edges. This characteristic is useful in establishing overlapping patterns between adjacent sprays on a multiple-nozzle header. The resulting distribution across the entire sprayed surface can therefore be uniform.

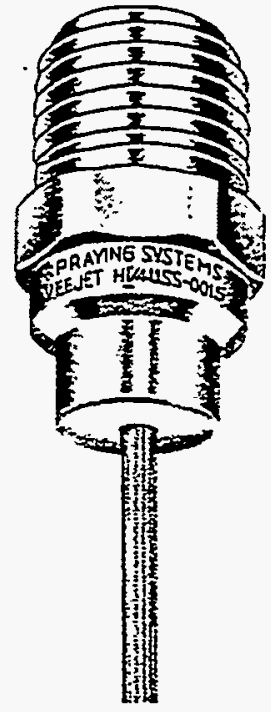

Fig. 1. Solid-stream spray pattern is projected for a great distance from the nozzle before it breaks up and particles form. Solid-stream and flat spray nozzles have higher velocity efficiency than fullcone and hollow-cone nozzles.

Fig. 2. Hollow-cone pattern resembles a funnel with liquid concentration in a circular ring. Section shows a tangential entrance to the whirlchamber.

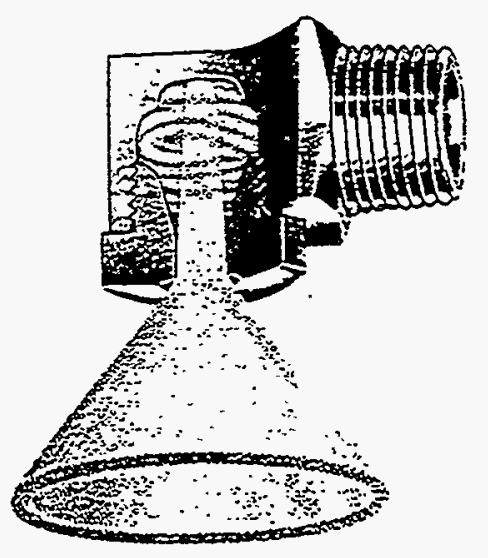


Flat spray nozzles with even spray distribution are usually used in cleaning applications that require uniform impact across the entire pattern width.

Mist spray patterns may be either hydraulic or air atomizing. These low-capacity nozzles produce a hollow-cone spray pattern, Fig. 5 . However, because the spray particles are very small, the spray pattern is affected by air friction and air currents, and it is not maintained for long distances. Several feet from the nozzle, depending on spraying pressure and nozzle capacity, the hollow-cone spray pattern disappears as the particles become suspended in air. This same general performance is provided by nozzles used for spray-drying applications at pressures of 1000 psi or greater.

Air-atomizing nozzles provide the finest degree of atomization for a given capacity and pressure, Fig. 6. They may be used to provide a choice of patterns: round, wideangle round, or flat. The spray pattern remains only as long as the velocity of the atomizing air is maintained. When velocity decreases, the spray particles eventually may evaporate completely, depending on their size, exposure time, relative humidity, and other ambient conditions.

Capacities of standard commercial spray nozzles range from 0.1 gph to $10,000 \mathrm{gpm}$. At the low end of this range, compressed air is used to atomize the liquid spray, which is either siphoned into the nozzle or supplied under moderate pressure. Larger air-atomizing nozzles with capacities to $30 \mathrm{gpm}$ also are available. Capacities of standard hydraulic spray nozzles begin at approximately 1 gph and range to a maximum of $10,000 \mathrm{gpm}$. Higher capacities require custom-designed nozzles. Discharge capacity can be varied by changing the pressure differential of the liquid through the spray nozzle.

Capacities of specific, flat and hollow-cone spray nozzles are proportional to the square root of the pressure. For full-cone spray nozzles, the capacity-pressure relationship is similar, but not exactly the same. The liquid capacity is controlled only by the liquid pressure. Atomizing nozzles with internal mixing of air have a wide range of capacities which are controlled by the combination. of air and liquid pressures used.

Spray coverage can vary within each of the general classes of spray nozzles described. Nozzles with narrow, medium, or wide-angle spray coverages can be selected for the requirements of a specific application. Spray angles range between 5 and $180 \mathrm{deg}$, depending on the nozzle type. The notation spray angle is used as a quick reference to indicate the general nature of the spray coverage (see table).

Specifically, the spray-angle reading provided in manufacturers' catalogs is usually measured with a calibrated adjustable protractor, at approximately $1 \mathrm{ft}$ from the nozzle orifice, and at a specific pressure. Actually, as the spray continues past the I ft distance, the velocity generally decreases and the direction of spray particle travel is affected by gravity and air friction, thereby decreasing the effective spray angle. The net effect causes the spray to cover less area than indicated by published spray-angle figures. Spraying pressure also frequently affects the spray angle and the resulting spray coverage. When extremely narrow spray coverage is desired or when maximum impact is required, solid-stream nozzles can be used; these nozzles have a zero degree spray angle.
Fig. 3. Full-cone pattern is usually round, although square and oval pattern are also available. An internal vane imparts controlled turbulence to the liquid before it reaches the orifice to disperse droplets uniformly.
Fig. 4. Flat spray pattern is formed by use of an elliptical orifice or deflector surfaces preceded by a round orifice. Straight-through elliptical orifice nozzles can be used to produce a pattern with tapering edges.
Fig. 5. Mist spray patterns are formed initially in a hollow cone; however, because the particles are very small, the pattern is not maintained for long distances, because it is slowed by air friction and affected by air currents.
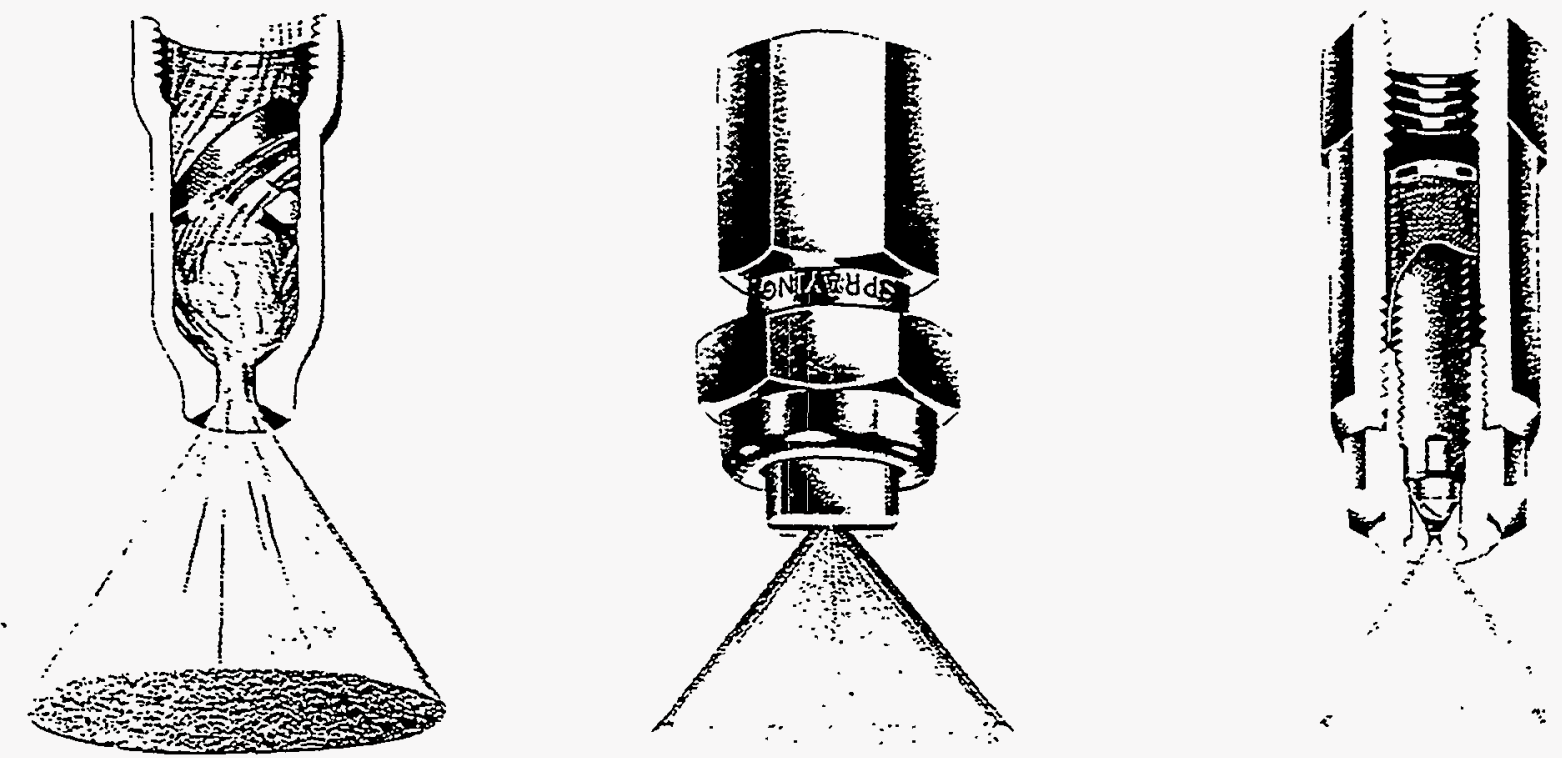
When the application requires an accurate measure of the spray coverage, the nozzle manufacturer should be consulted for information on the actual spray coverages obtained at varying distances from the nozzle-within a specified pressure range.

Particle size varies over a relatively wide spectrum; it is controlled by the nozzle type, size, and spraying pressure. The unit most commonly used to describe spray particle size is the median volume diameter (MVD) or the median mass diameter (MMD). By definition, 50 percent of the total volume of liquid sprayed consists of drops larger than the MVD, and 50 percent of the total volume consists of drops smaller than the MVD.

The MVD is usually expressed in microns $(1 / 25,400$ in. or 0.001 $\mathrm{mm})$. For example, wet fog particles are in the 10 to 15 micron range, light rain is in the 100 to 500 micron range, and the heaviest rainfall has particles varying from 2000 to 5000 microns in size. A typical flat spray nozzle, spraying 1 gpm at 40 psi, produces particles with an MVD of approximately 600 microns. Under similar conditions, the hollow-cone spray nozzle particle size is approximately $400 \mathrm{mi}$ crons, and a full-cone nozzle with an equivalent flow rate produces particles with an MVD of approximately 1600 microns.

Generally, for hydraulic spray nozzles, hollow-cone nozzles produce the smallest particle size ranges for a given capacity and pressure, followed by the fiat spray nozzles. The largest particles are emitted by equivalent full-cone spray nozzles. Very small particles are provided by air-atomizing nozzles that use compressed air for atomizing the liquid.

For a specific spray nozzle, the particle size is decreased by increasing the spraying pressure. This diminishing particle size relationship is more pronounced in the lower pressure ranges; there is less significant change at higher pressures.

It also follows that smaller particle sizes are produced by lowcapacity nozzles spraying at high pressures: and conversely, larger particles are produced by largecapacity nozzles sprayino ai low

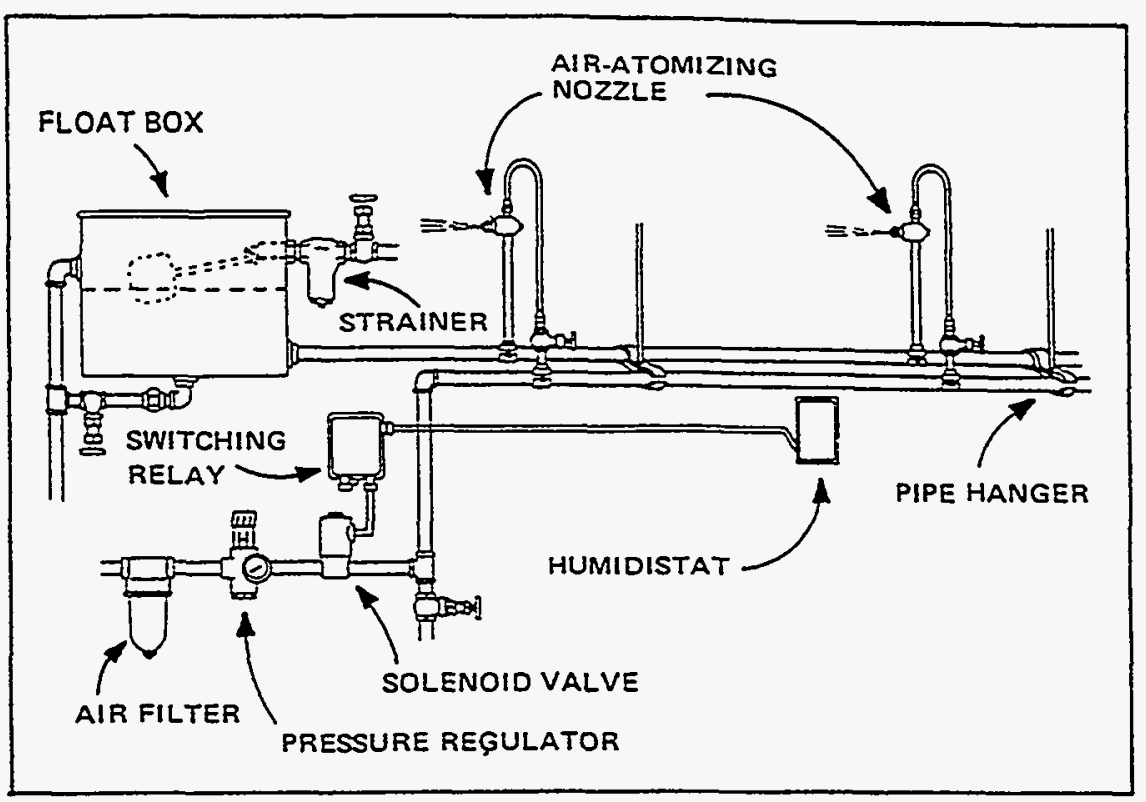

Fig. 6. Air-atomizing nozzles are used for humidifying, giving the finest degree of atomization for a given capacity and pressure. The spray patterns are maintained only as long as the velocity of the atomizing air can be maintained; spray particles usually evaporate within $15 \mathrm{ft}$ of the nozzles. The components required for a typical humidifying system are shown, with a humidistat for automatic control.

\begin{tabular}{|c|c|c|c|c|c|c|c|c|c|c|c|c|}
\hline & & & & $\begin{array}{l}\text { COVE } \\
\text { FOR V }\end{array}$ & $\begin{array}{l}\text { ZAGE } \\
\text { ARIO }\end{array}$ & $\begin{array}{l}\text { OF IM } \\
\text { S SPF }\end{array}$ & $\begin{array}{l}A C T \\
\text { AY An }\end{array}$ & $\begin{array}{l}\text { REA } \\
\text { GLES }\end{array}$ & & $=$ & & \\
\hline$\underset{\text { THEO }}{\text { COV }}$ & $\overrightarrow{A G A L}$ & & & & & & & & & & & \\
\hline $\begin{array}{l}\text { Spray } \\
\text { angle, }\end{array}$ & & Theor & ical c & verage & in., w & en the & distan & e from & nozzle & orifice & in., is & \\
\hline & 2 & 4 & 6 & 8 & 10 & 12 & 15 & 18 & 24 & 30 & 36 & 48 \\
\hline 5 & 0.2 & 0.4 & 0.5 & 0.7 & 0.9 & 1.1 & 1.3 & 1.6 & 2.1 & 2.6 & 3.1 & $\overline{4.2}$ \\
\hline 10 & 0.4 & 0.7 & 1.1 & 1.4 & 1.8 & 2.1 & 2.6 & 3.1 & 4.2 & 5.2 & 6.3 & 8.4 \\
\hline 15 & 0.5 & 1.1 & 1.6 & 2.1 & 2.6 & 3.2 & 3.9 & 4.7 & 6.3 & 7.9 & 9.5 & 12.6 \\
\hline 20 & 0.7 & 1.4 & 2.1 & 2.8 & 3.5 & 4.2 & 5.3 & 6.4 & 8.5 & 10.6 & 12.7 & 16.9 \\
\hline 25 & 0.9 & 1.8 & 2.7 & 3.5 & 4.4 & 5.3 & 6.6 & 8.0 & 10.6 & 13.3 & 15.9 & 21.2 \\
\hline 30 & 1.1 & 2.1 & 3.2 & 4.3 & 5.4 & 6.4 & 8.1 & 9.7 & 12.8 & 16.1 & 19.3 & 25.7 \\
\hline 40 & 1.5 & 2.9 & 4.4 & 5.8 & 7.3 & 8.7 & 10.9 & 13.1 & 17.5 & 21.8 & 26.2 & 34.9 \\
\hline 50 & 1.9 & 3.7 & 5.6 & 7.5 & 9.3 & 11.2 & 14.0 & 16.8 & 22.4 & 28.0 & 33.6 & 44.8 \\
\hline 60 & 2.3 & 4.6 & 6.9 & 9.2 & 11.5 & 13.8 & 17.3 & 20.6 & 27.7 & 34.6 & 41.6 & 55.4 \\
\hline 70 & 2.8 & 5.6 & 8.4 & 11.2 & 14.0 & 16.8 & 21.0 & 25.2 & 33.6 & 42.0 & 50.4 & 67.2 \\
\hline 85 & 3.7 & 7.3 & 11.0 & 14.7 & 18.3 & 22.0 & 27.5 & 33.0 & 44.0 & 55.0 & 66.0 & 88.0 \\
\hline 100 & 4.8 & 9.5 & 14.3 & 19.1 & 23.8 & 28.6 & 35.8 & 43.0 & 57.2 & 71.6 & 85.9 & 114 \\
\hline 120 & 6.9 & 13.9 & 20.8 & 27.7 & 34.6 & 41.6 & 52.0 & 62.4 & 83.2 & 104 & & \\
\hline 140 & 10.9 & 21.9 & 32.9 & 43.8 & 54.8 & 65.7 & 82.2 & 98.6 & & & & \\
\hline 160 & 22.7 & 45.4 & 68.0 & 90.6 & 113 & & & & & & & \\
\hline
\end{tabular}

pressures. The values for particle sizes vary with each nozzle, and are obtained by actual counts and measurements of spray particles. The techniques for measuring and counting particles vary, but the more sophisticated and more accurate techniques are based on counting and measuring particles in the spray pattern under actual spraying conditions.
Spray impact (total) is theoretically equal to $0.0526 Q \sqrt{P}$, where $Q$ $=$ flow in gpm and $P=$ pressure differential in psi. Therefore, impact can be increased by three methods: higher pressures to produce higher flow rates for a given nozzle, larger-capacity nozzles at the same pressure. or larger-capacity nozzles at higher pressures.

Alihcugh the total impace is im- 
portant, the impact per unit area is usually the more important consideration. For a given spray nozzle capacity and pressure, the narrowest available spray angle produces the highest impact per unit area. Frequently, as in cleaning applications, flat spray nozzles with nominal spray angles of 25 to $50 \mathrm{deg}$, spraying at high pressure, provide a reasonable compromise between the number of nozzles required and the impact requirement.

Impact is also enhanced by placing the nozzle close to the sprayed surface, which increases the volume of liquid per unit area of surface.

Other variables affect spray performance. Changing one variable in the spray control usually produces changes in other spray characteristics. For instance, with small-diameter orifice nozzles, the pressure may be increased until there is a detrimental effect on impact. Increasing the pressure results in finer spray atomization. These smaller particles are slowed by the air resistance and the impact force at the sprayed surface decreases. Therefore, higher pressures do not always produce better impact.

Most spray nozzles project a spray pattern on the same axis as the inlet pipe connection, although nozzle configurations with the spray pattern axis at 90 deg to the center line of the inlet connection also are ayailable. Another spray nozzle design is the wall-mounted type that permits attaching the orifice end of the nozzle to a threaded connection. This nozzle can be used to spray into a tank, while the nozzle inlet connection remains outside the tank.

Standard commercial nozzles are usually available in brass, cast iron, steel, stainless steel, polyvinyl chloride, polypropylene, and nylon. To meet specific corrosion and erosion problems, these same nozzles can also be supplied in special materials, including special stainless steels, exotic metals, other plastics, ceramics, and silicon carbide, or with carbide inserts. Nozzles of special materials are normally custom made.
Each nozzle series has à maximum operating pressure, either for spray performance or because of personnel or equipment safety considerations. This pressure depends on nozzle design, construction materials, and conditions of operation. For example, most brass nozzles are recommended for pressures of 125 psi or less, but nozzles used in spray-drying applications are recommended for pressures to 7000 psi. At the other pressure extreme, solid-stream nozzles are used in jet cutting applications at pressures to 60,000 psi.

Viscosity and specific gravity of the liquid affect nozzle performance. Published data on spray nozzle performance are usually based on spraying water. If a liquid has a viscosity-or specific gravity-significantly different from that of water, allowance must: be made for the influence of these characteristics on spray performance.

Liquid viscosity is a primary factor affecting spray pattern formation and, to a lesser degree, capacity. High-viscosity liquids require a higher minimum pressure to start formation of a spray pattern. And the spray angle is generally narrower throughout the pressure range than it is when water is sprayed.

The infiuence of increased viscosity varies with each nozzle design and is best determined by actual tests rather than by theoretical formulas. Tests indicate that full-cone spray patterns are most drastically influenced by viscous liquids, followed by fiat spray patterns; hollow-cone spray patterns are more adaptable for spraying viscous fluids. The higher the viscosity, the greater the problem of obtaining an acceptable spray pattern becomes. Increasing the spraying pressure helps overcome this problem, but frequentiy air atomization, or extremely high pressure. must be used to obtain any spray pattern at all. Using higher-capacity nozzles occasionally helps some viscosity-related problems.

Specific gravity of the fluid being sprayed has a basic effect on capac- ity. The capacity of a nozzle spraying a fluid having a specific gravity other than 1.0 is inversely proportional to the square root of the specific gravity. This specific gravity factor can then be applied to adjust catalog tabulations, which are usually based on water.

Selecting a nozzle for a spray application begins with establishment of the priority for a specific performance characteristic. The primary characteristic required to bring about the desired results must be identified. For instance, in cleaning operations, impact is the most important spray characteristic.

In gas cooling, spray particle size is the most important consideration.

Washing photographic film, on the other hand, requires a lowimpact spray.

In spray pond cooling applications, a medium-size spray particle is required; it can be produced by a relatively low spraying pressure. The low pressure limits drift and yet provides particle sizes with a reasonable ratio of water droplet surface area to mass of liquid.

In steel mill descaling operations, extremely high impact is required, while in continuous casting applications a uniform spray distribution pattern is the top priority.

In fire protection applications, nozzles are required to cover a specific area at a proper coverage rate.

In spraying additives in chemical mixing operations, small-capacity nozzles with uniform distribution characteristics are required.

These are but a few of many spray applications, each of which must be studied to determine the spray characteristics required so the nozzle that most closely meets total performance requirements can be selected.

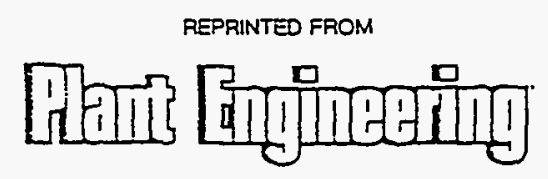

SEPTEMBER 161982

The magazine that helps plant engineers solve their everyday problems 
Appendix E SUPPORTING LITERATURE 


\author{
MACT Feasibility Study \\ Preliminary Process Hazards Analysis \\ October 21, 1998 \\ WERF Conference Room 9:30a.m.
}

The following individuals participated in the Preliminary Process Hazards Analysis for WERF's MACT Feasibility Study:

\begin{tabular}{ll} 
NAME & ORGANIZATION \\
\hline Bruce Bonnema & ER \\
Dave Moser & WROC/WERF \\
Charles Barnes & AEDL \\
Jack Prendergast & AEDL \\
Steve Poling & SAIC \\
Kevin Liekhus & AEDL \\
Shannon Corrigan & WROC \\
Al Kranning & WROC \\
Curtis Branter & WROC \\
Bob Shilkett & WROC \\
Roger Draper & SITE SERVICES \\
Dennis Conley & WERF
\end{tabular}

\author{
JOB TITLE/DUTIES \\ Consulting Eng./Design Lead for MACT \\ Feasibility Study \\ WERF Repack/Burn Plans \\ Process Engineer \\ Process \& Chemical Engineer \\ Mechanical Eng./MACT Study \\ Staff Engineer \\ WERF Incinerator Systems Engineer \\ WERF Electrical Systems Engineer \\ WROC Engineering Supervisor \\ Engineer/Varied \\ Drafting \\ Offgas Systems Engineer
}

Bruce Bonnema opened the meeting with a presentation of the WERF-MACT background and the proposed solution: to retrofit an evaporative cooler, dry sorbent injection and a carbon adsorber into WERF's Air Pollution Control System. Cost for this commercially proven technology is less than $\$ 5$ million, and will expand the WERF WAC for $\mathrm{Cl}$ and $\mathrm{Hg}$, as well as minimize dioxin and furan formation. Treatment of possible PCBs was mentioned, but this issue is not being addressed yet.

The objective of the meeting was to identify process hazards for the proposed MACT-compliant Air Pollution Control (APC) system that could release toxic or hazardous materials. Another objective was to identify design criteria or alternatives that could eliminate or reduce the process hazards. Early identification of potential hazards can be mitigated at minimal cost and disruption, and this will help the design team develop safety-related operating guidelines for the new APCS.

The review team conducted a Preliminary Process Hazards Analysis (PPHA) of the equipment using engineering flow diagrams. Steve Poling also presented a proposed equipment layout, as well as a P\&I diagram from another project completed by the supplier Bundy. Based on these design drawings, the team suggested process deviations that could occur. For each deviation that resulted in potential safety or hazardous material release consequences, the team reviewed safeguards (e.g., design features, safety equipment, and procedures) that protect against that deviation and/or its consequences. Action items were proposed for further evaluation if the existing protection was considered inadequate or if there was insufficient design information.

The action items resulting from the PPHA are shown on the attached tables. Most of the problems are design-related; some plant procedures may also need to be developed.

Cc: Mike Wolters

Kevin Kooda

Project File 


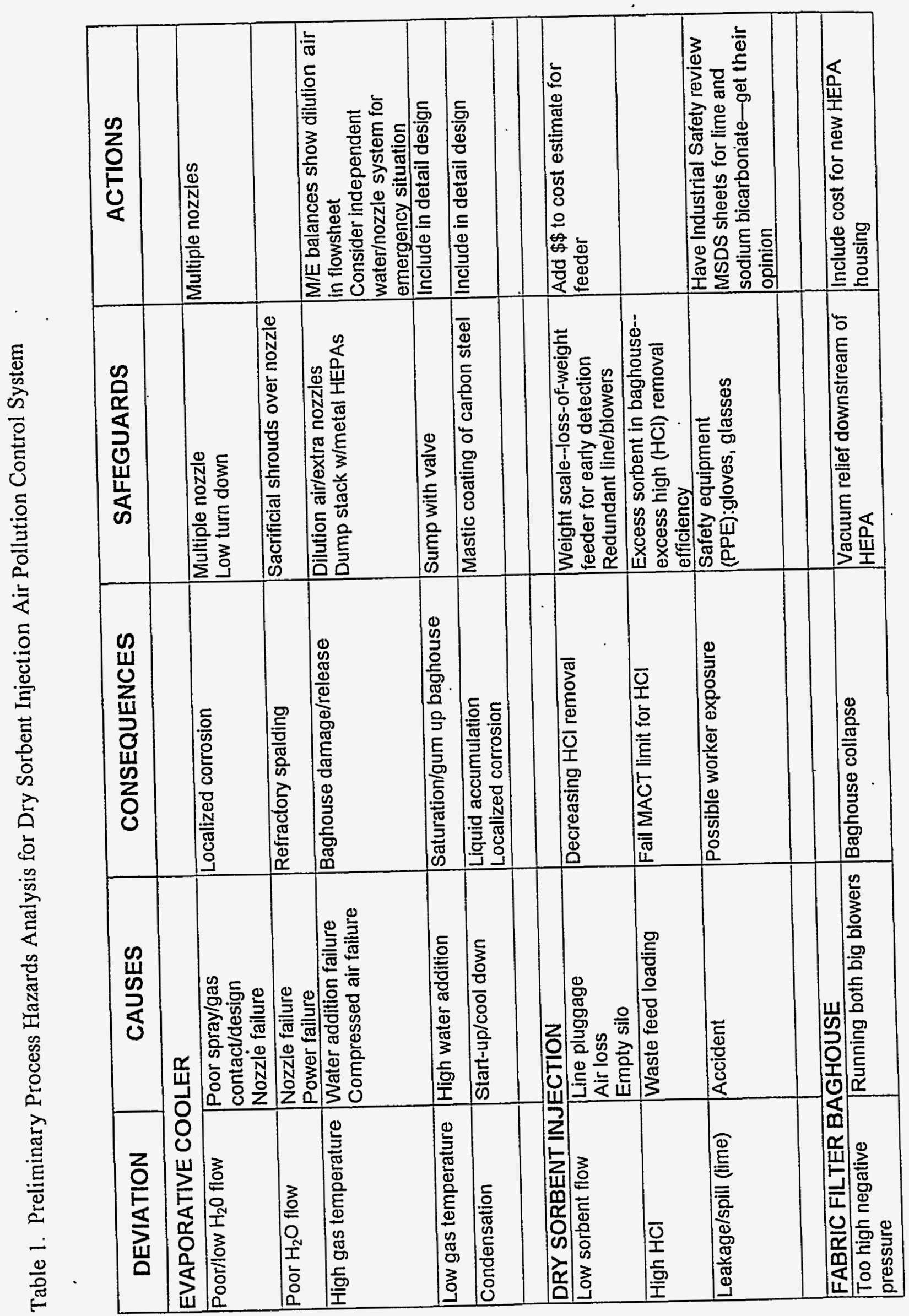




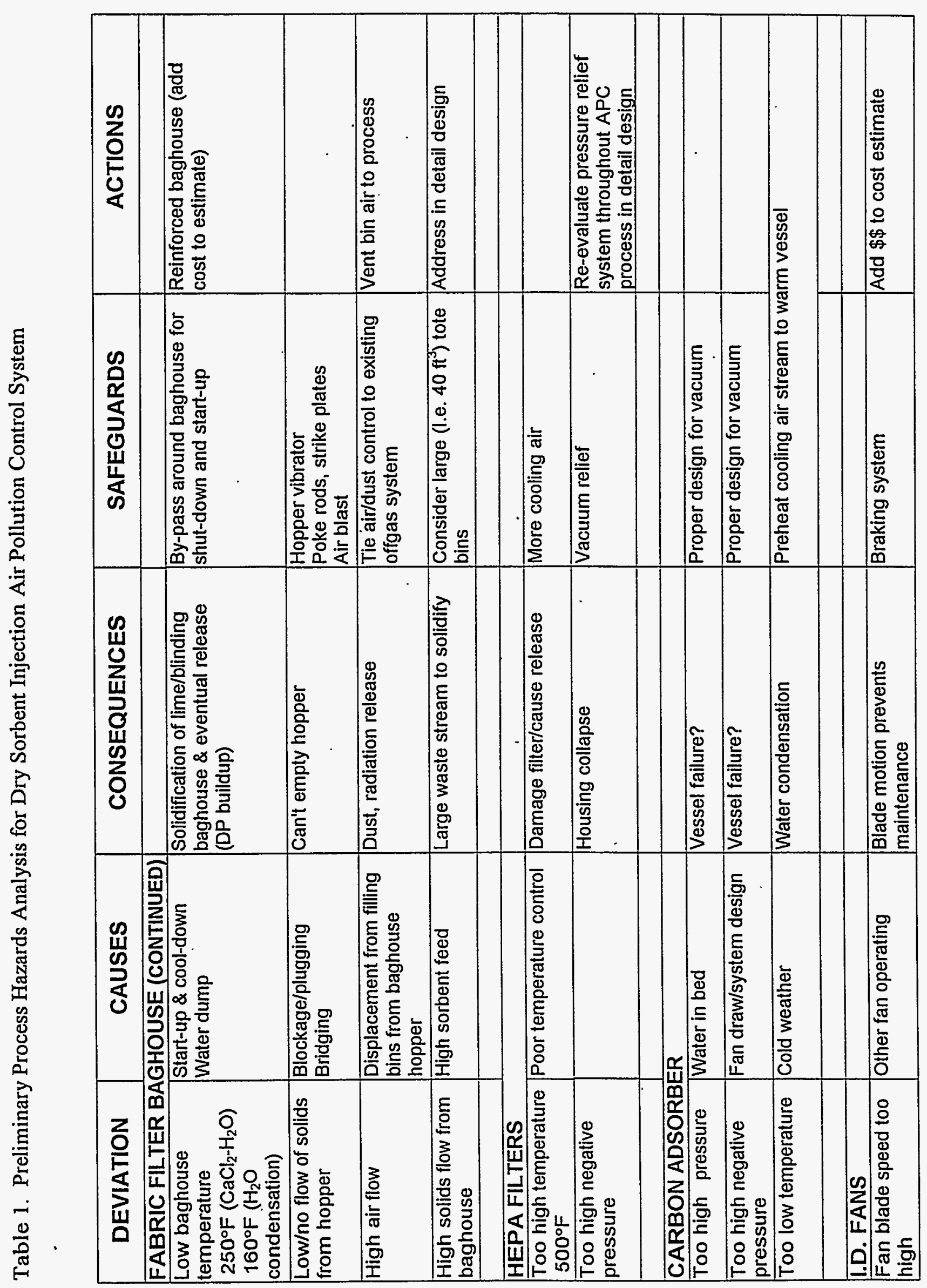




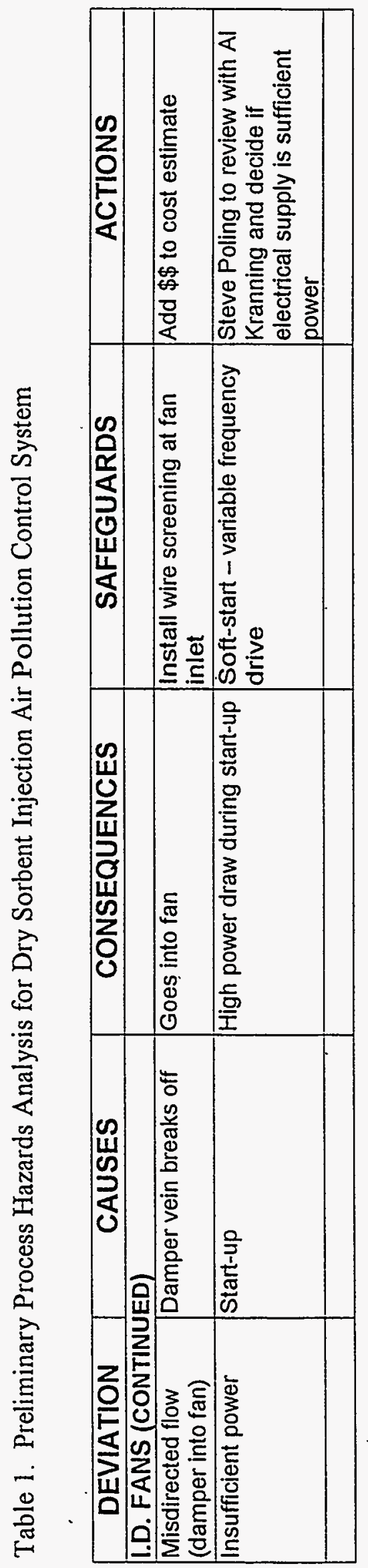




\section{RECORD OF TELEPHONE CONVERSATION}

Date: $9 / 14 / 98$

Person Called: Louis Benson

Company: Dravo Lime

Phone No.: (412) 995-5500

\author{
Person Calling: Kevin Liekhus
}

Background: Dravo markets a product called SORBALIT for use in a dry injection system upstream of a baghouse. The product contains hydrated lime for $\mathrm{HCl}$ removal, activated carbon for dioxins, and sulfur-impregnated carbon for $\mathrm{Hg}$ removal.

Conversation: Mr. Benson stated that $95 \% \mathrm{HCl}$ removal using hydrated lime are reasonable operating conditions in medical waste incinerators with no additional water added to the system. In a soil treatment facility, $99.5 \%$ removal efficiency was achieved for a gas stream with $50 \%$ water at $350 \mathrm{~F}, 35 \mathrm{lb} / \mathrm{hr} \mathrm{Cl}, 45,000 \mathrm{acfm}$ at $275 \mathrm{~F}$. Mr. Benson did not know the air-to-cloth ratio right offhand. When the facility operated the baghouse at only $1 \mathrm{in}$. w.g. pressure drop, only $70 \%$ removal was observed. When the pressure drop was allowed to increase to 4 in. w.g., the higher efficiency (99.5\%) was achieved. $\mathrm{He}$ did mention that the lime is added at approximately a 3:1 stoichiometric ratio. $\mathrm{He}$ said that facilities in the U.S. generally don't recycle material, although it is more common in Europe.

I asked Mr. Benson to help identify a facility (like the example above) for us to talk to or possibly visit. The fact that Mr. Benson was willing to end our phone conversation without asking for my phone number suggests he lacks the sufficient enthusiasm required to complete this task. I was able to leave my phone number with him, just in case.

He did note that there are some handling issues regarding hydrated lime. There is a risk the material will become packed within a container which can not be freed by vibration or screw conveyor. Since it is a low density material, it can bypass screw even when screw is turned off.

Sulfur-impregnated carbon (injected as part of SORBALIT mixture) has achieved 85$90 \% \mathrm{Hg}$ removal efficiency in baghouse operated at $285 \mathrm{~F}$.

My comments: A high-moisture offgas stream (our design condition is $35-40 \%$ moisture) and low air-to-cloth ratio appears to favor high efficiencies. The model shows for the conditions assumed for design calculations, $97.2 \%$ removal is required for $20 \mathrm{lb} / \mathrm{hr}$ $\mathrm{Cl}$ in feed to be discharged at $75 \mathrm{ppmv}\left(\mathrm{dry}, 7 \% \mathrm{O}_{2}\right)$. A feed rate of $10 \mathrm{lb} / \mathrm{hr} \mathrm{Cl}$ requires a removal efficiency of $94.4 \%$ to be discharged at $75 \mathrm{ppmv}\left(\mathrm{dry}, 7 \% \mathrm{O}_{2}\right)$. 


\section{RECORD OF TELEPHONE CONVERSATION}

\section{Date: 9-15-98}

Person Called: Jim Gaspar

Person Calling: Kevin Liekhus

Company: BFI Waste Systems, Inc., Houston, TX

Phone No.: (281) 870-7026

I spoke with Mr. Gaspar because BFI operates a dozen medical waste incinerators. I told him of our interest in visiting a facility that uses a dry lime injection process in conjunction with a baghouse. Two facilities were identified. One facility is in Baltimore and another near Orlando. The facility in Apopka, Florida was identified as being the best facility to visit because there is also a total quench evaporative cooler upstream of the baghouse. The Apopka facility burns $1900 \mathrm{lb} / \mathrm{hr}$.

The BFI facility in North Salt Lake City uses bicarbonate in dry sorbent injection system but the process does not use a baghouse. They use electrostatic precipitators (ESPs) and a wet scrubber to remove particulates and sorbent. 


\title{
RECORD OF TELEPHONE CONVERSATION
}

\author{
Date: 9-22-98 \\ Person Called: John Maziuk \\ Person Calling: Kevin Liekhus \\ Company: Church \& Dwight, Princeton, NJ \\ Phone No.: (609) 497-7282
}

\section{Subject: Sodium bicarbonate dry injection}

Mr. Maziuk stated that sodium bicarbonate $(\mathrm{NaBC})$ dry lime injection is typically operated at stoichiometric ratios equal or greater than 2 . It is important to maintain adequate cake thickness on the bags to achieve high efficiencies. A study was conducted to identify the effect of operating on acid gas removal efficiency. At gas flows on the order of 10,000 acfm, air-to-cloth ratios ranging from 4-4.6, $\mathrm{NaBC}$ stoichiometric ratio of approximately $2.5, \mathrm{HCl}$ removal efficiency ranged from $84-86 \%$. When fugitive air was eliminated from the gas stream, the total air flow was $8,250 \mathrm{acfm}$, air-to-cloth ratio decreased to 3.3-3.8, $\mathrm{HCl}$ removal efficiency increased to $94-98 \%$. When the sorbent fed was increased at the lower gas flow condition so that the gas flow (acfm)/sorbent feed rate $(\mathrm{lb} / \mathrm{hr}$ ) ratio was 50 (stoichiometric ratio between 3 and 4), removal efficiency of $99 \%$ was achieved. The $\mathrm{HCl}$ concentration was not specified, but for this last case, the $\mathrm{NaBC}$ feed rate is about $165 \mathrm{lb} / \mathrm{hr}$.

The pressure drop across the bags was maintained between 6-7 in. w.g. Moisture content of the offgas does not influence the scrubbing reaction. No vibrator was required on the $\mathrm{NaBC}$ bin to move solids. The baghouse solids collected were simply disposed of in a landfill. 


\section{RECORD OF TELEPHONE CONVERSATION}

Date: $9 / 14 / 98$

Person Called: Dan Leister

Person Calling: Kevin Liekhus

Company: Calgon Carbon

Phone No.: (412) 995-5500

Conversation: Mr. Leister stated that Calgon Carbon offered a sulfur-impregnated carbon (SIC) but it was not designed to operate at exceedingly high temperatures. Above $90 \mathrm{~F}$, sulfur (S) sublimation becomes an issue. Over a 5 -yr period, $50 \%$ of the $S$ sublimes at $140 \mathrm{~F}$. Sublimation losses can be reduced significantly at $90 \mathrm{~F}$. The carbon has the maximum capacity of approximately $20 \mathrm{lb} \mathrm{C} / 100 \mathrm{lb} \mathrm{C}$. The necessary gas contact with the $\mathrm{C}$ is on the order of $4 \mathrm{~s}$ based on the total actual gas flow rate. The SIC has a small mass transfer zone where the bulk of Hg removal occurs but there is a very long tail where $\mathrm{Hg}$ removal occurs much more slowly.

Of course, water condensation is undesirable for it inhibits gas transfer to the $\mathrm{C}$ surface but the performance of the SIC can be restored where the condensate is removed at higher temperatures. 
TRIP REPORT 10/1/98

KEVIN LIEKHUS

9/29/98 Birmingham, AL Children's Hospital (CH)

Met with Wes-he works at Bundy Environmental

Discussed $\mathrm{CH}$ operational conditions.

Inlet $[\mathrm{HCl}] \sim 500 \mathrm{ppmv}$, dry, $7 \% \mathrm{O}_{2}$

- Outlet concentration, $[\mathrm{HCl}]=9 \mathrm{ppmv}$, dry, $7 \% \mathrm{O}_{2},(\sim 98 \% \mathrm{HCl}$ removal $)$

Air-to-cloth ration $\sim 2.8: 1$

Waste feed rate $\sim 1000 \mathrm{lb} / \mathrm{hr}$

Hastelloy parts used in evaporative cooler to minimize corrosion

Operates $8 \mathrm{hr} /$ day. Baghouse isolated to minimize aifflow through it when not operating

Visited facility

Gravity-fed lime to screw feeder into venturi section of offgas line

Appears no effort to control, adjust lime feed for $\mathrm{HCl}$ removal

No active characterization of $\mathrm{Cl}$ in waste, $\mathrm{HCl}$ in offgas

Lime in storage bin, loaded by truck w/ blower

Baghouse maintenance appears to be negligible.

9/30/98 Apopka, FL BFI Medical Waste Incinerator

Facility burns bagged \& boxed waste $\sim 1900 \mathrm{lb} / \mathrm{hr}$

Incinerator doesn't appear to burn efficiently

Evaporative cooler has 3 atomizing nozzles (Hastelloy or Incoloy)

Corrosion of nozzles is minimized by covering nozzles with 316 SS shielding

Serves as a sacrificial shroud to inhibit erosion, corrosion

A drop out zone designed to increase distance for offgas to travel between evaporative cooler \& baghouse, and prevent water droplet carrier over to baghouse.

Facility runs $24 \mathrm{hrs} / \mathrm{day}-$ nearly one month

Shuts down for 2 days of maintenance

Again, air flow through baghouse is decreased to near zero during facility shutdown to minimize deliquescence of calcium chloride.

No glazing problems in baghouse

Fixed lime feed rate $\sim 50 \mathrm{lb} / \mathrm{hr}$

$\mathrm{HCl}$ removal efficiency based on 1991 data is 96\%

Design air-to-cloth ration is $3.9: 1$

Project manager (Jean Paradis) estimates baghouse solids collect at rate of $65 \mathrm{lb} / \mathrm{hr}(7 \%$ flyash)

I approximate their stoich metric ratio to be only 1.2

Apopka is under pressure/incentive to minimize waste from baghouse. This limited data suggest high $\mathrm{HCl}$ removal efficiency is achievable \& high moisture, longer contact times (lower air-to-cloth ratios), higher lime feed rates, result in better efficiencies.

Presently facility is having problems with material bridging in baghouse hopper. Manager believes it is related to incinerator operation.

Problems with bag filters service life related to over-pulsing of bags.

Facility was clean

Appears only a limited extra effort will be needed to handle issues related to lime/dust in a radioactive environment (extra dust containment). 


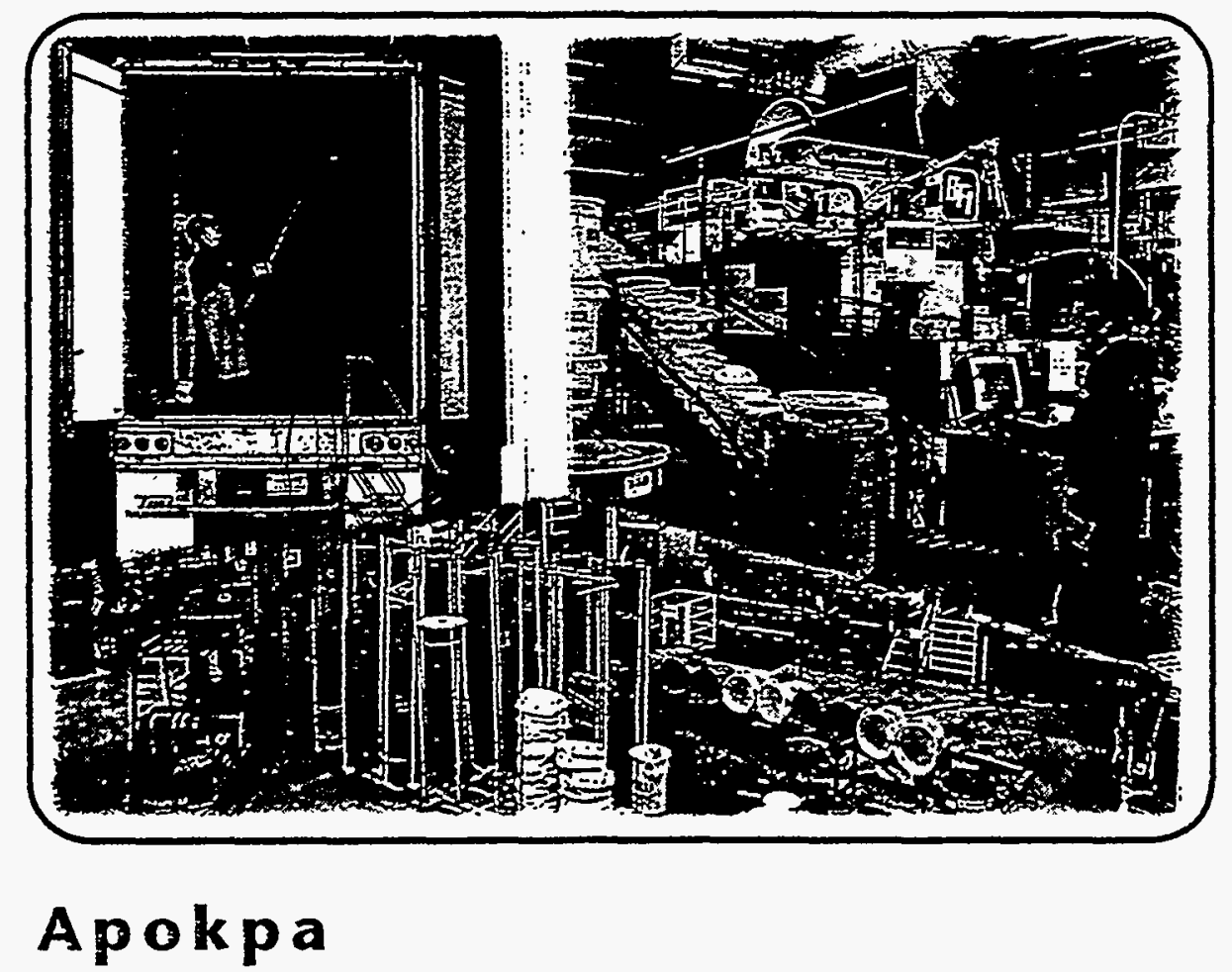

At a Glance

Technology Incinerator

Capacity $1,900 \mathrm{lbs} /$ hour

Processing Units One

Air Quality Control Dry Scrubber

Operating Hours $2+\times 7$
$\mathrm{BFI}$ is the nation's number one provider of Medical Waste collection and disposal services, with a network of over 25 processing facilities and 100 collection operations in the United States and Canada. BFI's Apopka Medical Waste Facility is located in central Florida and services the north/central Florida region. The facility, which has been in operation since 1994, includes a 16,788-sq. ft. steel building and processes approximately 15 million pounds of medical waste per year.

The Apopka facility utilizes a foy 2500 Dual chamber incinerator. This type of incinerator is known as a controlled-air incinerator that uses a system of two combustion chambers to promote complete burning of waste. In the primary chamber, air is distributed in controlled amounts so the quantity of air present is slightly less than that needed to complete combustion. The minimum temperature in this chamber ranges from $1600-1800^{\circ} \mathrm{F}$. This primary process generates a combustible, low-Btu fuel. Next, combustible gases enter the secondary chamber, where more air is added to cause a thorough mixing of combustible gases and air. The air/gas mixture in the secondary chamber burns at very high temperatures (ranging from $1800-2200^{\circ} \mathrm{F}$ ) to promote complete combustion. As gas leaves the final combustion chamber, it flows into the quench tower to reduce the temperature to $400^{\circ} \mathrm{F}$, and then into the final compartment, known as the baghouse. The purpose of the baghouse is to provide air quality control in an incinerator operation. Once introduced to the baghouse, untreated gases flow through woven fiberglass "socks" that act as filters. Dry lime is blown into the baghouse, it cakes onto the socks and behaves as a neutralizing agent to fiter gases even further. This baghouse system scrubs flue gases to levels well below local, state, and federal regulations. 
To insure continuous processing, containers are off-loaded from trailers onto a conveyor, the conveyor then advances the containers to a scale where they are weighed and recorded. The information recorded includes weight, date, time, customer information, and operator name. The larger roll off containers are emptied onto a process conveyor that moves the waste to the incinerator. After processing, the ash is taken to an FDEP approved and permitted sanitary landfill.

With more than 20 years experience in the Medical Waste business, BFI knows that accurate record keeping is vital to ensuring full compliance with medical waste regulations. To provide the highest degree of accuracy possible, the BFI Biotrack System uses portable and stationary computers, optical scanning devices, calibrated scales and bar-coded labels to track medical waste from the time it is collected until it arrives at our treatment facility for incineration. This automated approach eliminates the errors associated with handwritten entries and keeps the record of compliance updated on a continual basis.

All personnel at BFl's Apopka Medical Waste facility are trained and evaluated on personal protection equipment, proper handling of medical waste, appropriate operation of equipment, emergency response, spill control, and countermeasures.

BFl's goal at the Apopka Medical Waste facility is to provide the highest quality waste collection, transportation, processing, disposal and related services to both public and private customers. BFI will carry out this mission efficiently, safely and in an environmentally responsible manner with respect for the role of government in protecting the public interest.

\section{Facility Contact}

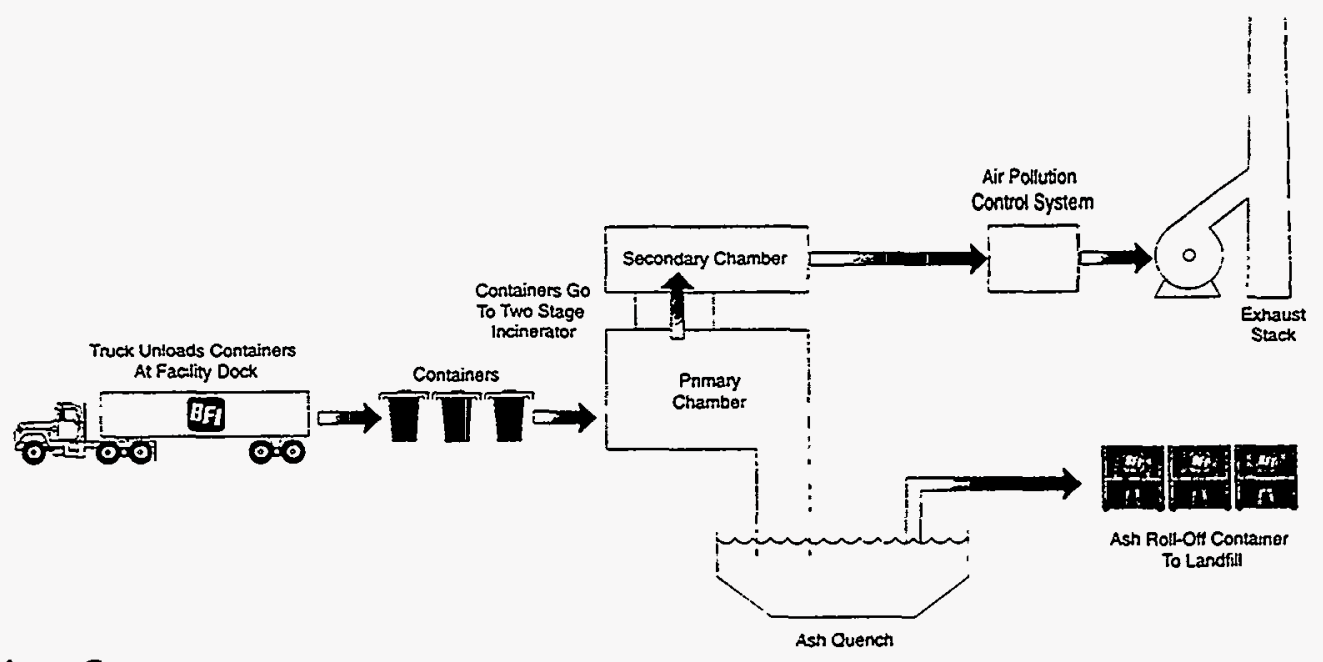

Facility Contact:

BFI Medical Waste Systems

Apopka Medical Waste Facility

254 West Keene Road

Apopka, Florida 32703

$800-825-4907$

(407)889-2800
Operating hours: $24 \times 7$

Holidays closed: New Year's Day, Memorial Day, Independence Day, Labor Day, Thanksgiving and Christmas Day 


\title{
APC System Case History
}

\author{
THE CHILDREN'S HOSPITAL \\ OF ALABAMA \\ Birmingham, Alabama
}

\section{Project Overview}

The Children's Hospital of Alabama installed a $1000 \mathrm{lb} / \mathrm{hr}$ fixed hearth Joy Energy Systems incinerator in 1991. In anticipation of future air pollution control regulations, space had been allowed and $a$ foundation poured for a wet scrubbing system to be added later.

By the summer of 1993 there were two items that caused the Hospital to reconsider their decision to use a wet scrubber. First, a ten (10) story parking garage and office building was being constructed on the adjacent lot, and a wet steam plume would be objectionable. Secondly, dry scrubbing was beginning to be the preferred technology by regulators and customers. The decision was then made to convert the design to a dry scrubber. Following intensive evaluation from six bidders, Bundy Environmental Technology was selected to provide the turnkey system.

The Hospital has no use for steam, and there was no heat recovery boiler to provide initial flue gas cooling. Instead, a dry bottom quench (or evaporative) cooler was used to reduce the incinerator flue gas temperature from a nominal $2000^{\circ} \mathrm{F}$ to $350^{\circ} \mathrm{F}$. Dry hydrated lime is injected just after the discharge from the evaporative cooler, and space has been allowed so that activated carbon injection for improved dioxin removal can be added in the future. A conventional BET pulse jet baghouse, equipped with GoreTex bags is used, with the normal accessories, i.e. top bag access, hopper heaters, level detector, vibrator, factory installed over the stiffener insulation and top insulation blankets.

The system has now been in operation for two (2) years and has provided an emission easily in compliance with the project requirements. While the customer had reservations initially about the possibility of plugging the baghouse or experiencing other problems due to water carry-over from the evaporative cooler, this has not occurred. The system has operated successfully and reliably, requiring only minimal, routine maintenance and repair.

\section{Design Data}

Inlet volume: $\quad 20,000 \mathrm{acfm}$

Inlet temp: $\quad 2100^{\circ} \mathrm{F}$

Reagents: Hydrated lime

Baghouse size: BET \#169-12
Schedule

System ordered: June 28, 1993

Construction: August 16, 1993

Operation: $\quad$ October 27, 1993

Tested:
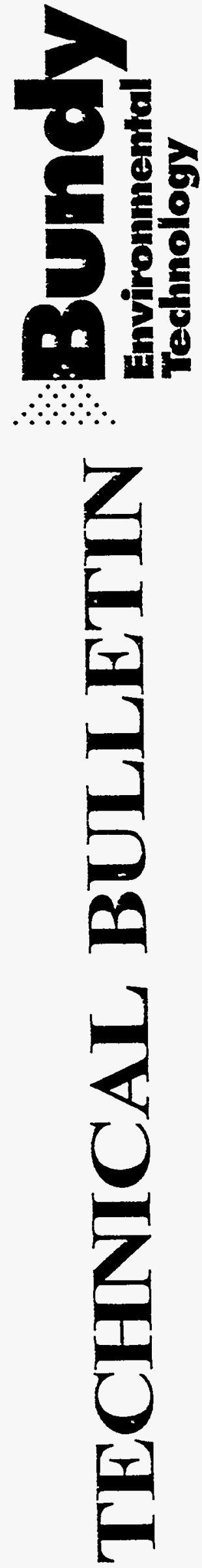

6950 - D Americana Pkwy Reynoldsburg, OH 43068 


\section{THE CHILDRE'S HOSPTTAL \\ OF ALABAMA}

\section{BET Scope of Supply}

- Incinerator Take-off: incinerator stack extension, counterweighted incinerator stack cap with hydraulic operator, breaching from the incinerator to the cooler, and refractory lining

- Evaporative Cooler: horizontal connection inlet plenum, inlet cone, cylinder, and discharge section with door, nozzles with air and water supply lances and accessories, and refractory and corrosion linings

- Reagent System: reagent storage silo with a live bin discharge, vent filter, and level indication; truck fill station, and variable flow volumetric feeder

- Reagent injection: gravity feed and proprietary venturi duct injector

- Pulse jet fabric filter (baghouse): GoreTex bags, hopper heaters, level detector, vibrator, top bag access, factory insulation, supports, access, and rotary airlock discharge valve

- Pumps and Compressed air system: water supply pump and back-up, water flow control valves, compressor with tank and accessories, desiccant dryer, compressed air flow control valves, all piped, wired, and mounted on a skid

- Flue gas valves and ductwork: baghouse inlet and outlet isolation valves, by-pass valve and interconnecting ductwork, volume (draft) control valve with air operated controller, system ductwork, and discharge stack

- Air moving equipment: I.D. fan, outlet damper, motor, drive and guard

- PLC control panel: with indication, controls and alarm, and disconnect and motor controls, and incinerator interface connections.

- Field construction: including foundations, erection, wiring, piping, insulation, and field painting

\section{Emission Test Results (Corrected to $7 \% \mathrm{O}_{2}$ )}

\begin{tabular}{llll} 
Pollutant & $\begin{array}{l}\text { Contract } \\
\text { Requirement }\end{array}$ & $\begin{array}{l}\text { Actual } \\
\text { Emission }\end{array}$ & $\begin{array}{l}\text { Actual } \\
\text { as a } \% \\
\text { of required }\end{array}$ \\
\hline & & & \\
Particulate & $0.03 \mathrm{gr} / \mathrm{dscf}$ & $0.0031 \mathrm{gr} / \mathrm{dscf}$ & $10.3 \%$ \\
$\mathrm{HCl}$ & $1 \mathrm{lb} / \mathrm{hr}$. & $\begin{array}{l}0.204 \mathrm{lb} / \mathrm{hr}=9 \mathrm{ppmv} \\
98.1 \% \text { removal efficiency }\end{array}$ & $20.4 \%$
\end{tabular}

ch'cha bq3'95

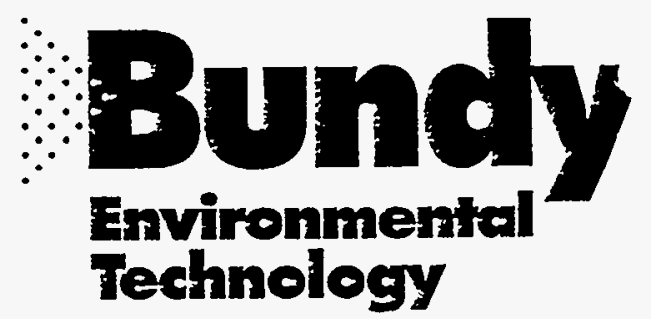




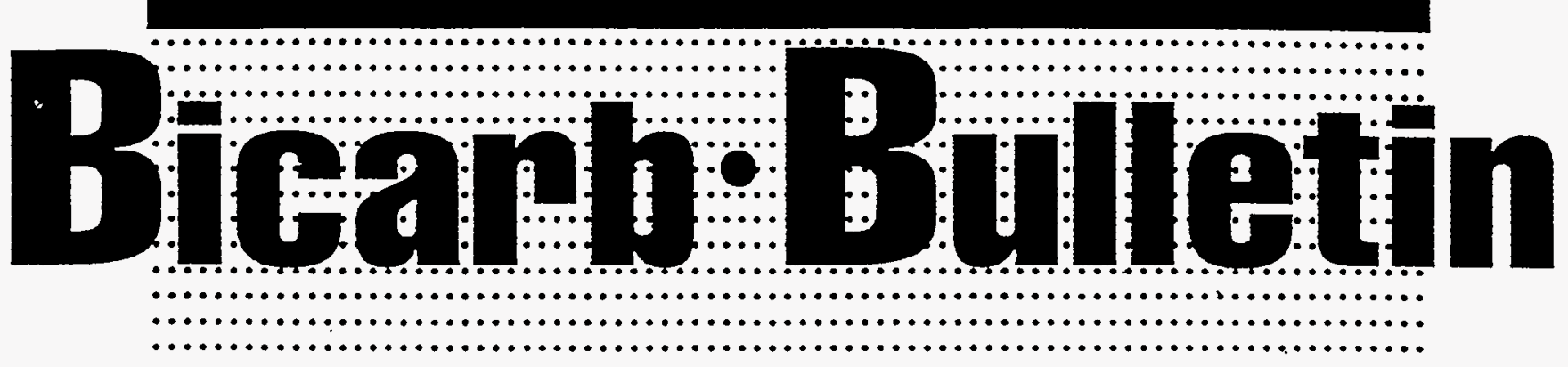

\section{DEACIDIFICATION OF MEDICAL WASTE INCINERATOR GASES WITH SODIUM BICARBONATE \\ $\mathrm{HCl}$ Removal with Sodium Bicarbonate Injection at \\ Swedish Medical Center, Seattle, WA}

\section{Case Background}

This report highlights a process employed by Swedish Medical Center (SMC) in Seattle, Washington, to optimally reduce hydrogen chloride $(\mathrm{HCl})$ emissions and other pollutants generated within the incineration process to comply with one of the world's most stringent air regulatory requirements.

SMC is a well-known 670-bed medical facility in a highly populared area. As part of its on-site medical waste incinerator operation, it relies on a dry sorbent injection-based air pollution control technology using ARM \& HAMMER ${ }^{\circledR}$ Sorbent Grade Sodium Bicarbonare. This technology assures SMC safely maintains air emissions from incinerated infectious and innocuous wastes generated from its daily operations.

The SMC is a registered air emission source within the City of Seattle and is subject to air regulations under the Puger Sound Air Pollution Control Agency (PSAPCA). As part of its etforts to maintain emissions within regulatory limits, SMC uses a sophisticated continuous emissions monitoring system (CEMS) which fearures an $\mathrm{HCl}$ Analyzer as a guide to control emissions by adjusting waste and sorbent feed. In 1991, following its use and evaluation of orher sorbent alternatives, SMC began using ARM \& HAMMER ${ }^{\circledR}$ Sorbent Grade Sodium Bicarbonate. Since doing so, the average $\mathrm{HCl}$ removal rate at SMC is 98.5 percent (See Table I below).

\section{TABLE I SWEDISH MEDICAL CENTER STACK TEST RESULTS (EPA METHOD 26)}

\begin{tabular}{|c|c|c|c|c|}
\hline \multirow[b]{2}{*}{ Stack Data } & \multicolumn{3}{|c|}{ RUN NUMBER } & \\
\hline & 1 & 2 & 3 & AVG. \\
\hline Volumetric Flow (ACFM) & 3446.0 & 3687.1 & 3772.7 & 3635.3 \\
\hline Volumetric Flow (DSCFM) & 1939.6 & 2070.1 & 2079.9 & 2029.9 \\
\hline Temperature $\left({ }^{\circ} \mathrm{F}\right)$ & 346.2 & 349.7 & 357.4 & 351.1 \\
\hline Moisture (Vol.\%) & 1219 & 11.97 & 12.47 & 12.21 \\
\hline \multicolumn{5}{|l|}{ Particulate gr/dsct @ 7\% O } \\
\hline PM & 0.008 & 0.013 & 0.005 & 0.009 \\
\hline \multicolumn{5}{|l|}{ Hydrogen Chloride @ $7 \% \mathrm{O}_{2}$} \\
\hline $\begin{array}{l}\text { Srubber Iniet (ppm) } \\
\text { Stack (ppm) } \\
\text { HCI Removal Efficiency }\end{array}$ & $\begin{array}{l}1671 \\
16.5 \\
99.0\end{array}$ & $\begin{array}{r}1472 \\
21.1 \\
98.6\end{array}$ & $\begin{array}{r}1526 \\
30.2 \\
98.0\end{array}$ & $\begin{array}{r}1556.3 \\
22.6 \\
98.5\end{array}$ \\
\hline
\end{tabular}




\section{System Description / Operation}

SMC employs a professional engineering staff to operate its medical waste incinerator (MWI) and hear recovery boiler system. The staft runs the system continuously five days per week and initiares a five-hour burn-down early Saturday moming to allow the incinerator to cool for the remainder of the weekend. The operational cycle is reestablished each Monday after performing a "preventative maintenance" clean-up of the incinerator, boiler tubes and an inspection of the air ports and appropriate instrumentation.

The facility incorporates an incinerator that is specified for an 800-pound-per-hour waste feed capacity with ripical operating feed rates averaging 400 pounds per hour. Medical waste is manually fed into the $1400^{\circ} \mathrm{F}$ primary chamber for gasification. The flue yases are then discharged into the afterburner (secondary chamber) and are iurther heated to $1650^{\circ} \mathrm{F}$ using natural gas as an auxiliary fuel source. (Reference SMC plant flow diagram).

The flue gases are then discharged into a waste hear boiler for heat recovery and cooling in preparation for dry sorbent injection and APCD filtration.

The cooled boiler flue gas enters the reaction chamber at approximately $400^{\circ} \mathrm{F}$ where sodium bicarbonare $\left(\mathrm{NaHCO}_{3}\right)$ is metered and pneumatically injected into the waste gas stream. The combined flue gas and reactive sodium bicarbonate then enter the fabric filter at $400^{\circ} \mathrm{F}$ where turther neutralization occurs and particulate matrer is collected. The total surface area for flue gas filtration and absorption across $16 j$ filter bags is approximately 2000 square feet.

Throughour the process, an SivlC operator continually monitors the $\mathrm{HCl}$ Analyzer. If any rise in $\mathrm{HCl}$ readings occur. the waste feed rate is reduced while the sorbent feed is increased to enhance acid gas removal.

\section{Swedish Hospital Incinerator System Process Flow Diagram}

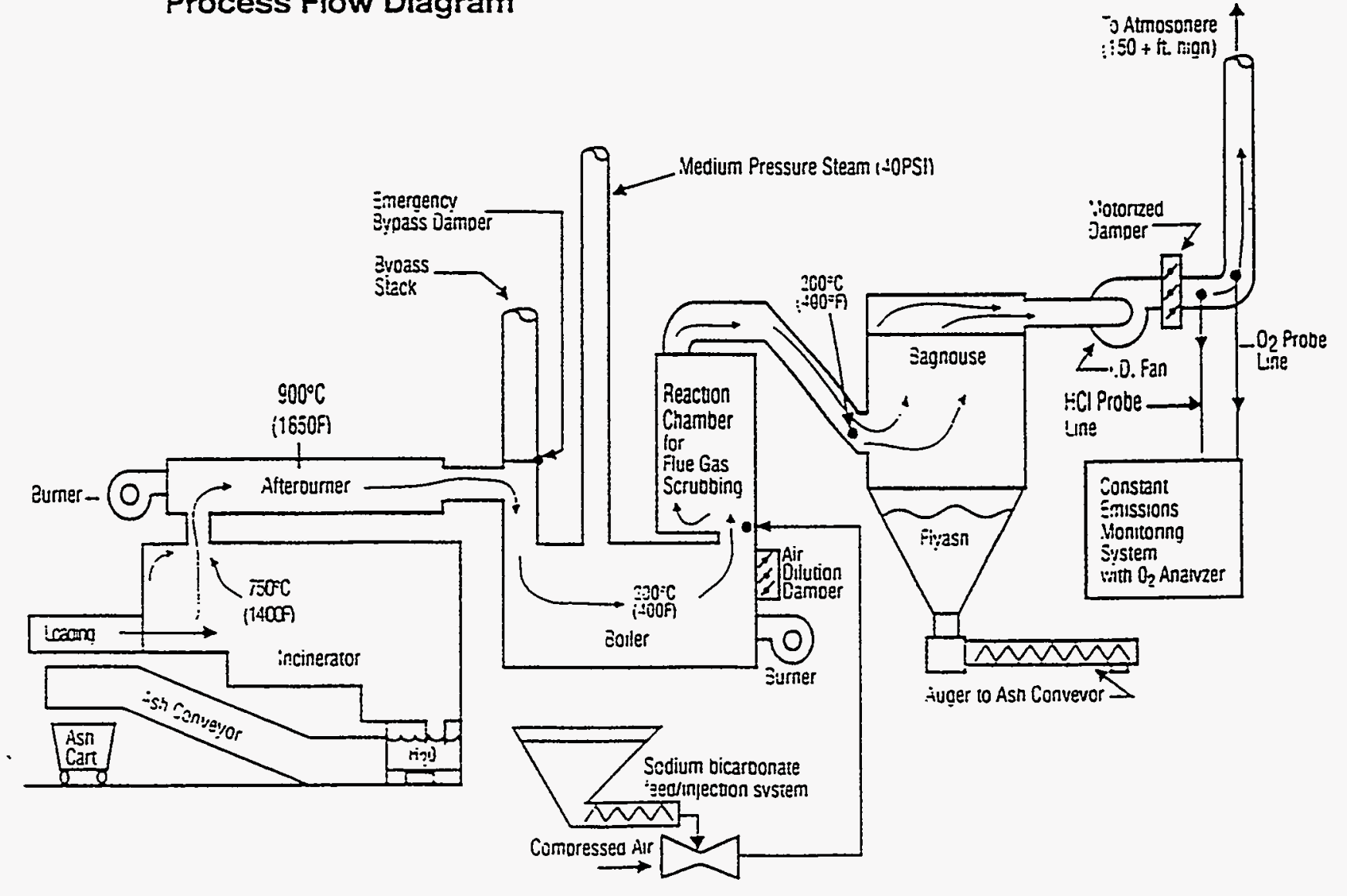




\section{System Description / Operation Continued...}

The sorbent feed rate is adjusted manually to achieve responsive control. The $\mathrm{HCl}$ Analyzer readings are logged continuously and printed out in 5 -minure intervals to maintain a $50 \mathrm{ppm}$ (corrected for $7 \% \mathrm{O}_{2}$ ) time averaged hourly output. In addition to preventing emission spikes and maintaining consistent control, this technique enables SMC to utilize only that amount of sorbent necessary to achieve required emissions levels.

\section{Sorbent Stoichiometry / Reaction Mechanism}

Upon injection into the hot flue gas stream, sodium bicarbonate undergoes rapid thermal decomposition. This resules in a highly porous, high surface area particle which has significant iffinity to neutralize acidic emissions such as $\mathrm{HCl}$. As particle porosity increases, acidic gases continually react with tresh reagent surtace to achieve maximum scrubbing. Acid-based neutralizarion of $\mathrm{HCl}$ is represented in the following stoichiometric reaction:

$\mathrm{NaHCO}_{3}+\mathrm{HCl} \longrightarrow \mathrm{NaCl}+\mathrm{CO}_{2}+\mathrm{H}_{2} \mathrm{O}$

Water vapor and carton dioxide produced by this reaction vent to the atmosphere through the the. The neutral salt reaction product (sodium chloride), along with the tly ash and unreacred surbent, collecr as filter cake on the fabric filters in the baghouse.

\section{Results}

Through injection of sodium bicarbonare, $\mathrm{HCl}$ emissions were reduced by an average of 98.5 percent based on Washington State certified stack test results (Reference Table I). As a result, SMC operates its facility well within the compliance requirements of one of the world's most stringent air regulatory bodies, while maintaining environmentally sound operation.

Compared to other sorbents, sodium bicarbonare results in high utilization efficiencies, improved baghouse performance due to reduced prressure drop, and improved operational and mechanical reliability due to its non-corrosive, non-erosive nature. SMC also reports its fabric filter bags last longer due to the sodium bicarbonare's high level performance of acid gas neutralization.

At the same time. the lower spent sortent volume reduces disposal costs and its negative environmental imp̣act.

SiMC's effective use of an $\mathrm{HCl}$ Analy:er is beneticial in monitoring and maintaining sare thue gas emissions levels by helping to control the waste teed rate, optimize sorbent urilizarion and prevent emission spikes.

Other norable advantages observed ky S.MC include the assurance of worker safert. easy-to-handle neutral salr by-products and improred particulate control device performance. While satery is alwars a prime necessiry, SivC's use of sodium bicarconate is especially beneticial beciuse the incinerator is housed in a location of limited space.

The data and experience ar SMC ralidates that by using ARM $\mathbb{E}$ HAMMMER ${ }^{\circledR}$ Surbent Grade Sodium Bicariconate, a dri scrubber APCD can operare efficiently and effecrively. 


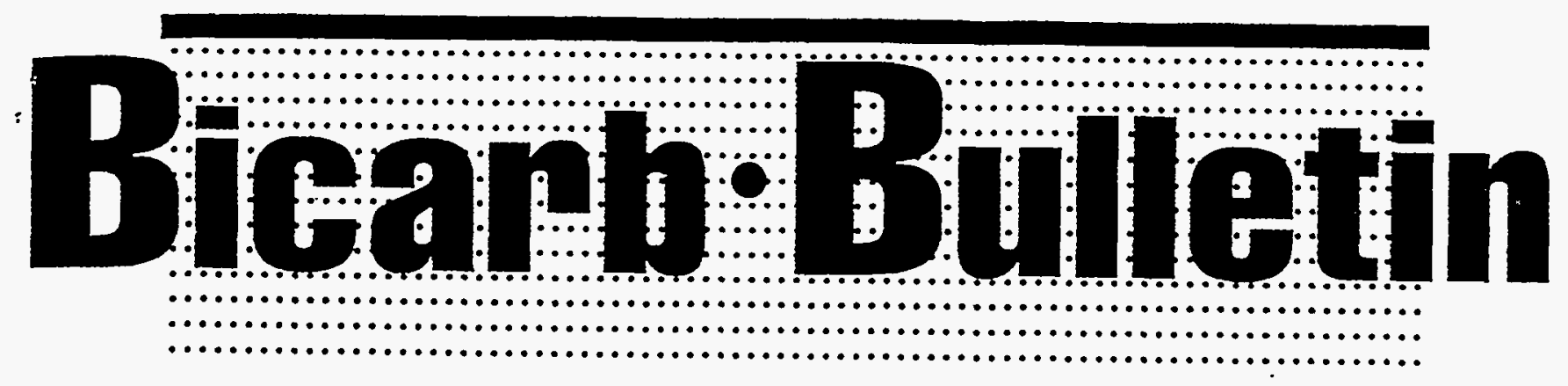

\section{DEACIDIFICATION OF MEDICAL WASTE INCINERATOR GASES WITH SODIUM BICARBONATE \\ $\mathrm{HCl}$ Removal with Sodium Bicarbonate Injection at \\ Colorado Incineration Services, Inc., Denver, CO}

The efficacy of sodium bicarbonate in reducing $\mathrm{SO}_{2}$ and other acid pollutants from stack gases is ivell-known.

This report highlights a process that optimally reduces hydrogen chloride $(\mathrm{HCl})$ emissions and other pollutants below that characteristically observed from medical waste incinerators (MWIs) with dry sorbent injection/fabric filter air pollution control device (APCD) technology. Using ARM \& HAivMER ${ }^{\circledR}$ Sorbent Grade Sodium Bicarbonate, $\mathrm{HCl}$ removal at the Colorado Incineration Services, Inc. (CISI) facility, near Denver, Colorado, was 99.3 percent.

\section{Case Background}

CISI was incorporated in 1989 to handle and dispose of infectious waste according to the guidelines ser by Colorado's New Source Pertormance Standard (NSPS) for MWI emissions. In June 1994. CISI ceased incineration operations. However, the dara remains both valid and valuable from which similar operations can benefit.

CISI provided medical waste incineration services to hospitals, doctors' offices and clinics throughout Colorado, Wyoming, Nebraska and New Mexico. Additional materials were handled from pharmaceurical manufacturers and laboratories, industrial clinics and dental offices. 


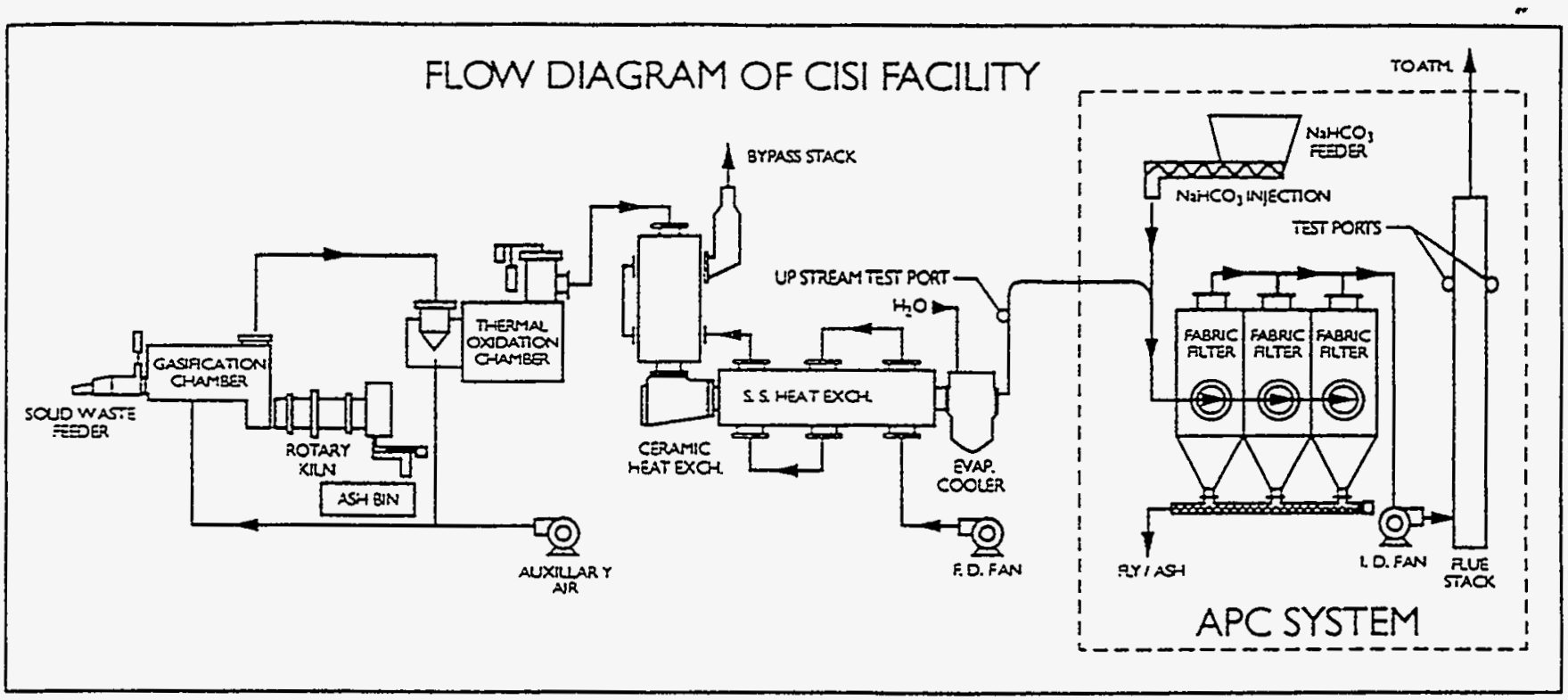

\section{System Description}

For its size (750-pounds-per-hour) CISI used a slightly differenc process than most cunventional WIWIs by operating in a continuous cycle rather ihan in barches. The tacility operated continuously, going to stand-by mode when the APCD was pulsed down and the fabric filter was precoated. After baghouse pulsing and precoating, yas flow was re-established to the APCD.

The facility used a dual chamber, starved-air incinerator (Reference CISI plant flow diagram). The solid waste feeder supplied waste to a stationary hearth gasifier (primary chamber) which uperated at $1600^{\circ} \mathrm{F}$ and used a 29-foor rotary ash kiln to gasify 95 percent of the solid waste.

Volatile organic compounds (VOCs) produced during this process were dissipared in a thermal uxidarion chamber. This secondary chamber uperated between $2150^{\circ} \mathrm{F}-2300^{\circ} \mathrm{F}$ with 100 percent excess air. Flue gases from this chamber intered five cross flow, air-to-air tubed hear exchangers (nvo ceramic and three stainless steel) and cooled to $375^{\circ} \mathrm{F}$. Pollutant-laden yases then entered the APCD for pollurion reduction.

There were five caregories of pollutants generated hy the medical waste. First was metals, which included arsenic, beryllium, cadmium, chromium, lead, mercury and nickel. The second type was chlorine isomers. The last three categuries were HCl. carbon monoxide and narticulate matter.

\section{APCD Operating Mode}

Pollutant-laden gases entered the APCD, which consisted of a sodium bicarbonate-injected fabric filter system that used 168 bays divided among three chambers, tor a total filtration surface area of 1385 square feet.

To reduce pollutant emissions below that considered acceprable by most regulators and APCD manufacturers. CISI applied and maintained a sudium bicarbonare $\left(\mathrm{NaHCO}_{3}\right)$ precoar injection onto the fabric tilter bags during the entire burn cricle.

\section{ARM \& HAMVIVR ${ }^{\circledR}$ Sorbent Grade Sodium Bicarbonate was metered and pneumatically injected into the waste gas stream at $350^{\circ} \mathrm{F}$ to $375^{\circ} \mathrm{F}$, and thermally decomposed for maximum scrubbing. The injection of dry sorbent at a rate of 38.5 pounds per hour fully neutralized the $\mathrm{HCl}$, based on a maximum waste output of 10 pounds per hour.}

The baghouse operated on an automatic differenrial pressure $(\Delta P)$ cleaning cycle which acrivated when pressure drop across each filtration chamber rose above eighe inches of $\mathrm{H}_{2} \mathrm{O}$. Operators could concrol the cleaning cycle by manually overriding the automatic sistem. This cleaning cicle began with a one-hour burn down, during which the waste feed was terminated, which lowered pollutant emissions across the APCD. 
All chambers were fully pulsed to remove fly ash and residue. A precoar layer of sodium bicarbonate was then injected into the baghouse to re-establish a filter cake of approximarely $1 / 64$ of an inch on the fabric filter surface (CISI's system requires a toral of 200 pounds $\mathrm{NaHCO}_{3}$ to achieve this precoar). Such precoating resulted in a pressure drop of less than 0.4 inches of $\mathrm{H}_{2} \mathrm{O}$ across the APCD.

The baghouse was then put on-line and sodium bicarbonate injection resumed to a 38.5 pounds per hour level as waste feed was re-established. The system typically operated for 12 hours of continuous service before a differential pressure-based cleaning cycle (described above) was required.

\section{Sorbent Stoichiometry / Reaction Mechanism}

Upon injection into the hot flue gas stream, sodium bicarbonate undergoes rapid thermal decomposition. This results in a highly porous, high surface area particle which has significant atfinity to neutralize acidic emissions such as $\mathrm{HCl}$. Acid-based neutralization of $\mathrm{HCl}$ is represented in the following stoichiometric reaction:

$$
\mathrm{NaHCO}_{3}+\mathrm{HCl} \longrightarrow \mathrm{NaCl}+\mathrm{CO}_{2}+\mathrm{H}_{2} \mathrm{O}
$$

Water vapor and carbon dioxide produced by this reaction vent to the atmosphere through the tlue. The neutral salt reaction product (sodium chloride), along with the tly ash and unreacred surbent, collect as filter cake on the fabric tilters in the baghouse.

\section{Results}

Through injection of sodium bicarbonate, $\mathrm{HCl}$ emissions were reduced by an average of 99.3 percent based on Colorado Stare certified stack test results (Reference Table I).

In addition, wher pollutants such as particulate, metals and dioxins were well within stare regulatury requirements (Reference Table II).

Compared to other sorbents, sodium bicarbonate results in high urilization efficiencies, improved haghouse pertormance due to reduced pressure drop, and improved operational and mechanical reliability due to its non-comosive, non-erosive narure. At the same time, the reduced spent sorbent volume decreased disposal costs and minimized the negative environmental impact.

Other notable advantages observed by CISI included the assurance of worker safery, easy-to-handle neutral salt by-products and improved particulate control device performance.

By using ARM \& HAiVIMER ${ }^{\circledR}$ Sorbent Grade Sodium Bicarbonare, data collected demonstrate that a dry scrubber APCD can operare extremely efficiently with minimal system modifications.

TABLE I

GISI STACK TEST RESULTS (EPA METHOD 26)

\begin{tabular}{|c|c|c|c|c|c|}
\hline \multirow[b]{2}{*}{ Stack Data } & \multicolumn{4}{|c|}{ RUN NUMBER } & \multirow[b]{2}{*}{ AVG. } \\
\hline & $T$ & 2 & 3 & 4 & \\
\hline Volumetnc flow (ACFM) & 7569 & 8062 & 6716 & 7936 & 7571 \\
\hline Volumetric flow (DSCFM) & 3988 & 4107 & 3458 & 4141 & 3924 \\
\hline Temperature $\left({ }^{\circ} \mathrm{F}\right)$ & 345 & 354 & 354 & 356 & 352 \\
\hline Moisture (Vol.\%) & 4.3 & 6.4 & 5.5 & 4.0 & 5.1 \\
\hline \multicolumn{6}{|c|}{ Hydragen Chloride $\left(\Theta 7 \% \mathrm{O}_{2}\right)$} \\
\hline Srubber Inlet (ppm) & 807 & 1022 & 1152 & 905 & 1009 \\
\hline -Stacx (ppm) & 6.6 & 7.7 & 8.3 & 5.4 & 7.1 \\
\hline HCI Removal Efficiency & 99.2 & 99.2 & 99.3 & 99.4 & 99.3 \\
\hline
\end{tabular}

- Department of Enrironmental Conservation

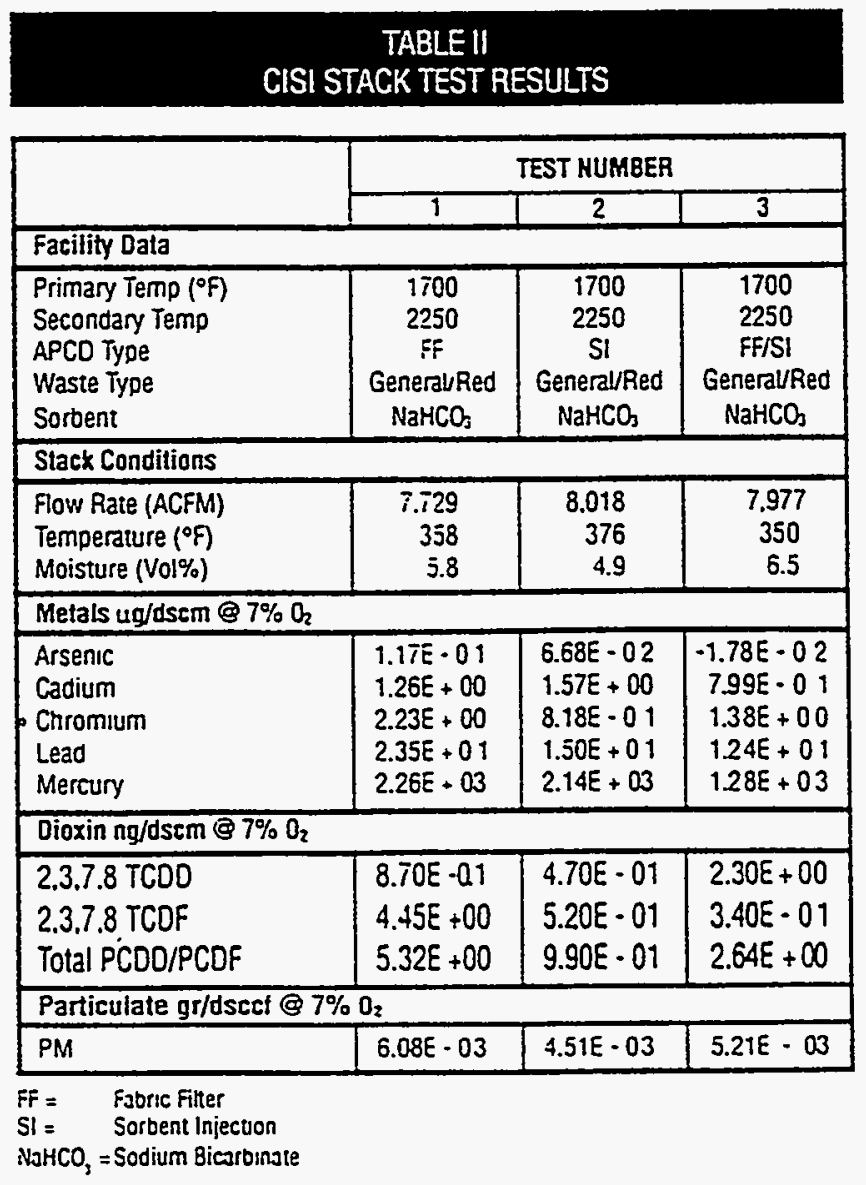




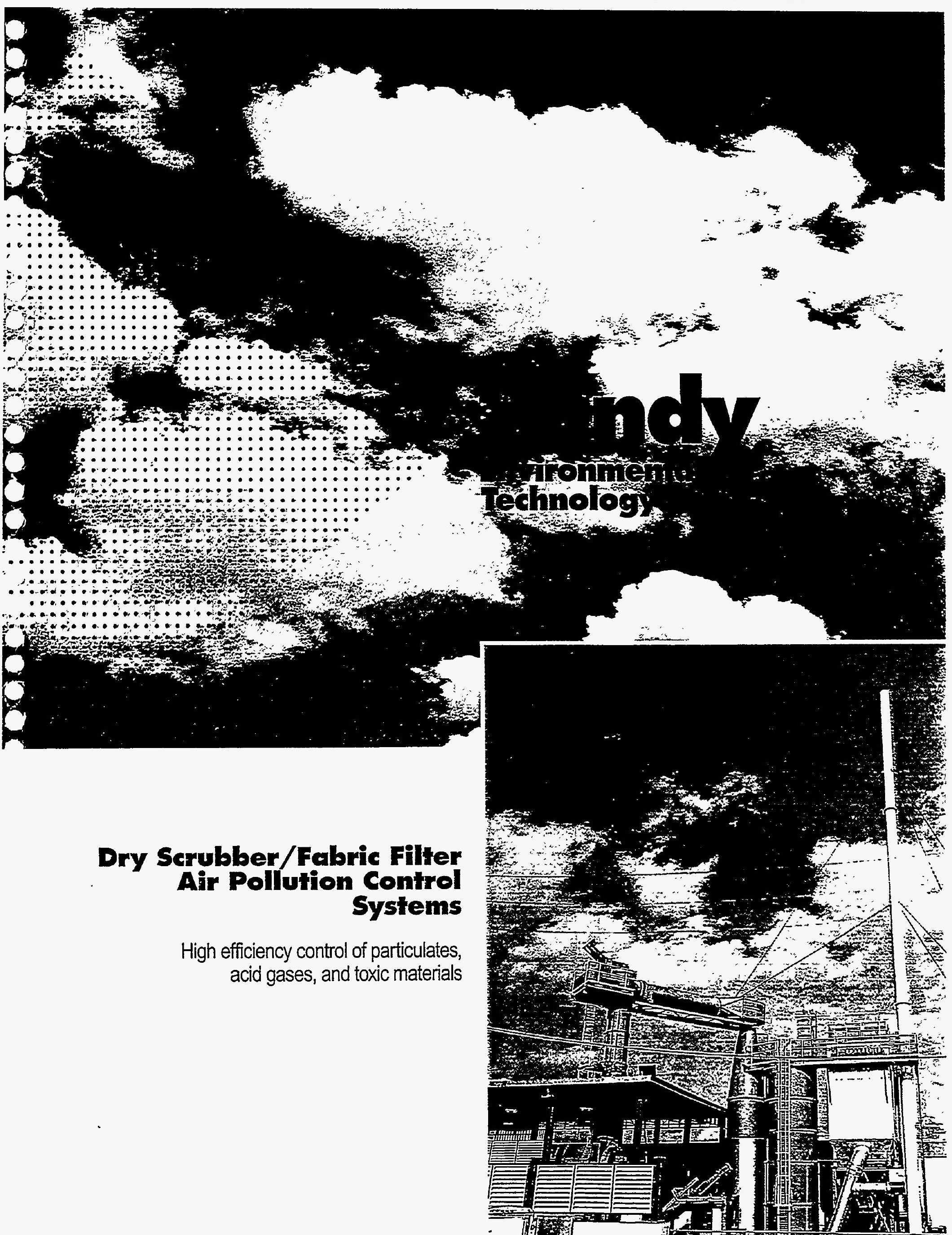




\section{Tighter standards, tougher compliance}

New demands have been placed on many of the smaller combustion sources for highly efficient air pollution control. Systems using only controlled combustion or low-efficiency wet scrubbers may be out of compliance. Facility owners need help, but are often presented with inadequate solutions: costly down-scaled systems that were designed for large municipal incinerators, poorly designed upgrades of dust collector baghouses, and inefficient add-ons.

At Bundy Environmental Technology, our sole business is to solve these problems. We design. fabricate and install cost competitive fabric filterbased pollution control systems that.are sized and configured to your exact requirements. You get reliable. high efficiency removal of particulates, acid gases, toxics, and heavy metals without costly overdesign or constant maintenance.

BET draws on 25 years of engineering experience and scores of successful installations to provide a compliant emission control system that is fully compatible with your incinerator and your operations.

\section{Bat offers a full line of Dry Serubber/Fabric Filfer Ststem allernalives.}

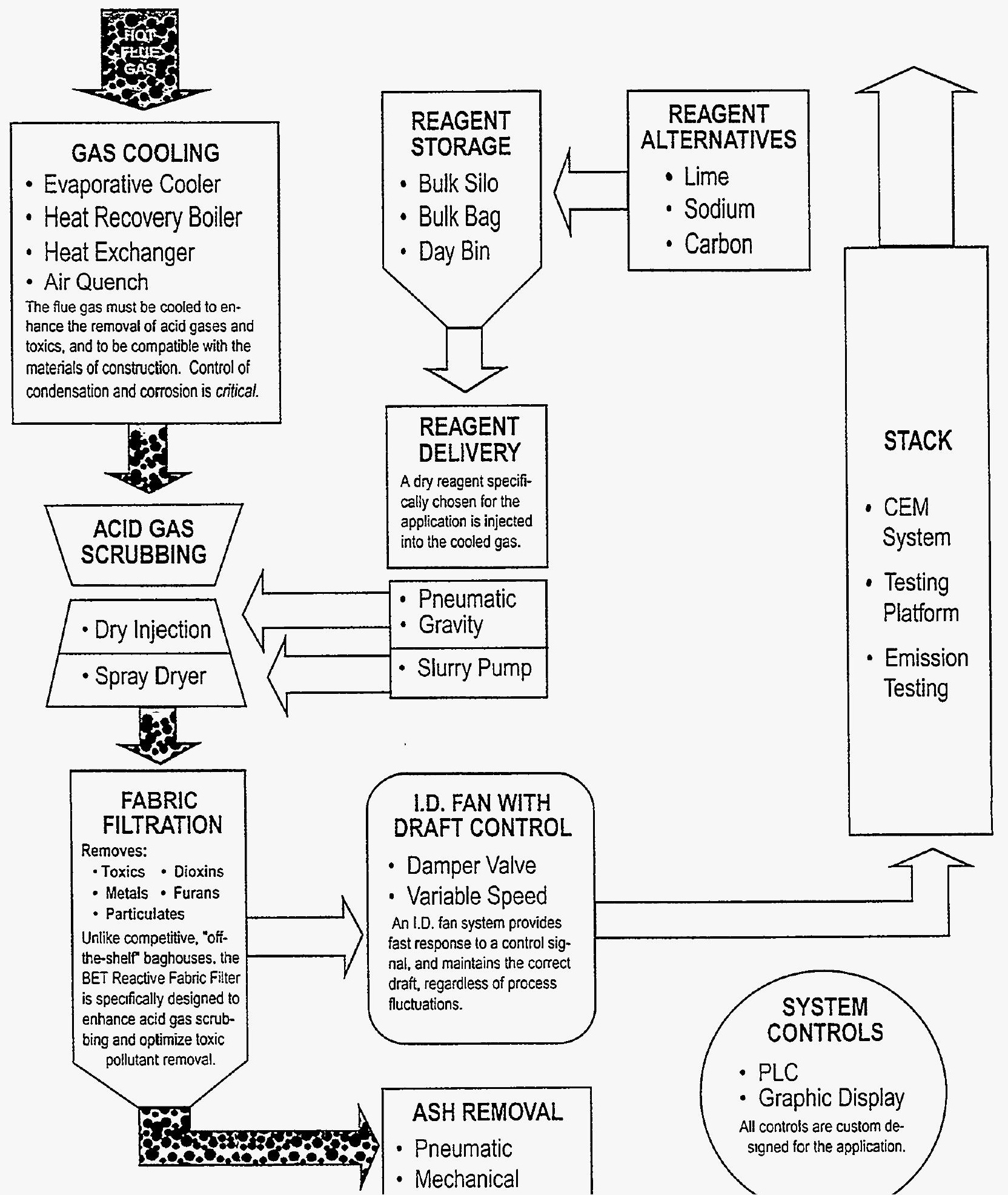




\section{Big ststem relfablith Small ststem costo}

Small air pollution control systems must be every bit as durable and reliable as those used with giant municipal incinerators and utility boilers. While some companies try unsuccessfully to scale down their large systems to smaller applications, and others apply cheap underdesigned "dust collectors", BET provides the features and design that match perfectly with your performance requirements and your capital and operating budgets. You benefit from numerous quality enhancements, some of which cannot be found on even the most expensive air pollution control systems:

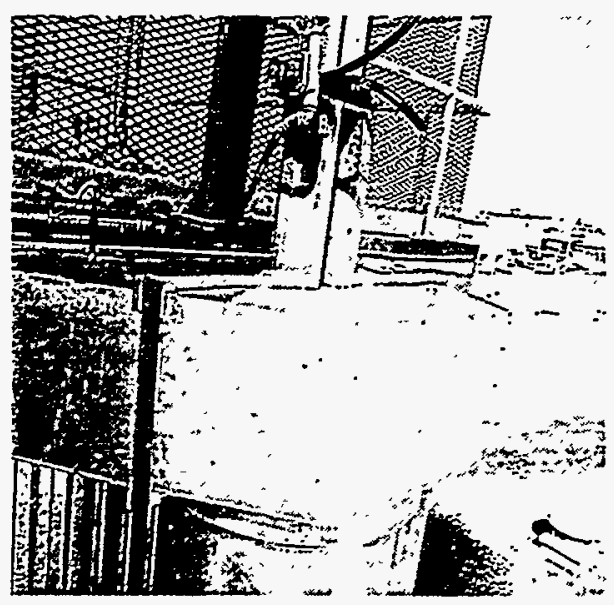

The baghouse is completely sealed from ambient air infiltration and condensation during off-line periods with an outlet poppet valve and inlet damper.
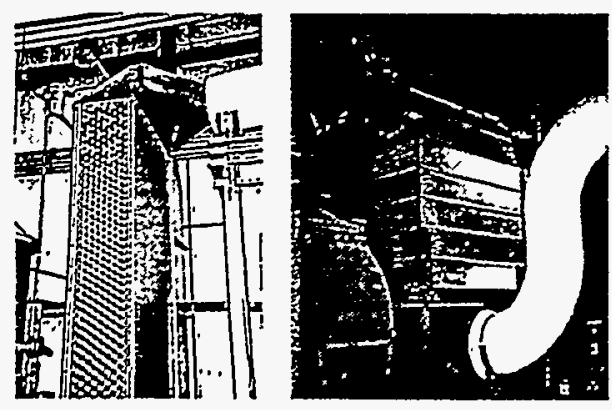

Heat exchanger shell and tubes are fully Teflon lined. They do not depend on surface preparation and material bonding as do epoxy-coated designs.

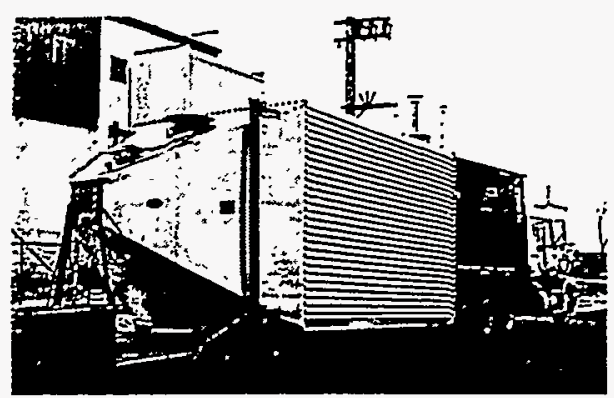

Baghouse insulation is applied over the stiffener and protected by box ribbed siding. All insulation is installed in the shop under controlled conditions for superior quality. Factory assembled baghouse ships as a single unit.

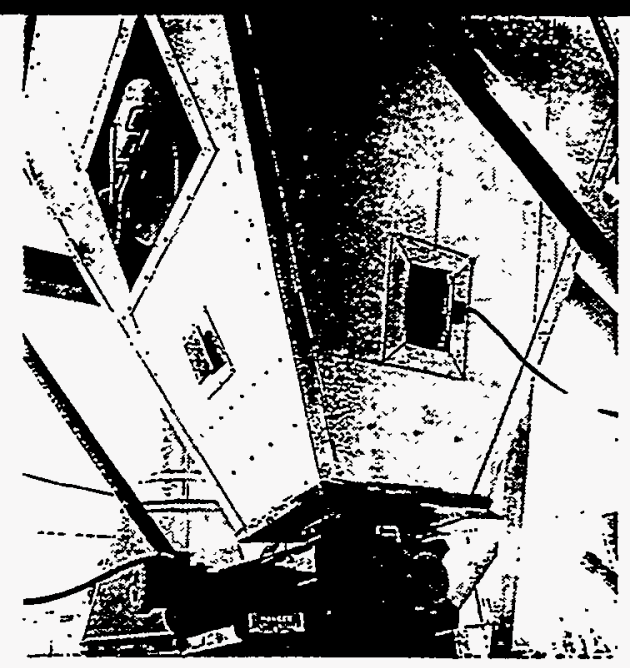

Baghouse hopper has steep walls and a number of accessories 10 assure smooth material discharge.

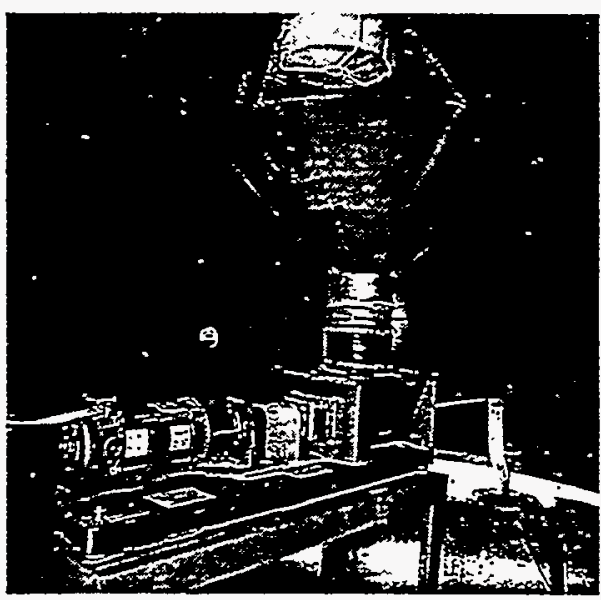

Continuous reagent flow is assured by a live bin bottom. A volumetric feeder allows adjustment of the feed rate.

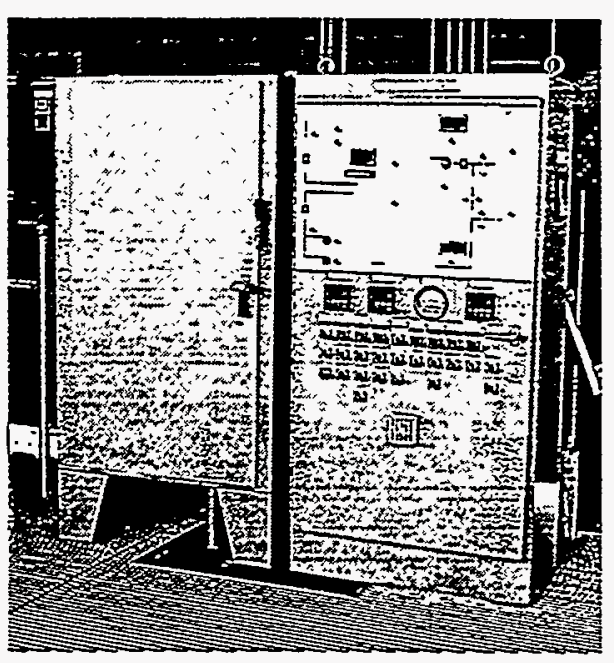

Controls are typically PLC based with individual component control loops, user-friendly graphic display of the total operation, and suitable indicating lights, gauges, and alarms.

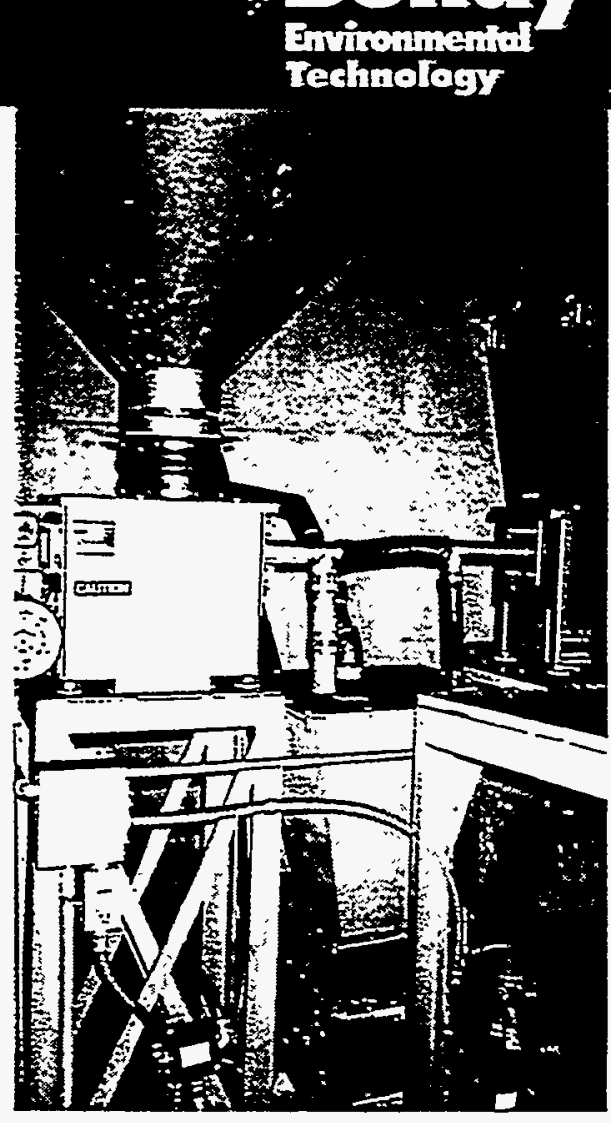

Multiple reagents are controlled independently, but continuously blended for control of all pollutants.

\section{Bundy clients include:}

- Automotive and steel plants

- Universities

- Hospitals

- Waste disposal companies

- Pharmaceutical firms

- Chemical plants

- Food and grain processors

- Lumber and paper mills

- Govemment and military facilities

- Lime and cement plants

- Soil remediation projects

\section{Experience with a variety of combustion sources:}

- Incinerators:

- Medical

- Industrial

- Hazardous

- Boilers:

- Spreader stoker, fluid bed, pulverized fuel, etc.

- Coal, wood, RDF, ag waste, etc.

- Furnaces

- Rotary kilns 


\section{Real world solutions with proven fedinology}

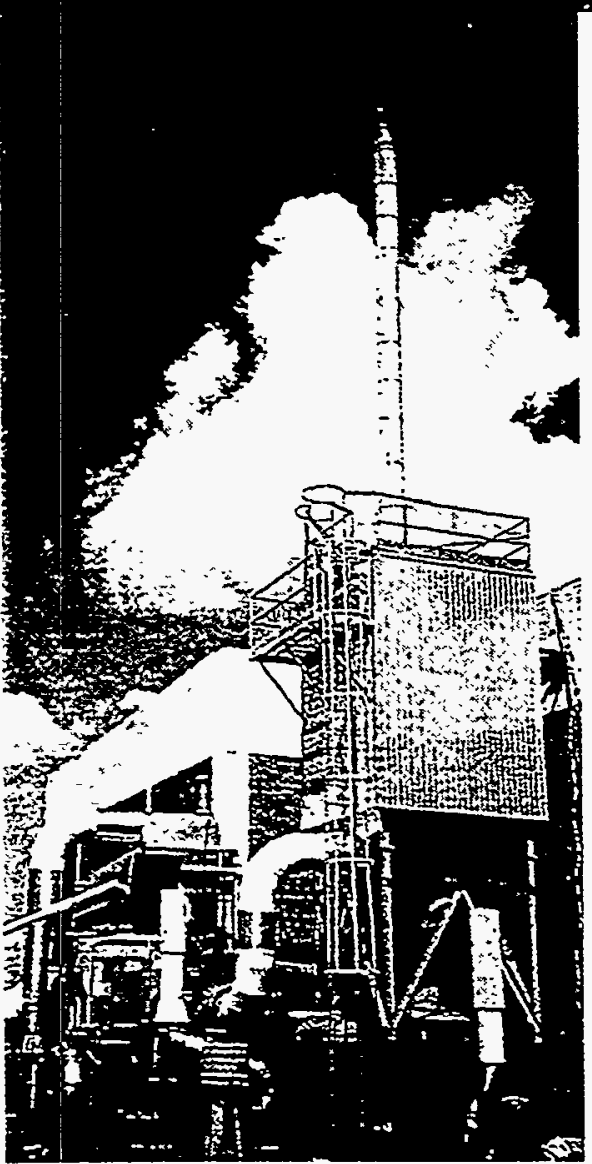

This New York state hospital operates a rotary kiln incinerator. BET furnished a tumkey system retrofit that successiully passed the most stringent emissions standards for particulates, acid gases, heavy metals, dioxins and other toxics.

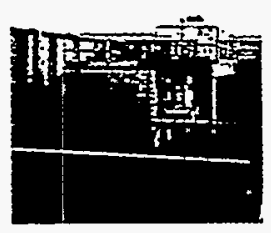

A commercial waste incinerator operates a BET heat exchanger, dry lime injection system, and two-module baghouse to comply with Michigan air pollution control standards.

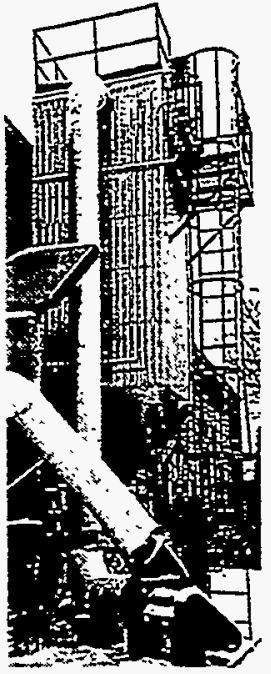

A Rhode Island hospital retroiftted a BET dry scrubber system to its existing $400 \mathrm{lb} / \mathrm{hr}$ incinerator and heat recovery boiler.

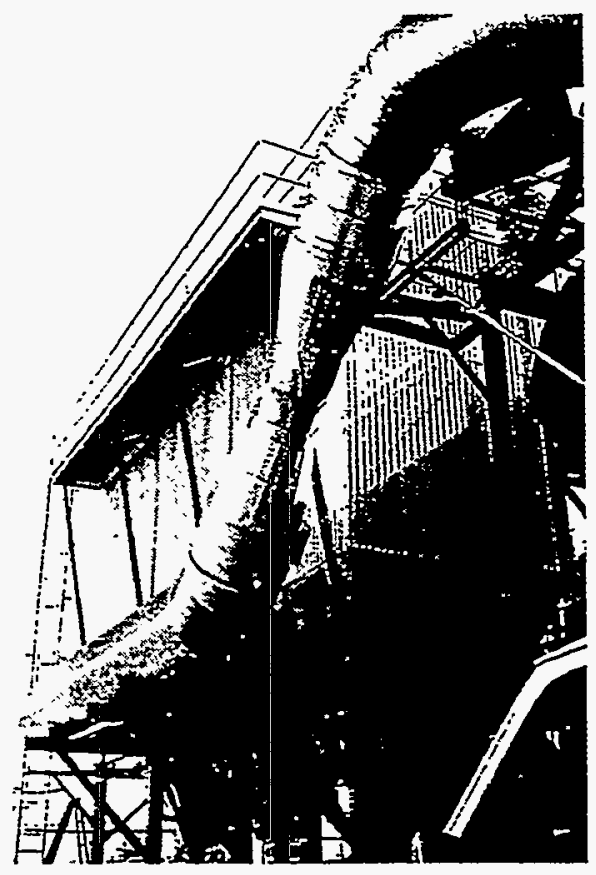

Multiple incinerators are manifolded together and ducted to this three-module dry scrubber/baghouse system in central Florida.

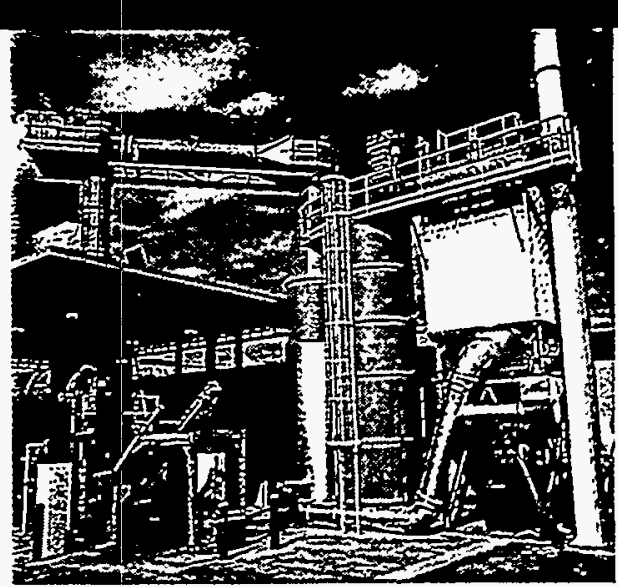

BET retrofitted a total turnkey air pollution control system ior this Alabama hospital: extension and cap for existing incinerator, breaching, evaporative cooler, dry scrubber, baghouse, draft conirol, fan, stack, controls, and all interfaces with the incinerator. (Also shown on cover.)

\section{Customized solutions to your problem}

Bundy Environmental solves air pollution contro problems one client at a time. You decide how much of our full project capability you need. We can design, manufacture, install, and test a complete turnkey system, or design and manufacture the equipment only. Should you wish to provide part of the system, we can furnish the remainder, taking care to insure total system integrity and compatibility.

BET does not deal with "off-the-shelf" solutions. We have no restrictive technological commitments or entangling license agreements. We are free to provide the exact pollution control techniques and equipment that will solve your problem most economically.

\section{Call for help.}

For honest answers from knowledgeable people whose only business is to solve air pollution problems, call:

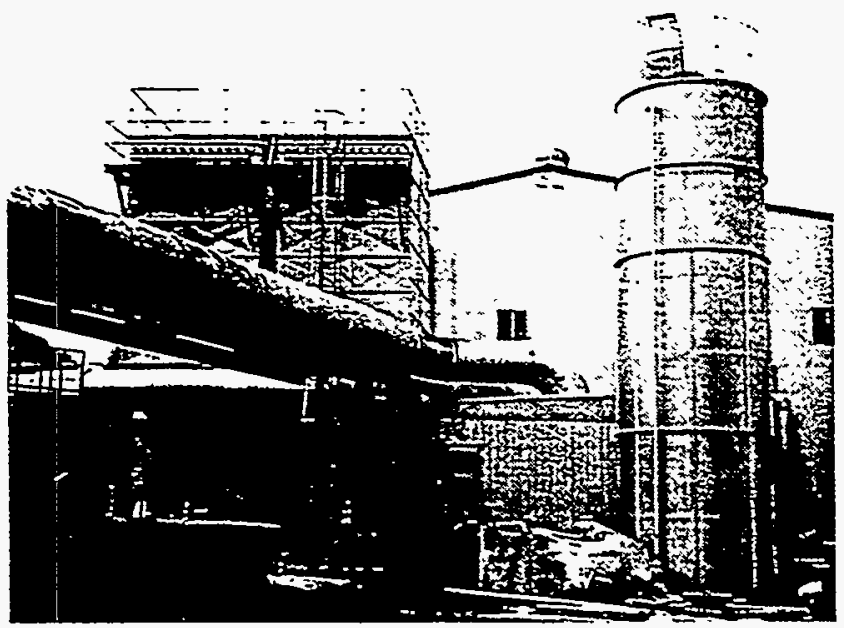

BET delivered all of the critical components for this Korean waste incineration facility, and provided the necessary drawings and specifications to enable the customer to locally source the steel fabrication.

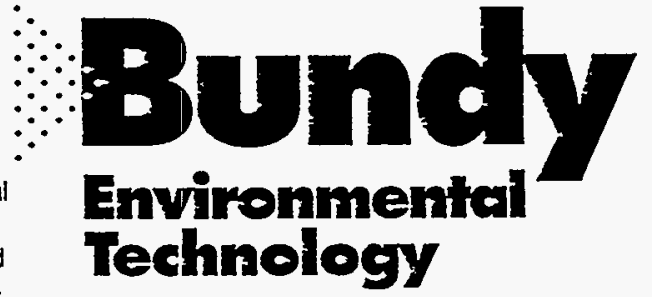

Bundy Environmental Technology, Inc.

6950-D Americana Pkwy.

Reynoldsburg, $\mathrm{OH}$ 43068-4127

(614) 861-2500 - Fax: (614) 861-2290 


\title{
APC System Case History
}

\author{
UNIVERSITY OF ROCHESTER \\ STRONG MEMORIAL HOSPITAL \\ Rochester, New York
}

\section{Project Overview}

Strong Memorial Hospital, part of the University of Rochester Medical Center, retrofitted a dry scrubber system by Bundy Environmental Technology to their $1500 \mathrm{lb} / \mathrm{hr}$ rotary incinerator to comply with the New York state regulations, 219-3. This system met all NYDEC emission limitations, including particulate, $\mathrm{HCl}$, dioxins and furans, mercury, and twelve (12) other individually named heavy metal and toxic pollutants, and after more than two (2) years of operation had not experienced a single bag failure.

The hospital had contracted with Cross/Tessitore and Associates (now part of Harding Lawson) to develop and implement plans to put them in compliance. C/TA evaluated alternative technologies and determined that dry scrubbing would be best to meet the current as well as anticipated future regulations. They solicited competitive bids and selected the BET system from the nine proposals received.

There were specific site conditions that made this a unique and challenging project:
- Space limitations and restrictions by the hospital dictated that the majority of the system be located on the roof of a seven story wing. The reagent storage and supply equipment would be on the ground, approximately $150^{\prime}$ away.

- This was one of the first medical waste incinerators to apply for permitting under the NYDEC Regulation 219-3, and it was assigned to the state, rather than the regional office, which caused considerable delays.

- Incinerator and boiler operations presented flue gas temperature fluctuations at the inlet to the system ranging from $450^{\circ} \mathrm{F}$ to $700^{\circ} \mathrm{F}$.

- Although regulation 219-3 only specifically names particulate, $\mathrm{HCl}$, opacity, and $\mathrm{CO}$ as pollutants to be controlled, during the permitting process, limits were set for thirteen (13) additional individual pollutants (see chart on pg. 4).

\section{Design Data}

Inlet volume: $\quad 10,000 \mathrm{acfm}$ Inlet temp: $\quad 450-700 \mathrm{~F}$

Reagents: Hydrated lime \&Activated carbon

Baghouse size: BET \#144-12
Schedule

System ordered: April, 1991

Construction: February, 1993*

Operation: June, 1993

Tested: . September, 1993

* Delay due to NY permit schedule
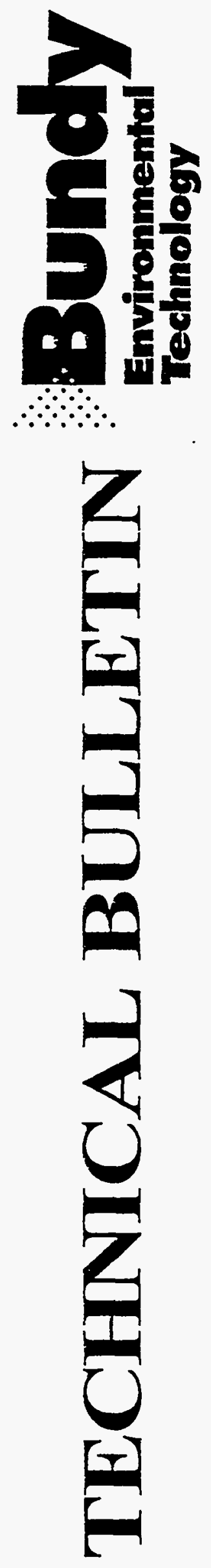

6950 - D Americana Pkwy Reynoldsburg. OH 43068

(614) $861-2500$ 


\section{UNIVERSITY OF ROCHESTER STRONG MEMORIAL HOSPITAL}

\section{BET Scope of Supply}

- Heat Exchanger: two stage, Teflon lined, sonic soot blower, modulating cooling control

- Reagent System: lime silo, carbon bin, independent variable speed volumetric feeders, ventilation systems, skirt and access

- Reagent Injection: pneumatic conveyor, proprietary venturi duct injector

- Pulse Jet Fabric Filter (Baghouse): P-84 bags, hopper heaters, level detector, vibrator, top bag access, factory insulation, supports, and access
- Ash Removal System: Double dump valve, pneumatic conveyor with heat tracing, receiver

- I.D. Fan with motor and variable speed drive

- Valves and Duct: Draft control valve, bypass valve, baghouse isolation valves, duct from the incinerator/boiler, discharge stack

- CEM System for $\mathrm{CO}, \mathrm{O}_{2}$, and opacity

- Construction: Mechanical erection, field wiring, piping, and insulation

\section{Emission Test results (Corrected to $7 \% \mathrm{O}_{2}$ )}

\begin{tabular}{|c|c|c|c|c|c|}
\hline POLLUTANT & $\begin{array}{l}\text { PERMT } \\
\mathrm{lb} / \mathrm{hr}\end{array}$ & $\begin{array}{l}\text { ACTUAI } \\
\mathrm{lb} / \mathrm{hr}\end{array}$ & $\begin{array}{l}\text { Actual } \\
\text { as } \% \text { of } \\
\text { Allowed }\end{array}$ & $\begin{array}{l}\text { lb/ton } \\
\text { of waste } \\
\text { burned }\end{array}$ & $\begin{array}{l}\text { Conc. } \\
\mathrm{mg} / \mathrm{nM}^{3}\end{array}$ \\
\hline Arsenic & $3.47 \mathrm{E}-06$ & $<1.94 \mathrm{E}-06$ & $55.9 \%$ & $<3.2 \mathrm{E}-06$ & $<2.5 \mathrm{E}-04$ \\
\hline Cadmium & $4.22 E-04$ & $<0.09 \mathrm{E}-04$ & $2.1 \%$ & $<1.5 \mathrm{E}-05$ & $<0.0012$ \\
\hline Chromium, tot & $1.46 \mathrm{E}-03$ & $<0.03 E-03$ & $2.1 \%$ & $<5.0 \mathrm{E}-05$ & $<3.9 \mathrm{E}-03$ \\
\hline Chromium +6 & $1.46 \mathrm{E}-05$ & $0.52 E-05$ & $35.6 \%$ & 8.7E-06 & $6.8 \mathrm{E}-04$ \\
\hline Lead & $4.35 E-02$ & $<0.003 E-02$ & $<0.1 \%$ & $<5.0 \mathrm{E}-05$ & $<3.9 \mathrm{E}-03$ \\
\hline Manganese & $8.55 E-04$ & $<0.04 \mathrm{E}-04$ & $0.5 \%$ & $<6.7 \mathrm{E}-06$ & $<5.2 \mathrm{E}-04$ \\
\hline Nickel & $3.75 E-04$ & $0.05 E-04$ & $1.3 \%$ & $8.4 \mathrm{E}-06$ & $6.5 E-04$ \\
\hline Mercury & $2.06 E-02$ & $<0.007 \mathrm{E}-02$ & $0.3 \%$ & $<1.2 \mathrm{E}-04$ & $<0.009$ \\
\hline Zinc & $1.43 \mathrm{E}-03$ & $0.30 \mathrm{E}-03$ & $21.0 \%$ & 5.0E-04 & 0.039 \\
\hline Benzene & $1.03 E-01$ & $<0.07 \mathrm{E}-01$ & $6.8 \%$ & $<0.012$ & $<0.9$ \\
\hline Formaldehyde & $1.54 \mathrm{E}-02$ & $0.14 E-02$ & $9.1 \%$ & 2.3E-03 & 0.182 \\
\hline $\mathrm{PAH}$ & $3.45 \mathrm{E}-04$ & $0.29 \mathrm{E}-04$ & $8.4 \%$ & 4.8E-05 & 0.0038 \\
\hline Dioxin/Furan(TEQ) & $5.13 E-10$ & $<2.66 \mathrm{E}-10$ & $52.9 \%$ & $<4.4 \mathrm{E}-10$ & $\begin{array}{l}<0.035 \\
\mathrm{ng} / \mathrm{nM}^{3}\end{array}$ \\
\hline $\mathrm{HCl}$ & 1.75 & 0.04 & $2.3 \%$ & $\begin{array}{l}99.82 \% \\
\text { Removal }\end{array}$ & $\begin{array}{r}2.64 \\
\text { ppmv }\end{array}$ \\
\hline $\begin{array}{l}\text { Particulate } \\
\text { gr/dscf }\end{array}$ & 0.03 & 0.0009 & $3.0 \%$ & & \\
\hline
\end{tabular}

ch'uor bq3'9s

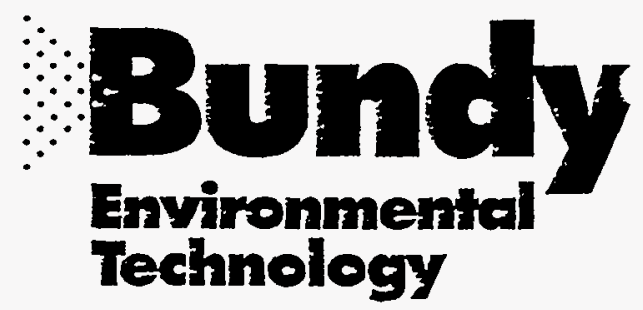

6950 - D Americana Pkwy

Reynoldsburg, $\mathrm{OH} 43068$

Phone (614) 861-2500

FAX (614) 861-2290 
Presented at the Air \& Waste Management Association's $90^{\text {th }}$ Annual Meeting \& Exhibition, June 8-13, 1997, Toronto, Ontario, Canada

97-FA166.03

\title{
Maximizing the Operating Efficiency of a Dry Scrubber on Medical Waste Incineration
}

\author{
By: \\ Richard P. Bundy \\ Bundy Environmental Technology Inc \\ 6950-D Americana Pkwy \\ Reynoldsburg, $\mathrm{OH} 43068$ \\ Phone (614) $861-2500$ \\ Fax (614) $861-2290$
}

\section{Introduction}

The Environmental Protection Agency has proposed standards for the emissions from medical waste incinerators as described in Table $1^{(1)}$. These standards are based on the best performing air pollution control systems which include both dry scrubbing systems and wet scrubbing systems.

Although there are dozens of dry scrubbing systems on medical waste incinerators, only five primary sites were included in the data provided by EPA as back up for these standards ${ }^{(2)}$. Since EPA established the standards based on the best performing systems, one would expect that these all would be relatively good installations, which in fact they are. The range of the emissions from these sites is shown in Table 2. There is a wide range of results. However, with only a few exceptions (especially true for $\mathrm{HCl}$ ) all perform within EPA's proposed standards. Other systems that were not included in the study have shown a greater range of emission levels particularly in terms of $\mathrm{HCl}$ scrubbing where efficiencies as low as 50 to $70 \%$ removal have been reported even when the systems were presumed to be in proper operation in accordance with the manufacturer's instructions.

Regardless of the manufacturer, dry scrubbers are relatively similar in design. Few proprietary or patentable features exist, and therefore one would expect that most of these systems would operate at virtually the same high level, rather than provide emissions that vary by more than an order of magnitude. The range of emissions could be attributed to random factors, such as differences in incinerator design, feed rate, waste being burned, operator decisions, testing variables, and other site specific factors. Since incinerator operations do experience wide variations in these conditions, that possibility is credible to a certain point, but not to the point of explaining emissions that vary to the extent that has been reported. 
It would be extremely helpful if definitive test data was available to evaluate the impact of all of these variables on the operations. Unfortunately there is not for a number of reasons:

- Since all of the operational variables do exist, it is very difficult to hold all but one constant and vary individual items while testing for the results.

- The cost of testing to this level would be considerable.

- Medical waste incinerators are relatively small installations, and in recent years they have represented a fairly inactive market. Therefore, they have not received much attention by the major air pollution control companies and trade associations that could fund research and development studies similar to those being done for applications such as utility boilers.

Where the same basic design and operational instructions have been maintained in air pollution control systems, there have been reasonably consistent high removal efficiencies (Table 3 ) from one manufacturer. This paper presents arguments to explain these results. In the absence of a sufficient data base and research, we must present a subjective analysis of these factors. The contribution of each individual factor cannot by quantified, but we can identify that by following these design criteria there have been consistent operating results and these results in general are better than the overall industry has experienced.

The five (5) dry scrubber, medical waste incinerator APC installations cited in Table 3 all were supplied by our company and all have virtually the same design concept and operations. However, the applications include both commercial and on-site medical waste incinerators; fixed hearth and rotary kiln combustors; they have used woven, felted, and membrane bags; and had other detail variations, yet the results have been universally high as indicated.

This paper provides a discussion of the practical, common sense design aspects that have caused this high performance level. Some of the points may seem obvious but they have been overlooked a sufficient number of times to warrant stating them.

\section{Dry Scrubber Operation}

For background we would like to review in general the design and operation of a dry scrubber system and discuss some of the component alternatives.

There are three primary steps in dry scrubbing as shown in Figure 1. First, the gas stream is conditioned to make it suitable to be handled by the downstream equipment. Generally, this consists of cooling the gas from a typical incinerator discharge temperature of about $2000^{\circ} \mathrm{F}$ to that which is consistent with baghouse and other component design, generally less than $450^{\circ} \mathrm{F}$. In addition further temperature reduction and/or humidification may be performed to improve the reactivity of the acid gases and/or the adsorption and condensation of individual pollutants.

The second step is to inject reagents into the gas stream to react with and/or adsorb the pollutants and turn them into solid particulates. Once the reagents are injected they begin to react with the pollutants. The reaction efficiency depends on the reagent and pollutant establishing contact for a sufficient amount of time. Therefore it is critical that the reagent be injected in such a manner that all of the pollutants are contacted. While a part of the removal takes place in the ductwork and any residence chamber, a substantial part of the reaction occurs in the baghouse. The unused reagent builds up in the filter cake on 
the bags where there is intimate contact with the pollutants as the gas stream moves through the filter cake at a relatively low apparent velocity (approximately $3 \mathrm{ft} / \mathrm{min}$ ). The third step of the dry scrubbing process is to remove the solid particulates which now include the particulate matter, reacted acid gases, and adsorbed pollutants.

Depending on the site conditions, customer and vendor preferences, and economics, different alternatives may be used for each of the individual system components as described in Figure 2. In addition to these primary system components, there are sub-systems and auxiliary items that are critical to reliable operation but are not a factor in performance efficiency.

Simplistically, it may seem that a dry scrubber is just a combination of three independent components and that the system is no more interdependent than to require alarms and feedback of the operating status. However, for optimum operation, these components in a dry scrubber system have to be selected, designed, configured, and operated as an integrated system.

\section{Basic Design Criteria}

There are several fairly obvious design criteria (although they are not always adhered to) that are important for system operation, and there are a few perhaps less obvious, but equally critical design and operational criteria that make the difference between barely acceptable performance and excellent results.

Some of the basic design criteria that we would classify as being fairly obvious, include the following:

- Every component of the system has to be designed to allow for gas volume and temperature swings that come with charging the incinerator, especially with a fixed hearth incinerator.

- The exit temperature from the gas cooling device has to be controlled to a narrow range and the control method has to allow for variations in both the inlet temperature and gas volume.

- Regardless of the reagent used, the material's specifications for chemical composition, particle size distribution, purity, and activity are critical.

- Reagent injection must be uniform across the entire gas stream.

- Ductwork must be designed to keep the reagent distribution even across the gas stream, especially at the baghouse inlet.

\section{Unique Design Issues}

There are a couple less obvious design issues, or areas where there have been frequent misapplications. These are in regard to the operation of the reagent feed system and the baghouse design so that it is appropriate to be a part of a reaction system.

\section{Reagent System}

The reagent system typically includes a storage bin or silo, a mechanical flow enhancer (i.e. vibrator, live bin discharger, or paddles), followed by a volumetric feeder and then by one of the delivery methods described in Figure 2 to the point of injection, where it is mixed with the gas stream.

Obviously, maintaining the correct feed rate is important. Initially the feeder is usually calibrated by physically measuring the delivery at two or three settings and pro-rating between these readings. The 
assumption is that the reagent feeder will consistently maintain that feed rate for all time; however, that is not necessarily the case. Operating conditions can change the feed rate. There will inevitably be material build up in the components of a pneumatic conveying system and that build up will restrict flow and add back pressure on the system. Although these systems use an airlock to minimize back pressure on the volumetric feeder, airlocks will always have some leakage. As the pneumatic conveyor's operating pressure increases, the back pressure increases and reagent feed is slowed. This can easily account for a reduction of $25 \%$ or more in the actual reagent feed versus the calibration. To make matters worse, the calibration of the reagent feed rate may have been done without the pneumatic conveying system connected. In this case, the actual flow rate will always be less than expected because of the normal line pressure.

In mechanical systems it is less likely that there will be an undetected feed reduction. If there is build up in a mechanical system it is likely to cause a spillage which would be obvious.

In most reagent feed systems there is a flow enhancer to maintain a "uniform" flow of the reagent (often either a vibrator or live bin discharger). To insure flow into the system, these flow enhancers normally operate intermittently, and they will cause a variation in flow rate. During the period that the flow enhancer is operating, the feed rate can be as much as $50 \%$ higher than when it is not operating. Figure 3 shows how actual flow can vary with different operating periods. Many times the operating periods of the device will be changed to reduce an annoying sound, to help avoid plugging or for some other reason without consideration of its effect on reagent feed. This may have the system operating with a significantly different reagent supply rate than planned.

\section{Baghouse Design}

Proper baghouse design is absolutely crucial for the success of a dry scrubbing system. Unfortunately, the equipment design criteria that has been established for baghouses which function solely as particulate devices are not necessarily applicable when the unit is used as part of a reaction system. The following items are different:

- Traditional baghouses have a baffle plate at the inlet that is designed primarily to knock out large particulate. However, in a dry scrubber system, since a substantial amount of the reaction occurs in the filter cake on the bags, it is critical to maintain the reagent in suspension in the gas stream and evenly distribute it to the bags. Therefore the inlet of a dry scrubber baghouse should be designed to keep particulate in the gas stream rather than remove it. It is still important that a baffling system be used to prevent direct impingement of the particulate on the bags which would cause wear and premature failure. Figure 4 shows one successful method that was established through a physical scale model.

- The proper air-to-cloth ratio for a baghouse has traditionally been accepted as the highest value that can be used (to reduce cost) that will maintain an acceptable pressure drop, provide acceptable bag life, and meet the required outlet emissions. Generally outlet emissions are achieved relatively easily and therefore bag life and pressure drop have become the determining design criteria. However with individual pollutant control, fine particulate control, and the baghouse serving as part of the reaction in a dry scrubber, this is no longer the case. A lower air-to-cloth ratio will improve the removal efficiency of fine particulate (which includes heavy metals). Furthermore, a lower air-to-cloth ratio reduces the velocity of the acid gases as they pass through the filter cake, allowing a longer time for reaction to occur. Also at a lower velocity, the same pressure drop across the filter cake allows the development of a thicker filter cake, again prolonging the reaction opportunity. 
- The development of the filter cake on the bags is important to improve the particulate removal efficiency in the baghouse. A clean bag is a relatively poor filter but a bag with a surface coating of particulate is much more efficient. In conventional baghouses that are used for particulate removal only, the important criteria is just to maintain a relatively loose filter cake on the bag surface. However, in a dry scrubber system there is another important criteria. The filter cake that is developed by the unused reagent will gradually react with the pollutants until the unused reagent base is consumed and is no longer effective as part of a reaction. Therefore it is important that this filter cake be purged and replaced with fresh material on a periodic basis. Since air-to-cloth ratios are generally low in a dry reactor system, pressure drop alone is not necessarily the best design criteria for bag cleaning. Pressure drop can be relatively low and sometimes is maintained without any bag pulsing. The outer layer of dust and ash just shears off during operation or at shut down. The agglomerated filter cake which is reacted is more likely to be retained on the bag's surface in these conditions. Therefore, although the pressure drop seems to be appropriate, there is not the reaction available in the filter cake. In order to avoid this, the filter cake needs to be purged on a periodic basis, regardless of the system pressure drop. This purging needs to be done carefully since complete removal of the filter cake on all bags at one time would provide a period of no effective filter cake at all and would cause substantially reduced $\mathrm{HCl}$ removal efficiency. Bag cleaning by row must be staggered with a sufficient time interval between pulses to assure that there always is a substantial percentage of the baghouse that is efficient in the reaction system. We consider this operation to be called "filter cake management", and it is perhaps the most important new consideration in dry scrubbing baghouse design.

There are other issues that one would have expected to be more important in dry scrubber baghouse systems than has proven to be the case.

\section{Bag Material}

There are three generic fabrics used in baghouses: membrane, felt and woven construction. Each of these materials is available in different fibers that offer varying temperature capability, chemical resistance, and wear factors, as well as price. We would expect all materials within a group (i.e. all felts) to behave similarly. On the other hand, the three different fabrics form substantially different filter cakes and since the filter cake is so important to performance, it would be expected that different results for particulate removal as well as acid gas scrubbing would be experienced. For instance, a membrane bag provides an extremely smooth surface that causes formation of a relatively dense filter cake which is then easily shed during a pulse. It had been feared that this bag would not be efficient in acid gas scrubbing, both because the dense filter cake gives a more rapid increase in pressure drop for a smaller amount of material on the bag, and virtually the entire filter cake could be removed during bag pulsing, giving an interval of no filter cake reaction. Felt materials are regarded as providing the "fluffy" low density filter cake that would be most effective in reaction applications. Woven materials tend to allow some penetration of the filter cake into the depth of the fabric where it is more difficult to purge the filter cake and assure regeneration with unused reagent.

In practice by following filter cake management criteria with some consideration for the different fabric structures all materials have been shown to provide adequate high efficiency $\mathrm{HCl}$ and particulate removal as shown in Table 4. Although there is some correlation between bag material and efficiency. 


\section{Reagent}

Virtually all medical waste incinerator dry scrubbers use either sodium based or lime based reagents. These two reagent types react in a different manner with the acid gas and they are effective at different temperatures. While we might expect to see significant variations in the acid gas scrubbing efficiency between sodium based and lime based reagents, that has not proven to be the case, so long as the system design provides the proper temperature and the correct stoichiometric ratio is used. Sodium based reagents are more effective in the removal of $\mathrm{SO}_{2}$; however, $\mathrm{SO}_{2}$ removal has not been an issue in medical waste incinerators.

\section{Conclusion}

In conclusion, there is little difference in the basic design of dry scrubber systems, and we would expect to see similar performance of all systems. However, there are substantial differences in the operating results, and they seem to depend on some detail design issues and operation of the systems. Optimizing the performance depends on first achieving high efficiency operation and then avoiding periods where reaction efficiency is reduced and/or when not all of the gas stream is effectively controlled. When trying to achieve removal efficiencies in the upper $90 \%$ range, there is no room to allow for even a very small percentage of the gas stream to be untreated or to allow a break down in the effective treatment for a small percentage of the time.

With proper design dry scrubber systems can readily meet the proposed EPA standards for medical waste incinerators and do so consistently and reliably.

\section{References}

1. U.S. Environmental Protection Agency. (Standards of Performance for New Stationary Sources and Emission Guidelines for Existing Sources: Medical Waste Incinerators); RIN 2060-AL62, June 1996.

2. Turner, Mark B.; Hanks, Katie; Midwest Research Institute; Memo to Richard Copeland, U.S. Environmental Protection Agency; Dated May 20, 1996; IV B 049. 


\section{Table 1}

Revised MACT Floor Emission Levels

For New MWI ("Large" incinerator subcatagory)

\section{POLLUTANT}

Particulate matter

CO

Dioxin

$\mathrm{HCl}$

$\mathrm{Pb}$

$\mathrm{Cd}$

$\mathrm{Hg}$
EMISSION LEVEL

$0.015 \mathrm{gr} / \mathrm{dscf}$

$40 \mathrm{ppmdv}$

$25 \mathrm{ng} / \mathrm{dscm}$

15 ppmdv ( $99 \%$ reduction)

$0.07 \mathrm{mg} / \mathrm{dscm}$ ( $98 \%$ reduction)

$0.04 \mathrm{mg} / \mathrm{dscm}$ ( $90 \%$ reduction)

$0.55 \mathrm{mg} / \mathrm{dscm}$ ( $85 \%$ reduction)

Table 2

Dry Scrubber Systems

Range of Emissions from EPA. Test Sites

POLLUTANT

Particulate matter (gT/dscf)

Dioxin (ng/dscm)

$\mathrm{HCl}$ (ppmdv)

(\% reduction)

$\mathrm{Pb}$ (mg/dscm)

$\mathrm{Cd}(\mathrm{mg} / \mathrm{dscm})$

$\mathrm{Hg}$ (mg/dscm)

\section{$\underline{\text { LOW }}$}

0.001

0.5

0.0

$89.1 \%$

0.002

0.001

0.010
HIGH

0.009

19.9

179.8

$100 \%$

0.59

0.028

0.446
RANGE

$9 \mathrm{X}$

$40 x$

nc

nc

$30 \mathrm{X}$

$28 X$

$45 X$ 


\section{Table 3}

One Manufacturer's Dry Scrubber Emission

Results versus EPA'S Data (PM \& $\mathrm{HCl}$ )

\section{INSTALLATION}

Alabama

Florida, site \#1

Florida, site $\$ 2$

Michigan

New York

Average

Range

"Achievable" per EPA

\section{PMEMISSION}

$0.0031 \mathrm{gr} / \mathrm{dscf}$

$0.0024 \mathrm{gr} / \mathrm{dscf}$

$0.0003 \mathrm{gr} / \mathrm{dscf}$

$0.0017 \mathrm{gr} / \mathrm{dscf}$

$0.0009 \mathrm{gr} / \mathrm{dscf}$

$0.0017 \mathrm{gr} / \mathrm{dscf}$

$+1-0.0014$

$0.015 \mathrm{gr} / \mathrm{dscf}$
HCl REMOVAL

$98.1 \%$ ( 9 ppmdv)

$97 \%$ (12.5 ppmdv)

$99.75 \%$ (1.3 ppmdv)

$98 \%$ (12 ppmdv)

$99.8 \%$ (2.6 ppmdv)

$98.5 \%(7.5 \mathrm{ppmdv})$

$+1.3 /-1.5 \%$

$+5 /-6.2 \mathrm{ppmdv}$

$93 \%(100 \mathrm{ppmdv})$

Table 4

Efficiency versus Fabric Type

FABRIC TYPE

Woven fabric

Membrane

Felted fabric
AVERAGE PM EMISSIONS

$0.0024 \mathrm{gr} / \mathrm{dscf}$

$0.0017 \mathrm{gr} / \mathrm{dscf}$

$0.0009 \mathrm{gr} / \mathrm{dscf}$
AVERAGE HCl

REMOVAL

$97 \%$

$98.6 \%$

$99.8 \%$ 


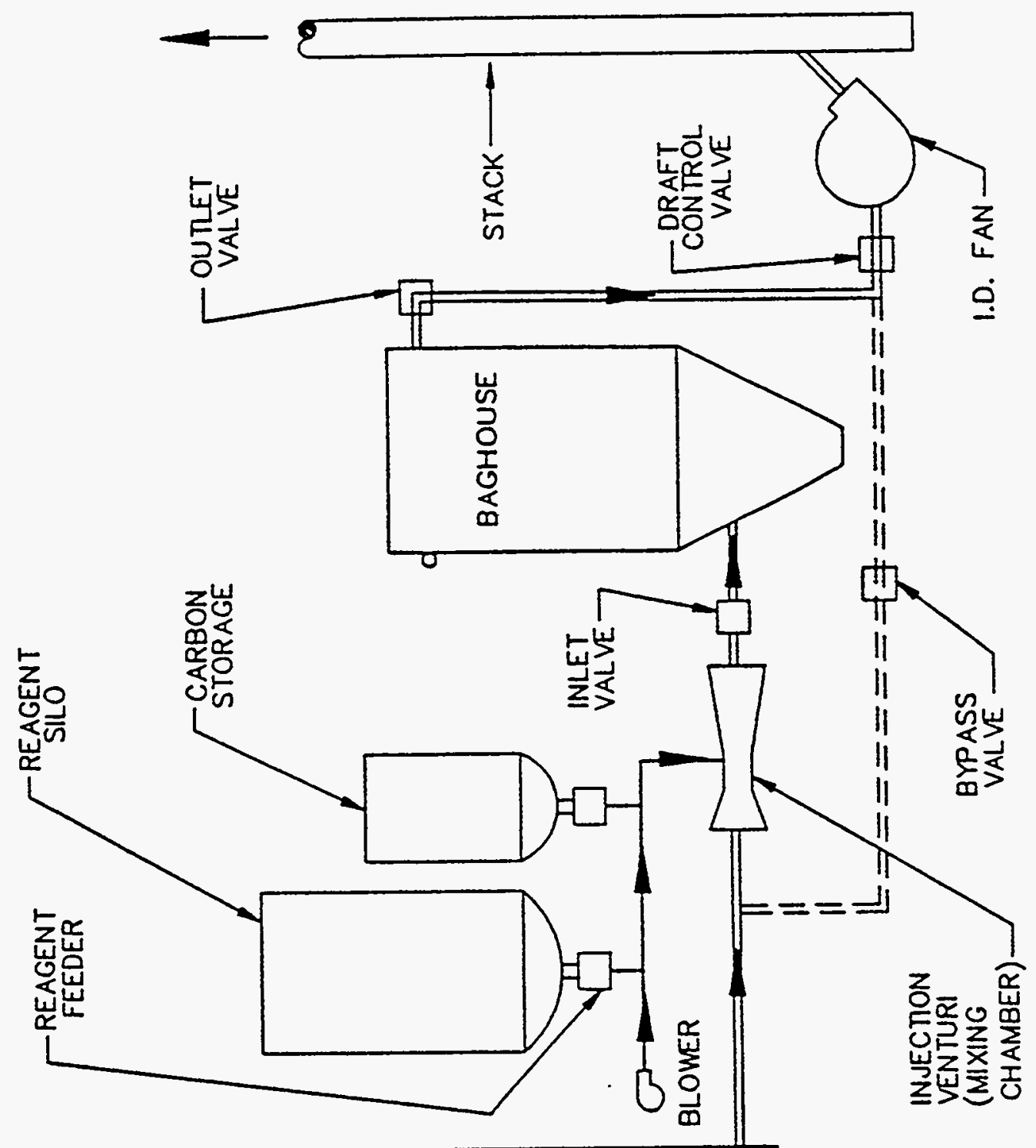

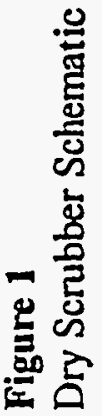

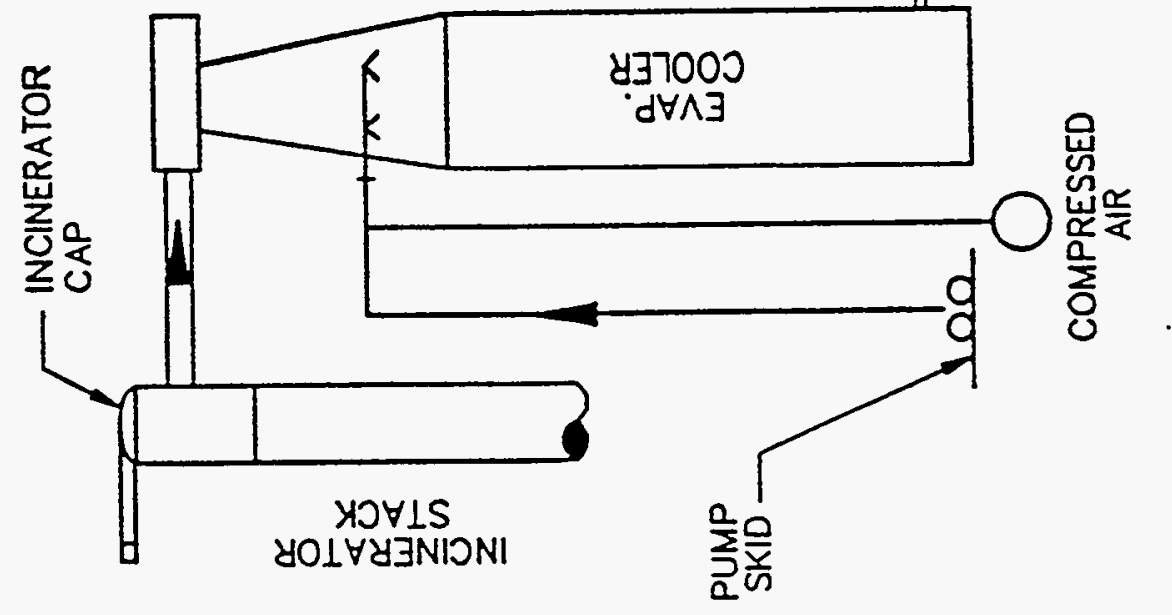




\section{Figure 2}

Dry Scrubber Component Alternatives

GAS COOLING: Heat recovery boiler

Water evaporation (evaporative cooler)

Dilution air (air quench/tempering air)

Heat exchanger

Combinations (i.e. boiler plus heat exchanger)

REAGENTS:

Calcium based (Hydrated lime)

Sodium based (Sodium bicarbonate, Trona, etc.)

Activated carbon (adsorbent, not for $\mathrm{HCl}$ )

REAGENT INJECTION: Dry injection (gravity, mechanical, or pneumatic)

Spray dryer

SOLIDS REMOVAL: $\quad$ Pulse jet baghouse (most common)

Reverse air or shaker baghouse

Electrostatic precipitator 


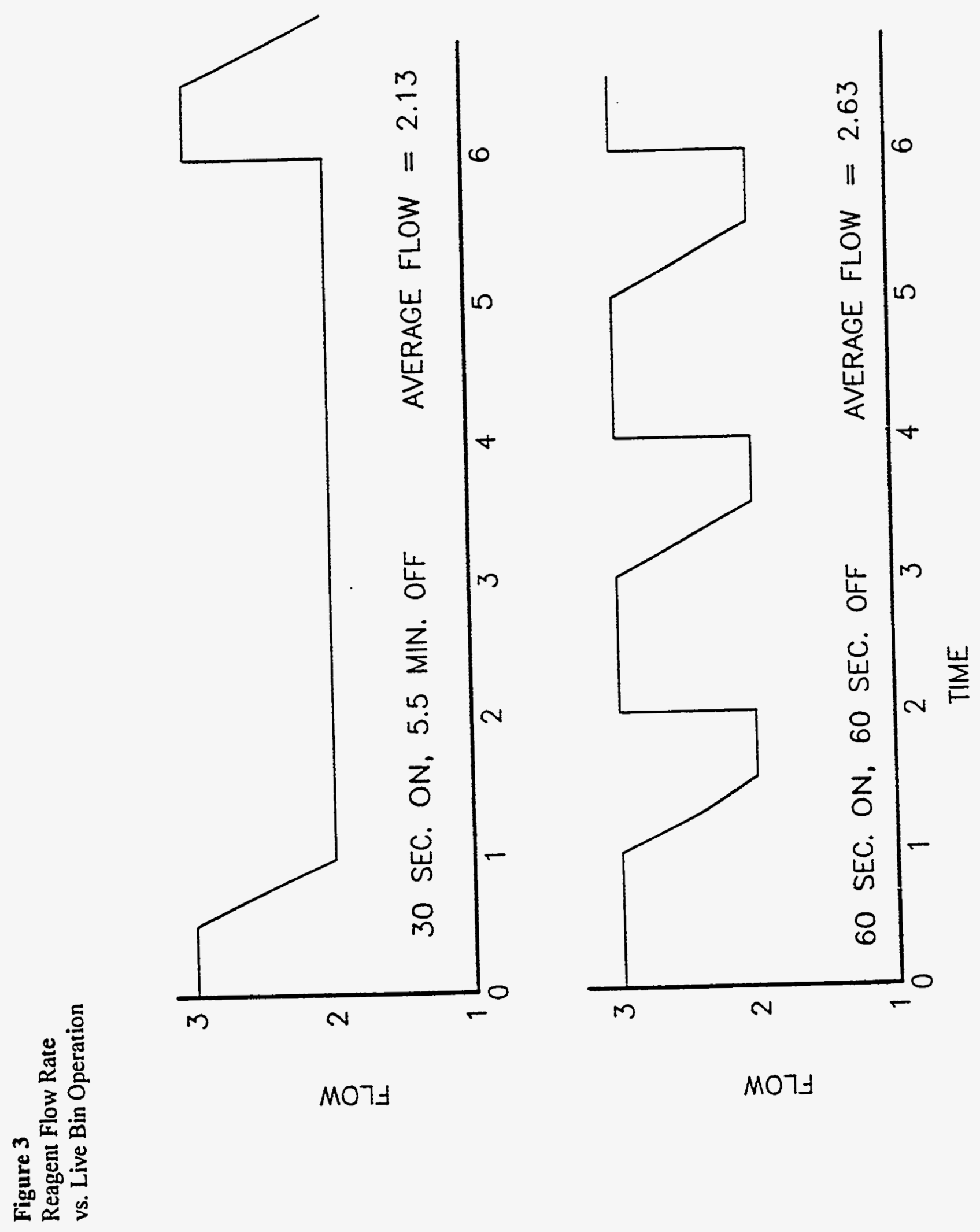


Figure 4

Baghouse Inlet Distribution

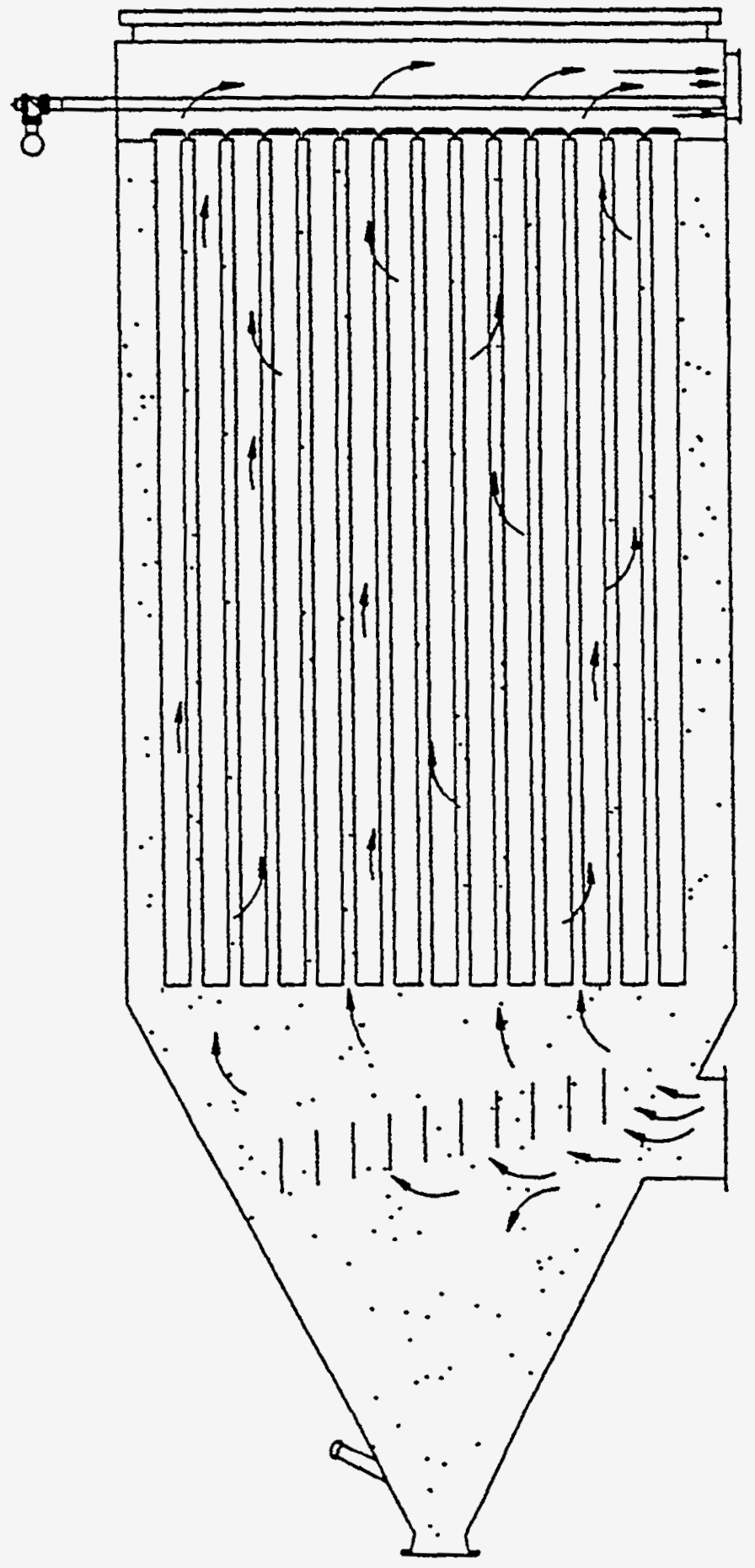




\section{FILTRATION LAMINATE TECHNICAL DATA:}

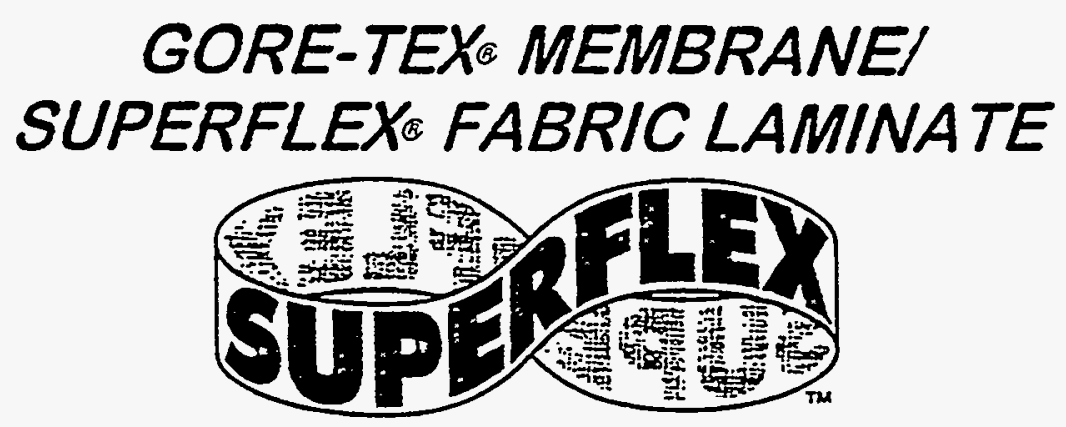

Fabric Construction:

Modified Crowfoot

Fiber Content:

Fluoropolymer-Fiberglass

Composite

Continuous Operating Temperature:

$500^{\circ} \mathrm{F}\left(260^{\circ} \mathrm{C}\right)$

Maximum Surge Temperature:

$550^{\circ} \mathrm{F}\left(288^{\circ} \mathrm{C}\right)$

Acid Resistance:

Very Good

Alkali Resistance:

Fair

Weight:

20 oz/yd ${ }^{2}$

Thickness:

$0.034^{\prime \prime}$

Breaking Strength:

Machine Direction:

Cross Machine Direction:

Mullen Burst:
$350 \mathrm{lbs} / 1$ " Ravel

400 lbs/1" Ravel

900 psi
MIT Fiex:
Over $1,000,000$ cycles

All data expressed as typical values.

(D) SUPERFLEX and the SUPERFLEX symbol are registered trademarks of W. L Gore \& Associates, Inc.

Patent Number $5,456,983$

Rev. 5/20/93: $1 / 96$

This document is considered confidential and proprietary. It is intended only for the use of those employed by, and/or customers of, W. L. Gore \& Associates, Inc.

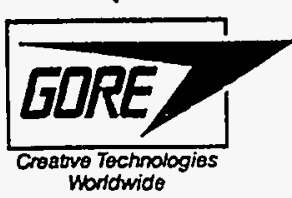

W. L. Gore \& Associates, Inc.

101 Lewisville Rd., P.O. Box 1100, Elkton, MD 21922-1100

Phone: 410-392-3300 Fax: 410-398-6624

GORE-TEX is a registered trademark of W. I. Gore \& Associates, Inc. 


\section{PARTICULATE MATTER EMISSIONS}

\section{GORE-TEX® MEMBRANE FILTER BAGS}

\begin{tabular}{|c|c|}
\hline Facility Description & gr/dscf @ 7\% $\mathrm{O}_{2}$ \\
\hline \hline Municipal Waste Combustor & 0.0004 \\
\hline Municipal Waste Combustor \\
Unit 1 \\
Unit 2 \\
Unit 3 & 0.0005 \\
Unicipal Waste Combustor & 0.0006 \\
Unit 1 & 0.0007 \\
Unit 2 & 0.0007 \\
\hline \hline Whole Tire Incinerator & 0.0004 \\
\hline Fluid Bed Tire Incinerator & 0.001 \\
\hline Medical Waste Incinerator & 0.0007 \\
\hline Medical Waste Incinerator & 0.001 \\
\hline \hline Medical Waste Incinerator & 0.001 \\
\hline Soil Remediation & 0.001 \\
\hline \hline Hazardous Waste Incinerator & 0.0008 \\
\hline \hline
\end{tabular}

PM emission results are for front-half catch only, as defined by U.S. EPA Method 5, or equivalent. 


\section{GORE-TEX ${ }^{\circledR}$ Membrane Filter Bags}

\section{Incinerator Applications Highlights}

\begin{tabular}{|c|c|}
\hline Application & Benefits \\
\hline $\begin{array}{l}\text { Municipal Solid Waste: } \\
\text { Four separate boiler lines. Air pollution control } \\
\text { equipment employs S.D.A. with pulse jet fabric } \\
\text { filters. GORE-TEX }{ }^{\circledR} \text { membrane filter bags installed } \\
\text { and evaluated over many years. }\end{array}$ & $\begin{array}{l}\text { Lower Emissions; lower pressure differential; } \\
\text { extended filter bag life; cost justifications based on } \\
\text { operating data. }\end{array}$ \\
\hline $\begin{array}{l}\text { Paper Mill Sludge: } \\
\text { Existing system using off-line pulse jet baghouse. } \\
\text { Conventional media had emissions and blinding } \\
\text { problems. }\end{array}$ & $\begin{array}{l}\text { Lower emissions and pressure differentials; above } \\
\text { design combustion capacity; increased bag life due } \\
\text { to W. L. Gore \& Associates, Inc.'s recommended } \\
\text { system modifications. }\end{array}$ \\
\hline $\begin{array}{l}\text { Hospital Waste: } \\
\text { New system, on-line pulse jet; no acid gas } \\
\text { conditioning. }\end{array}$ & $\begin{array}{l}\text { Greater than three years bag life. } \\
\text { Enhanced } \mathrm{HCl} \text { scrubbing. }\end{array}$ \\
\hline $\begin{array}{l}\text { Thermal Decontamination: } \\
\text { Existing systems; on-line puise jet; conventional } \\
\text { media-short bag life and high pressure differential }\end{array}$ & $\begin{array}{l}\text { Lower emissions; two year average bag life; lower } \\
\text { differential pressure resulting in design gas } \\
\text { volume. }\end{array}$ \\
\hline $\begin{array}{l}\text { Waste Solvents: } \\
\text { Existing on-line pulse jet. Conventional media } \\
\text { caused frequent shutdowns to maintain the wet } \\
\text { scrubber; less than six months bag life. }\end{array}$ & $\begin{array}{l}\text { Reduction in particulate emissions; extended bag } \\
\text { life; lower pressure differentials. }\end{array}$ \\
\hline $\begin{array}{l}\text { Waste Oil and Sludge Filtrate: } \\
\text { Replacement of existing APC system; existing } \\
\text { system had both pressure differential and emission } \\
\text { problems. }\end{array}$ & $\begin{array}{l}\text { W. L. Gore \& Associates, Inc. consulted in new } \\
\text { baghouse design; GORE-IEX }{ }^{\circledR} \text { membrane filter } \\
\text { bags survived process upsets; low pressure } \\
\text { differential; low emissions; extended bag life. }\end{array}$ \\
\hline $\begin{array}{l}\text { Refuse Derived Fuel: } \\
\text { Existing system; on-line pulse jet; conventional } \\
\text { media-high pressure differential resulting in poor } \\
\text { ventilation of mechanically conveyed/transferred } \\
\text { RDF fuel. }\end{array}$ & $\begin{array}{l}\text { Lower operating pressure differentials improved } \\
\text { ventilation; reduced housekeeping and } \\
\text { maintenance; filter bag life extended. }\end{array}$ \\
\hline
\end{tabular}

/ab/thp:incin.doc

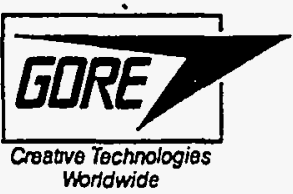

W. L. Gore \& Associates, Inc.

101 Lewisville Rd., P.O. Box 1100, Elkton, MD 21922-1100

Phone: 410-392.3300 Fax: 410-398-6624

GORE-TEX is a registered trademark of W. L. Gore \& Associates, Inc. 


\title{
FILTRATION LAMINATE TECHNICAL DATA:
}

\author{
GORE-TEX ${ }^{\circledR}$ MEMBRANE/ \\ TEFLON ${ }^{\circledR}$ B FIBERGLASS FABRIC
}

Fabric Construction:

Fiber Content:

Continuous Operating Temperature:

Maximum Surge Temperature:

Acid Resistance:

Alkali Resistance:

Weight:

Breaking Strength:

Machine Direction:

Cross Machine Direction:

Mullen Burst:

Thickness:
Modified Crowfoot

ECDE Fiberglass

$500^{\circ} \mathrm{F}\left(260^{\circ} \mathrm{C}\right)$

$550^{\circ} \mathrm{F}\left(288^{\circ} \mathrm{C}\right)$

Very Good

Fair

$16.8 \mathrm{oz} / \mathrm{yd}^{2}$

290 lbs/1" Ravel

$225 \mathrm{lbs} / 1$ " Ravel

600 psi

$0.028^{n}$

All data expressed as typical values.

Revised: May 16, 1991

(i) Teflon is a registered trademark of E. I. du Pont de Nemours \& Co., inc

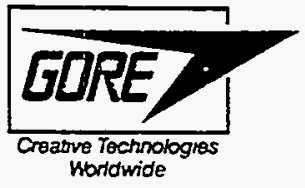

W. L. Gore \& Associates, Inc.

101 Lewisville Rd., P.O. Box 1100, Elkton, MD 21922-1100

Phone: 410-392-3300 Fax: 410-398-6624

GORE-TEX is a registered trademark of W. L. Gore \& Associates, Inc.

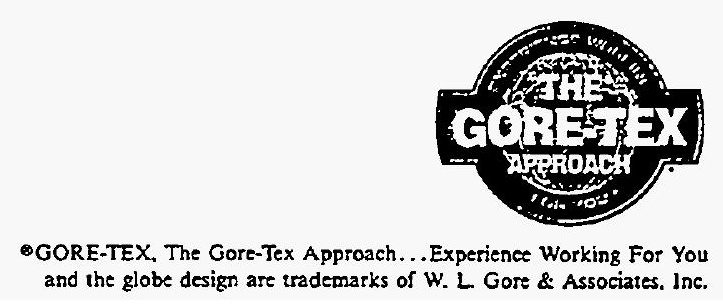
and the globe design are trademarks of W. L Gore \& Associates. Inc. 
NUCON International, Inc.

\section{Design \& Performance Characteristics of MERSORB ${ }^{\circledR}$ Mercury Adsorbents in Liquids and Gases}

NUCON International, Inc.

7000 Huntley Rd., Columbus, OH 43229

Telephone: (800) 992-5192

Fax: (614) 431-0858 


\section{Table of Contents}

Summary

Page

Introduction

Physical Properties

Control Methods

Gas Pbase Applications

Long Term Laboratory Tests 5

Adsorption Capacity

Particle Size Effects

Performance

Pressure Drop

Velociry Effects

12

Temperature Effects

Liquid Phase Applications 15

Mercury Removal From Water $\quad 15$

Adsorption Capacity 15

Effect of Acidity 16

Effect of Residence Time $\quad 17$

Effect of Carbon Substrate $\quad 17$

Oak Ridge National Laboratory Studies 18

Mercury Removal from Hydrocarbon Liquids $\quad 19$

Case Histories $\quad 20$

Water from Air Scrubbers $\quad 20$

Mercury Cell Hydrogen $\quad 20$

Natural Gas Processing 20

Mine Atmosphere 20

Mercury Waste Remediation $\quad 21$

Mercury Removal from Waste Water 21

Fluorescent Lamp Recycling $\quad 21$

$\begin{array}{ll}\text { Operating Guidelines } & 22\end{array}$

$\begin{array}{lr}\text { Technical Support } & 23\end{array}$

$\begin{array}{ll}\text { References } & 24\end{array}$ 


\section{SUMMARY}

Mercury is an historically important and useful industrial material. Mercury and mercury compounds have been used for thousands of years as pigments in inks (cinnabar, red sulphide), aids to early metallurgy (gilding copper), and instrumentation (thermometers, barometers). It is present throughout the earth. However, because it is toxic, human ingestion and exposure must be prevented. When present in industrial fluids that are being processed, mercury causes corrosion and should be removed to prolong the life of industrial equipment.

Mercury has low vapor pressure and low solubility in liquids. Therefore, any mercury removal process must be effective at low concentrations. Adsorption is such a process. Activated carbon is a fair adsorbent for mercury, but its capacity is significantly increased by impregnation with a material that chemically reacts with, and holds, the mercury. The choice of impregnant is dictated by the composition of the fluid. Sizing of adsorption equipment is determined by the flow rate of the fluid stream and the desired life of the adsorbent.

This bulletin describes NUCON products and processes for control of mercury and its compounds. 
NUCON 11B28 R1 - 10/97

\section{INTRODUCTION}

Mercury is used in many industrial processes and products. It is used as the cathode in the generation of chlorine by electrolysis of sodium chloride. It is also used to manufacture batteries, catalysts, specialty chemicals, and fungicides. Electronics manufacturers use mercury for switches and measuring instruments. Mercury is present in fluorescent lamps.

Petroleum products contain mercury. A number of tests have been made to determine the concentration of mercury in natural gas supplies in various parts of the U.S. Locations in South Texas have shown high concentrations, reported to range from $.002 \mathrm{mg} / \mathrm{m}^{3}$ to over $4.5 \mathrm{mg} / \mathrm{m}^{3}$. ${ }^{(1)}$ Mercury is also present in "condensate" from other parts of the world such as Indonesia and North Africa.

The exhaust gas from waste incinerators and coal burning power plants contains mercury. It is estimated that half of the global emissions of mercury come from fossil fuel combustion. Although the total quantity emitted by waste incinerators is less, the concentrations are much higher and, therefore, potentially more toxic.

Waste spillage containing mercury has resulted in contamination of soil and water. Waste materials containing mercury are sometimes stored in landfills that are not completely isolated from the surrounding environment.

Mercury is considered hazardous because of its toxicity. The Threshold Limit Values-Time Weighted Average (TLV-TWA), established by AIGCH, is $0.05 \mathrm{mg}$ mercury per cubic meter air. ${ }^{(2)}$ The typical concentration of mercury found in urban air is 7 nanograms per cubic meter. ${ }^{(3)}$ (In remote and rural areas it is approximately $10 \%$ of that level). These levels are considered harmless because they are 10 million times less than the TLV. However, in some industrial environments, concentrations as high as $5 \mathrm{mg}$ per cubic meter of air have been measured. This level is 100 times the TLV.

Mercury can amalgamate with metals used in process equipment causing corrosion and failure. It is a poison for some catalysts used in hydrocarbon processing.

In order to protect the health of humans and prolong the life of industrial equipment and materials, it is necessary to remove mercury from various air, gas and fluid streams. 


\section{Physical Properties}

General physical properties are shown in Table 1.

\section{Table 1. Physical Properties of Mercury}

\author{
Solubility in water \\ Saturation concentration, $20^{\circ} \mathrm{C}$ \\ Melting point \\ Boiling point \\ Density
}

$0.064 \mathrm{mg}$ per liter $r^{(4)}$

$20 \mathrm{mg}$ per cubic meter air

$-38.9^{\circ} \mathrm{C}$

$356.6^{\circ} \mathrm{C}$

$13.5 \mathrm{~g}$ per $\mathrm{ml}$

Mercury is the only metallic element that is liquid at room temperature. Its low vapor pressure makes it useful in applications such as thermometers and manometers. The combination of liquid state and good electrical conductivity makes it a good material for electronic switching devices.

The physical solubility of mercury in hydrocarbon liquids at room temperature is shown in Table $2 .{ }^{(5)}$

\section{Table 2. Solubility of Mercury in Organic Liquids, mg/liter}

$\begin{array}{ll}\text { Heptane } & 1.3 \\ \text { Benzene } & 2.4 \\ \text { Iso Octane } & 0.8 \\ \text { Isopropyl Ether } & 1.0\end{array}$

Typically, the solubility of mercury in hydrocarbons is ten times greater than in water. Since some geologic formations contain both liquid mercury and hydrocarbons, the natural gas and hydrocarbon liquids recovered can have very high mercury contents. 


\section{Control Methods}

Most control techniques employ adsorbents (plain or impregnated) in some form. The high surface area of the adsorbents attracts the mercury and facilitates physical adsorption or chemical reaction. The most common base material is activated carbon. Impregnants are chosen for suitability in a particular environment.

NUCON International, Inc. (NUCON) has developed the MERSORB ${ }^{\circledR}$ family of adsorbents for almost every type of mercury removal application.

For processing natural gas, hydrocarbon liquids and small air streams, fixed beds of pelleted MERSORB ${ }^{\circledR}$ adsorbents are used. Even though the adsorbents are optimized for maximum mass transfer rates, the relatively slow reaction rate of the mercury vapor with the impregnant requires a relatively long residence time. The amount of adsorbent required to achieve high removal efficiency will generally give a very long service life.

When the mercury is present at very low concentrations in relatively large gas streams (such as effluent gases from coal fired power plants or waste incinerators), powdered adsorbents can be used. The powdered adsorbents can be injected into the gas stream and, after an appropriate residence time, filtered out in a dust collector. Tests performed have shown various degrees of effectiveness.

MERSORB ${ }^{\circledR}$ is a registered trademark of NUCON International, Inc. 


\section{GAS PHASE APPLICATIONS}

MERSORB ${ }^{\circledR}$ adsorbents are particularly suited to remove mercury from gas streams to protect catalyst beds or aluminum heat exchangers from mercury contamination and corrosion. MERSORB ${ }^{\circledR}$ mercury removal products are specially prepared adsorbents with optimum volumetric efficiency and low pressure drop.

The rate of mercury removal by impregnated carbons is controlled by various diffusion processes. Bulk diffusion to the surface of the particle, pore diffusion, and reactant and reaction-product diffusion in the deposited sulfur layer all affect performance. NUCON has selected adsorbents with optimized pore structure to properly accommodate both the impregnant and the process steps involved in mercury removal.

\section{Long-Term Laboratory Tests}

Mercury removal efficiency and adsorption capacity have been determined in the NUCON laboratory using radioactive mercury isotope.

The relevant test parameters were:

Temperature:

Bed Diameter:

Bed Depth:

Particle Size:

Inlet Concentration:

Pressure:

Linear Velocity: $30^{\circ} \mathrm{C}$

$25 \mathrm{~mm}$

$152 \mathrm{~mm}$

$3 \mathrm{~mm}$ pellets

$32 \mathrm{mg} \mathrm{Hg} . / \mathrm{m}^{3}$ air

$1.0 \mathrm{ATM}$

$3 \mathrm{ft} . / \mathrm{min}$. 
The tests were conducted using six bed segments, each being $25 \mathrm{~mm}$ deep and $25 \mathrm{~mm}$ diameter. The radioactive isotope content of the samples of gas between the segments was analyzed at periodic intervals. The results of the tests for mercury removal from air are shown in Figure 1.

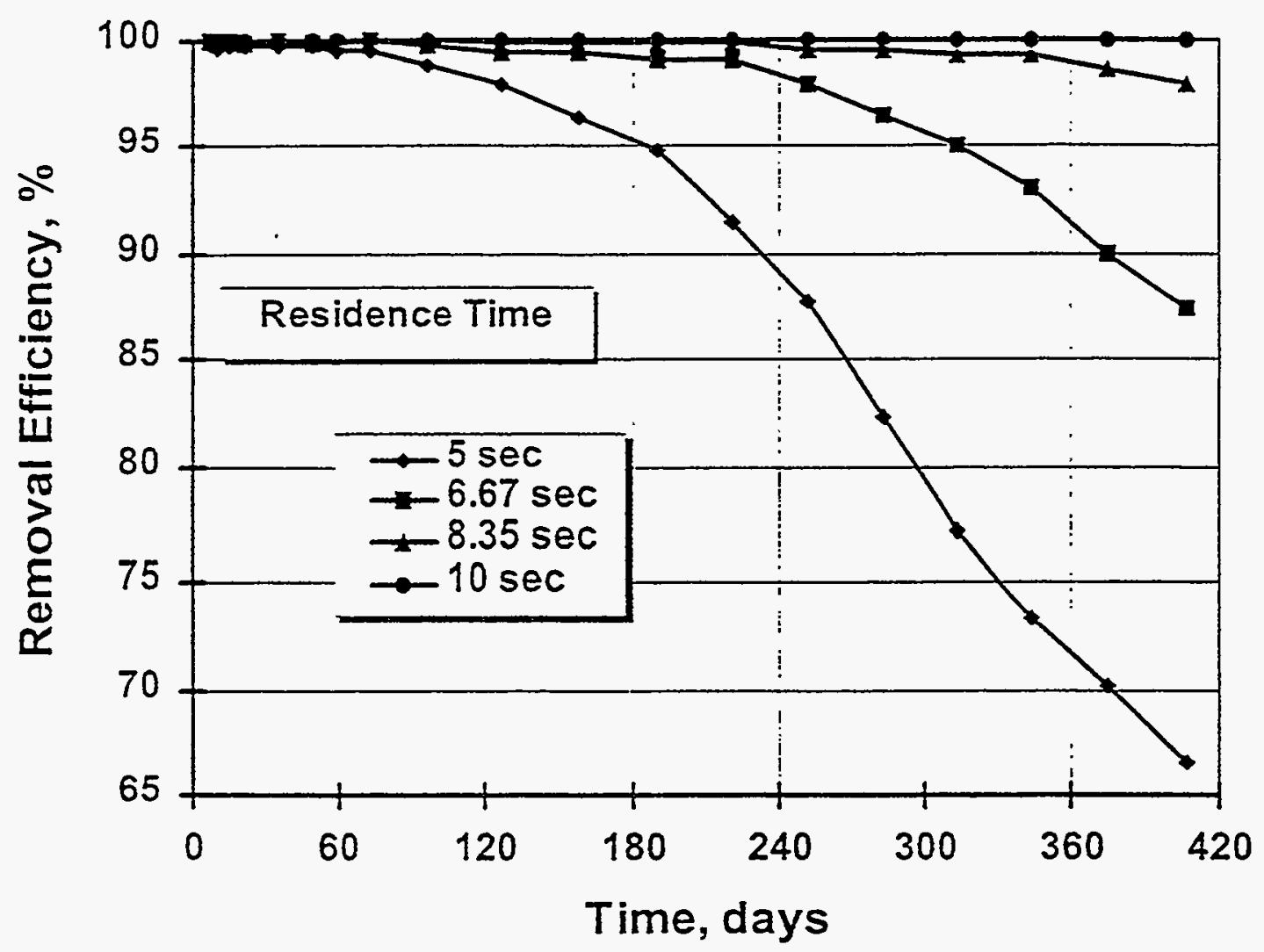

Figure 1 - Mercury removal efficiency from air at various residence times. 
The mercury removal efficiency from natural gas has also been determined. The relevant test parameters were the same as in the air tests. The results are shown in Figure 2.

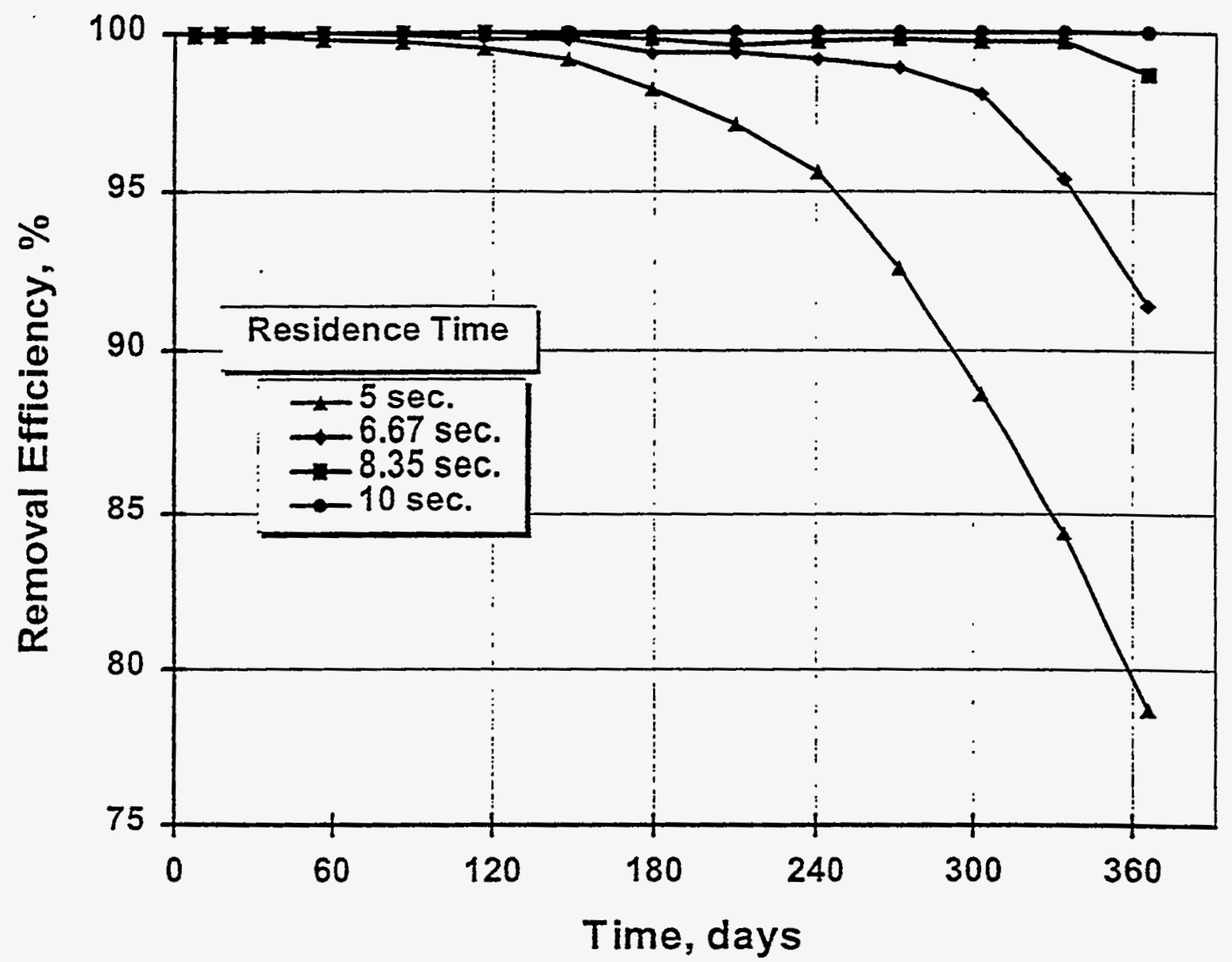

Figure 2 - Mercury removal efficiency from natural gas at various residence times.

For both air and natural gas, a 10-second residence time is recommended to achieve complete removal of the mercury for reasonable lengths of time at high concentration conditions. At these high concentrations, MERSORB ${ }^{\circledR}$ adsorbent removed $100 \%$ of the mercury for over one year. In most commercial applications the mercury concentration is only a fraction of the saturation level, and the life of the MERSORB ${ }^{\circledR}$ adsorbent is typically several years. An alternate approach can be used if removal efficiencies of less than 100\% are acceptable. A smaller bed will give adequate performance for a slightly shorter period of time. For example, a 5 second residence time provided 240 days life at efficiencies above $95 \%$ in the natural gas tests (Figure 2). Similarly, - at low mercury concentrations, $100 \%$ removal can be achieved at less than 10 seconds residence time. 
NUCON 11B28 R1 - 10/97

\section{Adsorption Capacity}

The theoretical equilibrium adsorption capacity of MERSORB ${ }^{\otimes}$ pellets is $85 \mathrm{~g}$ mercury per 100 g MERSORB adsorbent. However, it is impractical to reach that level in commercial applications. An extremely long time would be required to obtain diffusion of the mercury into the pellets and for the chemical conversion to take place. In the region of the mass transfer zone, the amount adsorbed is always less than maximum. Dynamic adsorption capacity data for the extended dynamic adsorption tests are shown in Table 3 . The mercury content of the test bed segments used to generate the data in Figures 1 and 2 was measured by laboratory techniques.

\section{Table 3. Dynamic Adsorption Capacity of MERSORB® $3 \mathrm{~mm}$ Pellets}

$\begin{array}{ccr} & \text { Air } & \text { Natural } \\ \text { Test Duration, days } & 407 & 365 \\ & & \\ & & \\ \text { Bed Segment No. } & & \\ 1 & 23 & 31 \\ 2 & 19 & 28 \\ 3 & 15 & 19 \\ 4 & 15 & 14 \\ 5 & 14 & 12 \\ 6 & 0.3 & 0.3\end{array}$

While Bed Segment No. 6 adsorbed a small amount of mercury, there was no detectable breakthrough from the bed at the end of the test. 


\section{Particle Size Effects}

The particle size of the MERSORB ${ }^{\otimes}$ adsorbent affects several operating parameters. Data concerning the two most important, performance and pressure drop, has been developed.

\section{Performance}

The dynamic performance of adsorbents with small particle size is always better than for larger sizes. Figure 3 shows the difference in performance between MERSORB ${ }^{\circledR} 3 \mathrm{~mm}$ and $4 \mathrm{~mm}$ pellets. These tests were conducted using air saturated with mercury at $30^{\circ} \mathrm{C}$. The test bed dimensions were $25 \mathrm{~mm}$ diameter by $25 \mathrm{~mm}$ long.

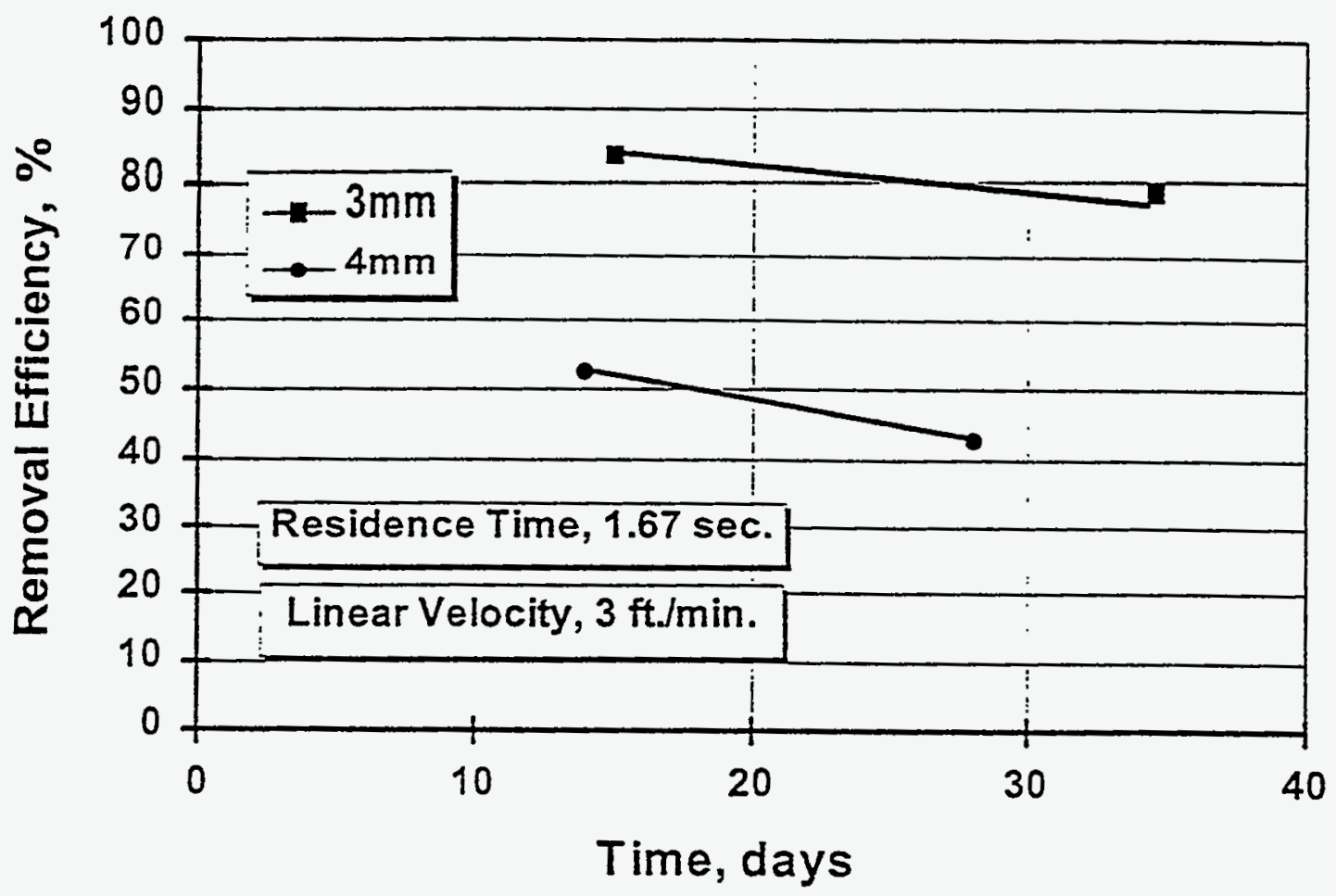

Figure 3 - Effect of particle size on mercury removal from air.

The difference is striking at short residence times. The initial efficiency for $3 \mathrm{~mm}$ pellets at 1.67 seconds residence time is almost $90 \%$, while for $4 \mathrm{~mm}$ it is around $60 \%$. 
Another series of tests was run at twice the flow rate but the same bed geometry. The maximum residence time was five seconds. The linear velocity was higher, which produced more turbulent flow, thereby improving the performance at equal residence times. For example, the efficiency for $3 \mathrm{~mm}$ pellets at 1.67 seconds residence time was $92 \%$ compared to $80 \%$ in the previous test (see Figure 4). This data also shows the performance improvement by using $1.5 \mathrm{~mm}$ pellets. An initial efficiency of $100 \%$ is achieved at a very short residence time of 1.67 seconds. If the additional pressure drop associated with the smaller particle size can be tolerated, the $1.5 \mathrm{~mm}$ product should be used.

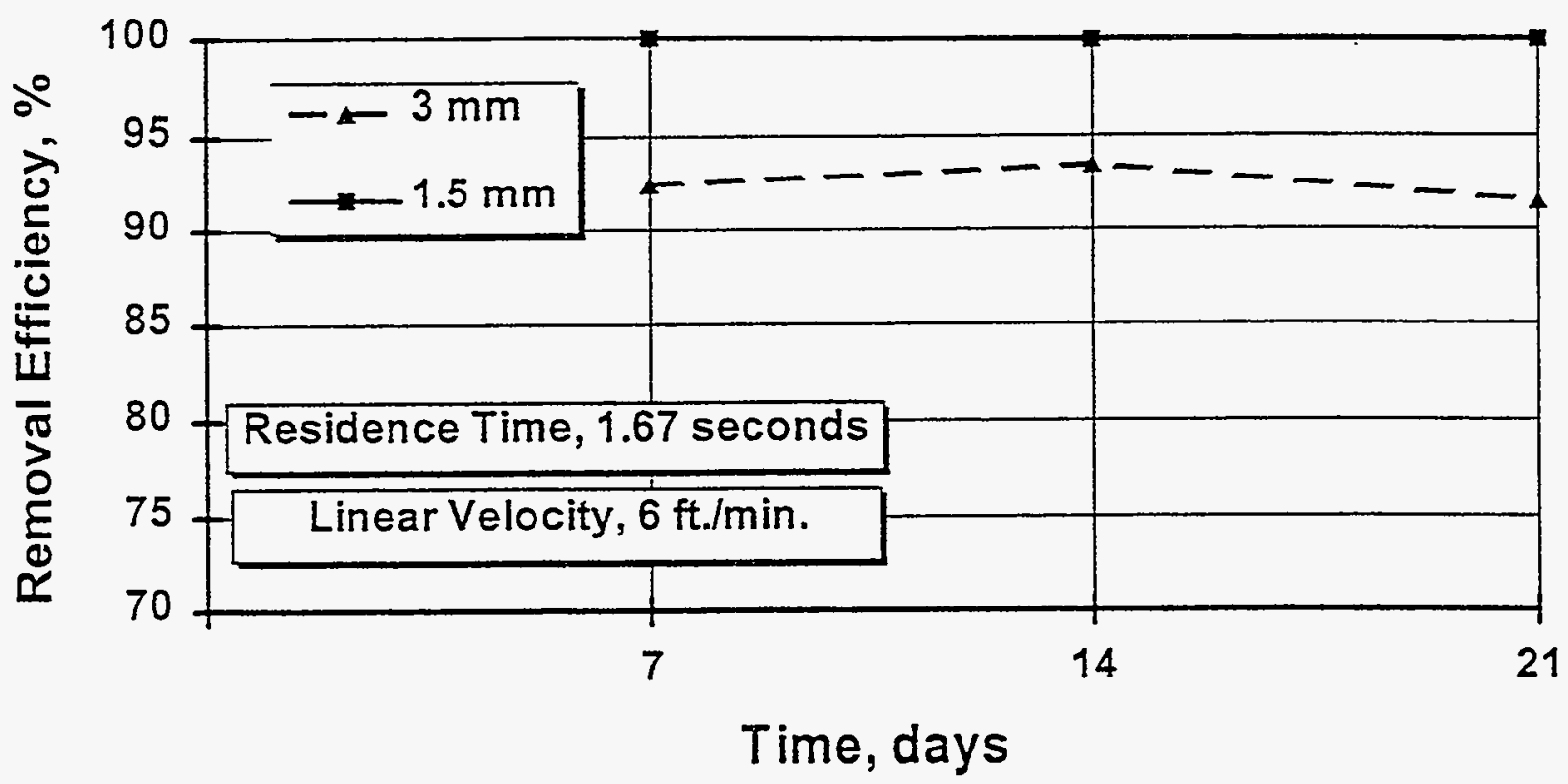

Figure 4 - Effect of particle size on mercury removal from air.

The performance of all three pellet sizes for removal of mercury from air at $6 \mathrm{ft} / \mathrm{min}$. linear velocity and 1.67 seconds residence time is shown in Table 4.

Table 4. Effect of Pellet Size on Performance, 1.67 Seconds Residence Time

$\begin{array}{lrrr}\text { Pellet Diameter, mm } & 1.5 & 3 & 4 \\ \text { Initial Efficiency, \% } & 100.0 & 92.4 & 76.1\end{array}$




\section{Pressure Drop}

The pressure drop through a packed bed increases as particle size decreases. Pressure drop curves for MERSORB ${ }^{\otimes}$ pellets at atmospheric pressure are shown in Figure 5.

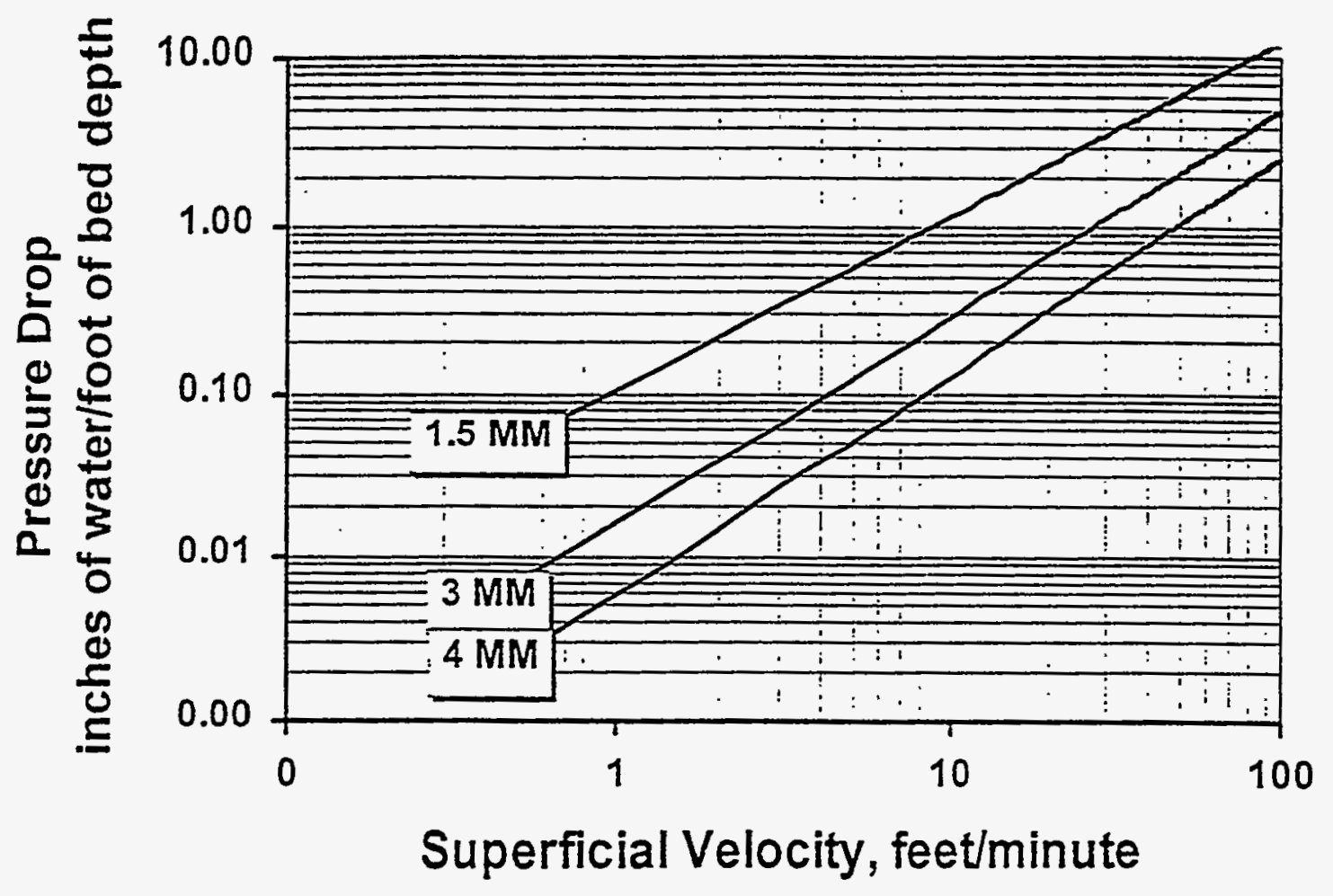

Figure 5 - Pressure drop of air through packed beds.

Natural gas processing is normally done at high pressure. Flow resistance for a typical operating pressure is shown in Figure 6. 


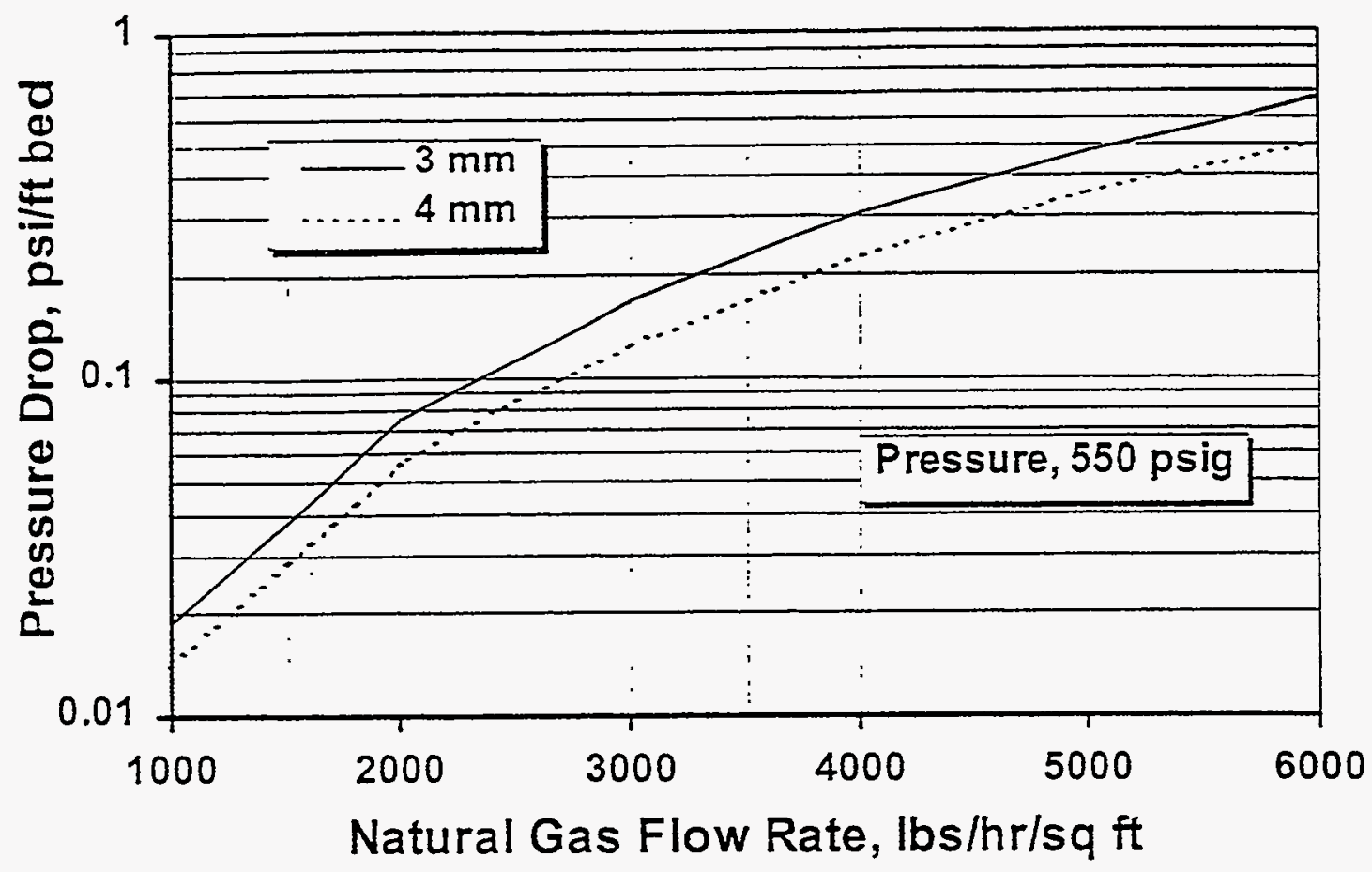

Figure 6 - Pressure drop through MERSORB® pelleted adsorbent.

This curve confirms the low pressure drop resulting from use of pelleted material. For example, the $3 \mathrm{~mm}$ pellets have a pressure drop $50 \%$ below that of a $4 / 10$ granular material.

\section{Velocity Effects}

NUCON ran laboratory tests on $4 \mathrm{~mm}$ MERSORB ${ }^{\circledR}$ pellets using two different gas velocities with the same length bed. The comparative results after 30 days of testing are shown in Table 5 .

\section{Table 5. Effect of Velocity on Dynamic Adsorption}

$$
\% \text { Removal Efficiency }
$$

$\begin{array}{ccr}\text { Residence Time, sec } & 3 \mathrm{ft} / \mathrm{min} & 6 \mathrm{ft} / \mathrm{min} \\ 1.67 & 42.8 & 58.4 \\ 3.33 & 80.3 & 88.7 \\ 5.00 & 90.7 & 100.0\end{array}$

Removal efficiency is generally a function of the residence time. However, at higher superficial - gas velocity, the removal efficiency at a given residence time improves due to favorable diffusion effects. 


\section{Temperature Effects}

Operation of mercury adsorbents at high temperatures is sometimes necessary. There are two major impacts upon the effectiveness of the adsorbents at elevated temperatures. The sulfur impregnant can vaporize in inert atmospheres and can oxidize in air atmospheres.

NUCON uses a unique manufacturing method to make the MERSORB ${ }^{\circledR}$ sulfur-impregnated adsorbents. The result is a material which retains the impregnant better at high operating temperatures than the adsorbents manufactured by others. Thermogravimetric analysis of the NUCON and competitive materials has proven this fact.

The results of thermogravimetric analysis of samples of NUCON $3 \mathrm{~mm}$ material and a competitive 4/10 granular adsorbent are shown in Figure 7. For the competitive material, almost half of the impregnant was lost at temperatures around the boiling point of water. On the other hand, the NUCON material suffers no weight loss until the temperature is higher than $200^{\circ} \mathrm{C}$.

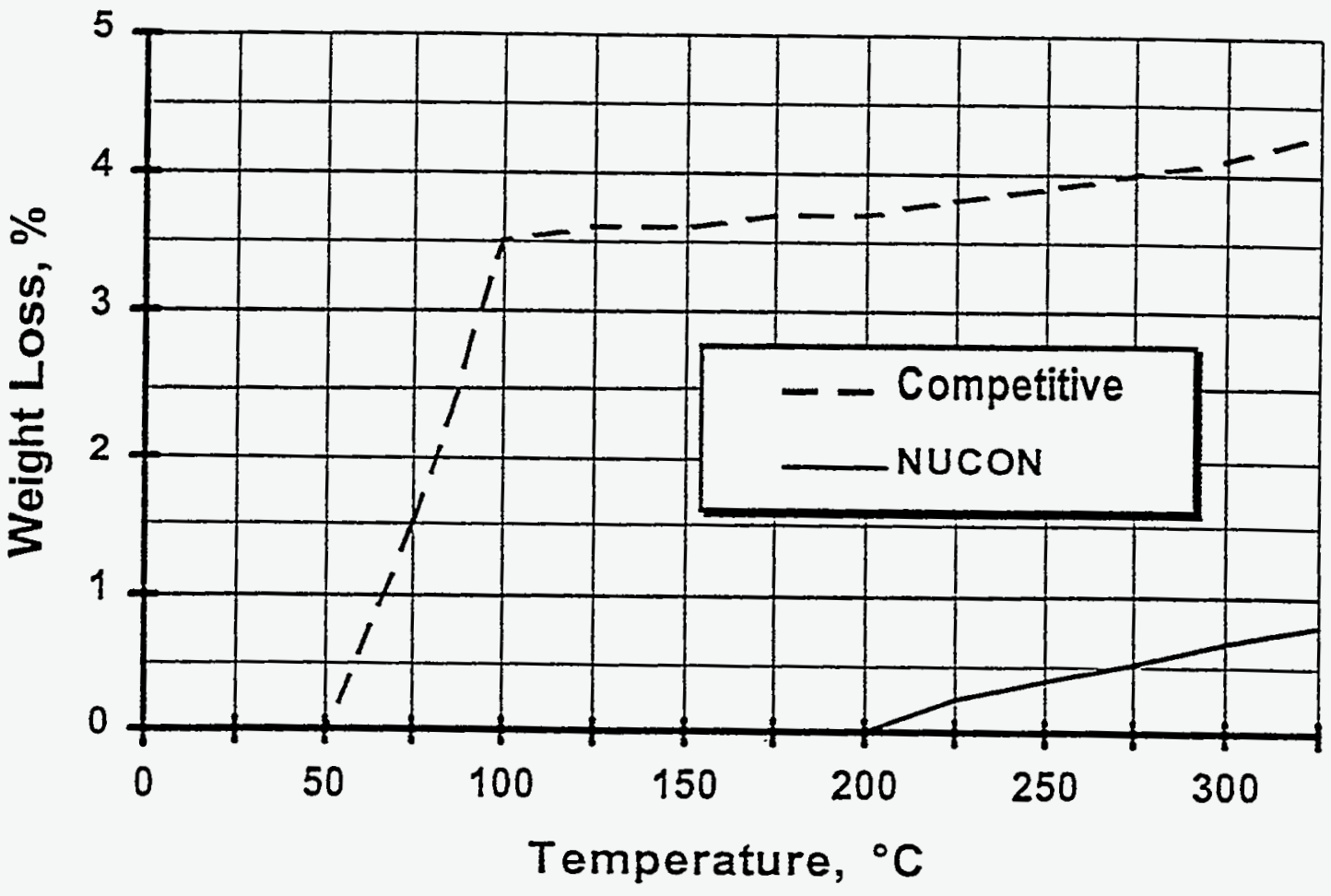

Figure 7 - Weight loss of mercury adsorbents in inert atmosphere. 
e differences are even more striking for tests conducted in air (See Figure 8). The air nosphere seems to stabilize the sulfur in the competitive product at low temperatures. However, ? weight loss at temperatures above $275^{\circ} \mathrm{C}$ indicates that both the sulfur impregnant and some the carbon is being oxidized. For the NUCON material, only a portion of the sulfur is lost at at temperature.

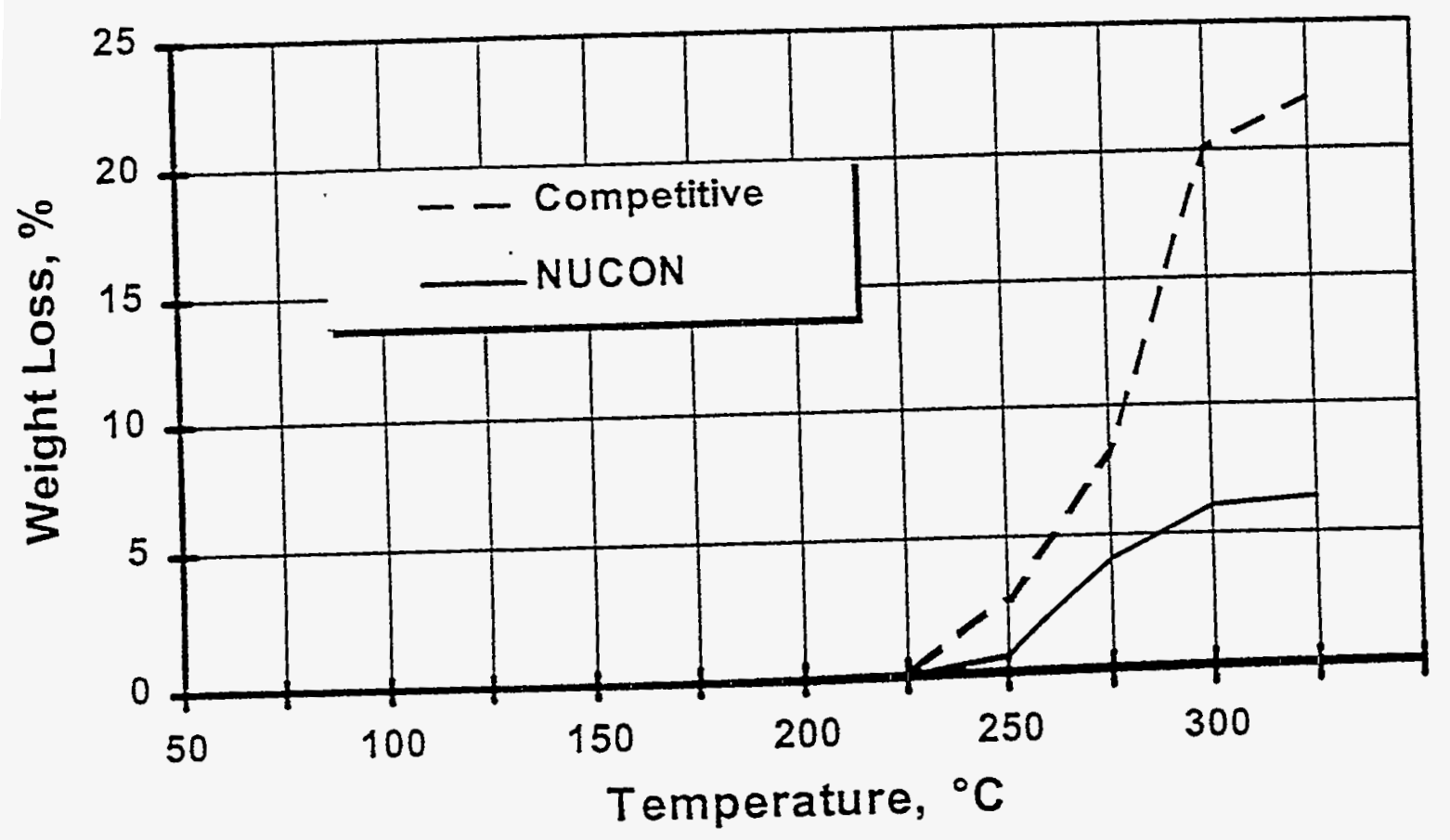

Figure 8 - Weight loss of mercury adsorbents in air. 


\section{LIQUID PHASE APPLICATIONS}

NUCON also produces mercury removal adsorbents for liquid phase applications. The MERSORB ${ }^{\otimes} L$ designation is used for products designed to remove mercury from the liquid phase.

There are two products used for liquid phase application:

- $\quad$ MERSORB ${ }^{\circledR}$ LW for liquid phase, aqueous solutions

- MERSORB ${ }^{\otimes}$ LH for liquid phase hydrocarbons

The impregnant used to make LW grade is insoluble in water. The impregnant used to make LH grade is insoluble in common hydrocarbons. If a mercury adsorbent is required for organic compounds other than hydrocarbons, contact the NUCON Chemistry Department.

Both the LW and the LH grades are supplied as 3 and $1.5 \mathrm{~mm}$ diameter pellets.

\section{Mercury Removal from Water}

The MERSORB ${ }^{\circledR}$ LW grades chemically react with elemental mercury or water-soluble mercury saits within the pore structure of the adsorbent. Since the solubility of elemental mercury in water is below hazardous levels (.064 mg/liter) it is rarely a problem. However, soluble mercury salts can be present at much higher concentrations in various contaminated streams and they may need to be treated.

The mercury adsorption capaciry of MERSORB ${ }^{\circledR} L W$ is concentration dependent. Typical design contact times are in the range of 20-40 minutes at ambient temperature. The MERSORB ${ }^{\otimes}$ LW grades can be used at temperatures up to $90^{\circ} \mathrm{C}$.

If large amounts of dissolved organic material are also present in the aqueous streams, an unimpregnated carbon (NUSORB GC60-3) should be used as a guard bed to increase the life and efficiency of the MERSORB ${ }^{\circledR}$ LW for mercury removal.

\section{Adsorption Capacity}

Figure 9 shows an isotherm for adsorption of mercury from water. Water (pH 7) containing 50 ppm mercury (as $\mathrm{Hg}^{+2}$ from $\mathrm{HgCl}_{2}$ ) was contacted with various amounts of MERSORB ${ }^{\otimes} \mathrm{LW}-3$ ground to -325 mesh. After 24 hours, the carbon was filtered out and the residual mercury concentration in the filtrate was determined by Atomic Absorption Spectroscopy. 


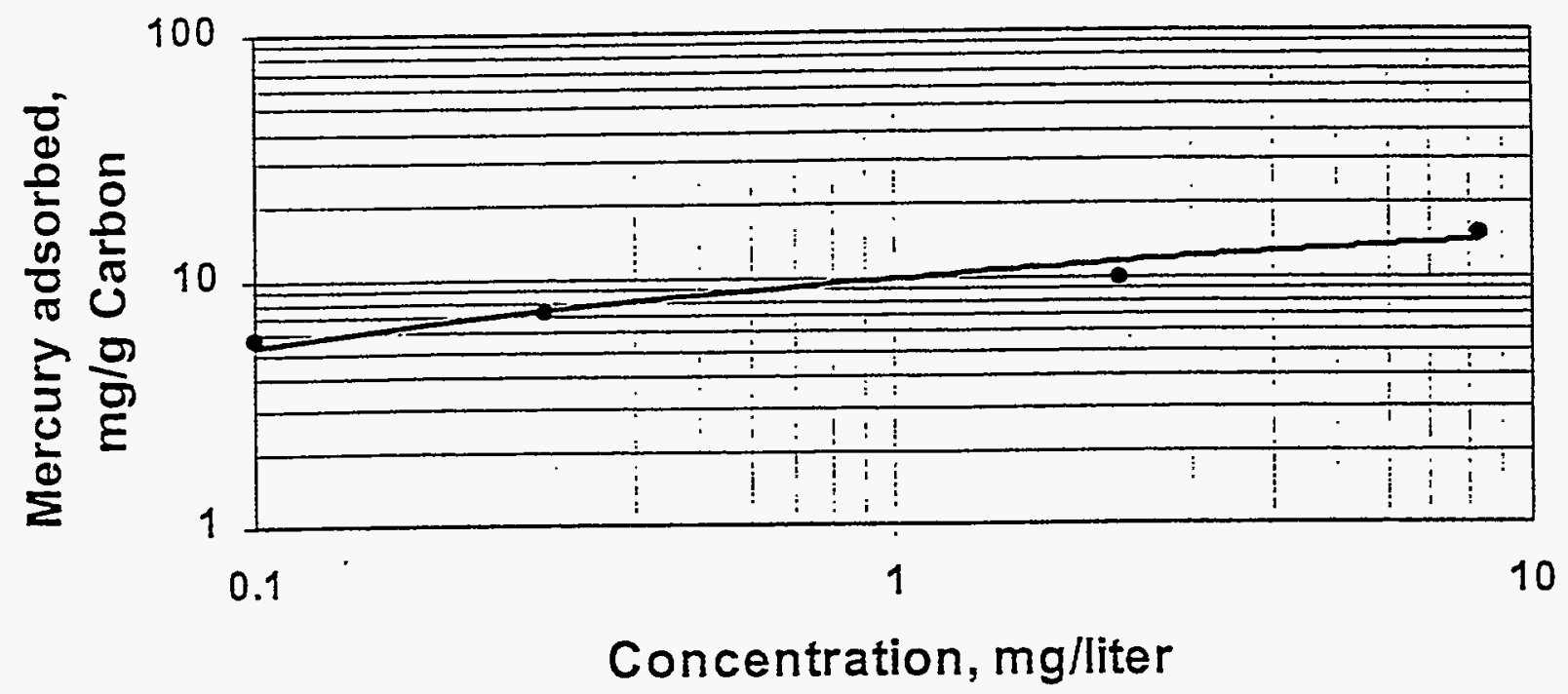

Figure 9 - Adsorption of mercury from water by MERSORB® LW.

\section{Effect of Acidity}

The $\mathrm{pH}$ of the water influences the adsorption capacity for mercury. MERSORB ${ }^{\circledR} \mathrm{LW}$ was ground to $-325 \mathrm{mesh}$ and $0.1 \mathrm{~g}$ was mixed with $100 \mathrm{ml}$ of water containing $97.4 \mathrm{mg}$ mercury/liter water. The $\mathrm{pH}$ was adjusted with $\mathrm{NaOH}$ solution. The residual mercury concentration was measured after 24 hours. Those results are shown in Table 6.

Table 6. Effect of pH on Mercury Removal

$\begin{array}{lllll}\mathrm{pH} & 3.2 & 7 & 8 & 10 \\ \text { Amount removed, \% } & 44 & 80 & 95 & 99\end{array}$

Less than half of the mercury was removed at $\mathrm{pH} 3.2$ while over $99 \%$ was removed at $\mathrm{pH} 10$. 


\section{Effect of Residence Time}

While most of the adsorption occurs in less than one day, diffusion and reaction of mercury into the adsorbent exposes unreacted impregnant which further reduces the mercury concentration in solution. The impact of both time and $\mathrm{pH}$ are shown in Figure 10.

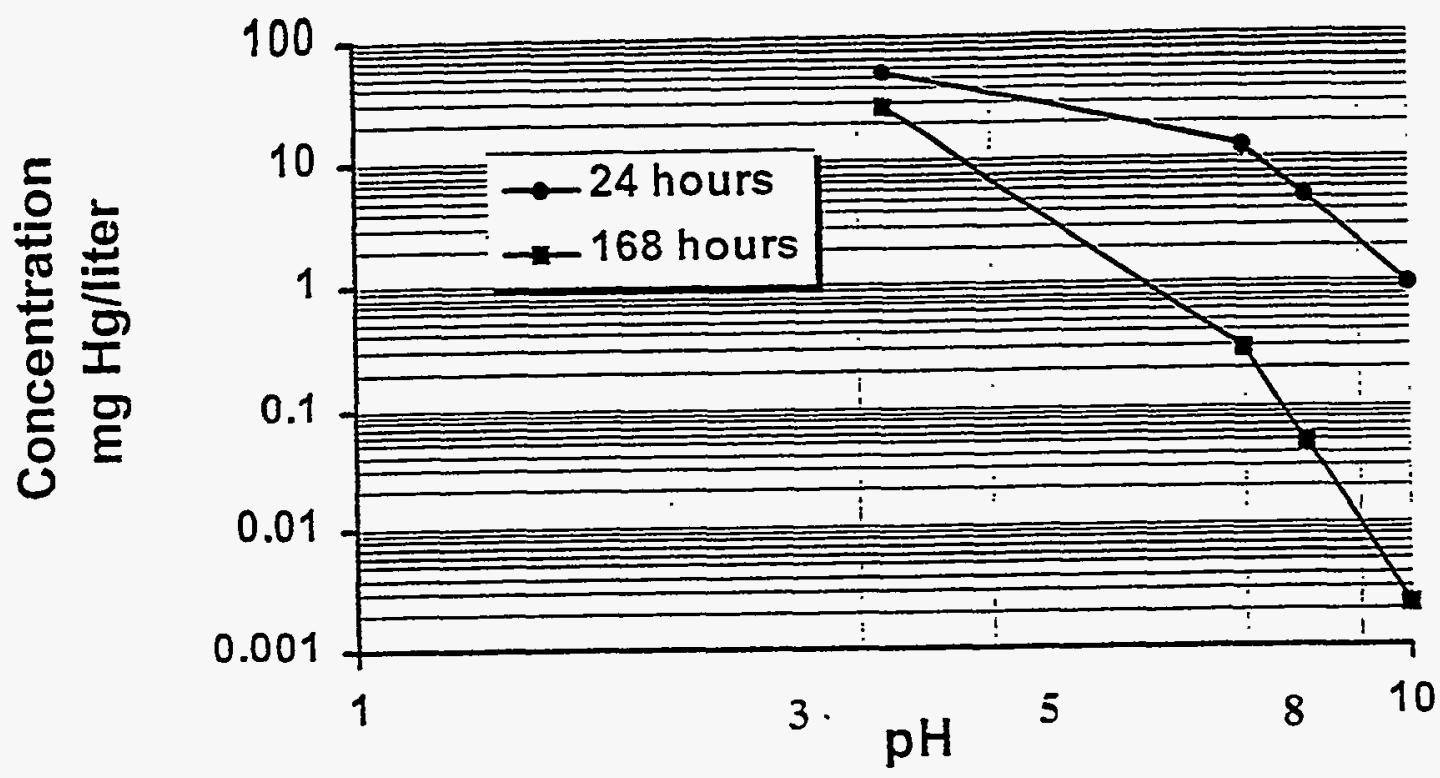

Figure 10 - Effect of $\mathrm{pH}$ and time on mercury removal from water.

If processing time is not a constraint, very low residual levels of mercury can be obtained by extending the contact time.

\section{Effect of Carbon Substrate}

The importance of choosing the optimum type of activated carbon for use as the base material for impregnation has been mentioned. Diffusion is the rate limiting step, especially in liquid phase applications. The macropore sructure of the carbon plays an important role in improving the rate of adsorption. Tests were performed using the type of carbon impregnated by the NUCON technique and two competitive materials recommended for mercury removal from water. The samples were ground to powder form to eliminate sizing variables. Portions of carbon were mixed with water containing $100 \mathrm{mg}$ mercury $\left(\mathrm{as}^{\mathrm{H}} \mathrm{Hg}^{+2}\right.$ ) per liter of water. The residual concentration of mercury was measured at various time intervals. The results are shown in Figure 11. 
NUCON $11 B 28$ R1 - 10/97

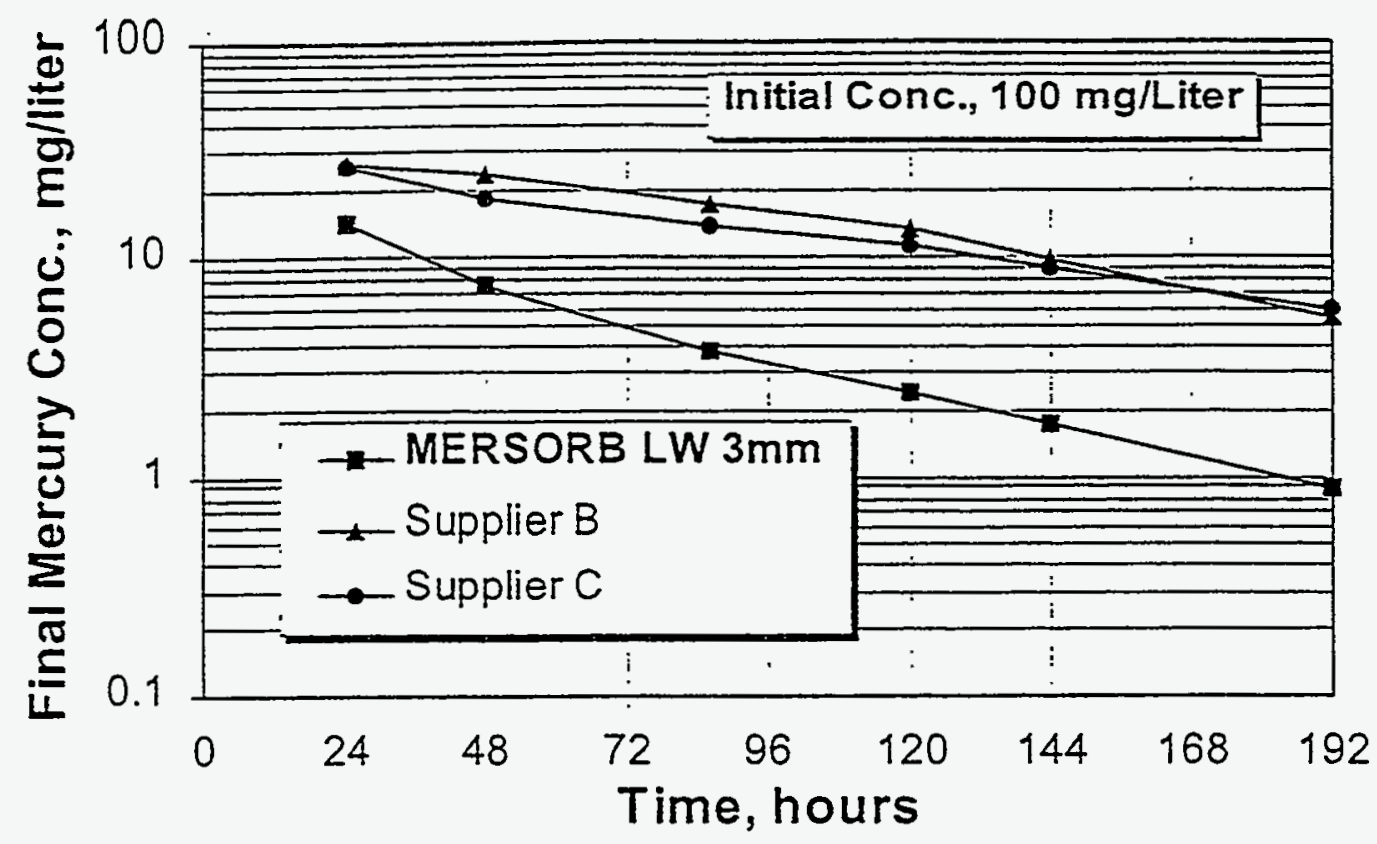

Figure 11 - Adsorption of mercury vs. time.

The $3 \mathrm{~mm}$ substrate provided a much lower residual mercury level than the competitive products.

\section{Oak Ridge National Laboratory Studies}

Mixed wastes containing mercury must be treated at a number of nuclear facilities. As a part of a program to obtain preliminary technical data, a team at Oak Ridge National Laboratory performed lab studies using a solution of mercury in water synthesized to duplicate some of the actual wastes. ${ }^{(6)}$ They found that MERSORB ${ }^{\otimes}$ LW was effective in this application. By varying solution conditions, they found that mercury uptake was slightly slower at low $\mathrm{pH}$ and that competing cations reduced the total amount of mercury removed. While theoretical mercury capacity is $0.71 \mathrm{~g} / \mathrm{g}$ of MERSORB ${ }^{\circledR} \mathrm{LW}$, at the low concentrations used for the tests, the capacity was $0.12 \mathrm{~g} / \mathrm{g}$ at neutral $\mathrm{pH}$. The rate of mercury adsorption was found to follow first-order kinetic behavior. The length of the mass transfer zone under the specific test conditions used was estimated to be 6.2 centimeters. 
In another study, MERSORB ${ }^{\circledR}$ LW was evaluated for its mercury removal capability from water streams which contain dissolved saits. ${ }^{\text {(n) }}$ In these experiments, the weight Distribution Coefficient (D), that is, the adsorbed amount per kilogram of dry adsorbent divided by the amount per liter solution, was determined at two mercury concentrations from a $0.05 \mathrm{M}$ sodium nitrate and a $0.05 \mathrm{M}$ sodium chloride solution. The mercury in the influent was present as $\mathrm{Hg}^{+2}$.

\section{Table 4. Distribution Coefficient of $\mathrm{Hg}^{+2}$ on MERSORB ${ }^{\otimes} \mathrm{LW}$ $\mathrm{Hg}^{+2}$ Salt Concentration $\quad$ Trace $0.001 \mathrm{~mol} \mathrm{Hg} / \mathrm{kg}$ \\ From $0.05 \mathrm{M} \mathrm{NaN03}$ \\ From $0.05 \mathrm{M} \mathrm{NaCl}$

$16,500(1 / \mathrm{kg})$
$1,000,000(1 / \mathrm{kg})$ \\ $76,200(1 / \mathrm{kg})$ \\ $175,000(1 / \mathrm{kg})$}

\section{Mercury Removal from Hydrocarbon Liquids}

Tests have been performed in the NUCON laboratory using MERSORB ${ }^{\circledR}$ grade LH to remove mercury from heptane. Equilibrium adsorption results are shown in Figure 9.

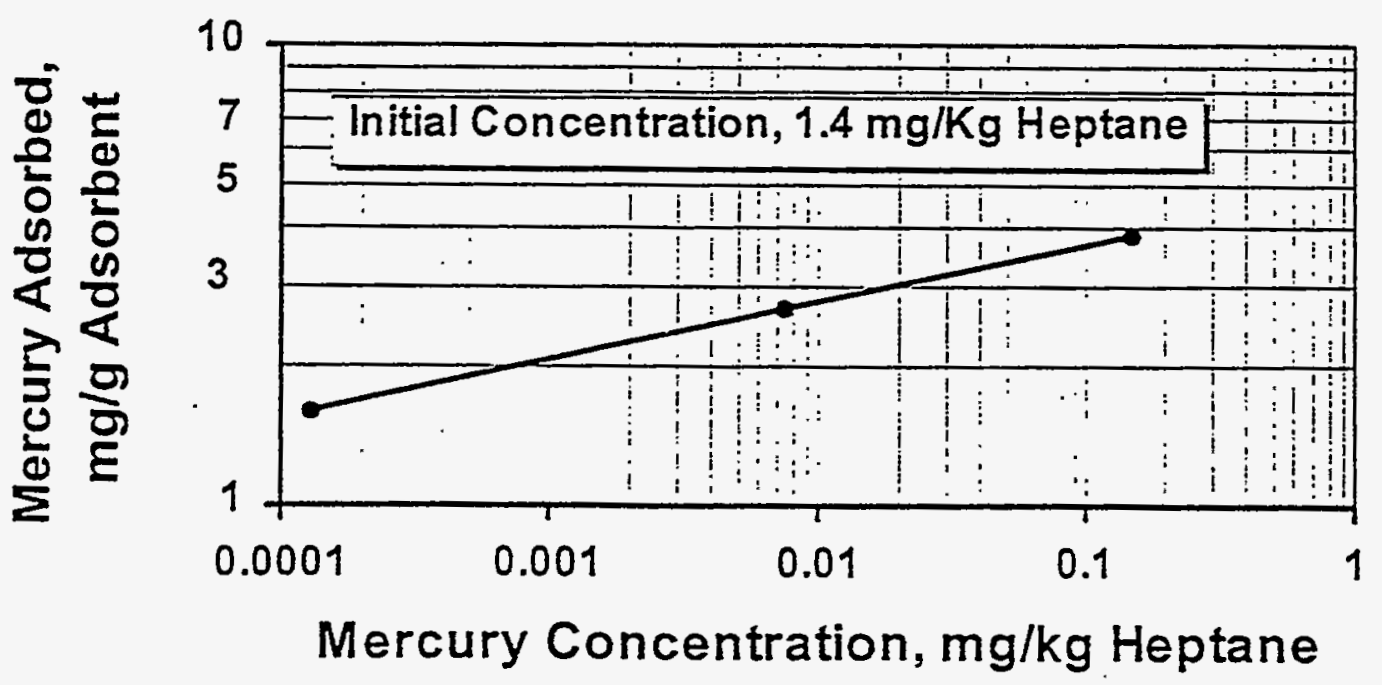

Figure 12 - Mercury adsorption from heptane, MERSORB® LH. 
NUCON 11B28 R1 - 10/97

\section{CASE HISTORIES}

\section{Water from Air Scrubbers}

For some small medical waste incinerators, the exhaust gas is passed through a water scrubber to remove particulates and water soluble compounds. If mercury is present in the exhaust, it will be contained in the scrubber water. This scrubber water, containing 0.3 ppmw mercury, is passed through a column of MERSORB ${ }^{\otimes}$ LW to remove the mercury. Effluent levels of less than 0.2 ppbw $\mathrm{Hg}$ are being obtained.

\section{Mercury Cell Hydrogen}

High purity hydrogen chloride is manufactured by reacting hydrogen and chlorine. One location uses hydrogen from mercury cells and the mercury must be removed to meet the specifications for the $\mathrm{HCl}$. Mercury concentrations up to $300 \mathrm{ppb}$ were reduced to less than $0.01 \mathrm{ppb}$ in a single column of MERSORB ${ }^{\otimes} 3 \mathrm{~mm}$ diameter pellets. This system has been in operation for over eight years with $100 \%$ mercury removal.

\section{Natural Gas Processing}

A large liquified natural gas (LNG) plant in Northern Algeria was processing natural gas with high mercury contents in aluminum heat exchangers. In order to protect the equipment from corrosion, large beds of MERSORB ${ }^{\otimes} 4 \mathrm{~mm}$ pellets were installed. There are also several installations of MERSORB ${ }^{\circledR}$ adsorbent in U.S. LNG plants.

\section{Mine Atmosphere}

A gold mining plant in Nevada encountered concentrations well above the TLV in the enclosed processing area. An air purification system containing MERSORB ${ }^{\otimes} \mathrm{mm}$ pellets was installed. The mercury level has been reduced to below the TLV. 


\section{Mercury Waste Remediation}

Waste materials containing mercury are retorted to make them nonbazardous. The effluent gas is cooled to recover liquid mercury but the temperature must remain above the water boiling point to prevent large quantities of condensate. The high temperature capability of the MERSORB ${ }^{\circledR}$ adsorbent provides good efficiency at temperatures of $250^{\circ} \mathrm{F}$. The pelleted adsorbent provides good flow distribution.

\section{Mercury Removal from Waste Water}

Water containing up to $100 \mathrm{ppmw}$ mercury is processed in a system which includes pretreatment to reduce the mercury levels to around 1 ppmw. Three vessels in series, containing MERSORB ${ }^{\circledR}$ IW mercury adsorbent, reduce the concentration to below detectable levels $(<20 \mathrm{ppbw})$. The water can then be disposed of as nonhazardous waste. Empty bed contact times (EBCT) range from 20-30 minutes at a feed water $\mathrm{pH}$ of 7.0.

\section{Fluorescent Lamp Recycling}

A major manufacturer of fluorescent lamp recycling machines uses MERSORB ${ }^{\circledR} \mathrm{Hg}$ adsorbents in both its original and new model machines. The treated air is within regulatory emission control limits. 
NUCON 11B28 R1 - 10/97

\begin{abstract}
the OPERATING GUIDELINES
1. Do not use these products for acidic solutions. Acids reacting with sulfur compounds can generate hydrogen sulfide which is poisonous. Removal efficiency decreases at a $\mathrm{pH}$ below 7 .

2. When non-mercury impurities must also be removed, it may be desirable to use "guard" adsorbent beds in service before the MERSORB ${ }^{\circledR}$ beds to remove these impurities and increase the life of the mercury adsorption bed.

3. The mercury removed by the sulfur impregnated MERSORB ${ }^{\circledR}$ materials is converted by the adsorbent to mercuric sulfide, a naturally occurring compound. Spent adsorbent should be handled according to appropriate disposal procedures and according to applicable safety and transportation regulations.
\end{abstract}

4. For optimum removal efficiency, it is always preferable to operate a deep bed at high velocity rather than shallow adsorbent bed at a low velocity.

5. It is common for natural gas streams to be saturated with water. Since high relative humidity interferes with mercury adsorption, it is important to raise the temperature of the gas enough to reduce the relative humidity to at least $70 \%$. This will also eliminate the possibility of getting liquid water on the adsorbent beds. It is also helpful to heat trace the piping between the heater and the adsorber to prevent cooling and condensation.

For sulfur impregnated activated carbons, the inlet gas temperature should be limited to $65^{\circ} \mathrm{C}$ to eliminate vaporization of the sulfur. Entrained hydrocarbon liquids should also be removed to avoid leaching of the sulfur impregnant.

7. When the adsorbent is placed into service, there is significant heat evolved from the initial adsorption of hydrocarbon vapor or liquid. The gas flow to the vessel should be slowly increased from a low initial level while the gas temperature is carefully monitored. The temperature should be held below $150^{\circ} \mathrm{C}$. This start-up procedure may take as long as four hours before an equilibrium level is achieved. Once this occurs, full flow rate can be applied.

8. It is important to have effective liquid knockout ahead of gas phase mercury adsorption beds. Liquid hydrocarbons can dissolve the sulfur impregnant. Any liquids entering or condensing in the adsorbent bed interfere with the mercury adsorption rate and capacity. 


\section{TECHNICAL SUPPORT}

NUCON technical personnel are available to assist in the design of mercury removal systerns.

A. Basic adsorption equilibrium data can be determined by laboratory tests.

B. Bench scale apparatus is used to provide dynamic adsorption data including shape, size and movement of the mass transfer zone. The process design can be developed from this information.

C. Engineering of the mercury removal process can be performed by NUCON. If desired, NUCON can provide the complete installation.

D. On-site technical services and testing are available.

NUCON can advise users concerning potential recovery of mercury from spent adsorbent beds. 


\section{REFERENCES}

1. Lewis, Larry. "Measurement of Mercury in Natural Gas Streams," presented at the 74th Annual GPA Convention, San Antonio, Texas. March 1995.

2. Documentation of the Threshold Limit Values and Biological Exposure Indices, Sixth Edition, 1991, ACGIH, p 881.

3. Schroeder, William H. "Sampling and Analysis of Mercury and its Compounds in the Atmosphere," Environmental Science \& Technology, Vol. 16, No. 7, 1982. p 396A.

4. Ibid. p 394A.

5. Spencer, James N. and Voight, Adolf F., "Thermodynamics of the Solution of Mercury," Journal of Physical Chemistry, Vol. 72, No. 2, Feb. 1968. p 466.

6. Klasson, K. T. et al, "Mercury Removal from Liquid and Solid Mixed Wastes, Proceedings," WM95, Feb. 26 - Mar 2, 1995, Tuscon AZ.

7. Poutsma, M.L., Ferris, L.M., Keller, O.L., Mesmer, R.E., Chemistrv Division Annual Progress Report. 1985, ORNL-615D. p 182-184. 